\title{
Communicating Science
}

\section{Through Visualization}

\section{In an Age of Alternative Facts}

\author{
Kalina Borkiewicz, AJ Christensen, and John E. Stone \\ University of Illinois at Urbana-Champaign
}

Communicating Science Through Visualization in an Age of Alternative Facts

Cinematic scientific visualization demystifies complex scientific concepts for general audiences, which helps them defend themselves against misinformation in popular media.

Researchers from the University of Illinois will show techniques for representing data accurately while making it not only approachable, but beautiful and compelling to experts and non-experts alike.

\footnotetext{
Permission to make digital or hard copies of part or all of this work for personal or classroom use is granted without fee provided that copies are not made or distributed for profit or commercial

advantage and that copies bear this notice and the full citation on the first page. Copyrights for third-party components of this work must be honored. For all other uses, contact the

Owner/Author.

Copyright is held by the owner/author(s).

SIGGRAPH'17 Courses, July 30 - August 03, 2017, Los Angeles, CA, USA

ACM 978-1-4503-5014-3/17/07.

http://dx.doi.org/10.1145/3084873.3084935
} 


\section{Who Are We}

"Abstracting" AJ

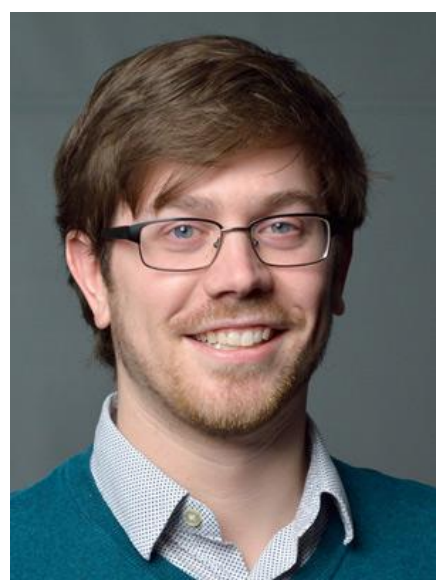

"Interpolating" Kalina

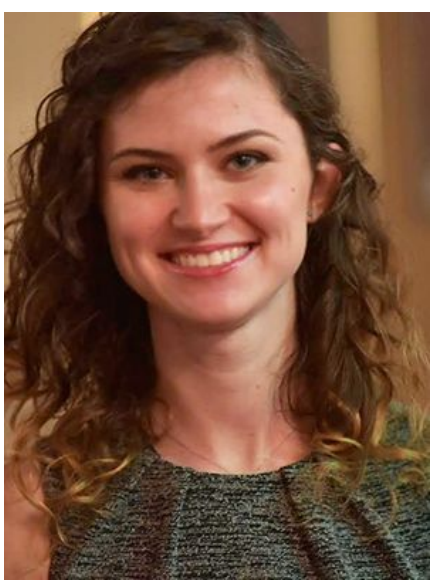

"Extrapolating" John

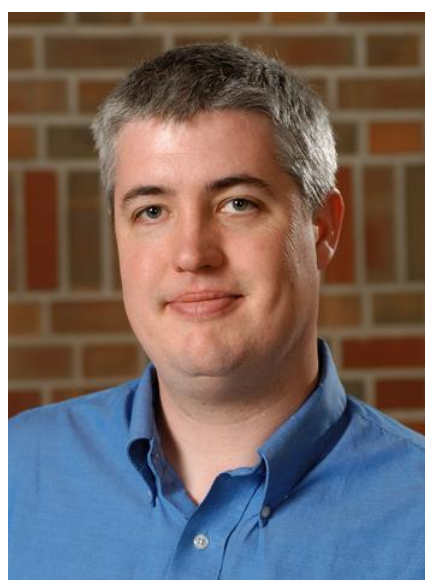

AJ Christensen

Visualization Programmer

Advanced Visualization Lab

National Center for Supercomputing Applications

University of Illinois at Urbana-Champaign

Kalina Borkiewicz

Visualization Programmer

Advanced Visualization Lab

National Center for Supercomputing Applications

University of Illinois at Urbana-Champaign

\section{John Stone}

Senior Research Programmer

Theoretical and Computational Biophysics Group

Beckman Institute for Advanced Science and Technology

University of Illinois at Urbana-Champaign 


\section{Table of Contents}

1) Overview ... 4

a) Definitions ... 5-9

b) Recent Examples ... 12

2) Cinematic Scientific Visualization ... 19

3) Communicating Complex Scientific Concepts with Imagery ... 25

a) tools ... 28

i) partiview ... 29

ii) virtual director ... 34

iii) houdini ... 39

iv) $\mathrm{yt} \ldots 46$

v) ytini ...55

vi) VMD ...58

b) techniques ... 117

i) abstraction and representation ... 118

ii) isolation and simplification ... 125

iii) interactive exploration ... 128

iv) camera design ... 131

v) interpolation ... 139

vi) registration ... 154

vii) compositing ... 163

4) Validation and Interpretation ... 178

a) visualization can be misleading ... 179

b) validation ... 186

c) evaluation of learning effectiveness ... 191

d) re-use for maximum impact ... 197

5) Thanks ... 201 
Overview 


\section{Definitions}

\section{Renaissance Team $n$.}

1. A cross-disciplinary group of experts in science, technology, and art, who work together to build extremely effective science outreach projects.

The Advanced Visualization Lab (AVL) is a research team at the National Center for Supercomputing Applications (NCSA) at the University of Illinois at Urbana-Champaign. The AVL is led by Professor Donna Cox, who coined the term "Renaissance Team", with the belief that bringing together specialists of diverse backgrounds creates a team that is greater than the sum of its parts, and cultivates innovation and creative group problem-solving. The AVL specializes in creating high-quality cinematic scientific visualizations of supercomputer simulations for public outreach. The AVL has contributed to dozens of television, digital fulldome, and IMAX science documentaries. 


\section{Definitions}

Scientific simulation $n$.

1. A scientific simulation is a computational model that solves physical and statistical equations to simulate real-world phenomena. These simulations are often done on supercomputers.

As a part of the National Center for Supercomputing Applications, the AVL works with supercomputer data from scientific simulations. These simulations allow scientists to run virtual experiments where a physical experiment is either too costly, time-consuming, or impossible. A scientific simulation differs from the Hollywood definition of a "FX simulation" for animation, which can be done in a graphics engine. In this course, when we use the word "simulation", we are referring to a scientific simulation. 


\section{Definitions}

Alternative Facts $n$.

1. Statements which appear to be true only when taken out of context.

Science $n$.

1. The process of methodically reducing uncertainty about the answer to a question.

Today a large portion of society is not taking scientists or fact-based journalism seriously. Sharing real science in a noisy media landscape is even harder when you consider that what science is by definition is uncertain. It is an uphill battle to convince the public that scientific findings are less uncertain than a person's opinions or anecdotal experience, especially when science-deniers seem certain of their claims.

Further complicating the issue is a misunderstanding of scientists, who are viewed by some as intellectual elitists who don't care what effects their discoveries have on the economy and blue collar laborers. Science is not always trusted because scientists can change their minds and the scientific method is not well understood by the general population.

The remedy to this miscommunication is many-sided, but cinematic scientific visualization is uniquely positioned to help better inform the public in a place they are comfortable and feel capable of participating in the discussion. 


\section{Definitions}

Cinematic Scientific Visualization $n$.

1. Production-quality, data-driven imagery created with movie-making tools with good composition, camera direction, and artistic aesthetics suitable for distribution in immersive giant screen theaters.

Scientific Visualization $n$.

1. Imagery created using data with spatial 3D coordinates, often calculated on large computing clusters.
Scientific Illustration $n$

1. Imagery created based on expert input but using predominantly artistic tools.
Information Visualization $n$

1. Imagery created using relational data which often has no direct mapping to spatial coordinates.

We have used the phrase "cinematic scientific visualization" a few times already, so let us explicitly define it, because it differs from some related terms. 


\section{Definitions}

\section{Cinematic Scientific Visualization $n$.}

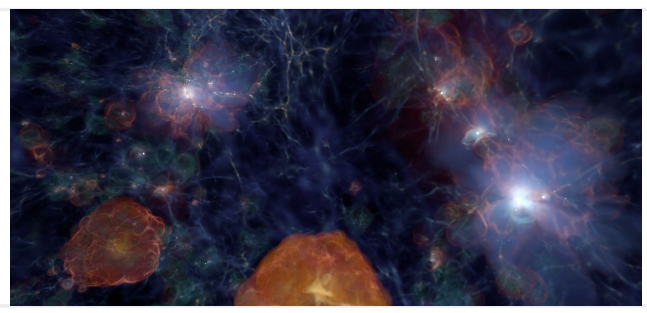

Scientific Visualization $n$.

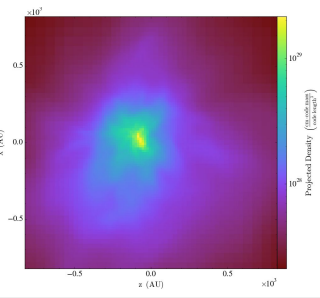

Scientific Illustration $n$.

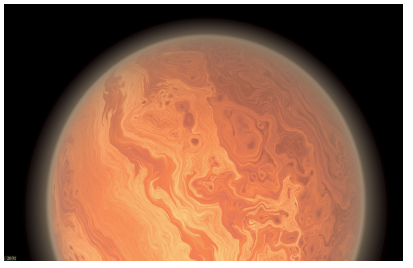

Information Visualization

Data About Things

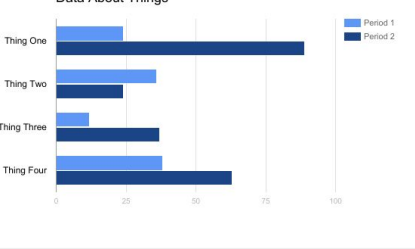

"Visualization" is a means of creating imagery from collected or calculated data, which juxtaposes it with "illustration", which is a means of creating imagery based on advice and intuition. "Scientific visualization" is an industry term used to categorize visualizations done on spatial data which often translates naturally to a 3-dimensional virtual environment. The other primary category of visualization is "information visualization" which is used for relational or other non-spatial data, and is more frequently seen in 2-dimensional representations like graphs, charts, and networks. 


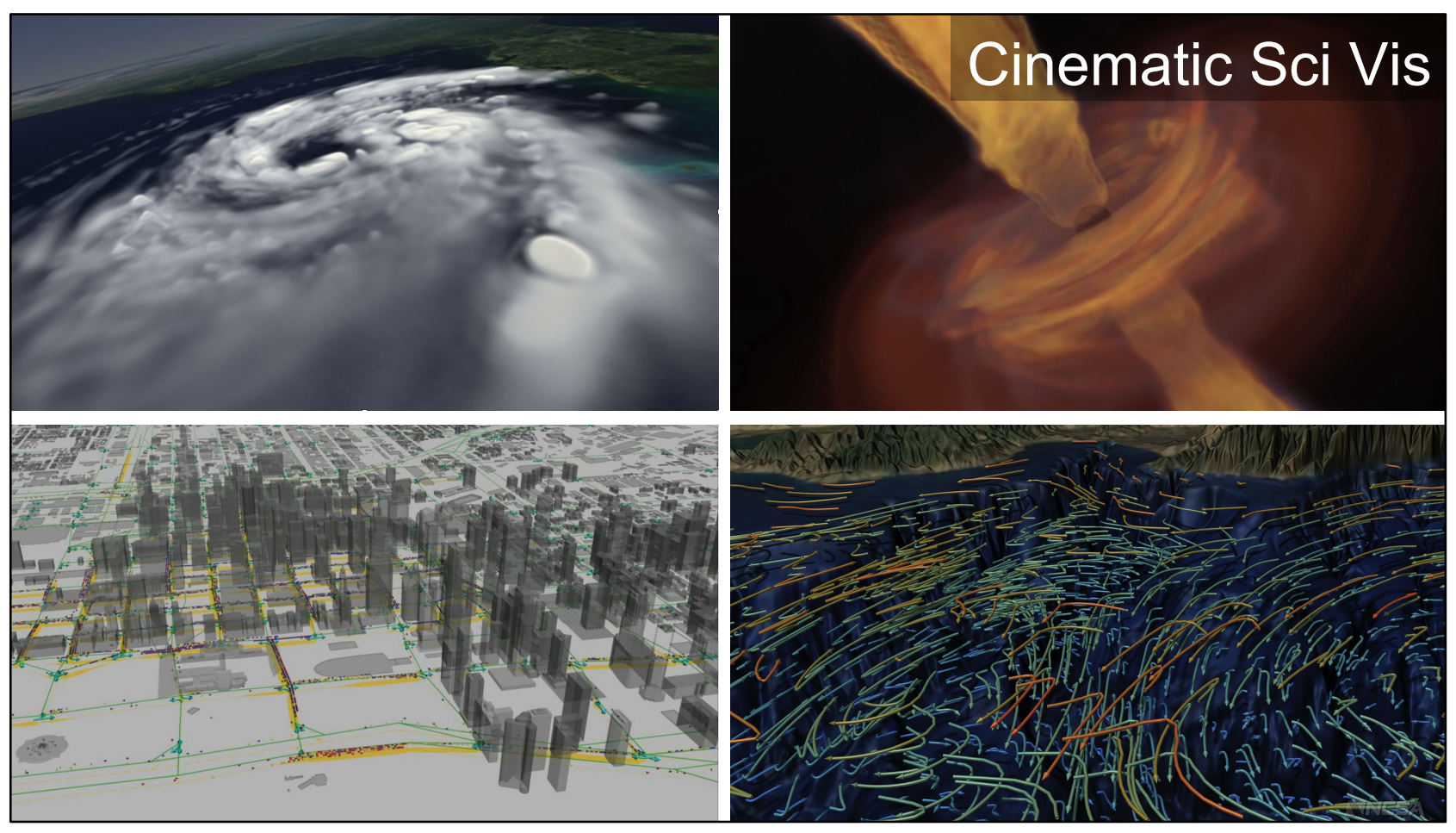

Cinematic scientific visualization is an especially accessible form of science communication, because it exploits the processing power of the visual cortex in the human brain, and because it leverages a powerful familiar visual language established by Hollywood films. 


\begin{tabular}{|c|c|c|c|}
\hline $\begin{array}{c}\text { Hubble 3D } \\
\qquad(2010) \\
\text { Narrated by } \\
\text { Leonardo DiCaprio }\end{array}$ & $\begin{array}{l}\text { The Tree of Life } \\
\text { (2011) } \\
\text { Written and Directed by } \\
\text { Terrence Malick }\end{array}$ & $\begin{array}{c}\text { Dynamic Earth } \\
\qquad(2012) \\
\text { Narrated by Liam Neeson }\end{array}$ & $\begin{array}{l}\text { Destination } \\
\text { Solar System } \\
\qquad(2014) \\
\text { At Chicago's } \\
\text { Adler Planetarium }\end{array}$ \\
\hline $\begin{array}{c}\text { Solar Superstorms } \\
\qquad(2015) \\
\text { Narrated by } \\
\text { Benedict Cumberbatch }\end{array}$ & $\begin{array}{l}\text { SuperTornado: } \\
\text { Anatomy of a } \\
\text { MegaDisaster } \\
(2016)\end{array}$ & $\begin{array}{c}\text { A Beautiful Planet } \\
(2016) \\
\begin{array}{c}\text { Narrated by Jennifer } \\
\text { Lawrence }\end{array}\end{array}$ & $\begin{array}{l}\text { Seeing the } \\
\text { Beginning of Time } \\
(2017)\end{array}$ \\
\hline
\end{tabular}

Recent examples of cinematic scientific visualization done by the AVL include:

IMAX Films:

Hubble 3D (2010), narrated by Leonardo DiCaprio

A Beautiful Planet (2016), narrated by Jennifer Lawrence

Narrative Films:

The Tree of Life (2011), written and directed by Terrence Malick

Europa Report (2013), directed by Sebastián Cordero

Fulldome Shows:

Dynamic Earth (2012), narrated by Liam Neeson

Destination Solar System (2014), at the Chicago Adler Planetarium

Solar Superstorms (2015), narrated by Benedict Cumberbatch

TV Documentaries:

Solar Superstorms: Journey to the Center of the Sun (2015)

SuperTornado: Anatomy of a MegaDisaster (2016)

Seeing the Beginning of TIme (2017) 


\section{Recent Examples}

Here we share describe datasets recently visualized by the AVL from the fulldome show "Solar Superstorms". 


\section{SUN SURFACE}

MATTHIAS REMPEL, M.C.M. CHEUNG

\section{SIMULATION STATS}

SPATIAL SCALE TIME SCALE RESOLUTION

DATA SIZE COMPUTED ON
$150,000 \times 75,000 \mathrm{KM}$

140 HOURS

16,800 TIMESTEPS, 600 GB

1794 TIMESTEPS, 2.8TB

KRAKEN, NATIONAL INSTITUTE

FOR COMPUTATIONAL

SCIENCES



Our team works with computational data - often generated by supercomputers. This presents many challenges, including efficient data movement, storage, and processing.

This smallest data set used in the film is a computational model of solar surface convection which eventually reveals the formation of sunspots.

Image Credits:

"Solar Superstorms": A full-dome production by NCSA, University of Illinois, Thomas Lucas Productions, Spitz Creative Media

"Active Region Scale Flux Emergence on the Sun" simulation: Matthias Rempel (High Altitude Observatory, National Center for Atmospheric Research), M.C.M. Cheung (Lockheed Martin Solar and Astrophysics Lab)

"Double Coronal Mass Ejection" simulation: Yuhong Fan (High Altitude Observatory, National Center for Atmospheric Research)

SDO Data: Steele Hill (SOHO/STEREO/SDO Media Specialist, NASA Goddard Space Flight Center)

Visualization by: Advanced Visualization Lab, National Center for Supercomputing Applications, University of Illinois at Urbana-Champaign, Donna Cox, Kalina Borkiewicz, Jeff Carpenter, AJ Christensen, Stuart Levy, Robert Patterson Funded in part by: National Science Foundation Award ACl-1445176, The Centrality of Advance Digitally Enabled Science: CADENS 


\title{
DOUBLE C.M.E
}

\author{
YUHONG FAN
}

\section{SIMULATION STATS}

INSTITUTION

SPATIAL SCALE

TIME SCALE RESOLUTION

DATA SIZE COMPUTED ON
NATIONAL CENTER FOR ATMOSPHERIC RESEARCH 1 TO 6.25 X SOLAR RADIUS 100 MINUTES

$577 \mathrm{R} \times 384 \theta \times 432 \varphi$

126 FIELD LINES

1794 TIMESTEPS, 2.8TB

YELLOWSTONE, NWSC/NCAR AND

DISCOVER, NASA CENTER FOR

CLIMATE SIMULATION

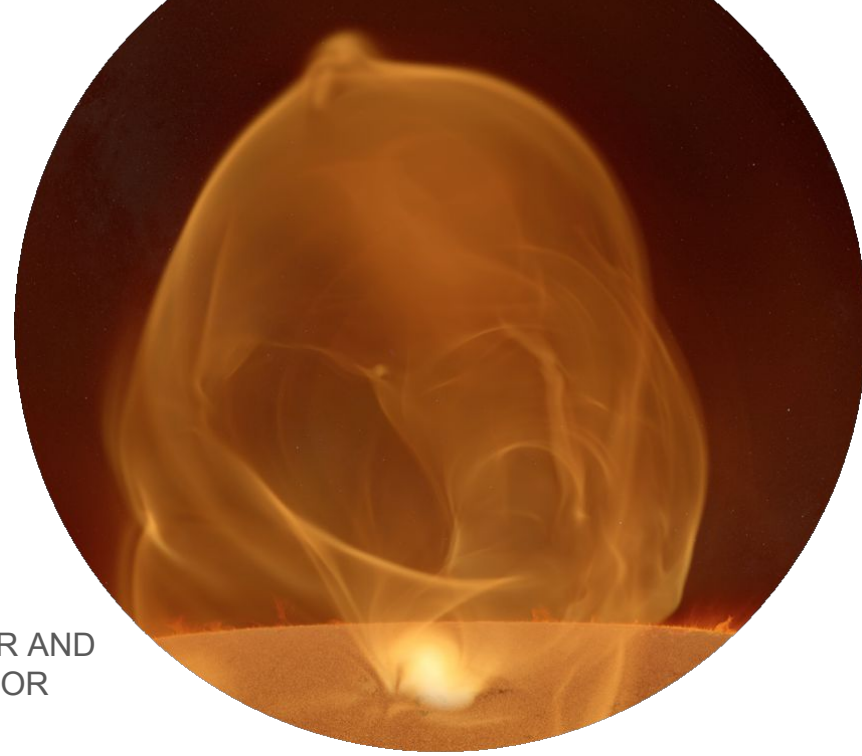

This computer model of a double coronal mass ejection event had to be re-calculated for the film because the pre-existing data was not saved out at a high enough frequency to play back smoothly when put to film.

This spherical setup is an example of volumetric data that was not calculated on uniform rectangular grid.

Image Credits:

"Solar Superstorms": A full-dome production by NCSA, University of Illinois, Thomas Lucas Productions, Spitz Creative Media

"Double Coronal Mass Ejection" simulation: Yuhong Fan (High Altitude Observatory, National Center for Atmospheric Research)

"Active Region Scale Flux Emergence on the Sun" simulation: Matthias Rempel (High Altitude Observatory, National Center for Atmospheric Research), M.C.M. Cheung (Lockheed Martin Solar and Astrophysics Lab) SDO Data: Steele Hill (SOHO/STEREO/SDO Media Specialist, NASA Goddard Space Flight Center) Visualization by: Advanced Visualization Lab, National Center for Supercomputing Applications, University of Illinois at Urbana-Champaign, Donna Cox, Kalina Borkiewicz, Jeff Carpenter, AJ Christensen, Stuart Levy, Robert Patterson Funded in part by: National Science Foundation Award ACl-1445176, The Centrality of Advance Digitally Enabled Science: CADENS 


\title{
CONVECTION
}

ROBERT STEIN, AKE NORDLUND, PATRICK MORAN

\section{SIMULATION STATS}

\author{
SPATIAL SCALE \\ TIME SCALE \\ RESOLUTION \\ $48,000^{\wedge} 2 \times 20,000 \mathrm{KM}$ \\ 12 HOURS \\ 2017 x 494 × 2017 GRID \\ DATA SIZE \\ 720 TIMESTEPS, 100 TB \\ COMPUTED ON \\ SUPERCOMPUTING DIVISION
}



This computer model of solar convection just below the surface of the sun was particularly large and slow to process. Managing memory in graphics software can get to be really difficult when working with hundreds of terabytes of time-evolving simulations.

However, often scientists do their own processing and create assets that are useful in visualization, such as the magnetic field lines seen in blue here.

Image Credits:

"Solar Superstorms": A full-dome production by NCSA, University of Illinois, Thomas Lucas Productions, Spitz Creative Media

"Magneto-Convection Emerging Flux" simulation: Robert Stein (Physics and Astronomy Department, Michigan State University), Åke Nordlund (Niels Bohr Institute, Copenhagen University, Copenhagen,Denmark, NASA grants NNX08AH44G and NNX12AH49G and NSF grants AGS 1141921 and OCl 1144506), Patrick Moran (NASA Advanced Supercomputing Division, NASA Ames Research Center)

"Active Region Scale Flux Emergence on the Sun" simulation: Matthias Rempel (High Altitude Observatory, National Center for Atmospheric Research), M.C.M. Cheung (Lockheed Martin Solar and Astrophysics Lab)

Visualization by: Advanced Visualization Lab, National Center for Supercomputing Applications, University of Illinois at Urbana-Champaign, Donna Cox, Kalina Borkiewicz, Jeff Carpenter, AJ Christensen, Stuart Levy, Robert Patterson Funded in part by: National Science Foundation Award ACl-1445176, The Centrality of Advance Digitally Enabled Science: CADENS 


\section{DYNAMO}

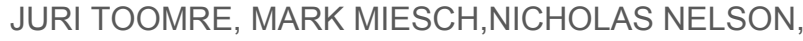
ALLAN SACHA BRUN, BENJAMIN BROWN

\section{SIMULATION STATS}

SPATIAL SCALE TIME SCALE RESOLUTION

DATA SIZE COMPUTED ON
$71 \%-98 \%$ SOLAR RADIUS 31 DAYS

$193 R \times 512 \theta \times 1024 \varphi$

1511 TIMESTEPS, 3.4 TB

LABORATORY FOR

COMPUTATIONAL DYNAMICS, UNIVERSITY OF COLORADO, BOULDER AND PLEIADES, NASA ADVANCED SUPERCOMPUTING DIVISION

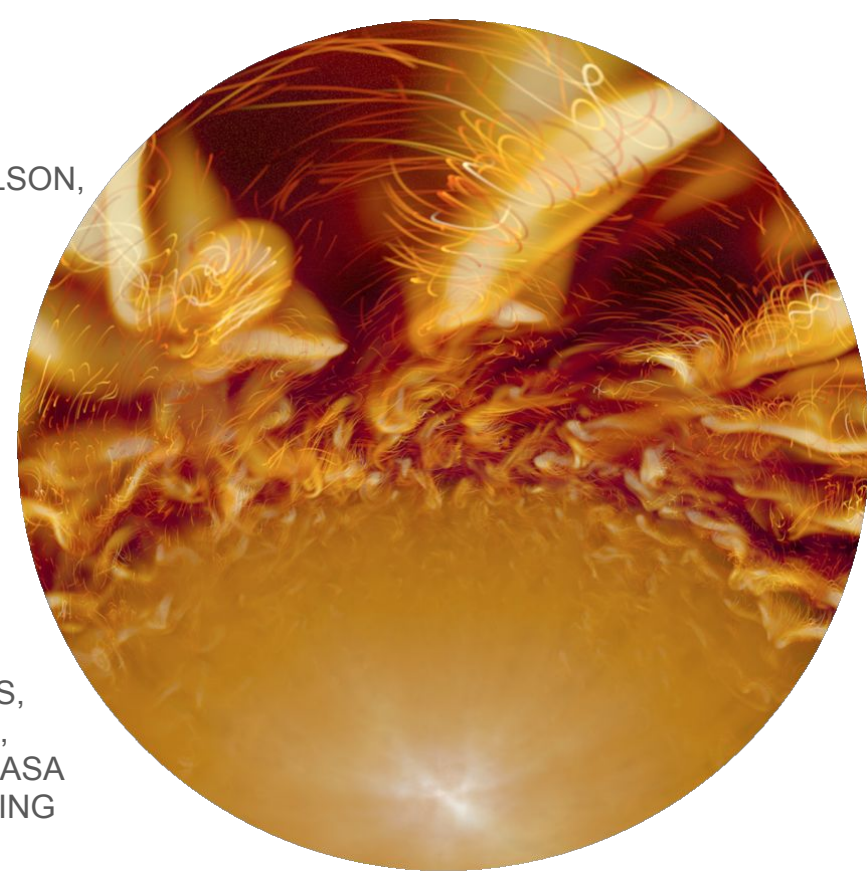

In this computational model of the 12-year solar dynamo that affects space weather, we had to work hard to identify a feature the scientists suggested we illustrated: so-called bananas of vorticity that roll around the sun's equator. This required us to process the data to create derivative data like new volume fields and streamlines.

Image Credits:

"Solar Superstorms": A full-dome production by NCSA, University of Illinois, Thomas Lucas Productions, Spitz Creative Media

"Solar Dynamo / Solar Interior" simulation by: Juri Toomre (JILA, University of Colorado Boulder), Mark Miesch (High Altitude Observatory, National Center for Atmospheric Research), Nicholas Nelson (Los Alamos National Laboratory), Allan Sacha Brun (Commissariat à l'énergie atomique et aux énergies alternatives, Saclay, France), Benjamin Brown (Laboratory for Atmospheric and Space Physics, University of Colorado Boulder)

Visualization by: Advanced Visualization Lab, National Center for Supercomputing Applications, University of Illinois at Urbana-Champaign, Donna Cox, Kalina Borkiewicz, Jeff Carpenter, AJ Christensen, Stuart Levy, Robert Patterson Funded in part by: National Science Foundation Award ACl-1445176, The Centrality of Advance Digitally Enabled Science: CADENS 


\section{PLASMA}

HOMA KARIMABADI, MAHIDHAR TATINENI, VADIM ROYTERSHTEYN, HOANH X. VU, AMIT MAJUMDAR

\section{SIMULATION STATS}

\author{
SPATIAL SCALE \\ TIME SCALE \\ RESOLUTION
}

DATA SIZE

COMPUTED ON
100 KILOMETER GRID CELLS 30 MINUTES

2D: $2048 \times 8192$ PIXELS

3D: $2048 \times 4096 \times 2048$

2D: 220 TIMESTEPS, $30 \mathrm{~GB}$

3D: 47 TIMESTEPS, 12 TB

BLUE WATERS, UNIVERSITY OF

ILLINOIS AT URBANA-CHAMPAIGN

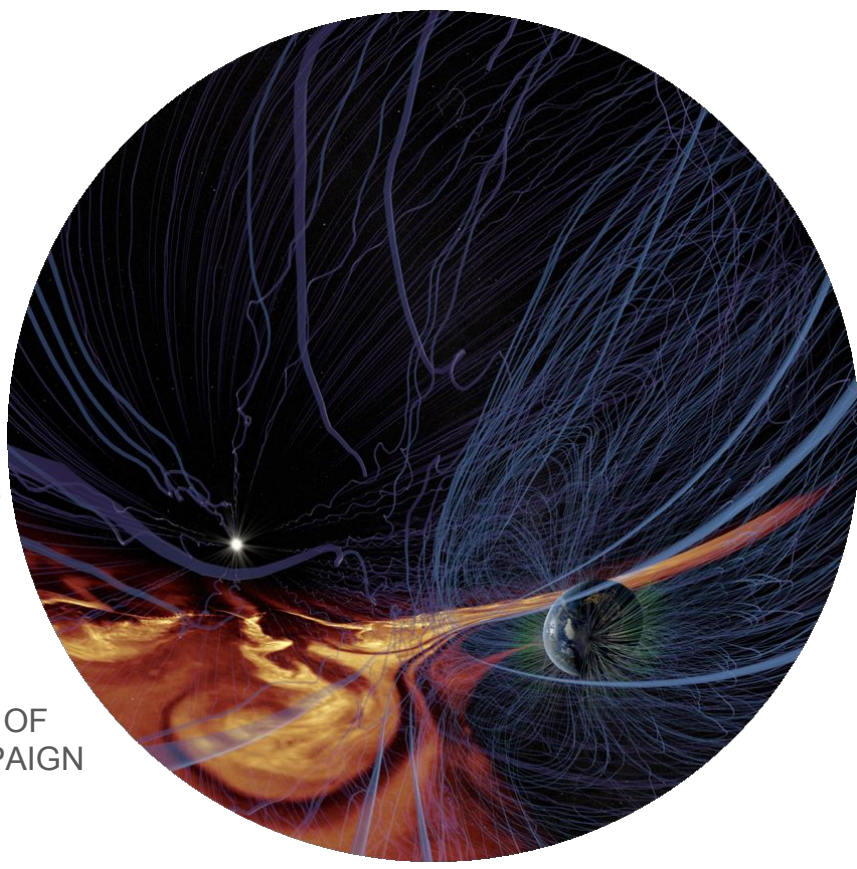

Using this computationally expensive model of a strong burst of solar wind bombarding Earth's magnetosphere, we transitioned from low-frequency 3-dimensional data to high-frequency 2-dimensional data in a single seamless camera move. Often the data is so big that we can't animate a smooth evolution and must find creative ways to present the data that is available.

Image Credits:

"Solar Superstorms": A full-dome production by NCSA, University of Illinois, Thomas Lucas Productions, Spitz Creative Media

"Solar Plasma Interacting with Earth's Magnetic Field" simulation by Homa Karimabadi (University of California San Diego, Center for Astrophysics and Space Sciences), Mahidhar Tatineni (San Diego Supercomputer Center), Vadim Roytershteyn (Space Science Institute), Hoanh X. Vu (University of California San Diego), Amit Majumdar (San Diego Supercomputer Center)

Visualization by: Advanced Visualization Lab, National Center for Supercomputing Applications, University of Illinois at Urbana-Champaign, Donna Cox, Kalina Borkiewicz, Jeff Carpenter, AJ Christensen, Stuart Levy, Robert Patterson yt visualization toolkit for volumetric data: Matt Turk, Research Scientist at the National Center for Supercomputing Applications Research, Assistant Professor in the Astronomy department, University of Illinois at Urbana-Champaign Funded in part by: National Science Foundation Award ACl-1445176, The Centrality of Advance Digitally Enabled Science: CADENS 


\title{
UNIVERSE
}

JOHN H. WISE

\section{SIMULATION STATS}

\author{
INSTITUTION \\ SPATIAL SCALE \\ TIME SCALE \\ RESOLUTION \\ DATA SIZE \\ COMPUTED ON \\ GEORGIA TECH \\ UP TO 50,000 PARSECS \\ 200 MILLION YEARS \\ AMR SPARSE GRID 256^3 \\ 12 LEVELS OF REFINEMENT \\ UP TO 330,000 PARTICLES \\ 330 TIMESTEPS, 2 TB \\ BLUE WATERS, UNIVERSITY OF \\ ILLINOIS AT URBANA-CHAMPAIGN
}

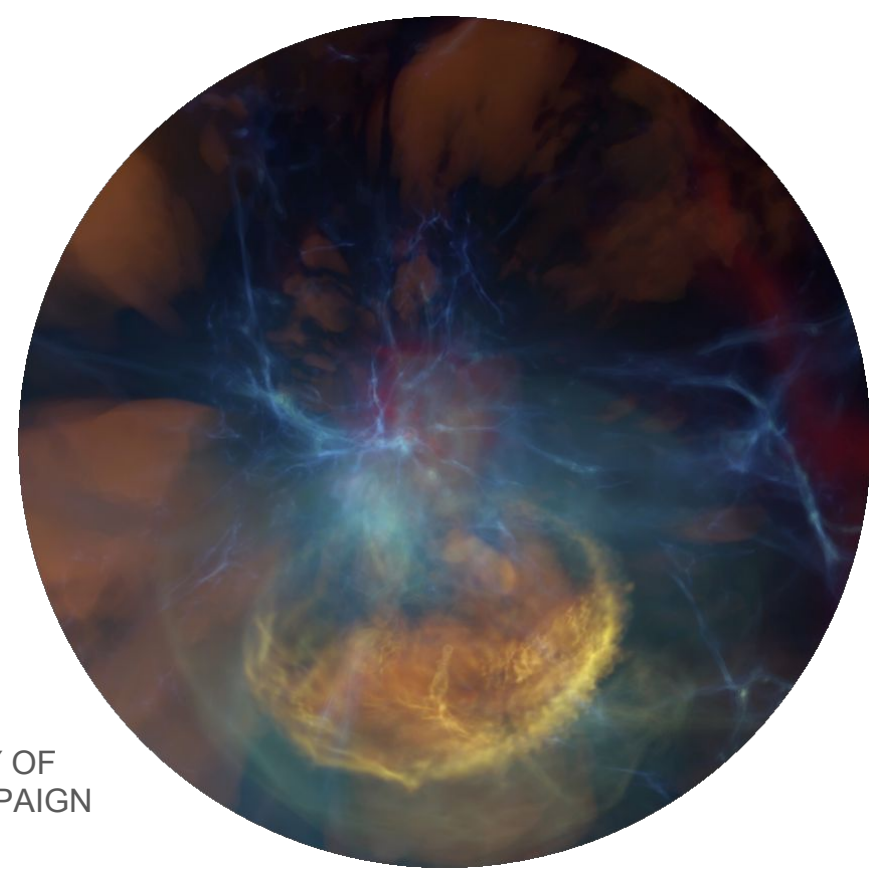

This computer model of a supernova exploding in the early universe was difficult to move from the supercomputer to our local visualization cluster, so we built a special computer graphics pipeline on the supercomputer to create the images directly where the data lived.

Image Credits:

"Solar Superstorms": A full-dome production by NCSA, University of Illinois, Thomas Lucas Productions, Spitz Creative Media

"The Formation of First Stars and Galaxies" simulation: John H. Wise (Georgia Institute of Technology, Center for Relativistic Astrophysics)

Visualization by: Advanced Visualization Lab, National Center for Supercomputing Applications, University of Illinois at Urbana-Champaign, Donna Cox, Kalina Borkiewicz, Jeff Carpenter, AJ Christensen, Stuart Levy, Robert Patterson yt visualization toolkit for volumetric data: Matt Turk, Research Scientist at the National Center for Supercomputing Applications Research, Assistant Professor in the Astronomy department, University of Illinois at Urbana-Champaign Funded in part by: National Science Foundation Award ACl-1445176, The Centrality of Advance Digitally Enabled Science: CADENS 


\section{Cinematic Sci Vis for Outreach}

Cinematic Scientific Visualization is a powerful tool for anyone looking to do fact-based outreach.

Effectively, we're translating "film language" into "science language". "Film language" is something very familiar in our culture, and which learners of all ages can relate to, as kids are typically exposed to family films before they even understand spoken language. In the same vein as "a picture is worth a thousand words", cinematic scientific visualization helps students learn the way science "feels" before they know why it works.

Hollywood-style presentation can influence visualization designers to include visual enhancements like sweeping contextual environments, organic exploratory cinematography, realistic well-designed lighting, and atmospheric depth. We can also include auditory enhancements like descriptive narration, meaningful sound effects, and an emotional score to heighten moments of importance or inspiration. 


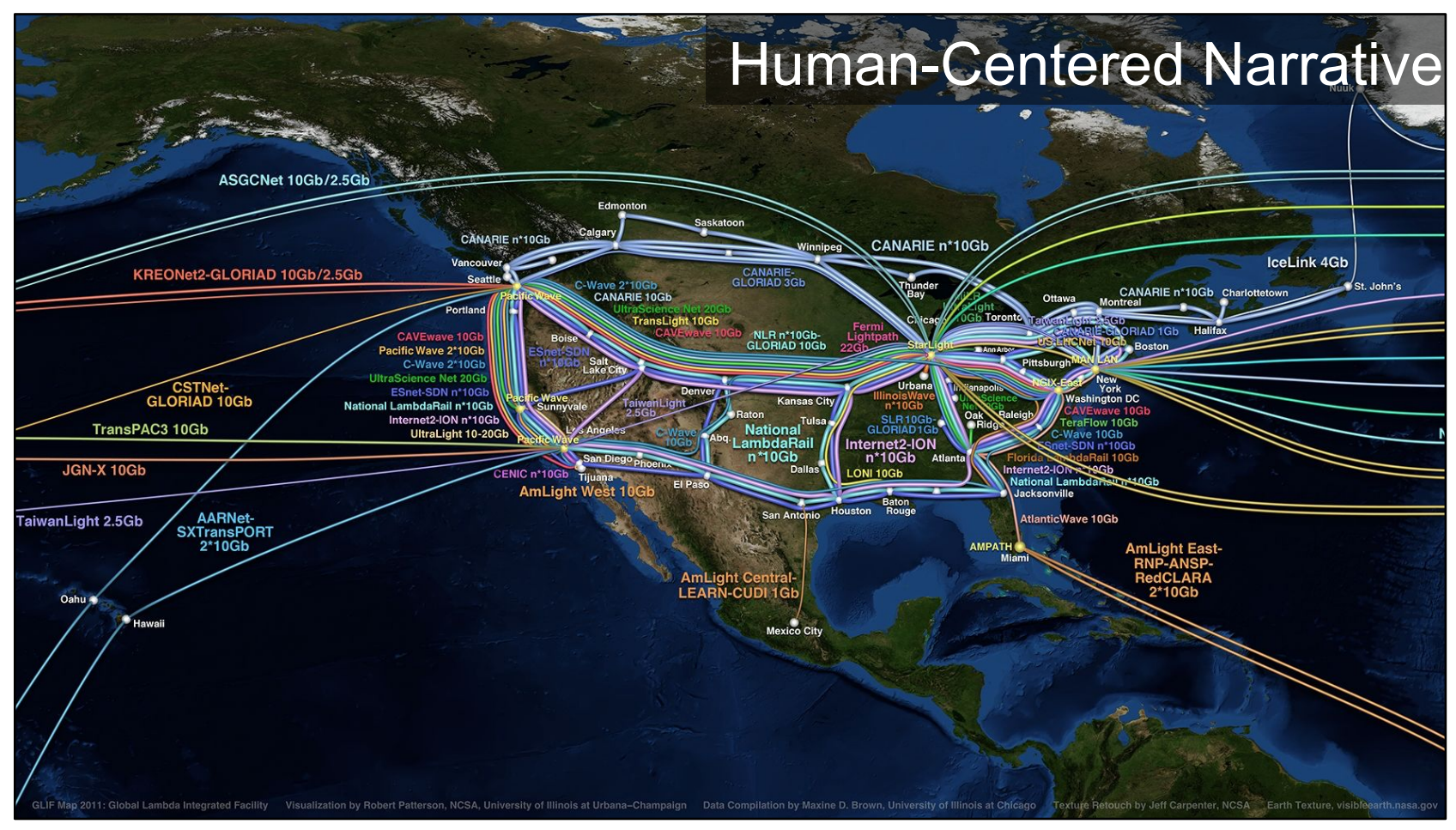

Some of the core features of a Hollywood-style presentation are:

\section{(1) A human-centered narrative}

Hollywood storytellers are experts at evoking emotional reactions to their narratives. This is primarily because no matter how fantastical or distant the setting of the story is, they always center it around characters with human emotions. Science stories often feature characters like galaxies or molecules, so it is important to remind the audience of why they should care about these characters - to think about them in terms of the origins of life, threats to our safety, and human social systems. Science stories can also feature scientists as characters. When scientists are portrayed simply as people who have questions and who design experiments to find answers, it helps audiences think of science as something they can do too.

Image Credits:

Acknowledgements - The Global Lambda Integrated Facility (GLIF) Map 2011 visualization was created by Robert Patterson of the Advanced Visualization Laboratory (AVL) at the National Center for Supercomputing Applications (NCSA) at the University of Illinois at Urbana-Champaign (UIUC), using an Earth image provided by NASA with texture retouching by Jeff Carpenter, NCSA. Data was compiled by Maxine D. Brown of the Electronic Visualization Laboratory (EVL) at the University of Illinois at Chicago (UIC). Support was provided by GLIF, NCSA/UIUC, the State of Illinois, and US National Science Foundation grants \# OCI-0962997 to EVL/UIC. For more information on GLIF, see http://www.glif.is/.

Additional Information - The GLIF map does not represent all the world's Research and Education optical networks, and does not show international capacity that is dedicated to production usage. The GLIF map only illustrates excess capacity that its participants are willing to share with international research teams for applications-driven and computer-system experiments, in full or in part, all or some of the time. GLIF does not provide any network services itself, and researchers should approach individual GLIF network resource providers to obtain lightpath services. 


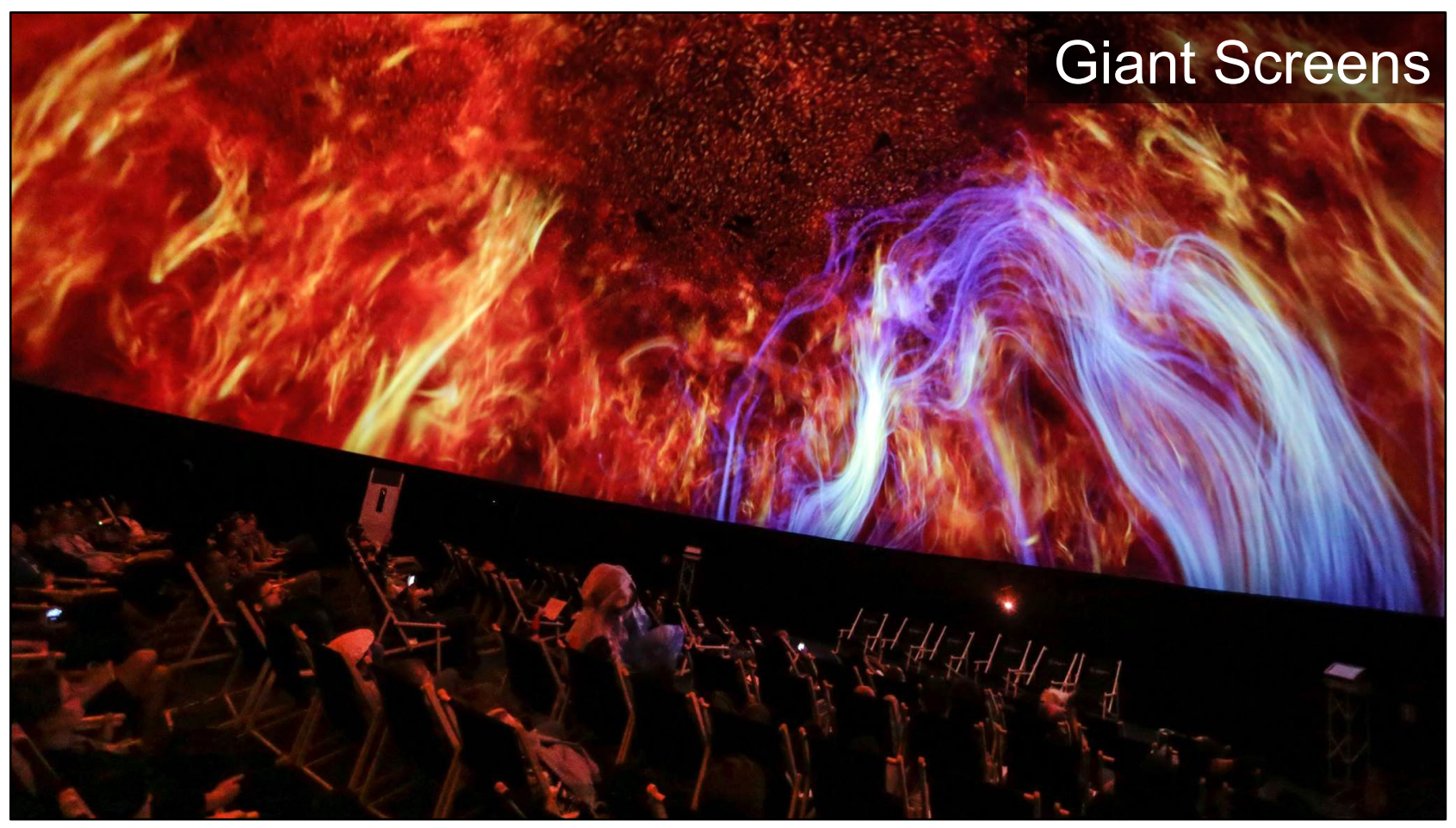

Some of the core features of a Hollywood-style presentation are:

(2) Giant immersive theater

Research shows that embodied learning contributes to better retention and achievement of learning objectives. Immersive IMAX theaters and digital fulldome theaters are venues built to make objects on the screen life-size, and to surround a person's vision to help them disappear into the narrative. It's important to remember that designing for these venues is not like designing for small home screens - objects need to feel large, move smoothly, and have a rich color palette.

Image Credits:

"Solar Superstorms": A full-dome production by NCSA, University of Illinois, Thomas Lucas Productions, Spitz Creative Media

"Magneto-Convection Emerging Flux" simulation: Robert Stein (Physics and Astronomy Department, Michigan State University), Åke Nordlund (Niels Bohr Institute, Copenhagen University, Copenhagen,Denmark, NASA grants NNX08AH44G and NNX12AH49G and NSF grants AGS 1141921 and OCl 1144506), Patrick Moran (NASA Advanced Supercomputing Division, NASA Ames Research Center)

"Active Region Scale Flux Emergence on the Sun" simulation: Matthias Rempel (High Altitude Observatory, National Center for Atmospheric Research), M.C.M. Cheung (Lockheed Martin Solar and Astrophysics Lab)

Visualization by: Advanced Visualization Lab, National Center for Supercomputing Applications, University of Illinois at Urbana-Champaign, Donna Cox, Kalina Borkiewicz, Jeff Carpenter, AJ Christensen, Stuart Levy, Robert Patterson Funded in part by: National Science Foundation Award ACl-1445176, The Centrality of Advance Digitally Enabled Science: CADENS 


\section{Sound Effects}

Some of the core features of a Hollywood-style presentation are:

(3) Carefully designed sound effects and score

Moviemakers know that sound design is even more important than visual design - a poor soundtrack will break an audience's suspension of disbelief instantly. Sounds provide context clues for objects that are on screen and off screen; it gives the audience a different way to observe the objects on the screen; and it helps them focus their attention on salient details.

A film score is another important part of the soundtrack, helping audiences to connect emotionally to the awe-inspiring visuals.

Image credits:

"Hubble 3D" Copyright 2010 Warner Bros. Courtesy of Warner Bros. and IMAX Corporation. 


\section{Celebrity Narrators}

\section{NARRATOR \\ Benedict Cumberbatch}

Some of the core features of a Hollywood-style presentation are:

(4) The inclusion of familiar celebrities

Celebrities drive box office numbers for Hollywood films. In the same way, celebrity narrators can pull audiences to see a documentary. But celebrities are not scientists and that may help audiences to be more receptive. Scientists are often perceived by the general public to be intellectually unrelatable, which can hamper their ability to have meaningful interactions. While we should definitely work to dispel this misconception of scientists, celebrities are perceived to be more trustworthy, and so the general public is more receptive to facts coming from them.

Image Credits:

"Solar Superstorms": A full-dome production by NCSA, University of Illinois, Thomas Lucas Productions, Spitz Creative Media

"Double Coronal Mass Ejection" simulation: Yuhong Fan (High Altitude Observatory, National Center for Atmospheric Research)

"Active Region Scale Flux Emergence on the Sun" simulation: Matthias Rempel (High Altitude Observatory, National Center for Atmospheric Research), M.C.M. Cheung (Lockheed Martin Solar and Astrophysics Lab)

Solar Dynamics Observational Data: Steele Hill (SOHO/STEREO/SDO Media Specialist, NASA Goddard Space Flight Center)

Visualization by: Advanced Visualization Lab, National Center for Supercomputing Applications, University of Illinois at Urbana-Champaign, Donna Cox, Kalina Borkiewicz, Jeff Carpenter, AJ Christensen, Stuart Levy, Robert Patterson Funded in part by: National Science Foundation Award ACl-1445176, The Centrality of Advance Digitally Enabled Science: CADENS 




It bears mentioning that conveying science in the style of Hollywood films runs the risk of being too much fun and obscuring the scientific message. Audiences must be able to understand what the line between fact and fiction is.

Additionally, educational gaming researchers describe the phenomenon of "chocolate covered broccoli" - the idea that you can try to disguise an educational experience as a game and people will quickly discover they've been deceived. Because of this, they will abandon the game experience sooner than if they were presented with a classical educational experience. In the world of "edutainment" it is best not to disguise educational content, but rather celebrate it. 


\section{Communicating Complex scientific Concepts with Imagery}

Why is cinematic scientific visualization a great tool to convey complex science concepts? 


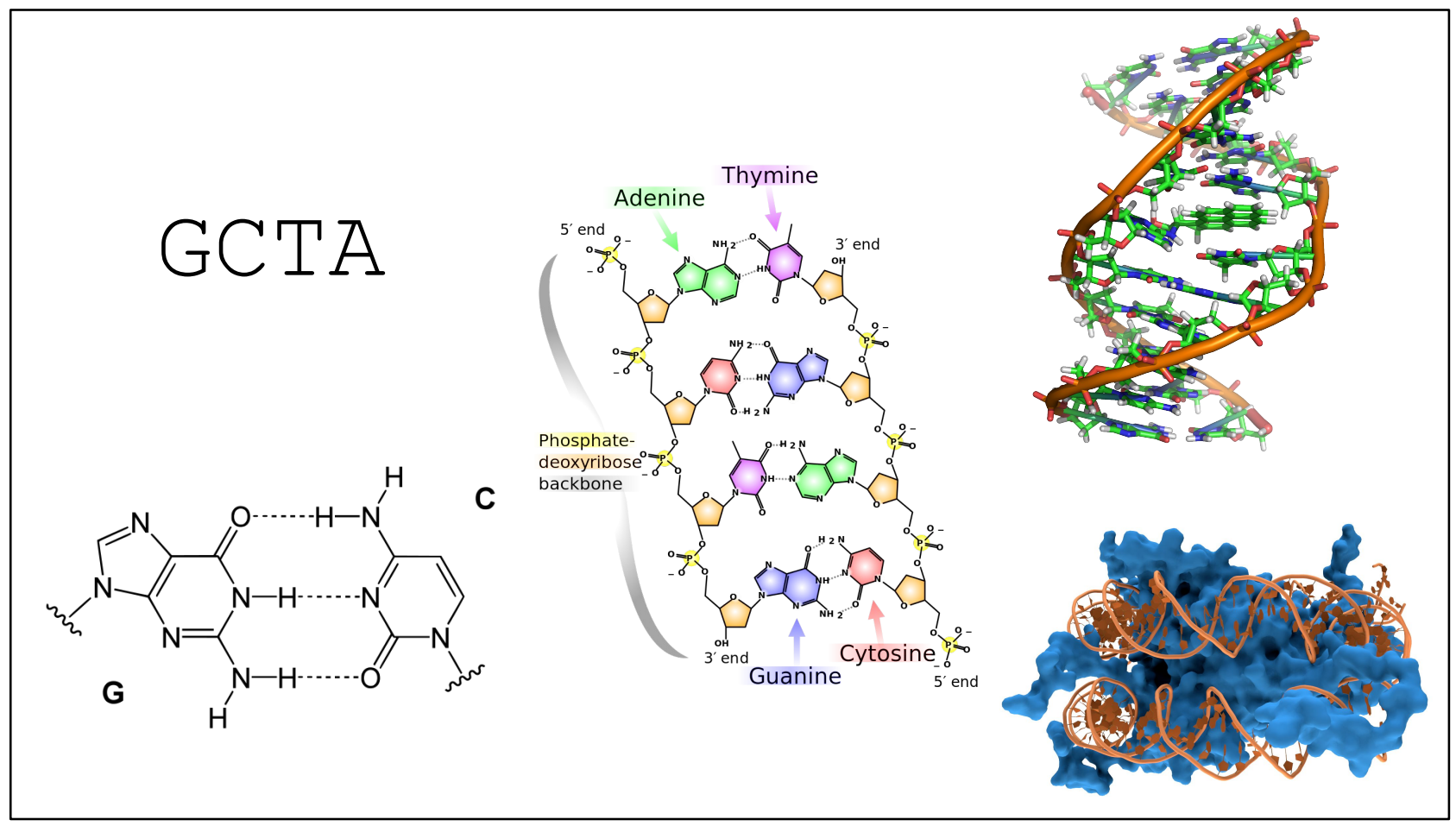

Educational literature refers to "metavisual capability" - the ability to understand a visual abstraction of science, and store that as a mental model that can be built upon and used to extrapolate to other domains.

To build metavisual capability for DNA for instance, you might start by showing a student text describing the structure of DNA, then a 2D diagram, then a 3D ball-and-stick model, then a contextual 3D representation.

Image credits:

Creative commons images courtesy of https://en.wikipedia.org/wiki/DNA 




Cinematic scientific visualization provides another metavisual step beyond the diagrams and abstractions we're used to seeing in textbooks. The difference from these more simplified images is that it puts the science in context. (Remember: alternative facts are most effective when taken out of context.)

Cinematic scientific visualization gives us a starting point for a discussion about phenomena that are a direct result of the geometric time-evolving nature of the physical universe. It builds audience intuition for concepts that are difficult to abstract effectively, such as vast differences in scale, dynamic fluid flow, the entropy of the universe, and gravitational or particle interaction.

Image credits:

Creative commons images courtesy of https://en.wikipedia.org/wiki/Tropical_cyclone;

https://en.wikipedia.org/wiki/Galaxy 


\section{Tools tor visuliatiation}

A Renaissance Team should have an arsenal of tools to be used to track down and present the complexities of the data with which they want to communicate. 


\section{Tools for Pre-Visualization: Data Pre-Vis Software, Partiview}

Pre-visualization is essential to create immersive and deliberate camera moves through complex dynamic datasets. If the size of each data timestep file is large and takes seconds or minutes to load, there is no easy way to preview unfiltered temporal dynamics. Thus, we need to sample down the data so that an interactive rendering of a sequence of data timesteps is possible.

We usually use a subset of all data timesteps and reduce each timestep's memory footprint so the data sequence fits within the memory of our interactive $4 \mathrm{~K}$ stereoscopic display system, and so that it takes a reasonable amount of time for loading. The goal is to have enough visual information and temporal dynamics to compose the frame and create camera moves for exporting and rendering with the full data set. We also use pre-vis to dial in the desired data rate, set the overall length of the scene, and experiment with different representations of the data. 


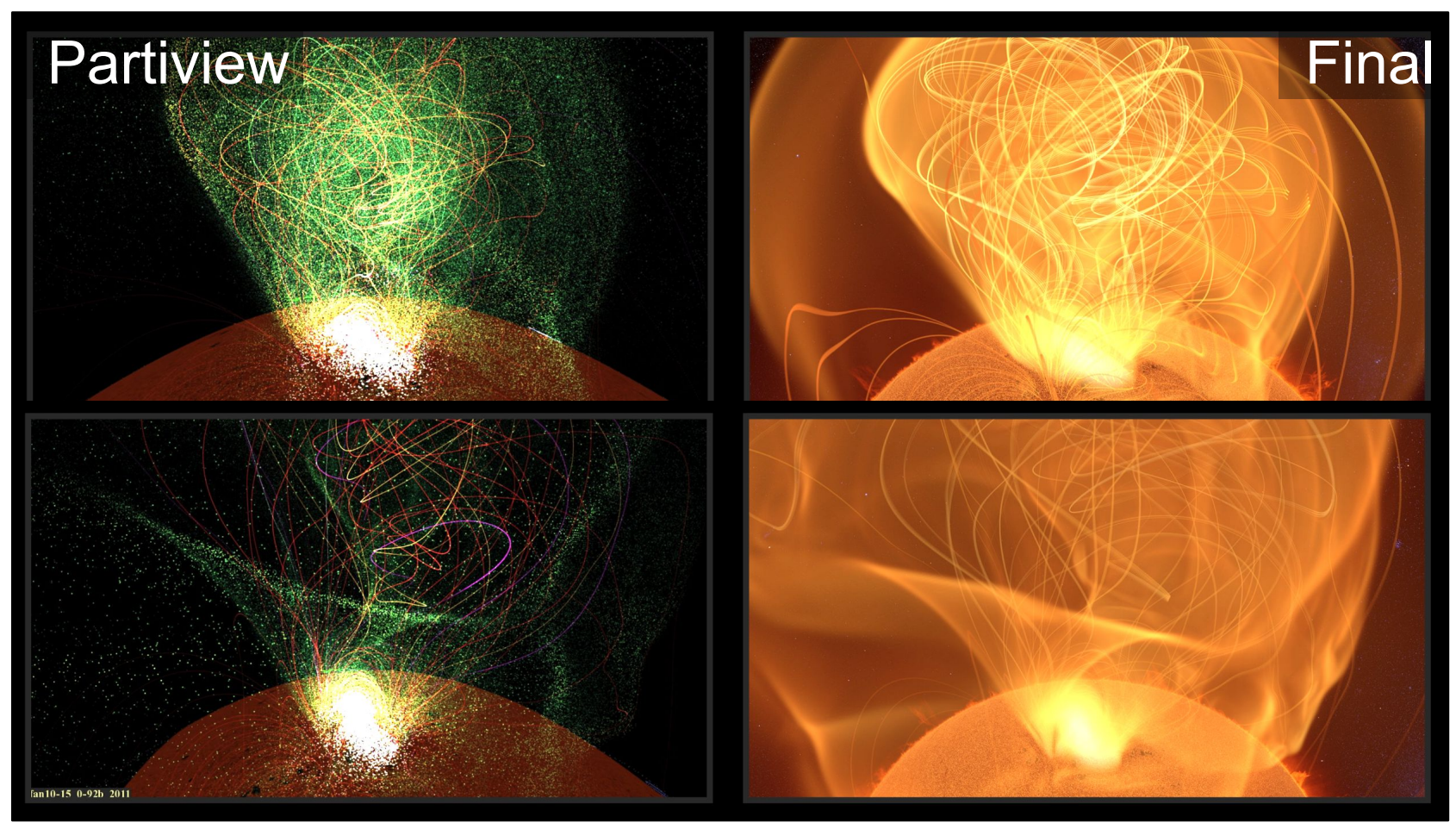

Partiview is a customizable interactive data renderer maintained by the AVL and lead developer Stuart Levy. It is free and open source, and available at

http://virdir.ncsa.illinois.edu/partiview.

Partiview is capable of efficiently rendering data represented as star-like particles, lines, and triangles. Previewing the data at the speed it will be in the final movie is imperative for decisions about timing, and so we subsample the enormous data sets we work with into the geometry types that Partiview can render. Because our team frequently works with volumetric data, we often sample point clouds from the volumes to make a representation of the data that is able to update and render many times a second.

On the left we see a Partiview pre-visualization of a coronal mass ejection data simulation, and on the right is the final rendering.

Image Credits:

"Solar Superstorms": A full-dome production by NCSA, University of Illinois, Thomas Lucas Productions, Spitz

Creative Media

"Double Coronal Mass Ejection" simulation: Yuhong Fan (High Altitude Observatory, National Center for Atmospheric Research)

"Active Region Scale Flux Emergence on the Sun" simulation: Matthias Rempel (High Altitude Observatory, National Center for Atmospheric Research), M.C.M. Cheung (Lockheed Martin Solar and Astrophysics Lab) SDO Data: Steele Hill (SOHO/STEREO/SDO Media Specialist, NASA Goddard Space Flight Center)

Visualization by: Advanced Visualization Lab, National Center for Supercomputing Applications, University of Illinois at Urbana-Champaign, Donna Cox, Kalina Borkiewicz, Jeff Carpenter, AJ Christensen, Stuart Levy, Robert Patterson Funded in part by: National Science Foundation Award ACl-1445176, The Centrality of Advance Digitally Enabled Science: CADENS 


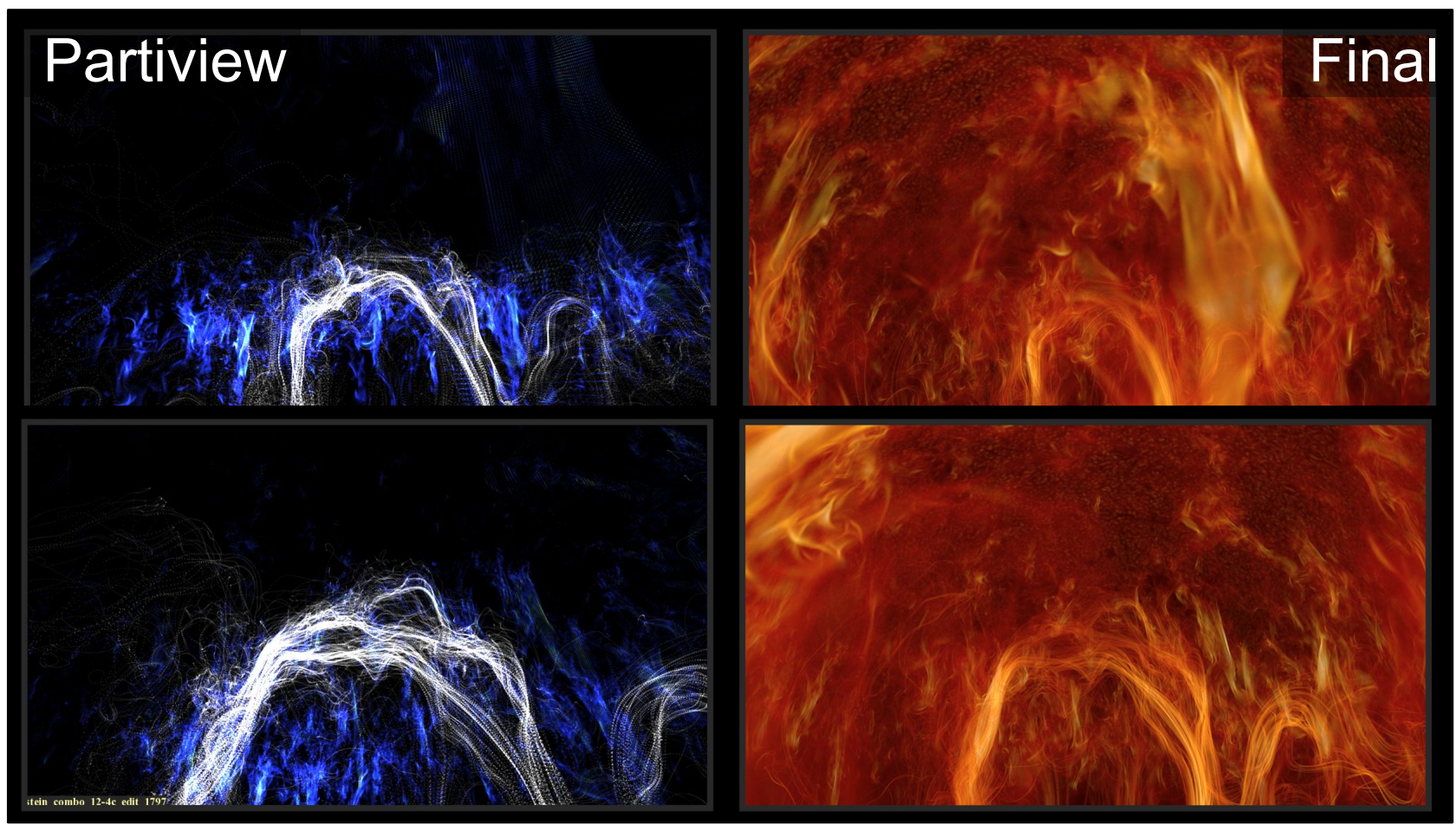

Pre-visualization of unfamiliar data in Partiview saves time and effort - its extreme flexibility for different types of data makes it ideal for experimenting with timing, mining the data for shapes and features, and getting approval from domain experts.

On the left we see a pre-visualization of rising arcs of magnetic field lines just below the sun's surface, and on the right is a raytraced volume rendering.

Image Credits:

"Solar Superstorms": A full-dome production by NCSA, University of Illinois, Thomas Lucas Productions, Spitz

Creative Media

"Magneto-Convection Emerging Flux" simulation: Robert Stein (Physics and Astronomy Department, Michigan State University), Åke Nordlund (Niels Bohr Institute, Copenhagen University, Copenhagen,Denmark, NASA grants NNX08AH44G and NNX12AH49G and NSF grants AGS 1141921 and OCl 1144506), Patrick Moran (NASA Advanced Supercomputing Division, NASA Ames Research Center)

"Active Region Scale Flux Emergence on the Sun" simulation: Matthias Rempel (High Altitude Observatory, National Center for Atmospheric Research), M.C.M. Cheung (Lockheed Martin Solar and Astrophysics Lab)

Visualization by: Advanced Visualization Lab, National Center for Supercomputing Applications, University of Illinois at Urbana-Champaign, Donna Cox, Kalina Borkiewicz, Jeff Carpenter, AJ Christensen, Stuart Levy, Robert Patterson Funded in part by: National Science Foundation Award ACl-1445176, The Centrality of Advance Digitally Enabled Science: CADENS 




Partiview scenes can become quite complex and so it's important to put effort into their design as much as the production rendering software. Partiview allows you to adjust particle and line sizes, colors, and opacities based on data attributes, camera distance, and more.

On the left we see a pre-visualization of a simulation of the sun, and on the right a raytraced volume rendering.

Image Credits:

"Solar Superstorms": A full-dome production by NCSA, University of Illinois, Thomas Lucas Productions, Spitz Creative Media

"Solar Dynamo / Solar Interior" simulation by: Juri Toomre (JILA, University of Colorado Boulder), Mark Miesch (High Altitude Observatory, National Center for Atmospheric Research), Nicholas Nelson (Los Alamos National Laboratory), Allan Sacha Brun (Commissariat à l'énergie atomique et aux énergies alternatives, Saclay, France), Benjamin Brown (Laboratory for Atmospheric and Space Physics, University of Colorado Boulder)

Visualization by: Advanced Visualization Lab, National Center for Supercomputing Applications, University of Illinois at Urbana-Champaign, Donna Cox, Kalina Borkiewicz, Jeff Carpenter, AJ Christensen, Stuart Levy, Robert Patterson Funded in part by: National Science Foundation Award ACl-1445176, The Centrality of Advance Digitally Enabled Science: CADENS 


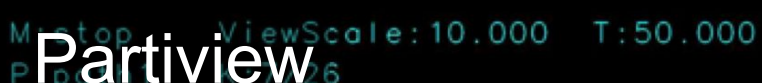

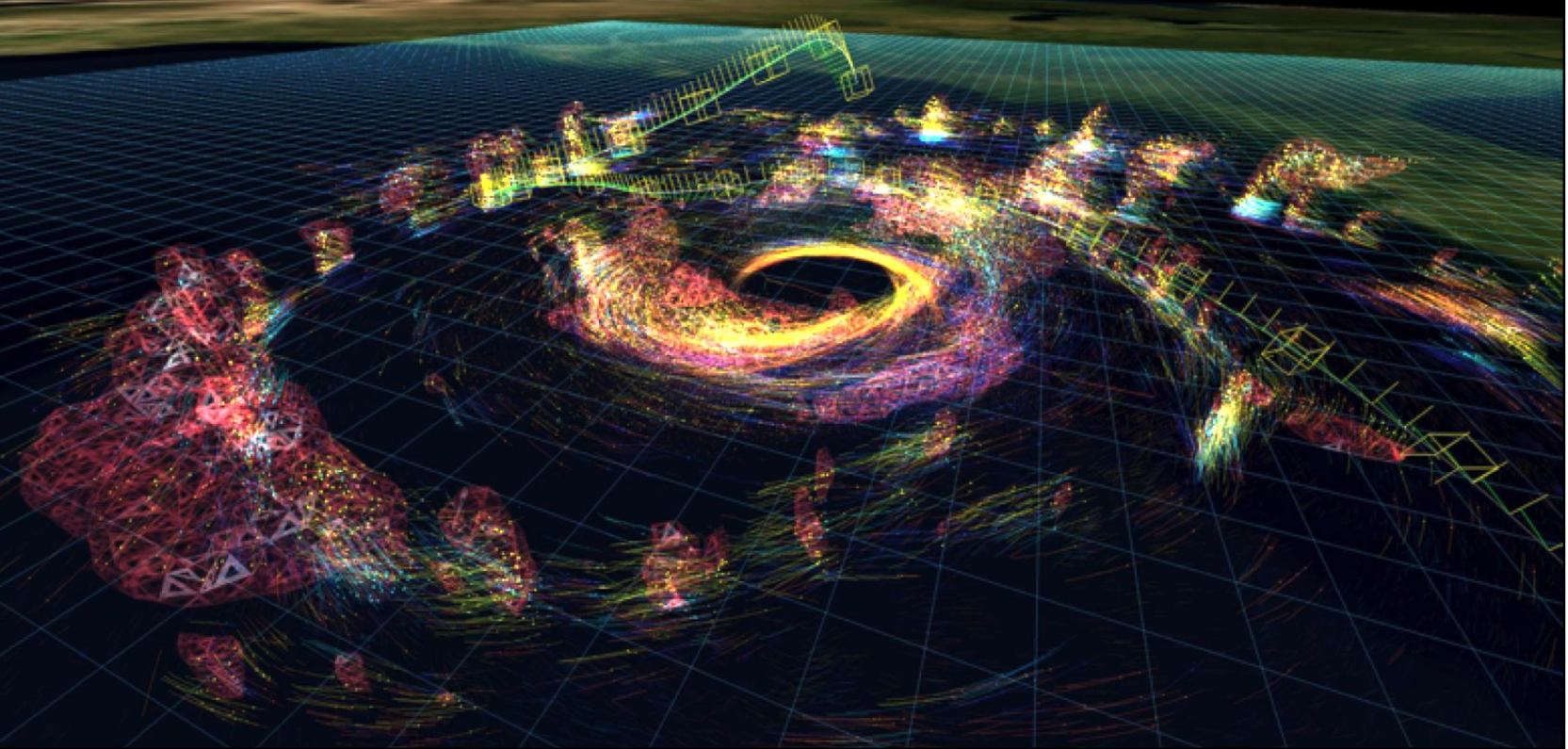

This previsualization of a hurricane simulation combines many of Partiview's features particles advected in a wind field, lines that are proxies for streamtubes, and triangle meshes representing isosurfaces of clouds. This interactive scene allowed AVL camera choreographer Bob Patterson to get very close to the hurricane eye wall without colliding with geometry. 


\section{Tools for Data Exploration: OurVR Software, Virtual Director}

Virtual Director is a real time remote-collaborative data analysis application that was originally written for a virtual reality environment called the CAVE in the mid 90's. We have since adapted it to run in other environments, including our $4 \mathrm{~K}$ stereo theater where we prototype IMAX and fulldome films. 


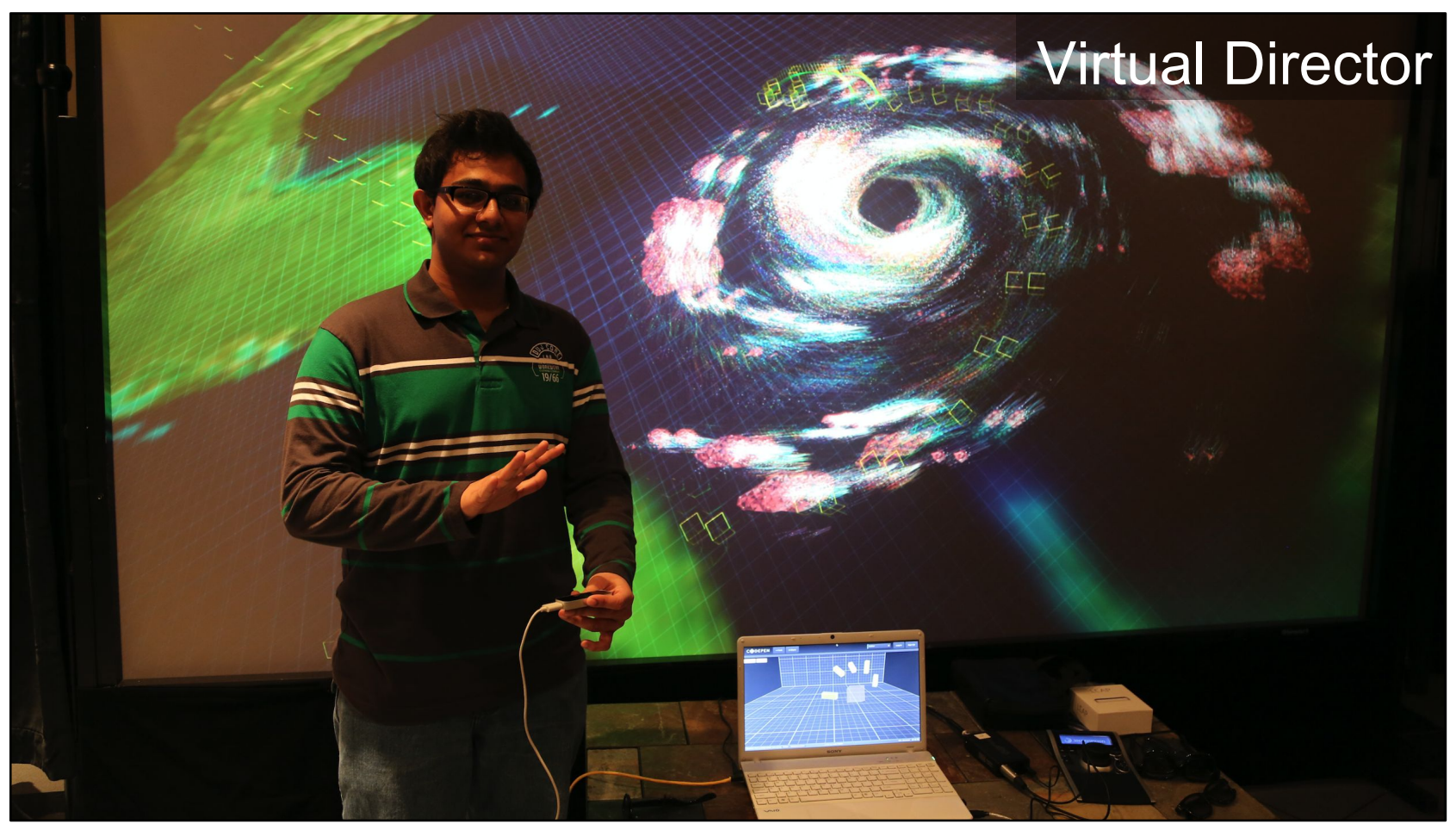

Virtual Director is a tool that was developed at the NCSA for the purposes of data exploration and cinematic camera choreography. It accepts voice and text commands for many of the functions like controlling the display and triggering wand gesture input. It links up with real time rendering engines (like Partiview) and provides navigational controls for organically manipulating the viewpoint with 6-degree-of-freedom devices like the SpacePilot 3D mouse, the LEAP Motion hand tracker, and magnetic wands. These un-tethered control devices allow us to "perform" camera moves through the data in a way familiar to live action cinematographers - and this technology from the NCSA influenced the development of related tools in Hollywood VFX studios. 




In addition to being able to represent data as particles, lines, and triangles in Partiview, we have also developed a renderer that can show textured Maya geometry in real time.

Virtual Director is also an editing tool - camera paths are represented with keyframes along a spline which can be edited in real time. A secondary "television" window can be used to show the performed camera's animating viewpoint while the main scene view remains fully interactive.

The camera path spline is then exported in an ASCII format either as relatively sparse spline keyframes or baked so that each frame is a keyframe, both of which are readable by other VFX tools, scientific tools, and our custom renderers. 


\section{Virtual Director}

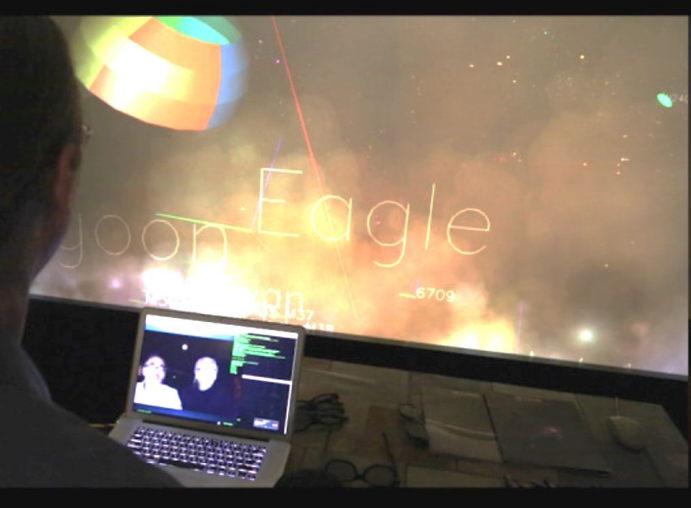

NCSA Lab - Urbana

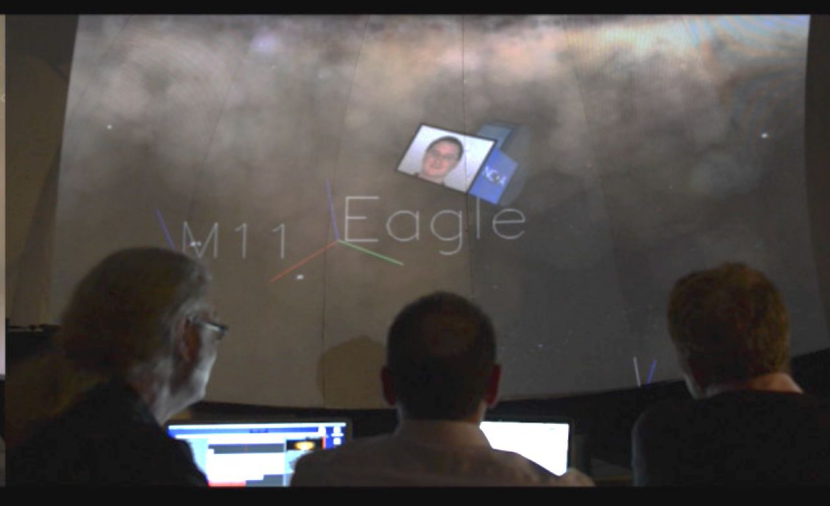

Adler Mini-Dome - Chicago

One of the most useful features of Virtual Director is that it is remotely collaborative. Many participants can join a virtual session from different geographic locations, and be seen as custom avatars to other participants. This allows us to work directly with our clients at science centers and domain experts where they live to get immediate feedback about what we should be targeting. 


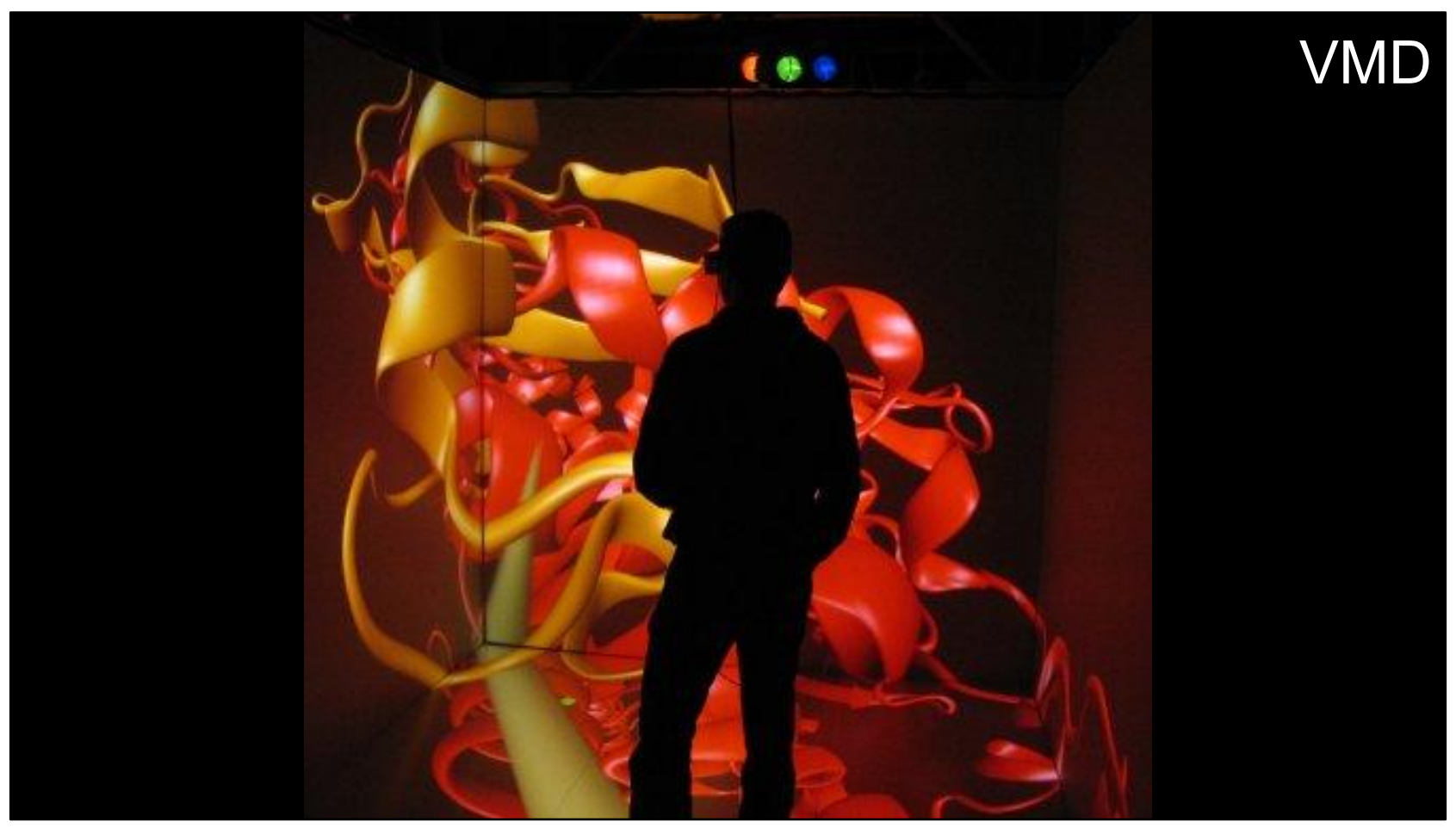

VMD is an interactive scientific visualization tool that we describe in more detail later, but which was another application to run in the CAVE virtual reality environment in the 90's. This kind of data exploration process is widely appreciated by domain experts. 


\section{Tools for Visual Effects: \\ The Commercial Software, Houdini}






Houdini, a commercial visual effects software, is the main tool we use for look development and rendering (with Mantra). Houdini is available at https://sidefx.com/

The AVL writes custom plugins and scripts to allow Houdini to work with scientific data. This includes tools for data loading and resampling, as well as artistic tools such as smooth falloff, isosurfaces, and spatial and temporal interpolation.

The Houdini+Mantra combination works well in rendering multiple embedded high-resolution volumes efficiently and beautifully.

Simulation Credits:

"El Reno 2011 Storm" simulation: Leigh Orf, University of Wisconsin 


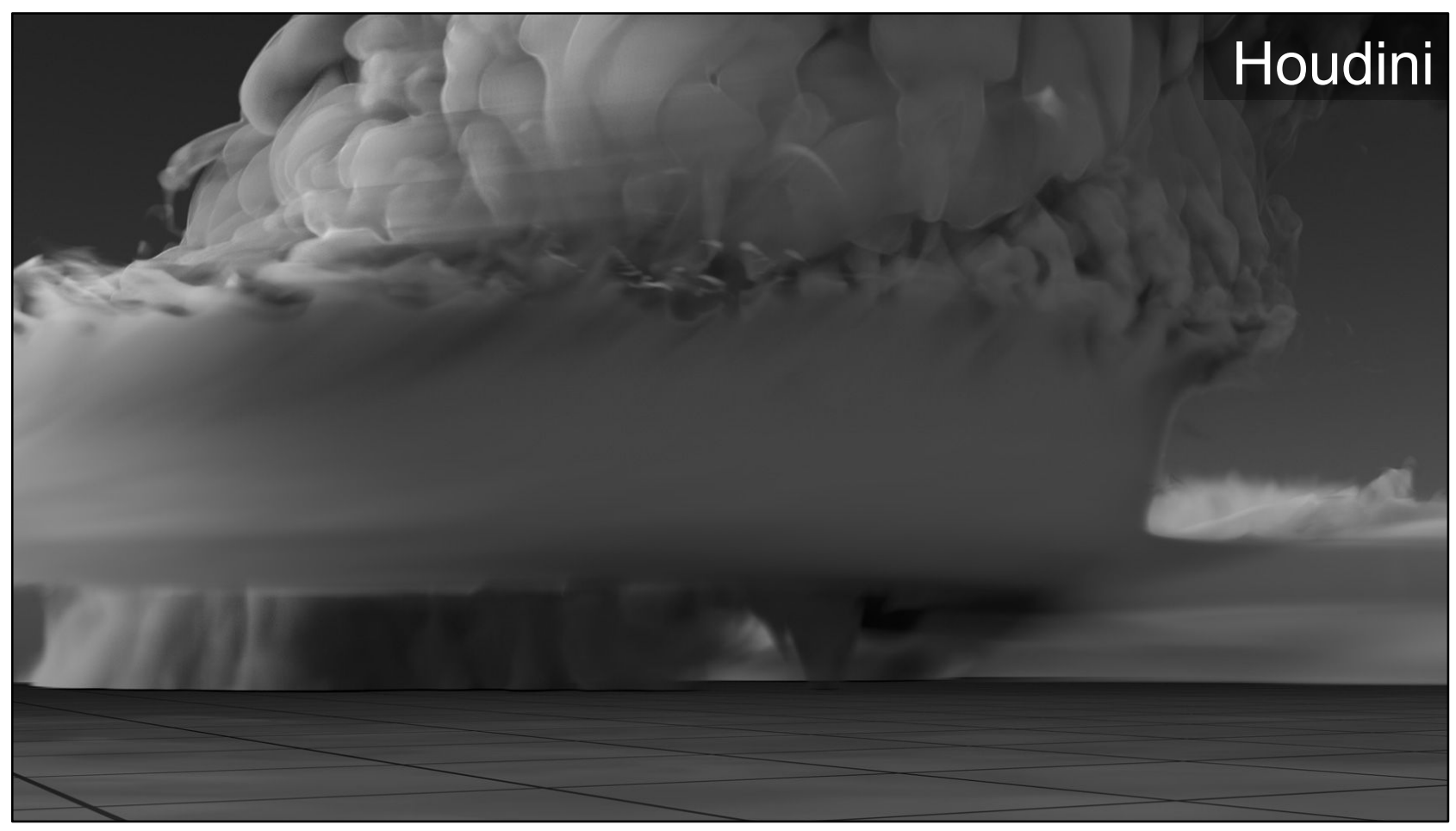

Here is a final rendering of tornado data, integrating a high-resolution VDB sparse volume adapted from a non-uniform grid on top of a geometric ground plane, done with Houdini and Mantra.

Image Credits:

"El Reno 2011 Storm" simulation: Leigh Orf, University of Wisconsin

Visualization by: Advanced Visualization Lab, National Center for Supercomputing Applications, University of Illinois at Urbana-Champaign, Donna Cox, Kalina Borkiewicz, Jeff Carpenter, AJ Christensen, Stuart Levy, Robert Patterson, funded in part by: National Science Foundation Award ACl-1445176, The Centrality of Advance Digitally Enabled Science: CADENS 


\section{Data Grids}

Houdini expects data to look like this:

\begin{tabular}{|l|l|l|l|l|l|}
\hline & & & & & \\
\hline & & & & & \\
\hline & & & & & \\
\hline & & & & & \\
\hline & & & & & \\
\hline & & & & & \\
\hline
\end{tabular}

But often scientific data looks like this:

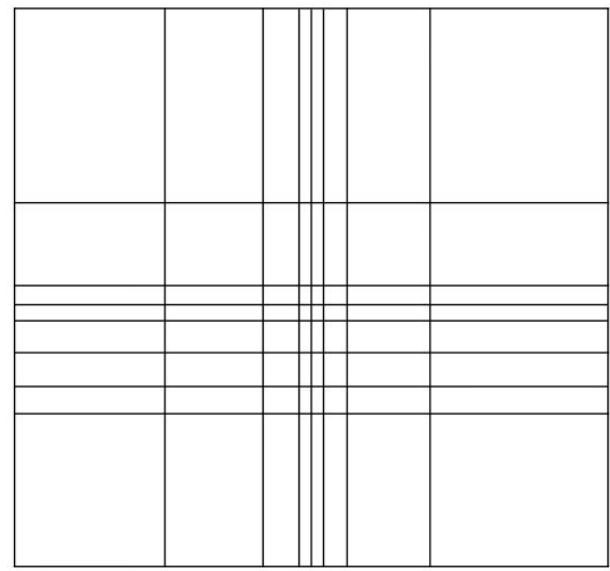

Houdini expects volumetric data to be in a uniform, cartesian grid. However, this often not an efficient way to store scientific data. The tornado data came in a stretched grid... 


\section{Data Grids}

Houdini expects data to look like this:

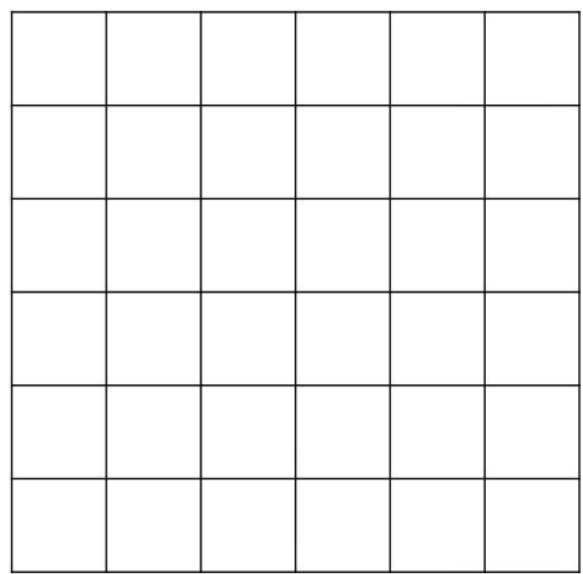

But often scientific data looks like this:

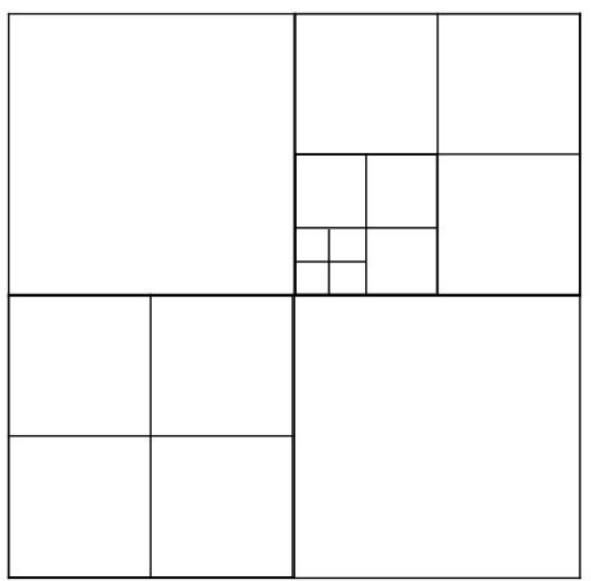

...or, data can come in a nested, multi-resolution grid... 


\section{Data Grids}

Houdini expects data to look like this:

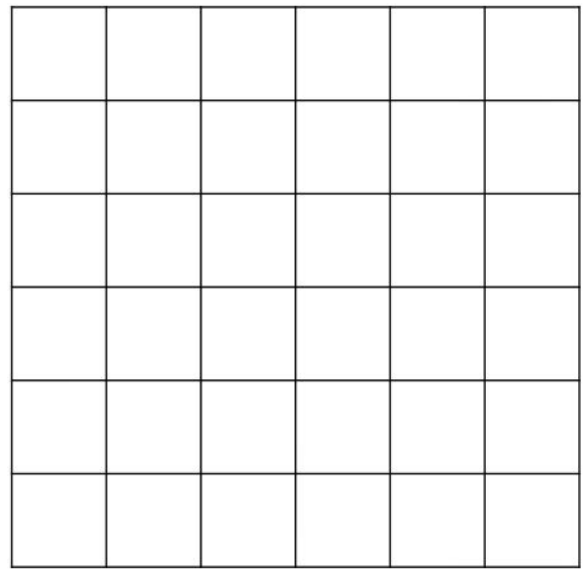

But often scientific data looks like this:



...or a spherical grid, or other non-cartesian and/or non-uniform data formats. 
Houdini expects data to look like this:

But often scientific data looks like this:


Possible solutions:

A. Up-res and interpolate? $\longrightarrow$ Wastes memory.

B. Sample down into grid? $\longrightarrow$ Loses data.

C. Use different software? $\rightarrow$ Has pros and cons...

To be compatible with Houdini, we would need to force the data to fit into a uniform, cartesian grid. This can be done by either:

A. sampling the data into a uniform grid which matches the finest resolution of the data, by "up-resing" the low-resolution areas, and interpolating to get the values, or

$B$. sampling the data into a reasonably-sized uniform grid, matching a medium-sized cell size in the original data, by "up-resing" in lower-resolution areas and "down-resing" in higher-resolution areas.

Option A can easily become unmanageable due to memory and space constraints, if there is a large difference between the largest and smallest cell sizes. Also, this requires interpolation, which creates data that isn't really in the simulation.

Option B is sometimes a good compromise, but has some of the same drawbacks as Option $A$, as well as the fact that we are losing data in the high-resolution areas of the simulation, which is often where the most interesting dynamics are happening.

While we usually do some combination of these options, there is a third option:

Foregoing Houdini altogether. 


\section{Tools for Science: The Python Package, yt}

Scientists have spent decades writing some really great computational tools too! 


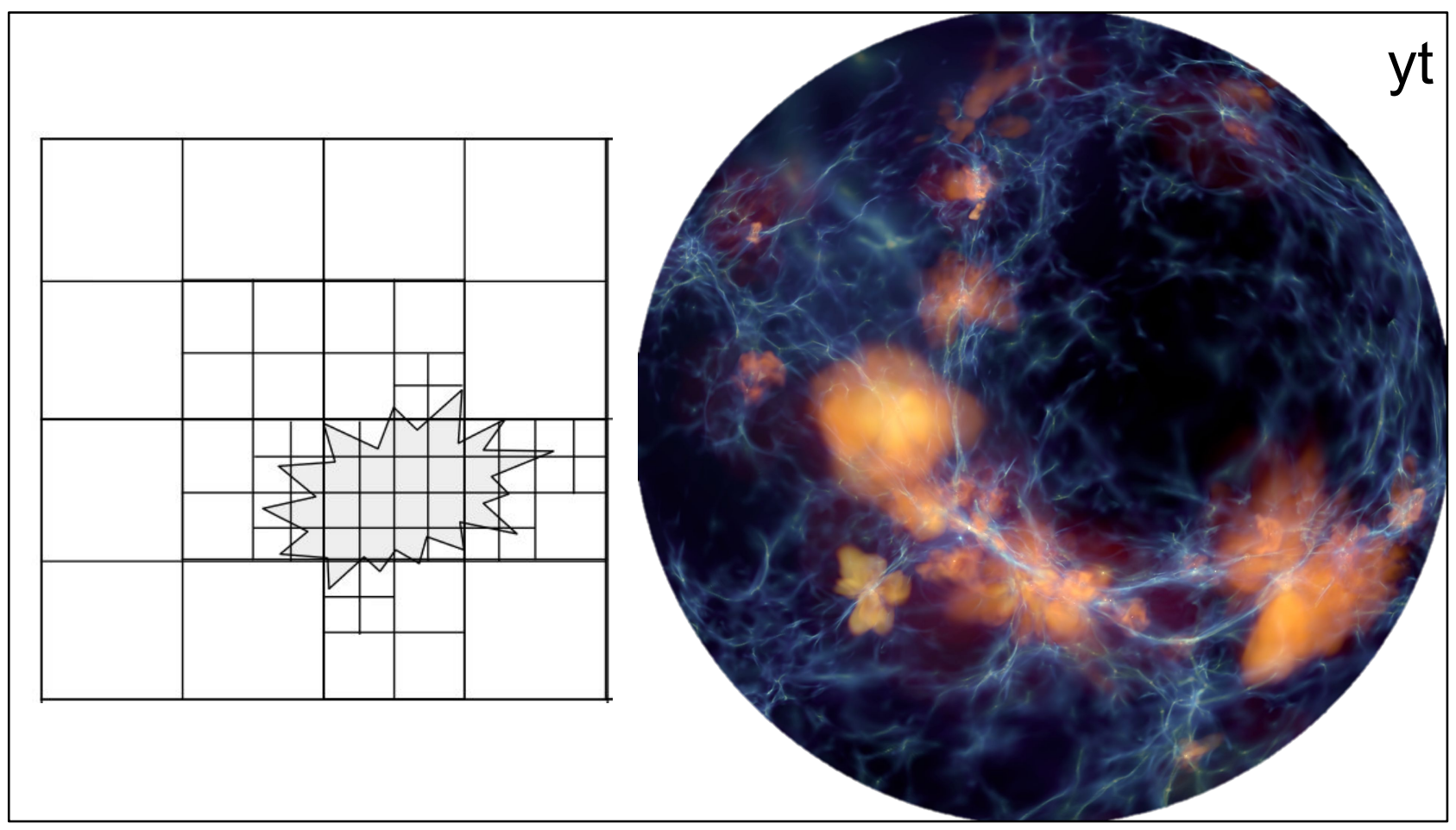

Like there are many options for VFX software (Houdini, Maya, Blender, etc), there are also many options for scientific visualization software (yt, VMD, Vislt, ParaView, etc). In this course, we will describe two of these - yt and VMD.

$\mathrm{Yt}$ is a scientific analysis and visualization Python package. It is the main scientific visualization tool we have used for visualization of complex astrophysical data formats, such as nested multi-resolution grids as described in previous slides. This data format is called Adaptive Mesh Refinement (AMR), and yt can read and render this natively. The image on the right is an AMR data set rendered with yt.

Yt is free and open-source and available at http://yt-project.org/

Image Credits:

"Solar Superstorms": A full-dome production by NCSA, University of Illinois, Thomas Lucas Productions, Spitz Creative Media

"The Formation of First Stars and Galaxies" simulation: John H. Wise (Georgia Institute of Technology, Center for Relativistic Astrophysics)

Visualization by: Advanced Visualization Lab, National Center for Supercomputing Applications, University of Illinois at Urbana-Champaign, Donna Cox, Kalina Borkiewicz, Jeff Carpenter, AJ Christensen, Stuart Levy, Robert Patterson yt visualization toolkit for volumetric data: Matt Turk, Research Scientist at the National Center for Supercomputing Applications Research, Assistant Professor in the Astronomy department, University of Illinois at Urbana-Champaign Funded in part by: National Science Foundation Award ACl-1445176, The Centrality of Advance Digitally Enabled Science: CADENS 


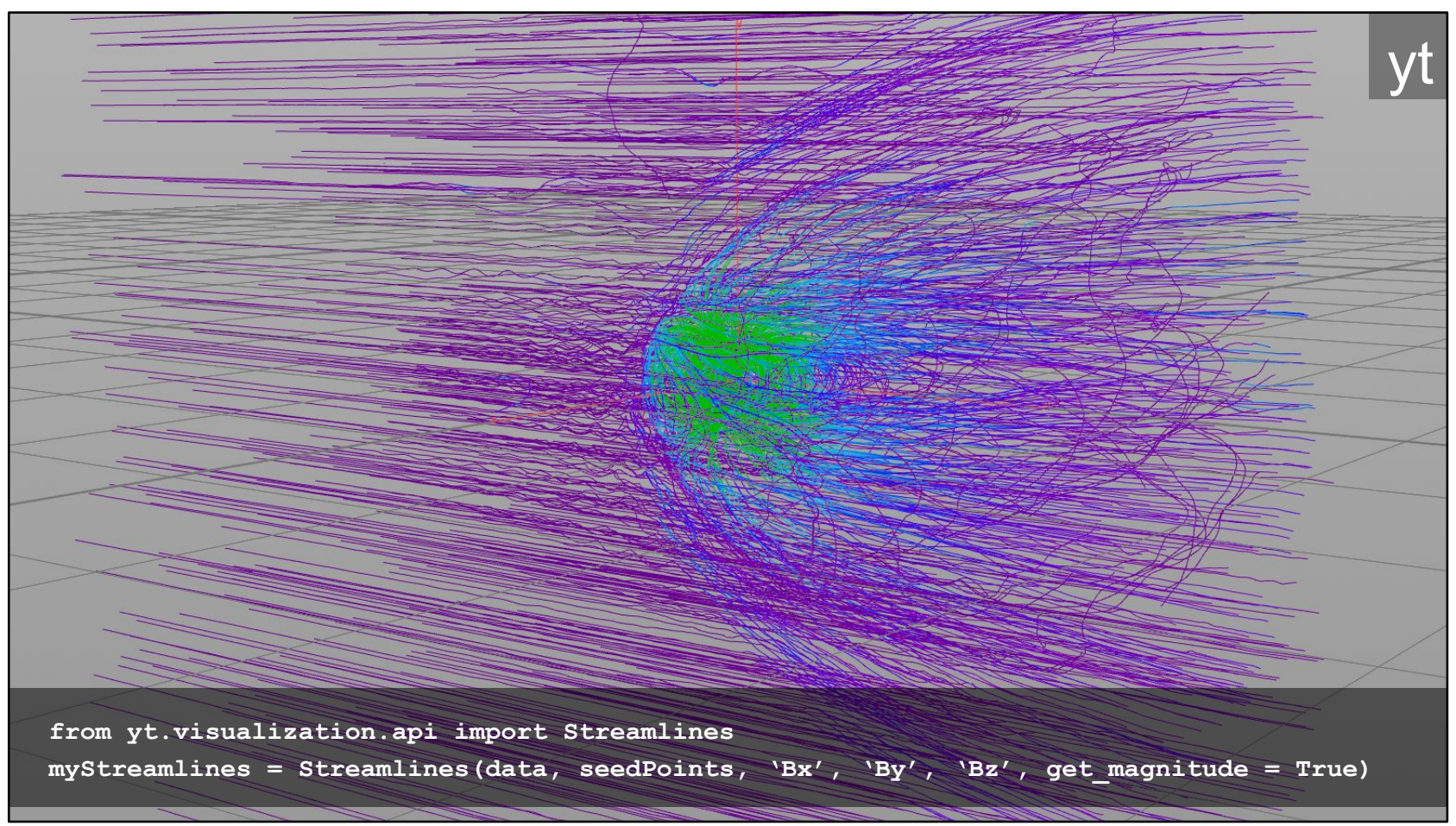

Yt is a tool aimed at scientists more so than at visualization experts. As such, it includes many features useful to scientists, such as tracing streamlines through data. This is something that we often do in Houdini, but yt makes this simpler, just taking a few lines of code. 




Here is the final rendering, done in Houdini, using the magnetic field lines calculated in yt.

Image Credits:

"Solar Superstorms": A full-dome production by NCSA, University of Illinois, Thomas Lucas Productions, Spitz Creative Media

"Solar Plasma Interacting with Earth's Magnetic Field" simulation by Homa Karimabadi (University of California San Diego, Center for Astrophysics and Space Sciences), Mahidhar Tatineni (San Diego Supercomputer Center), Vadim Roytershteyn (Space Science Institute), Hoanh X. Vu (University of California San Diego), Amit Majumdar (San Diego Supercomputer Center)

Visualization by: Advanced Visualization Lab, National Center for Supercomputing Applications, University of Illinois at Urbana-Champaign, Donna Cox, Kalina Borkiewicz, Jeff Carpenter, AJ Christensen, Stuart Levy, Robert Patterson yt visualization toolkit for volumetric data: Matt Turk, Research Scientist at the National Center for Supercomputing Applications Research, Assistant Professor in the Astronomy department, University of Illinois at Urbana-Champaign Funded in part by: National Science Foundation Award ACI-1445176, The Centrality of Advance Digitally Enabled Science: CADENS 


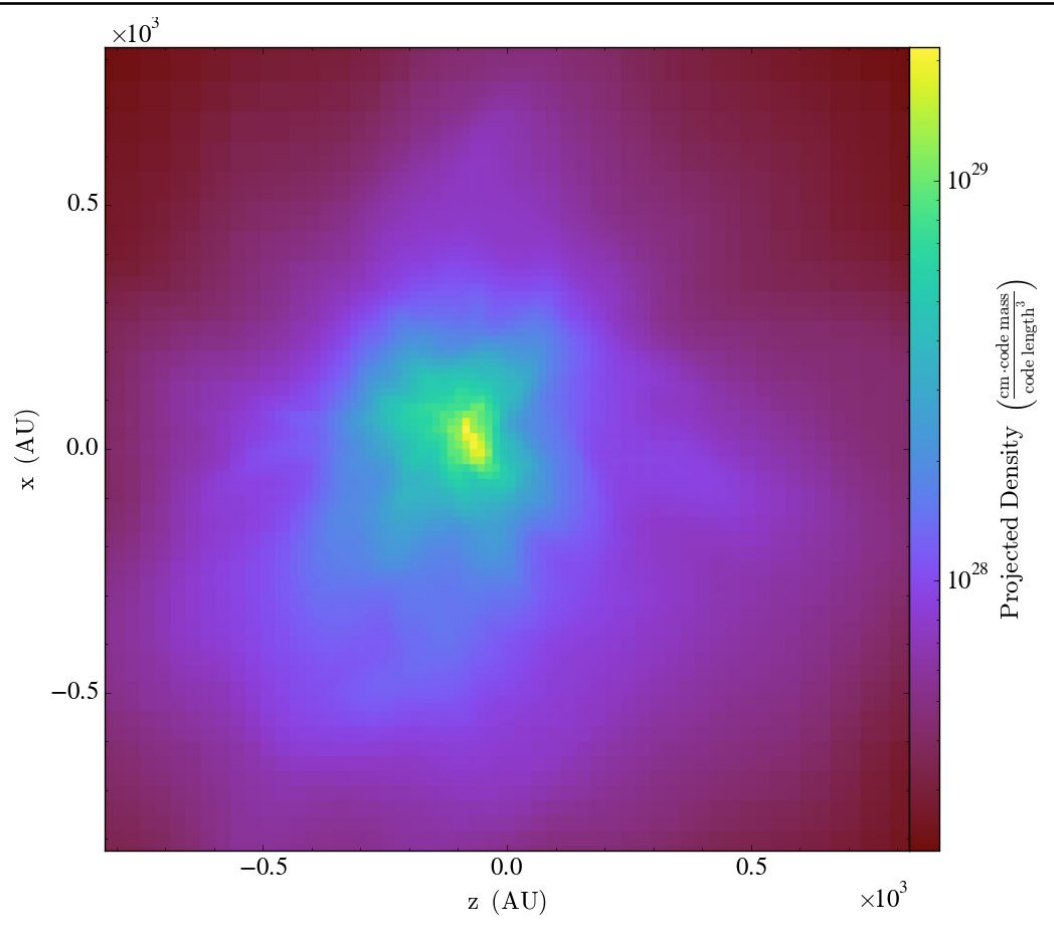

yt has many built-in functions for data analysis. This is useful for data exploration, taking an initial look at the data, and for quickly and easily getting quantitative information about the data, such as positions of regions of interest, or minimum and maximum values. 


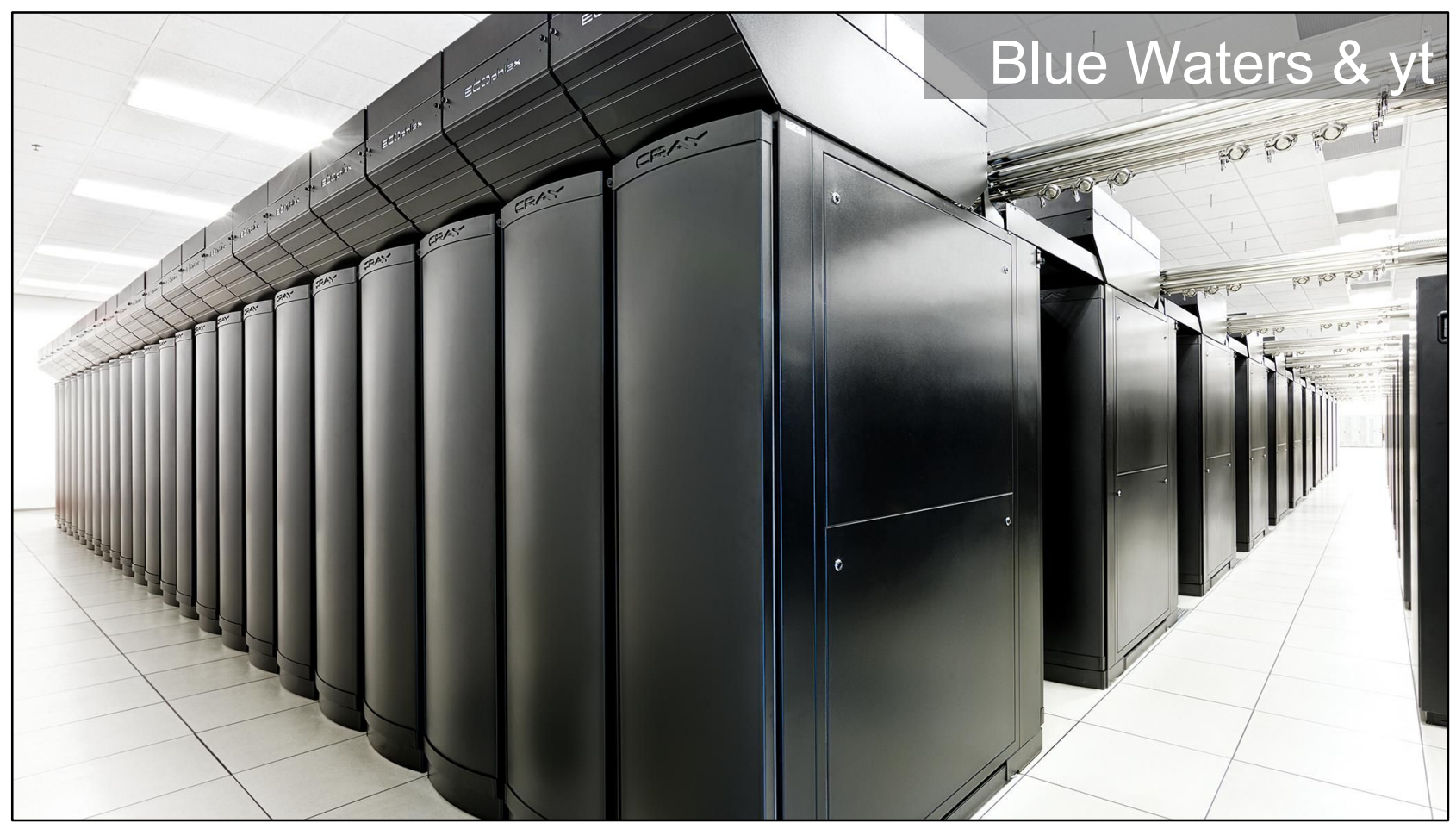

yt works in parallel and is installed on the Blue Waters supercomputer. We have taken advantage of being able to run yt across thousands of cores. We have used as many as 16,327 hours in one day of rendering on Blue Waters (which comes out to 1.9 years!) 




yt is open-source, and one of its primary creators, Dr. Matt Turk, is an NCSA colleague. His team of researchers have helped us develop the software so that it's usable for cinematic rendering. 




So why isn't yt our primary tool?

One downside to yt is that it does not have a user interface. Using yt requires a knowledge of Python, and so it requires artists to be programmers. Camera paths, color maps, and all artistic elements need to be either created programmatically, or created in external software and then imported - both of which require more coding and more work than a more artist-friendly tool like Houdini.

During our work on "Solar Superstorms", due to the large size of the data, we had to wait up to 24 hours for a single frame to finish rendering before we could see whether or not our code worked correctly. This made iteration slow and difficult. However, an experimental interactive volume renderer was recently introduced in last year's release of yt 3.3. 


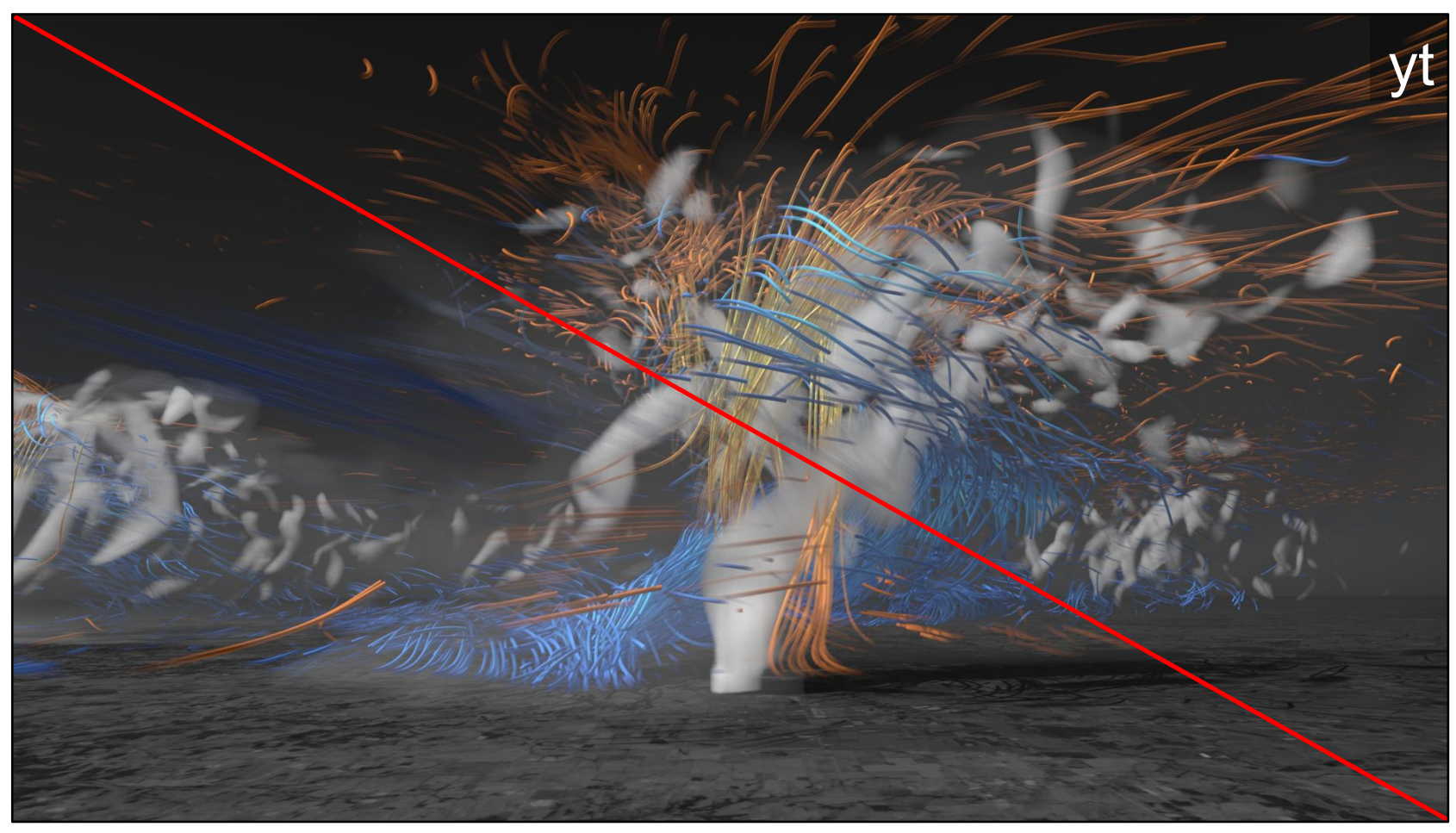

More downsides: Yt only renders volumetric data, and only one object at a time. Yt also doesn't have many fancy settings, such as raytraced lighting and shadows, ambient occlusion, shading and texturing, etc.

It makes shots like this impossible, where a volume is integrated with geometry, with lighting and shadows.

Image Credits:

Joplin 2011 Storm Simulation: Brian Jewett and Kevin Van Leer, University of Illinois at Urbana-Champaign GIS data: Johnathan Rush, Junjun Yin, Aiman Soliman, Dandong Yin, and Shaowen Wang, CyberGIS Center for Advanced Digital and Spatial Studies, Funded in part by the National Science Foundation Awards: 1047916 and 1443080

Visualization by: Advanced Visualization Lab, National Center for Supercomputing Applications, University of Illinois at Urbana-Champaign, Donna Cox, Kalina Borkiewicz, Jeff Carpenter, AJ Christensen, Stuart Levy, Robert Patterson, funded in part by: National Science Foundation Award ACl-1445176, The Centrality of Advance Digitally Enabled Science: CADENS 
Tools for Cinematic Sci Vis:
Our Open-Source Tool, ytini 


\section{yt + Houdini}

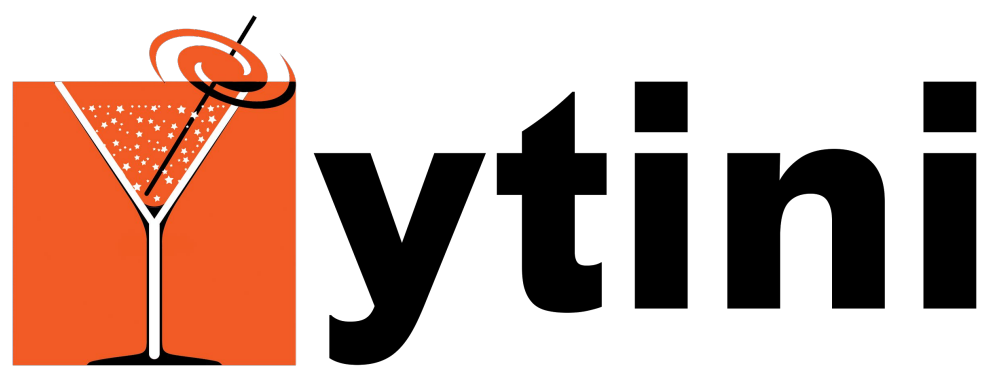

1. Use yt as a data reader

2. Write out data in OpenVDB format

3. Read data into Houdini

Yt is a great tool for easily reading and analyzing many complex types of scientific data, but Houdini provides the artistic flexibility that we need. To combine the best of both worlds, we created the middleware, ytini (rhymes with "martini"). In the simplest use case, we use yt to load the data, export it into the Houdini-compatible, sparse volumetric OpenVDB data format, and load the volume into Houdini.

Ytini is free and open-source. Visit http://ytini.com/ for setup instructions, Python code, tutorials, downloads, and more. Ytini is a collaboration between the AVL and Dr. Jill Naiman of the Harvard-Smithsonian Institute for Astrophysics. 


\section{AMR in Houdini with}

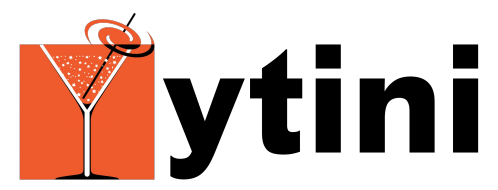



Rendering showing the underlying AMR structure

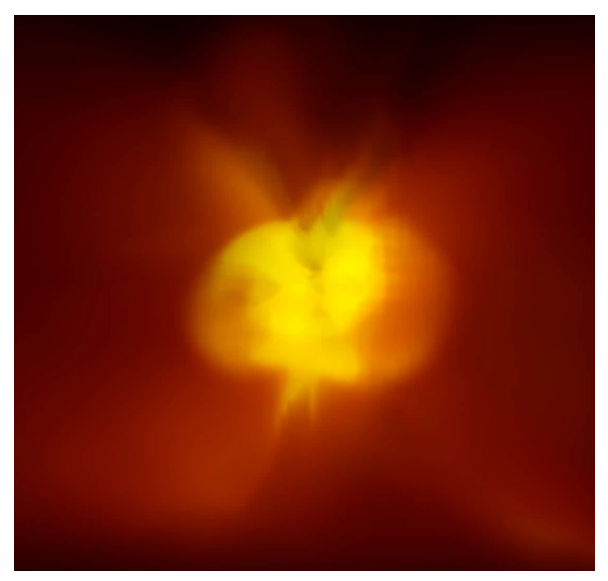

Final rendering with ytini

In a more complicated use case, even multi-resolution adaptive mesh refinement (AMR) data can be loaded into Houdini with ytini:

1. Use yt as a data reader.

2. Export each AMR level as a separate OpenVDB file, with two variables: a covering grid of the data extending once cell beyond the edge of the grid, and a cutout mask where the next level should be. (The extra cell information is needed for Houdini to correctly interpolate data values up to the boundaries of each cell.)

3. In Houdini, create a shader for each level that does the following:

a. Shifts each grid by $1 / 2$ voxel to compensate for cell-centered AMR vs. vertex-centered Houdini data representation.

b. Reads the mask by index to determine which VDB to render at any given sampling point. (Mantra/Houdini automatically interpolates volumes to create a smooth falloff at the edges. In this case, we do not want that interpolation; we want a clean cut so that the AMR boxes fit nicely within each other. Looking up the mask by index means that the mask value at the center of a cell applies to all rendering samples within that cell's boundary - mask values should not be interpolated.)

A pre-built shader is available for download at http://ytini.com/ , as well as a tutorial with step-by-step instructions. 


\section{Tools for Science:}

\section{Visual Molecular Dynamics, VMD}

Visual effects tools originally evolved from science and engineering tools, and early on they had significant overlap in functionality and design. Visual effects tools used by artists have since necessarily evolved in their own direction, which for years left an unnecessary gap between the rendering capabilities and interoperability of artistic tools and scientific visualization software. More recently, leading scientific visualization tools have begun to adopt some of the same state-of-the-art rendering technologies employed in visual effects. Many artists are unaware of the growing trend for scientific visualization tools to incorporate more advanced rendering technologies for built-in generation of production-level graphics, motivated by the combination of a desire for more easily interpreted images that communicate effectively by exploiting more of our visual intuition, and due to the rapidly growing size of scientific data beginning to make it impractical for scientists to use conventional outboard rendering tools.

John Stone is the lead software developer of a molecular visualization tool called "VMD", and he will describe the visualization and rendering capabilities of VMD as one example of how scientific visualization applications are being adapted to address the many challenges posed by large scientific data, and state-of-the-art simulations conducted on petascale supercomputers. 


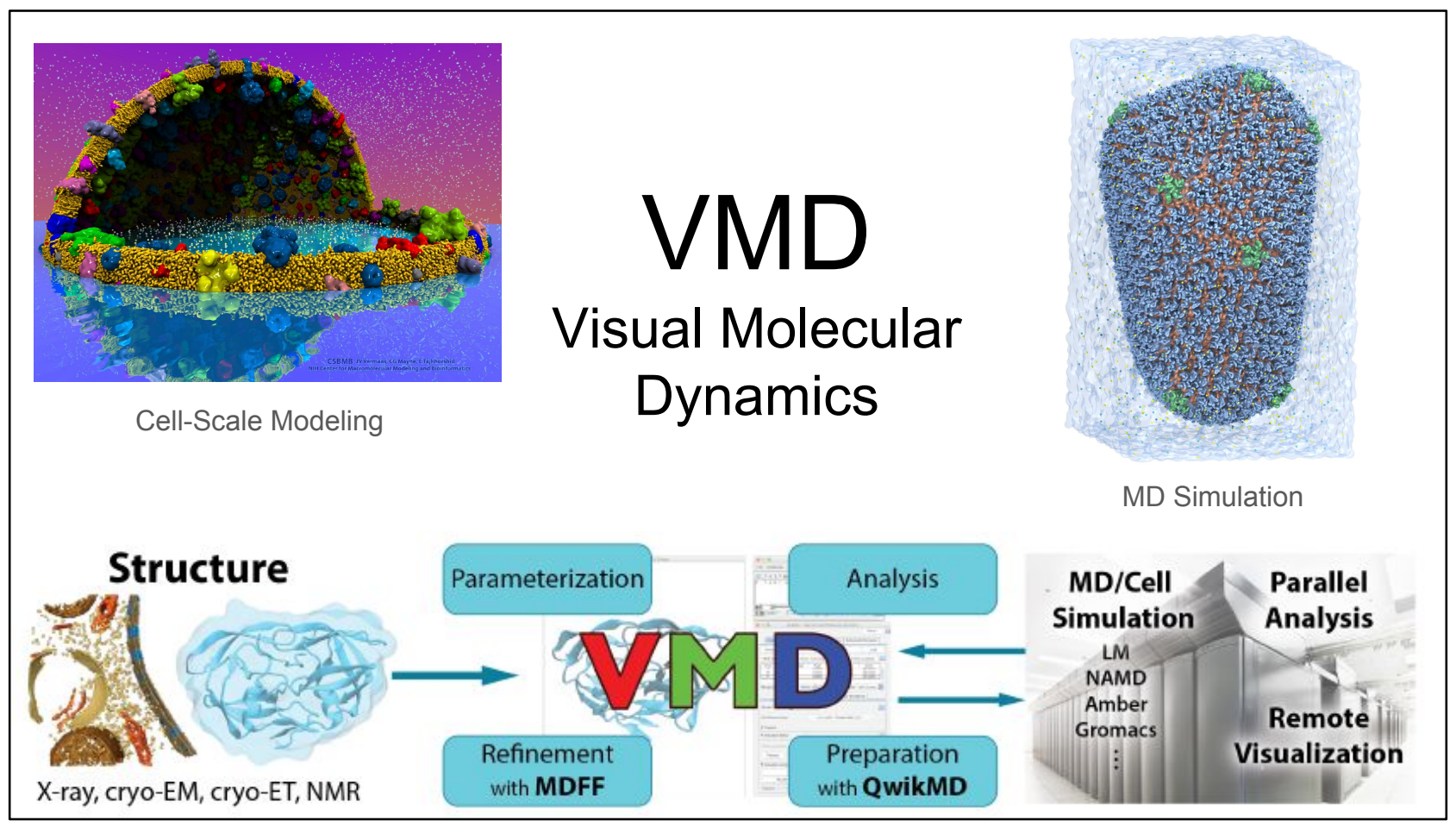

VMD is a biomolecular visualization tool that has been developed over more than 20 years, originally in immersive VR environments, and later for conventional desktop workstations. VMD is user extensible through scripting and plugins.

Visualization and analysis of:

- Molecular dynamics simulations

- $\quad$ Lattice cell simulations

- Quantum chemistry calculations

- $\quad$ Cryo-EM densities, volumetric data

- Sequence information

VMD is available at http://www.ks.uiuc.edu/Research/vmd/ 

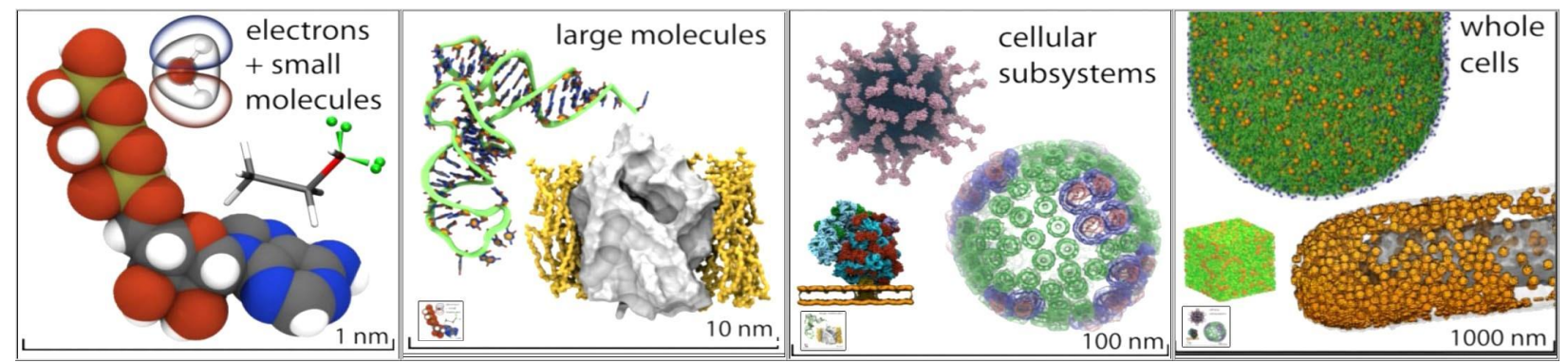

\section{System Size}

VMD serves many communities.

- VMD user statistics:

- $\quad 103,000$ unique registered users; 17,000 (16\%) are NIH funded researchers

- $\quad 20,000$ citations, over 2,800 citations per year

- $\quad$ Supports key data types, file formats, and databases

- User extensible to support new tools, data types, custom analyses 


\section{Goal: A Computational Microscope}

\section{Study the molecular machines in living cells}

Ribosome: target for antibiotics

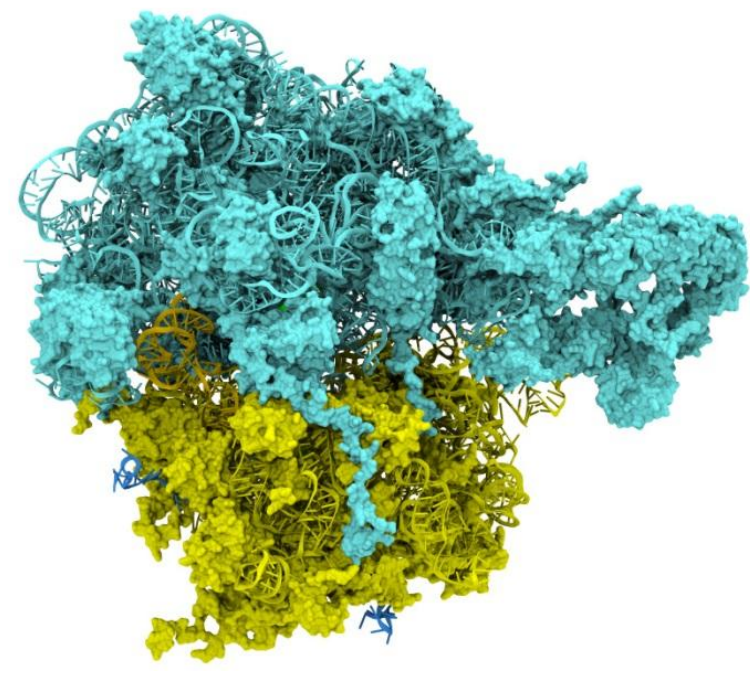

Poliovirus



Ribosomes synthesize new proteins from genetic information

Bacterial ribosome is a target for antibiotics, which interfere with its ability to function by binding to sites on the structure, preventing proteins from being synthesized

Ribosome simulations are extremely computationally challenging due to the size of the structure and its natural environment (solvent, ions, etc, large box, 3 million atoms or so)

Bionanodevice for sequencing DNA efficiently

Knowledge of an individual's genetic makeup can lead to the prediction and treatment of many diseases by the means of personal genomic medicine and pharmacogenomics. In order for genome sequencing to become practical for common medical use, an inexpensive high throughput genome sequencing technique is required. $\mathrm{NIH}$ has set a goal of reducing the cost of genome sequencing by a factor of 10,000 , to less than $\$ 1,000$ within ten years.

The computer is the best microscope to guide the development of bionanodevices, for example to sequence DNA. While experiments can measure DNA electrostatic potentials, they cannot visualize the DNA's location and conformation at the time of measurement. 


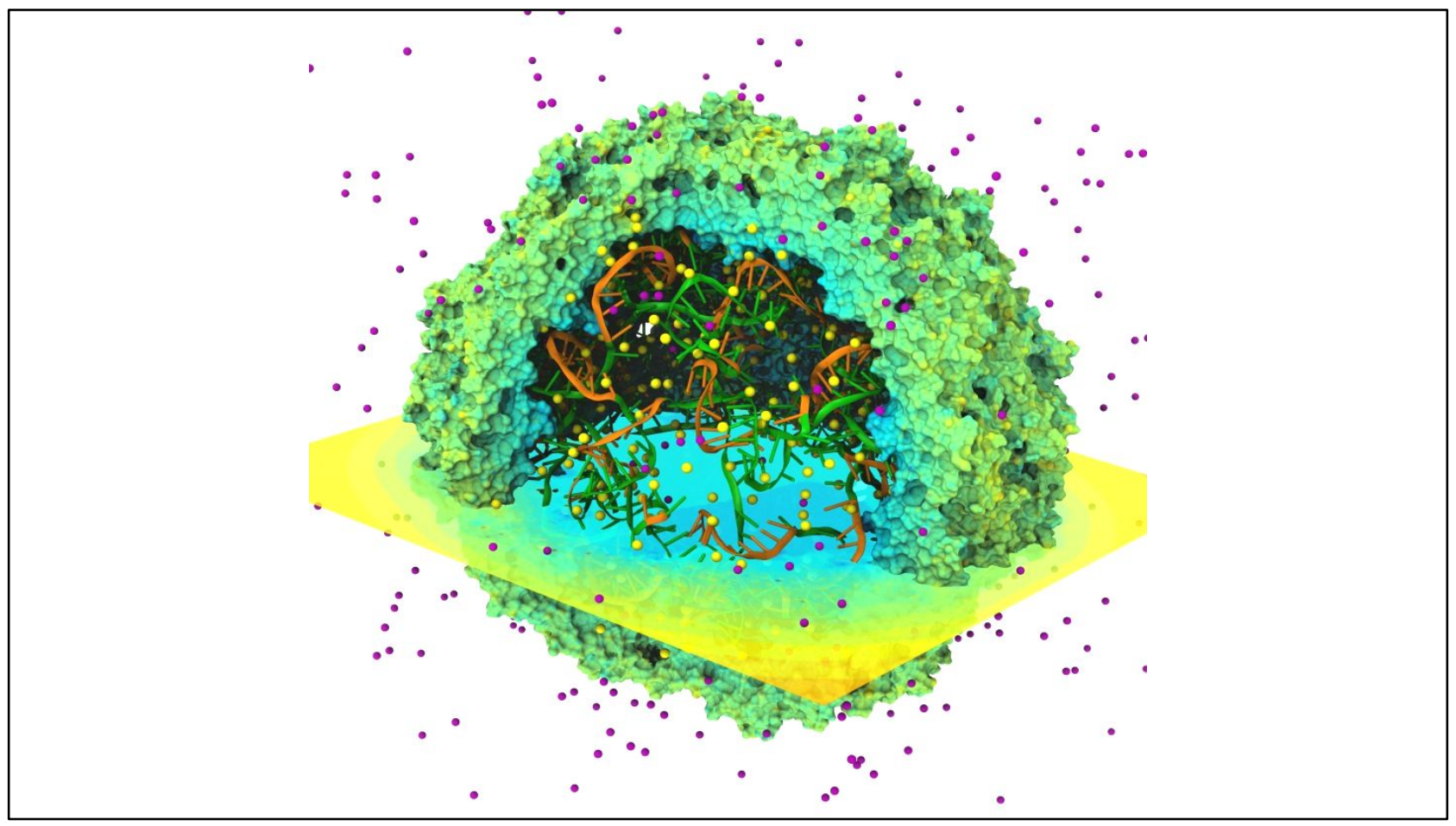

Biomolecular Visualization Challenges

- Geometrically complex scenes

- Spatial relationships important to see clearly: fog, shadows, AO helpful

- Often show a mix of structural and spatial properties

- Time varying!

Attributes of the Data We're Interested in Visualizing

- Multiple types of data

- Atomic structures, Sequences, Volumetric data, ...

- Many attributes per-atom

- Millions of atoms, particles, voxels

- Time varying (simulation trajectories)

- Multiple structures 




A major challenge faced by scientists that study biomolecular structures is the ongoing orders-of-magnitude increase in the size and complexity of atomic-detail structures available from experimental imaging and hybrid modeling approaches. The rapid growth in the size of the systems being studied presents a formidable challenge for all tasks involved in modeling, simulating, analyzing, and visualizing these health-relevant structures and their dynamics. Effective visualization of these biomolecular systems involves a lot of creativity, and often requires compromises between interactivity, visual fidelity, and data management challenges of various kinds. 




Visualizing Data with Shape

- Direct rendering of geometry from physical data, e.g., atomic structures

- Indirect rendering of data, feature extraction, e.g., density isosurfaces

- Reduced detail representations of data, e.g., protein ribbons, "cartoon"

- Use of size for emphasis 


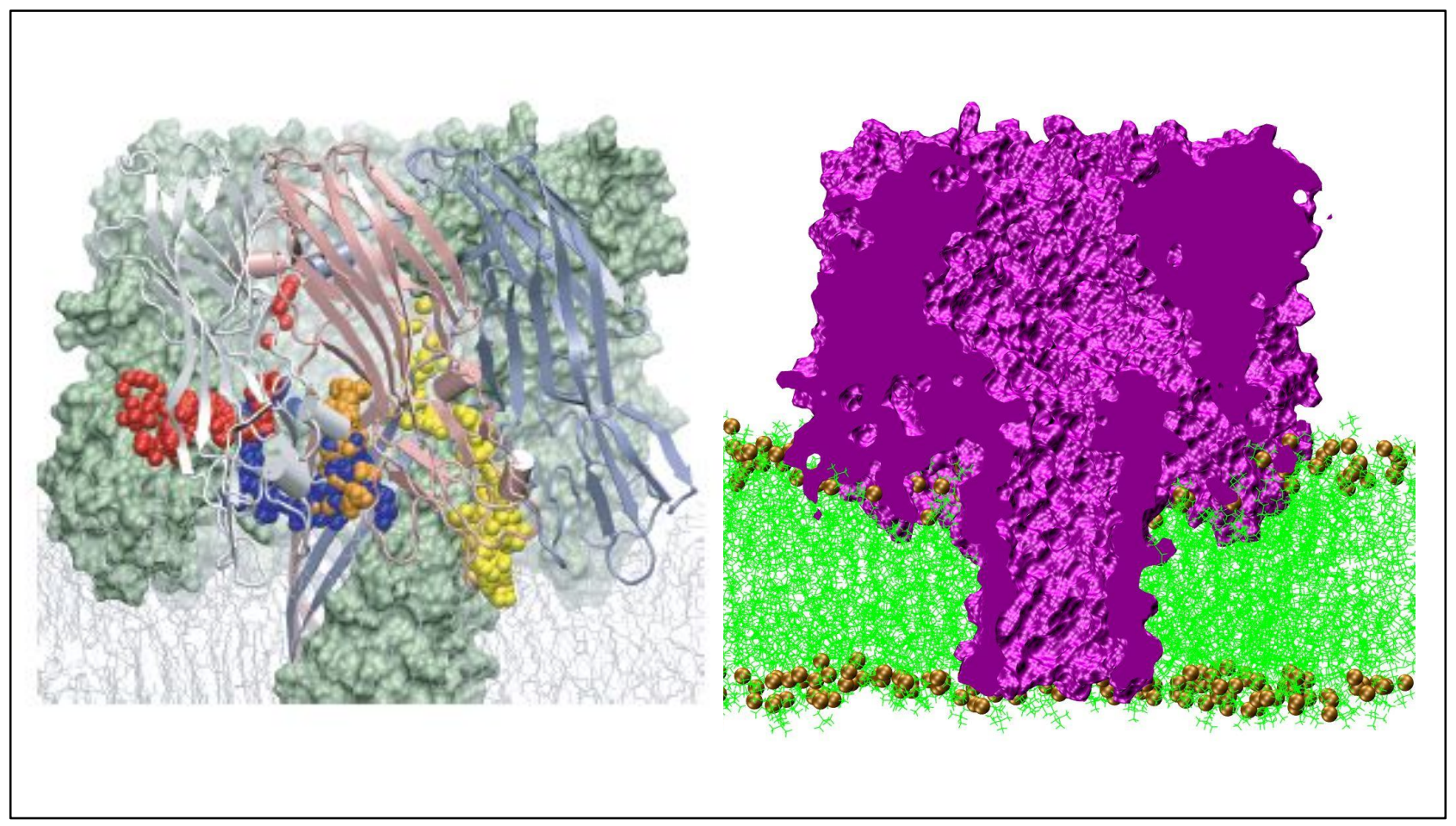

Multiple Representations, Cut-away Views

- Multiple representations are often used concurrently

- Show selected regions in full atomic detail

- Simplified cartoon-like or schematic form

- $\quad$ Clipping planes can slice away structure obscuring interesting features 


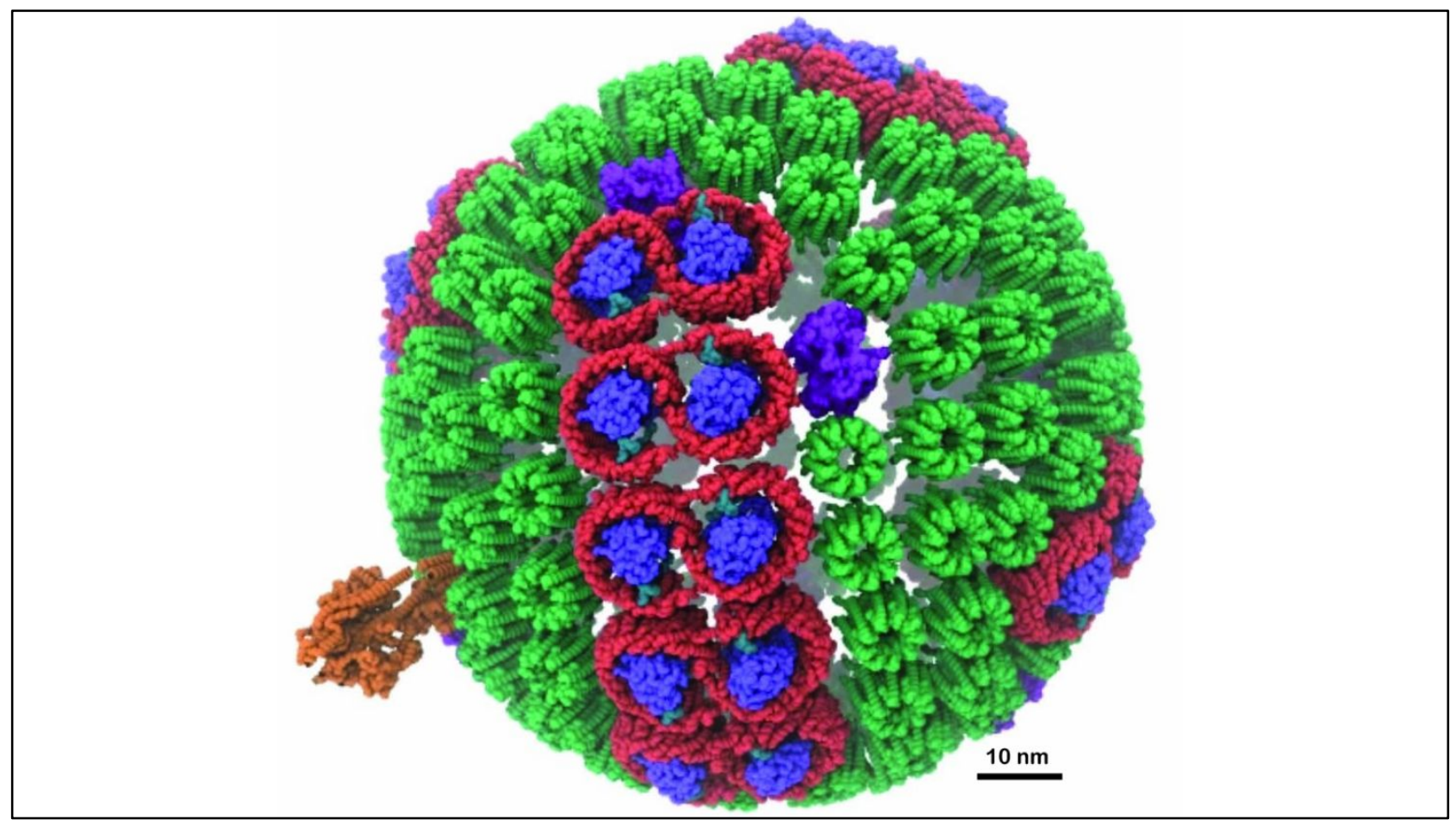

Photosynthetic Chromatophore of Purple Bacteria

- Purple bacteria live in light-starved conditions at the bottom of ponds, with $\sim 1 \%$ sunlight

- Chromatophore system

- $100 \mathrm{M}$ atoms, $700 \AA^{3}$ volume

- Contains over 100 proteins, $~ 3,000$ bacteriochlorophylls for collection of photons

- Energy conversion process synthesizes ATP, which fuels cells... 


\section{VMD Representation Examples}

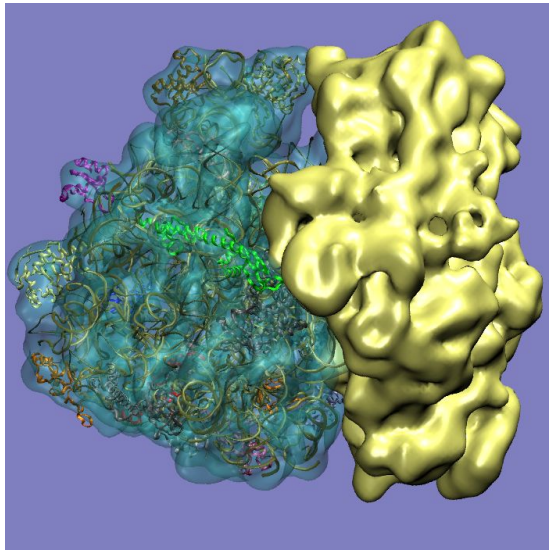

Ribosome, J. Frank

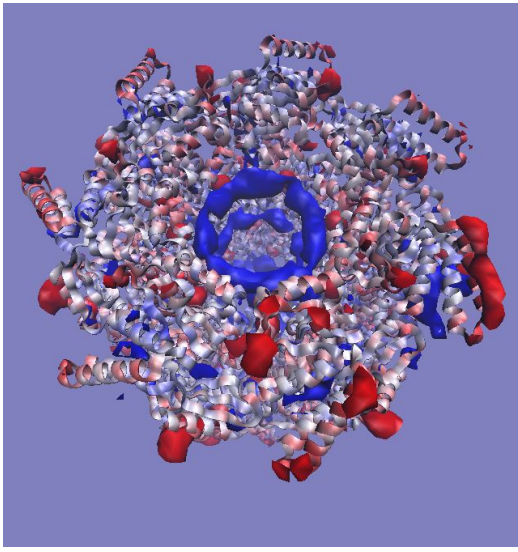

GroEL /w Situs

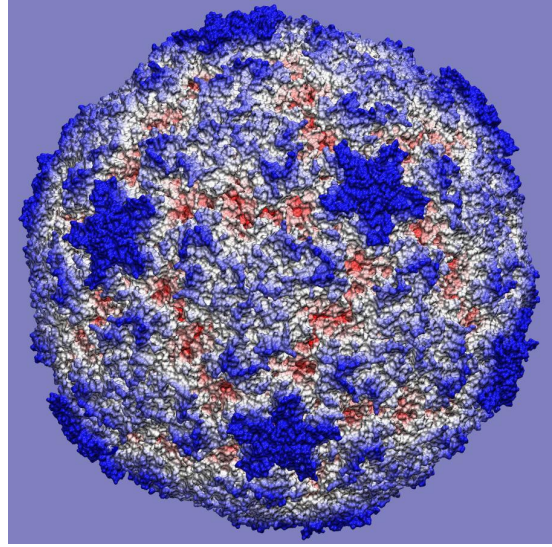

4HRV, 400K atoms

- Draw atomic structure, protein backbone, secondary structure, solvent-accessible surface, window-averaged trajectory positions, isosurfaces of volumetric data, much more...

- Color by per-atom or per-residue info, position, time, electrostatic potential, density, user-defined properties, etc... 

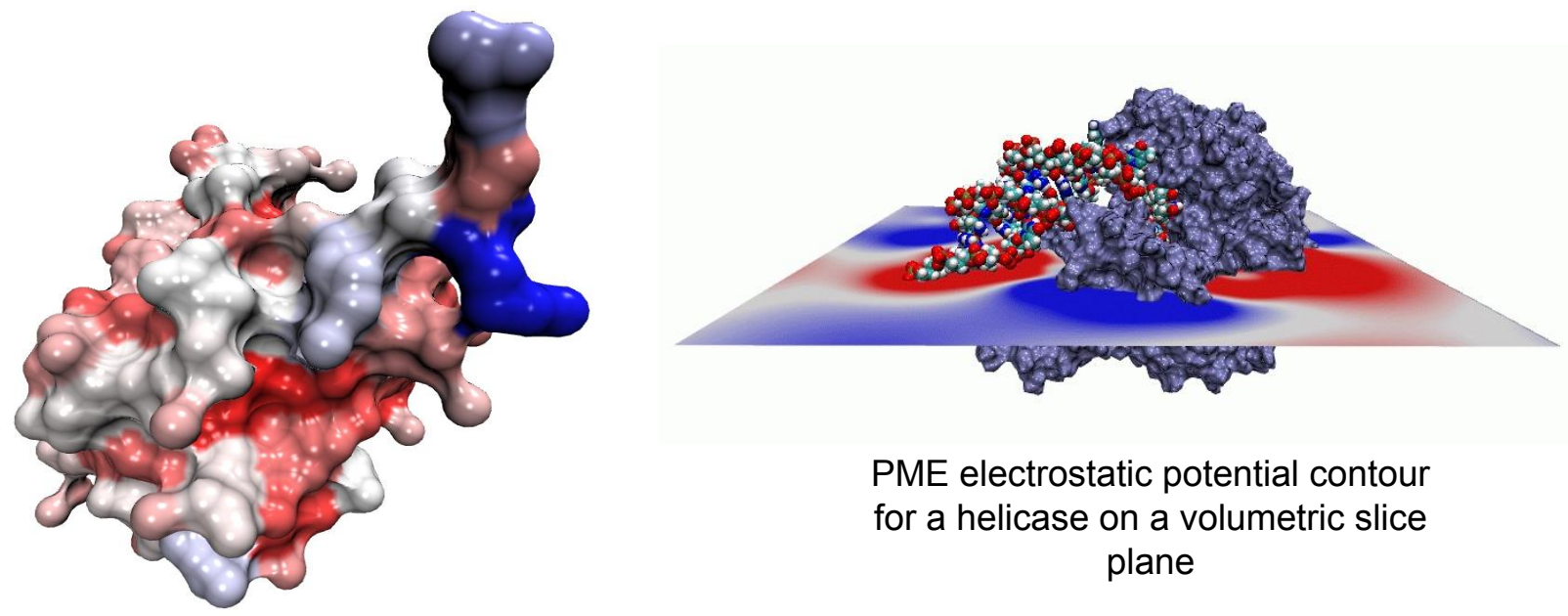

PME electrostatic potential contour for a helicase on a volumetric slice plane

Per-residue solvent-accessible surface area of Ubiquitin

Visualizing Data with Texture and Color

- Direct mapping of properties/values to colors (e.g. color by electrostatic potential)

- Indirect mapping via feature extraction (e.g. color by secondary structure)

- Use saturated colors to draw attention

- Use faded colors and transparency to de-emphasize

- Use depth cueing/fog to improve depth perception or to de-emphasize background environment

\section{Computed Properties}

- Smoothing of thermal noise

- Secondary structure

- Hydrogen bonds, salt bridges

- Forces, energies, stress, strain

- Time averaging of electrostatic fields, occupancy maps

- Quality-of-fit cross correlation with cryo-EM density maps

- Normal modes, principal component analysis, essential dynamics

- Cluster simulation trajectory timesteps by structural similarity 


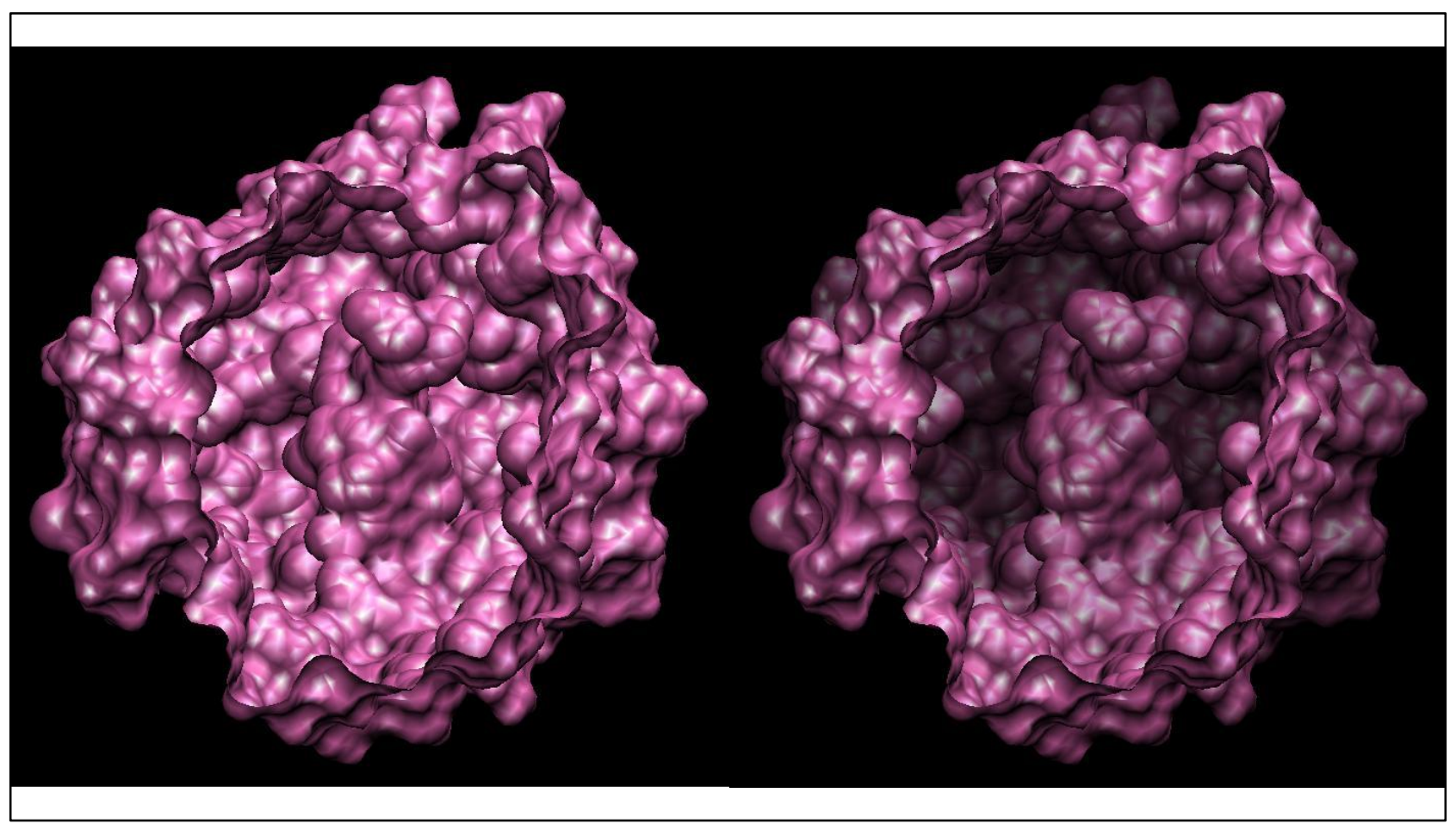

Demonstration of OpenGL and Depth Cueing 


\section{Selection, Filtering}

- Most viz tools allow interactive visual picking, menu-driven selections of structure components to display or operate on

- VMD also extensively uses a text-based selection language (think google):

- "water within 10 of protein and $z>0$ "

- Allows selection on user-defined fields

- Promotes synergy between interactive and scripting interfaces

- Works very well when dealing with huge time-varying structures

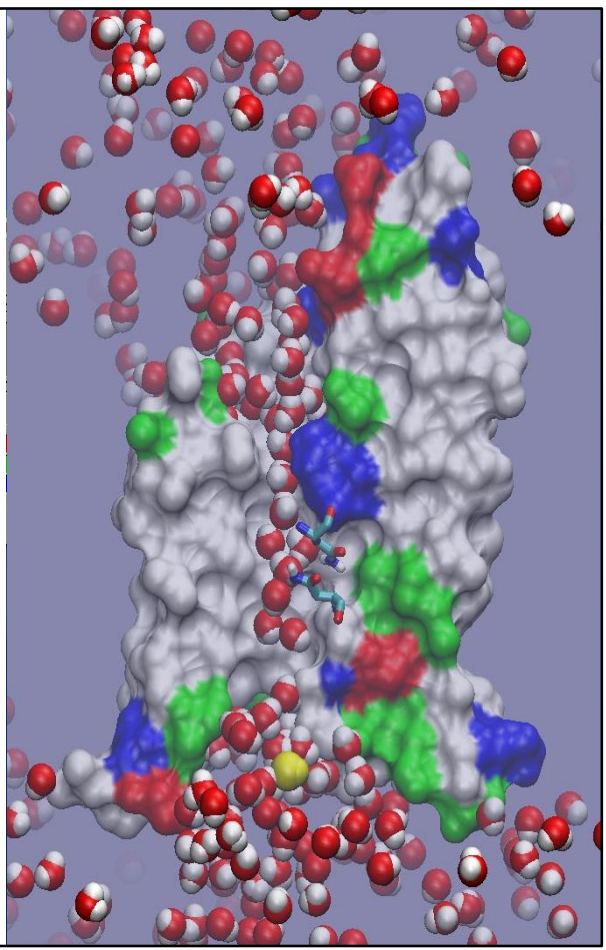

One concern that arises when making use of visualizations originally designed for use by domain scientists in the context of cinematic visualizations that target a much broader audience involves the ways in which scientists frequently eliminate information that is either distracting or uninteresting in the context of the scientific study they are conducting. A domain scientist with expertise in the field often knows what assumptions are being made and what structural information is not shown for the benefit of clarity of scientific communication, but a lay audience does not.

To make existing material useful in cinematic visualizations that target a broader audience, care must be taken to re-add many pieces of missing context that a domain expert knows and/or assumes but that would unintentionally mislead a non-specialist audience unfamiliar with common conventions in the field. In the image shown above, the water molecules that are not involved in interacting with the aquaporin channel are excluded from the visualization, and half of the channel structure has been cut away to reveal the interior. A domain expert looking at this image would know what structure has been deselected for the purposes of improved visual focus and clarity, but a lay audience would not know what is "missing" from the picture or its surroundings. This is a key concern when developing cinematic visualizations from existing scientific materials. 


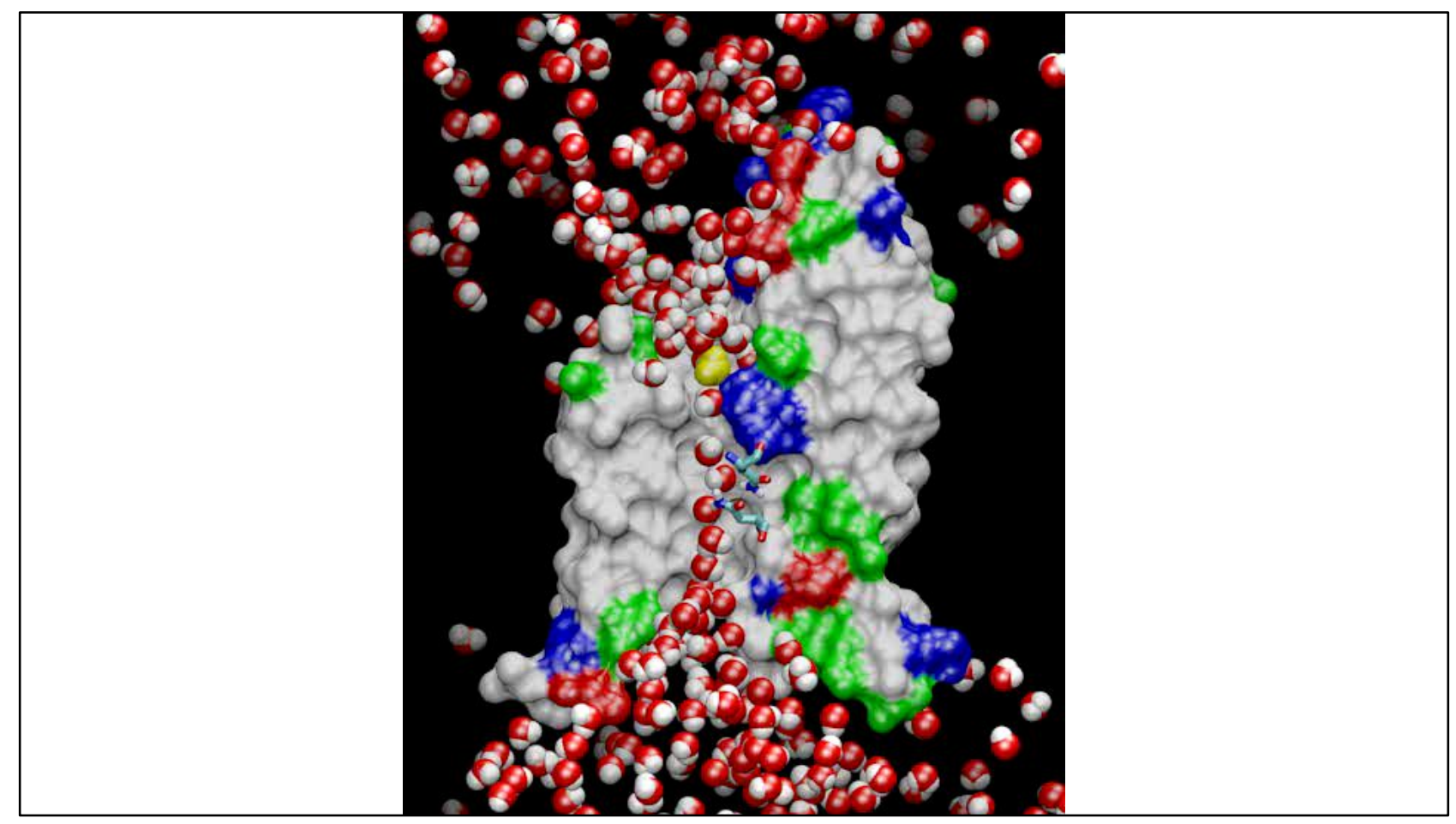

Trajectory Animation

- Motion aids perception of shape, understanding of dynamic processes

- Animate entire model, or just the parts where motion provides insight

- Window-average positions on-the-fly to focus on significant motions

- Selected atoms updated on-the-fly (distance constraints, etc) 


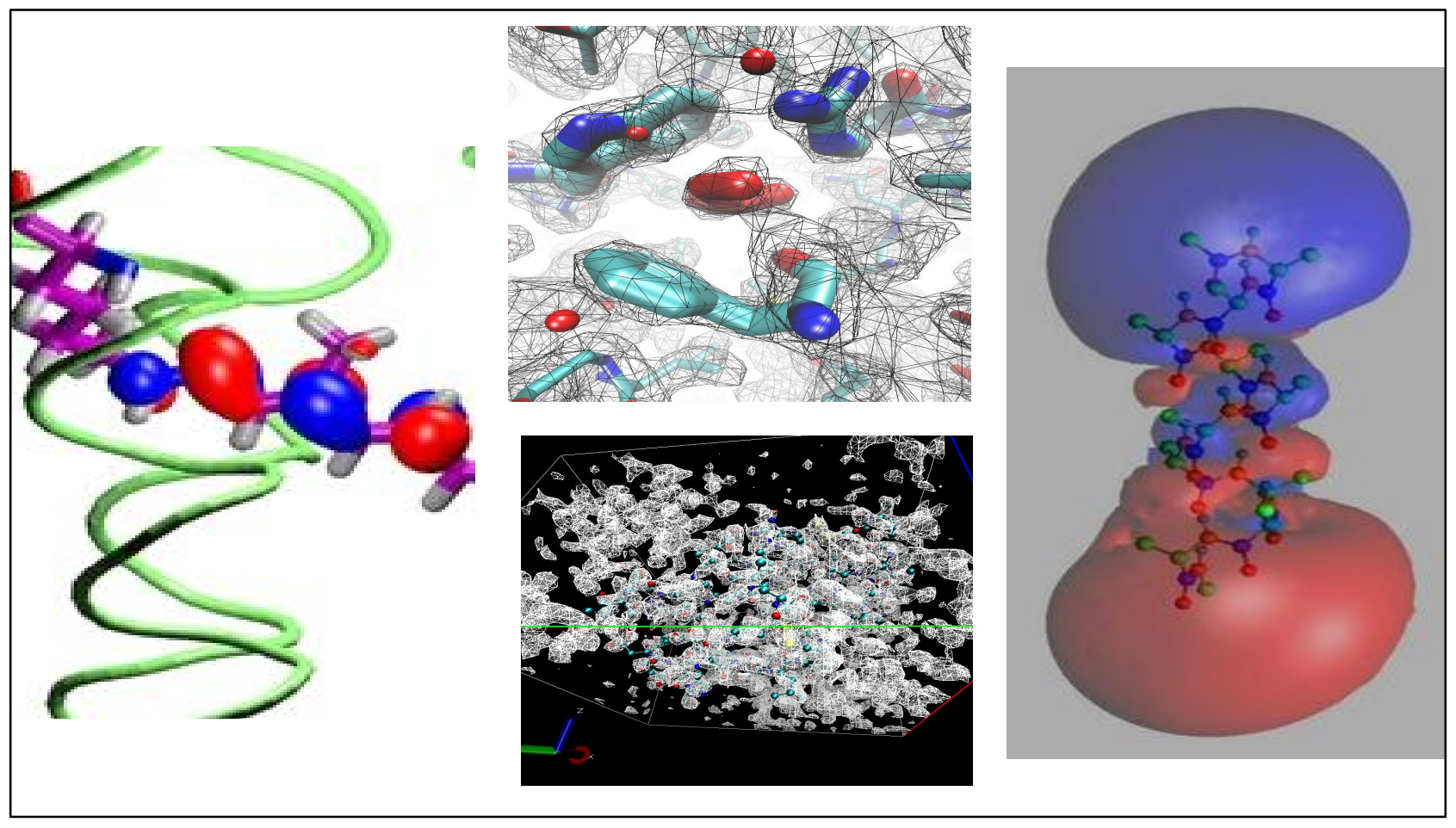

Visualizing Volumetric Data

- Display environment surrounding molecular structure, fields that affect structure and function

- Electron orbitals, electron density, electrostatic potential, temporal occupancy maps 




73 


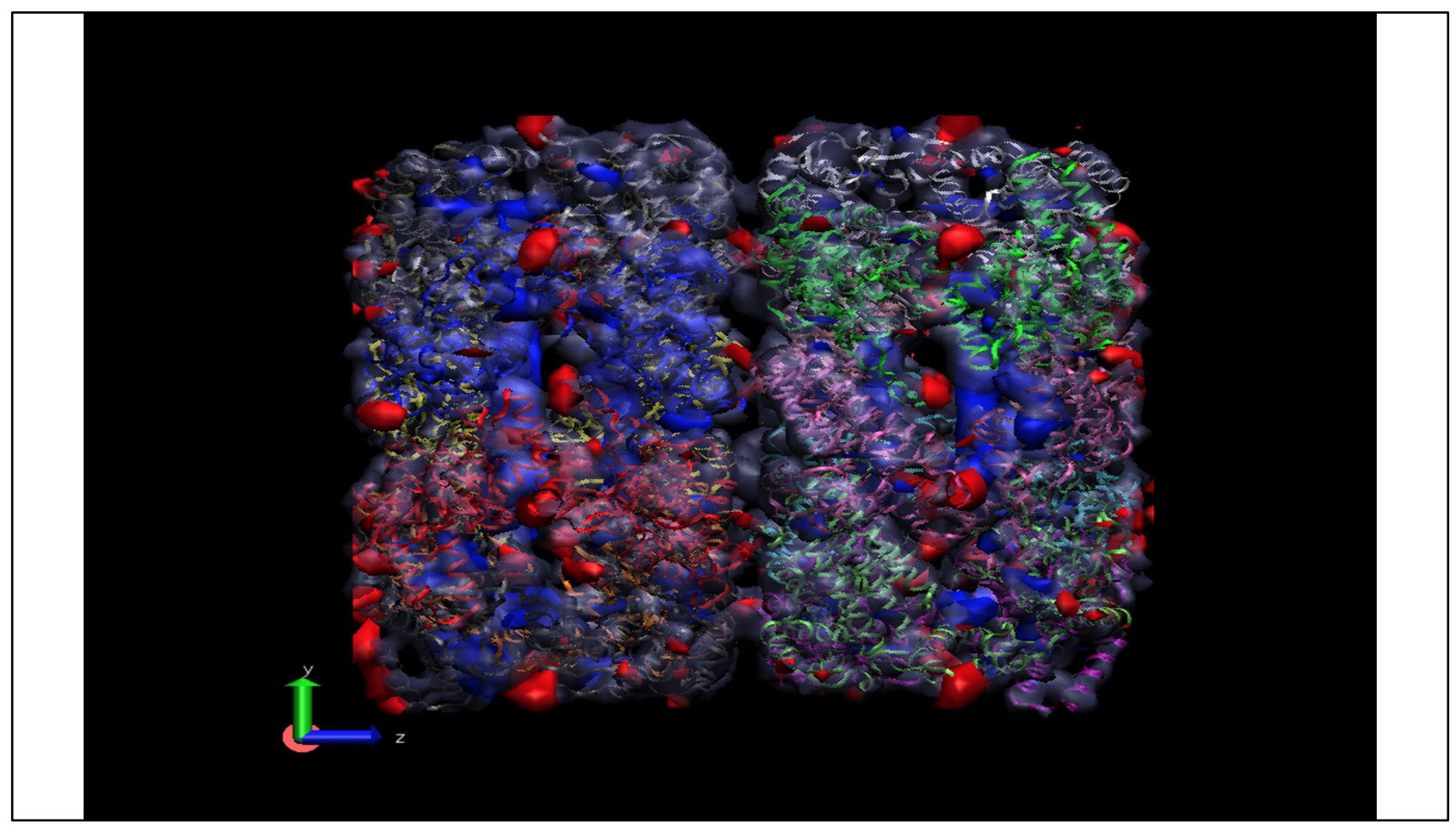

GroEL: Docked Map and Structure

Dock cryo-EM density map with all-atom structure

- Compute simulated density map from all-atom structure

- Calculate difference between cryo-EM density map and simulated map

- Display isosurfaces

- Display map/structure alignment error as isosurfaces

- Texture by density or map/structure alignment error 


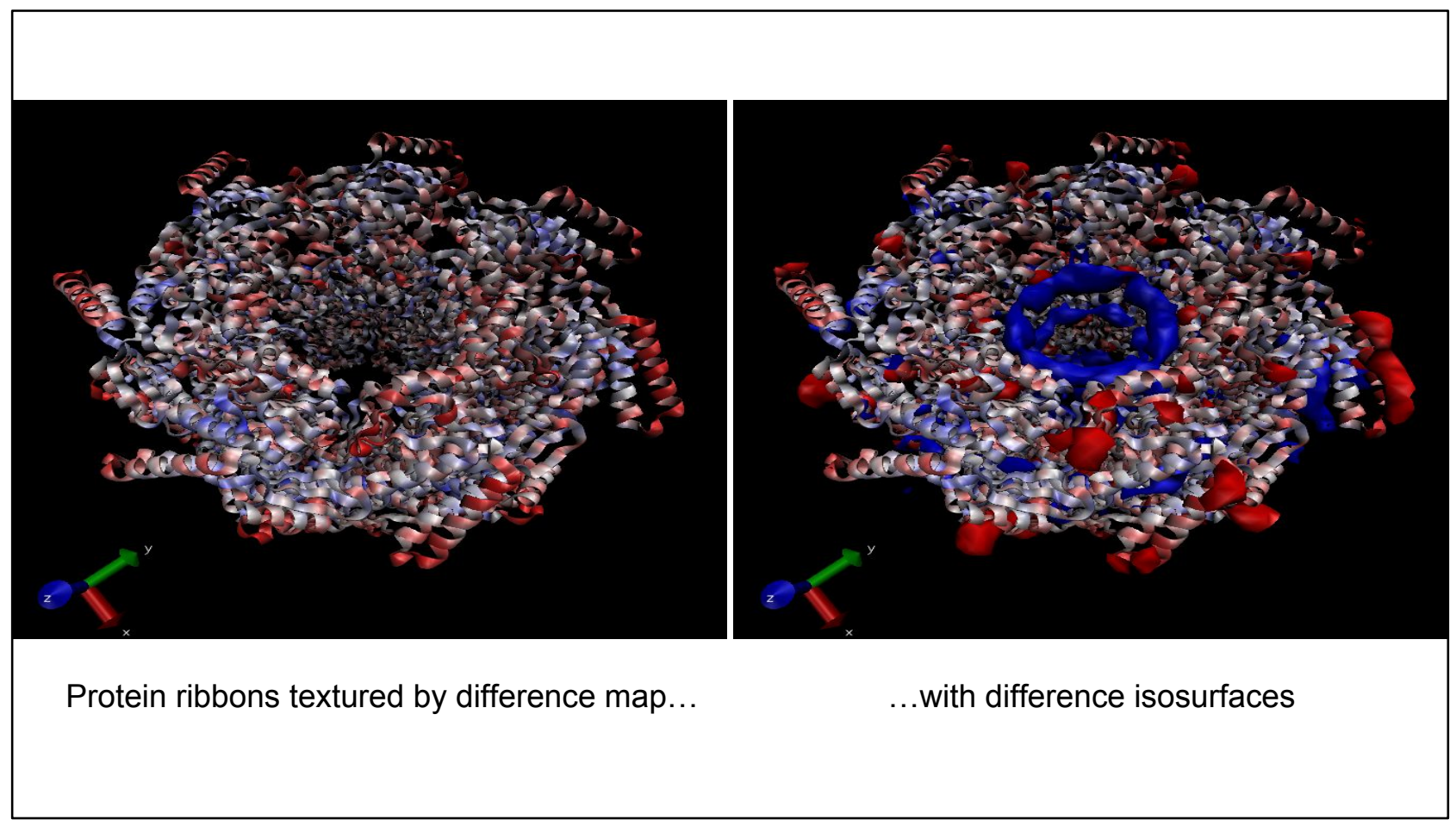

GroEL: Display of Difference, Error 


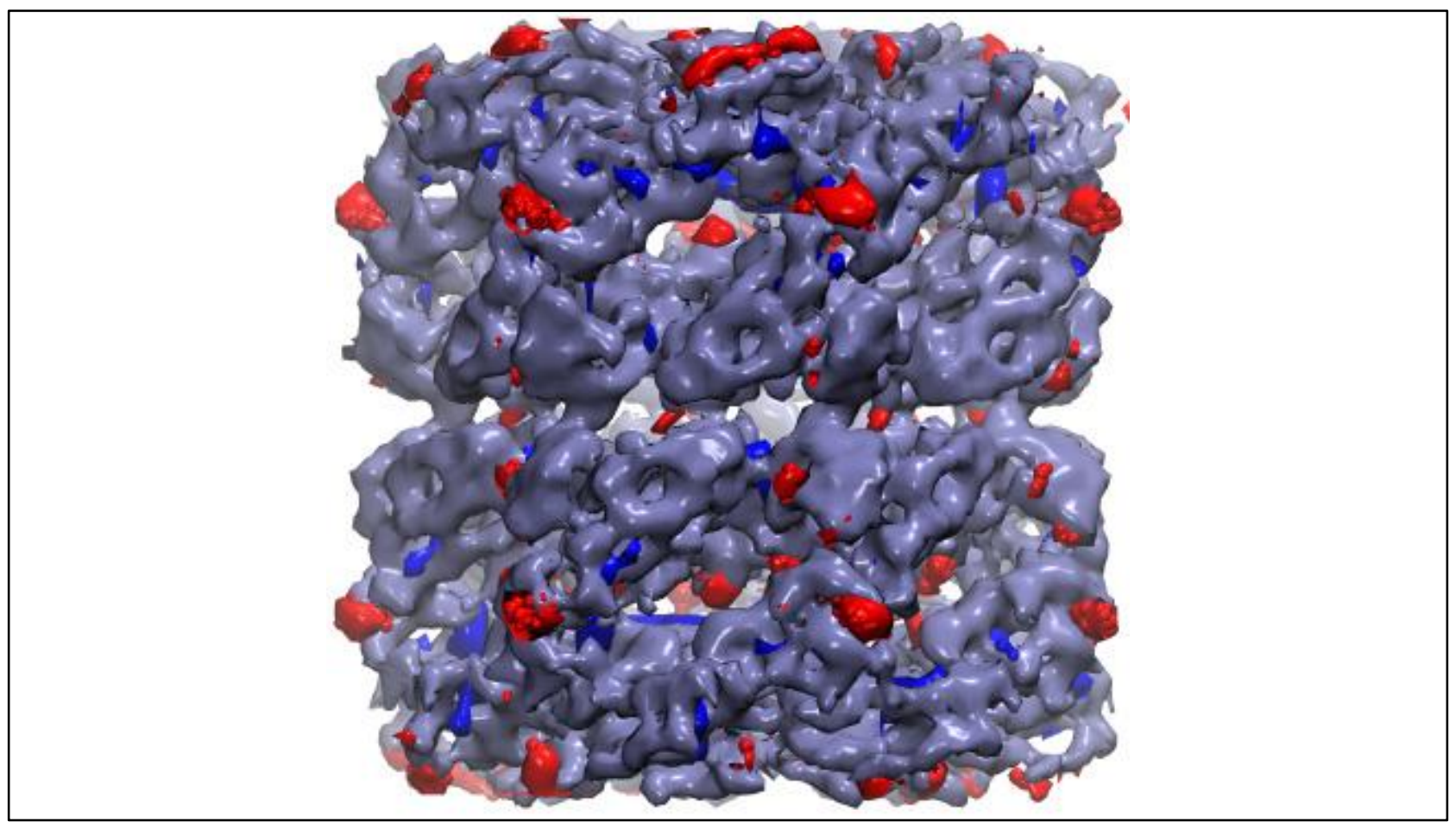

GroEL: Density Difference Isosurfaces 



Visualizing Data Topologically

- Data relationships indicated by grouping (e.g. phylogenetic trees)

- Abstract or schematic representation, e.g. Ramachandran plot 


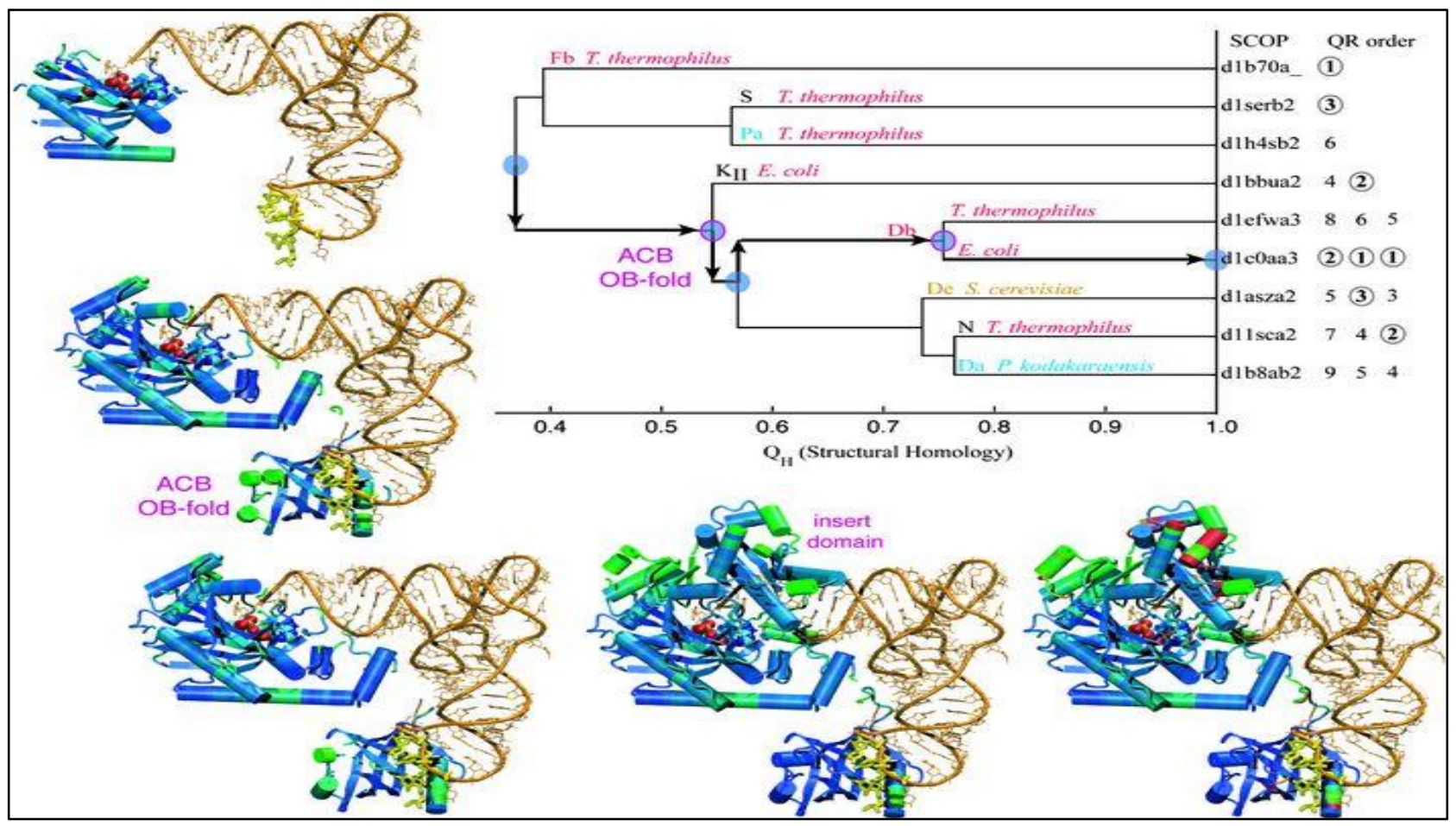

Multiple Structure Alignment

- Study evolutionary changes in sequence and structure of proteins

- Align and superimpose multiple structures

- Color by structural conservation

- Color by sequence conservation

- Display phylogenetic tree, cluster biological form by similarity 

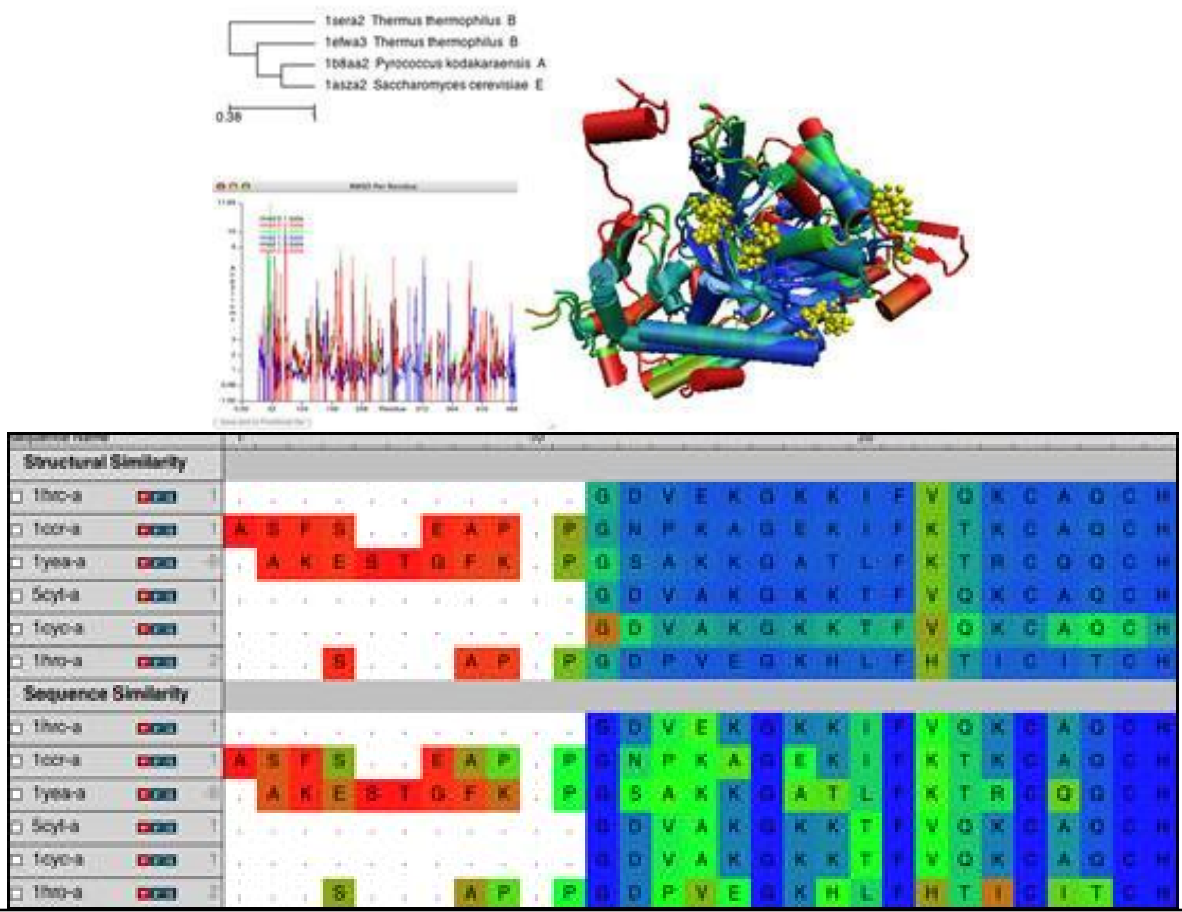

Multiple Data Modalities

- Aligned sequences and structures, phylogeny

- Simultaneous use of shape, color, topology, and interactivity

- Multiple simultaneous representations

- Multiple data display modalities

- Selections in one modality can be used to highlight or select in others 


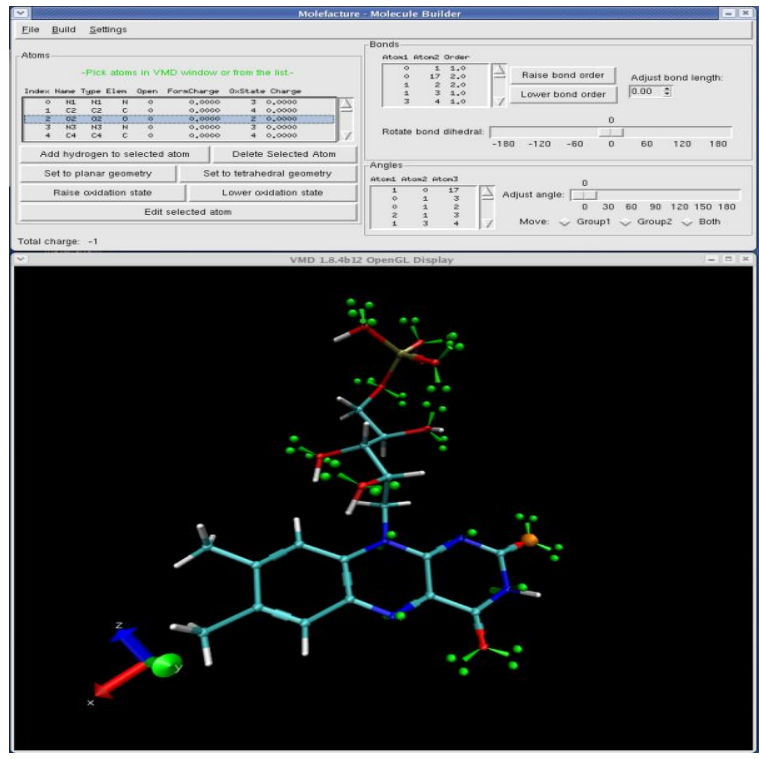

Molefacture Plugin

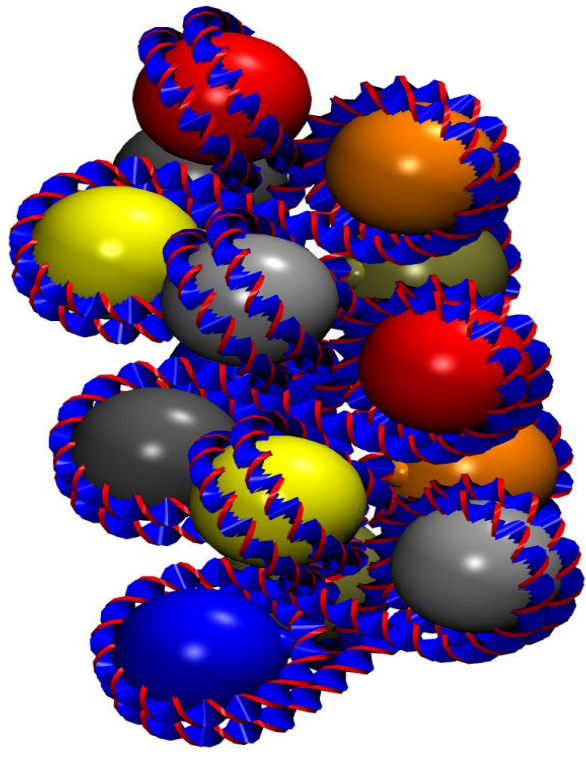

"VDNA" Chromatin Rendering Script

Extensibility - VMD Adaptable by Researchers, Community Developed Tools 

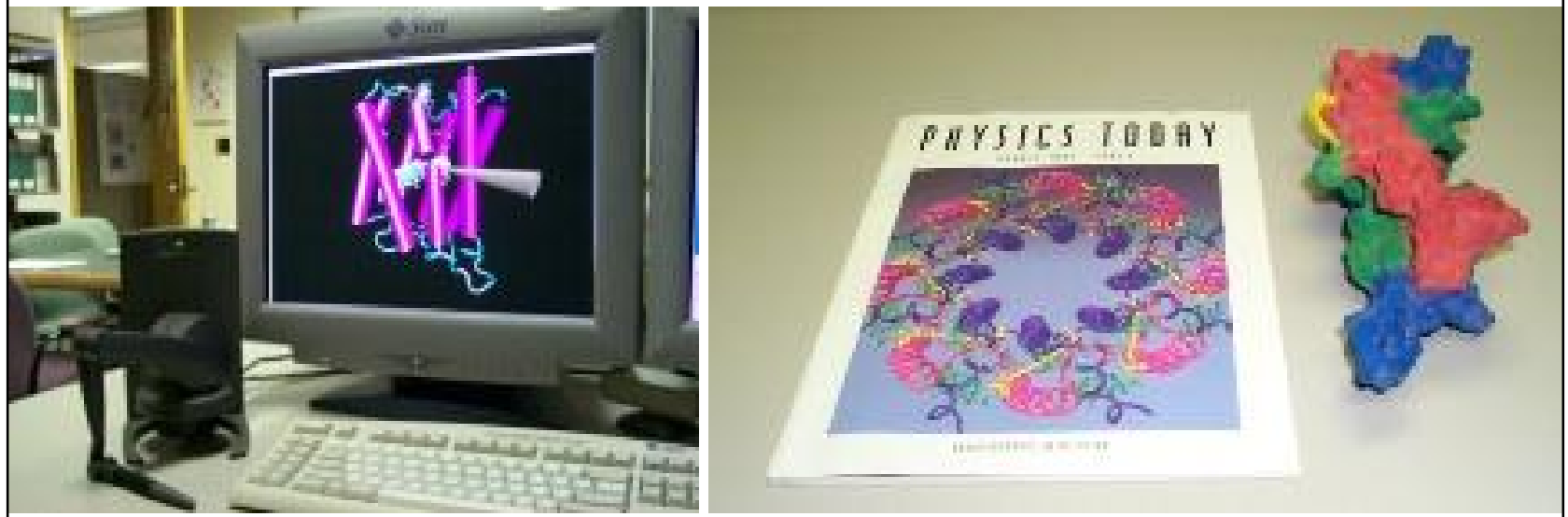

What Else Can We do?

Enhance visual perception of shape

- Motion, interactive rotation

- Stereoscopic display

- High quality surface shading and lighting

Enhance tactile perception of shape

- Print 3-D solid models

- Interactive exploration using haptic feedback 




Schematic Representations

- Extract and render pores, cavities, indentations

- Simplified representations of large structural features 


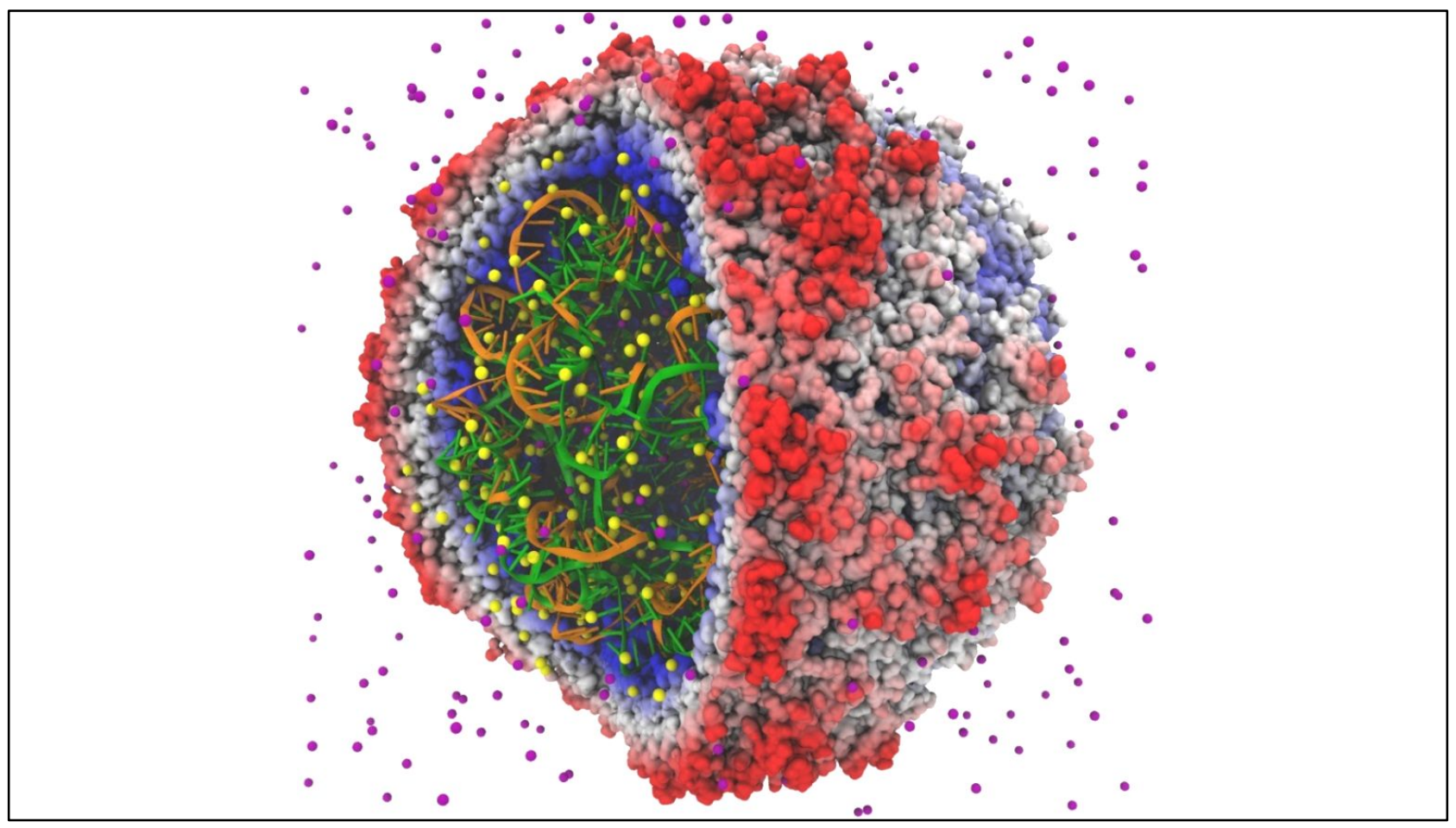

VMD “QuickSurf” Representation of Satellite Tobacco Mosaic Virus

- Displays continuum of structural detail:

- All-atom, coarse-grained, cellular models

- Smoothly variable detail controls

- $\quad$ Linear-time algorithm, scales to millions of particles, as limited by memory capacity

- Uses multi-core CPUs and GPU acceleration to enable smooth interactive animation of molecular dynamics trajectories $w /$ up to $\sim 2$ million atoms

Image credits:

Fast Visualization of Gaussian Density Surfaces for Molecular Dynamics and Particle System Trajectories.

M. Krone, J. E. Stone, T. Ertl, K. Schulten. EuroVis Short Papers, pp. 67-71, 2012 


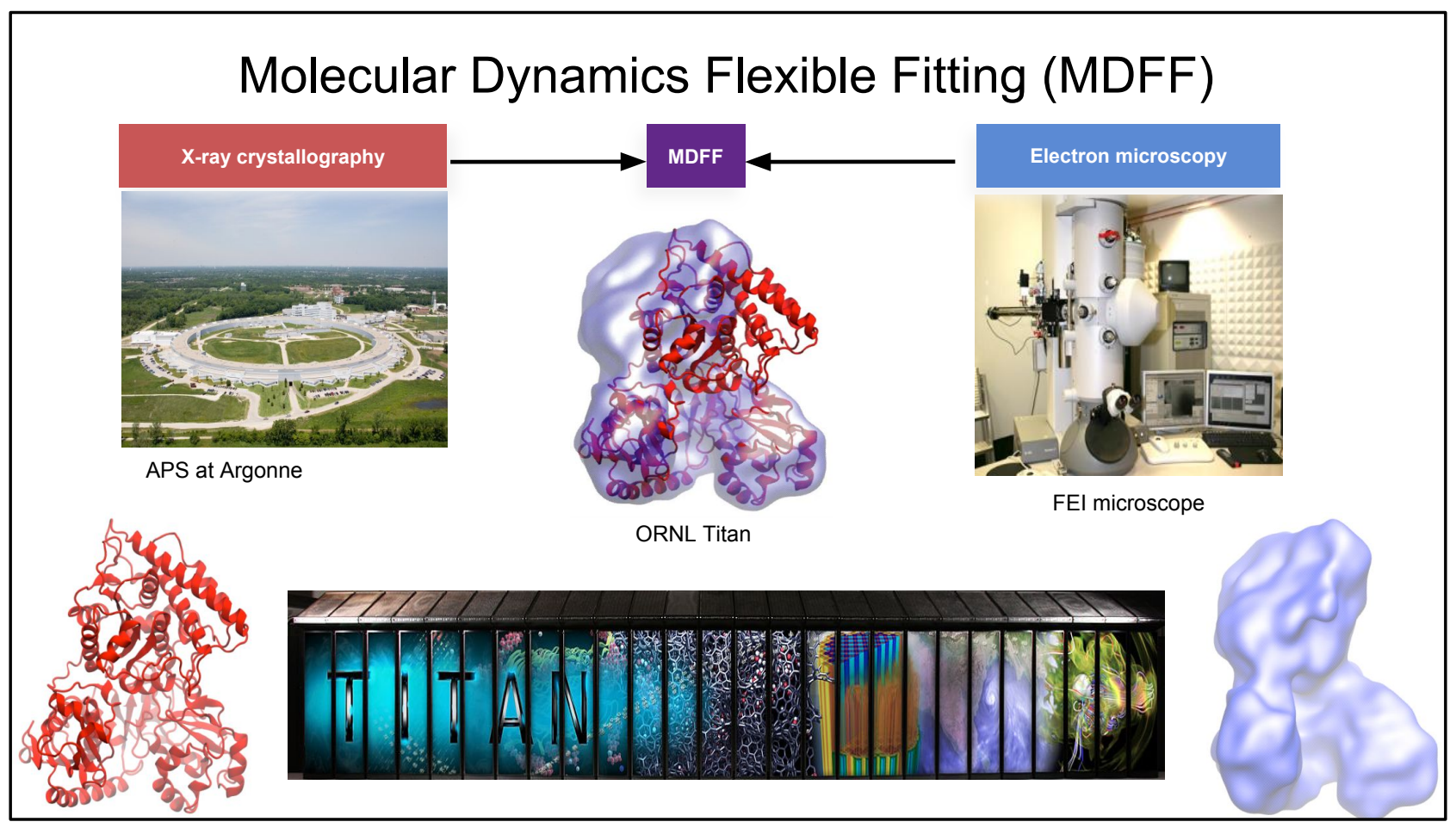

Flexible fitting of atomic structures into electron microscopy maps using molecular dynamics.

Image credits:

L. Trabuco, E. Villa, K. Mitra, J. Frank, and K. Schulten. Structure, 16:673-683, 2008. 


\section{VMD Timeline Plugin: Analyze MD Trajectories for Events}

\section{GPU-accelerated MDFF Cross Correlation Timeline}

Regions with poor fit Regions with good fit
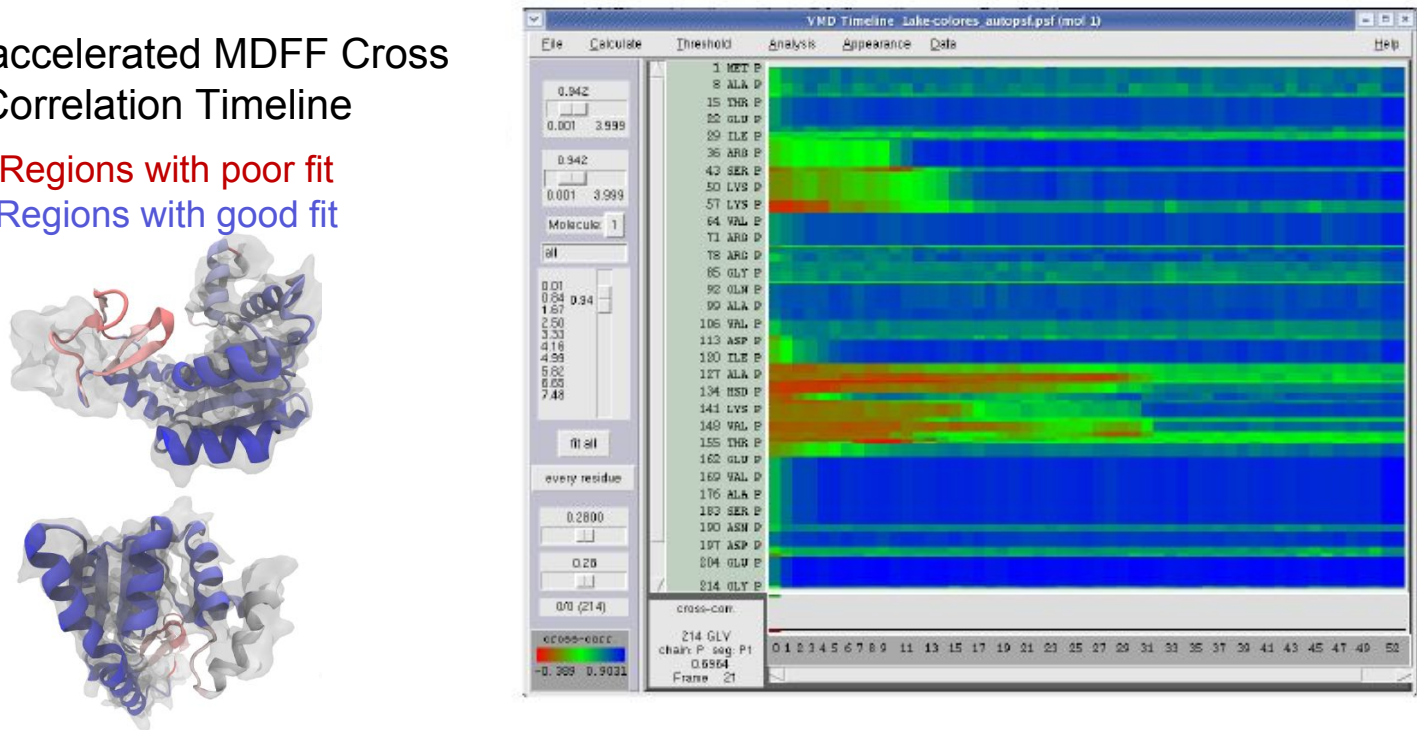

-Quality-of-fit between cryo-EM density map vs. all-atom molecular structure -Interactive 2-D heatmap plot linked to time-varying 3-D atomic structure - Single picture shows changing properties across structure+trajectory -Explore time vs. per-selection attribute, linked to molecular structure -Many analysis methods available; user-extendable 
- Build spatial acceleration data structures, optimize data for GPU

- Compute 3-D density map:

$$
\rho\left(\vec{r} ; \vec{r}_{1}, \vec{r}_{2}, \ldots, \vec{r}_{N}\right)=\sum_{i=1}^{N} e^{\frac{-\left|\vec{r}-\vec{r}_{i}\right|^{2}}{2 \alpha^{2}}}
$$

- Truncated Gaussian and spatial acceleration grid ensure linear time-complexity

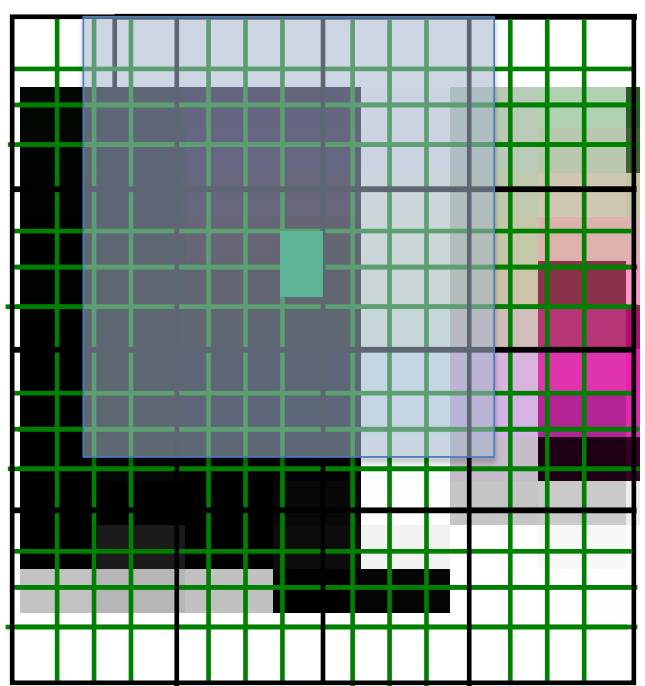

3-D density map lattice point and the neighboring spatial acceleration cells it references

Molecular Dynamics Flexible Fitting (MDFF) Density Map Algorithm 


\section{Single-Pass MDFF GPU Cross-Correlation}

3-D density map decomposes into 3-D grid of $8 \times 8 \times 8$ tiles containing $\mathrm{CC}$ partial sums and local CC values

Small 8x8x2 CUDA thread blocks afford large per-thread register count, shared memory Each thread computes
z-axis density map
lattice points and
associated CC partial
sums

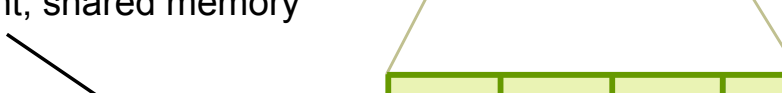

Fusion of density and CC calculations into a single CUDA kernel!!!

Spatial CC map and overall $\mathrm{CC}$ value computed in a single pass

Padding optimizes global memory performance, guaranteeing coalesced global memory accesses

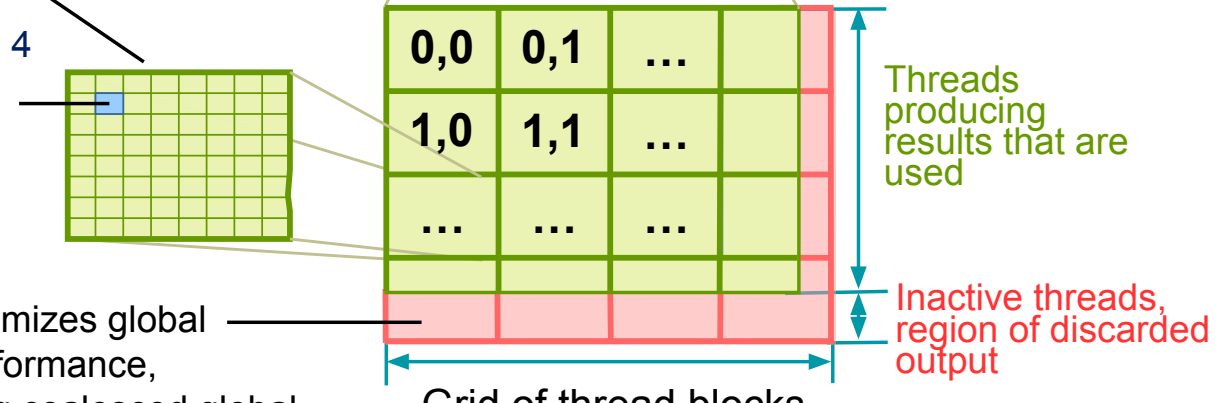

Grid of thread blocks

Threads in a thread block (potentially) execute in parallel

Threads in a block can communicate via fast shared memory Global memory reads are slow

Constant memory is fast, but can only be written by host at kernel startup. 


\section{VMD GPU Cross Correlation Performance}

\begin{tabular}{|c|c|c|c|c|}
\hline & RHDV & $\begin{array}{l}\text { Mm-cpn } \\
\text { open }\end{array}$ & GroEL & Aquaporin \\
\hline Resolution (A) & 6.5 & 8 & 4 & 3 \\
\hline Atoms & $702 K$ & $61 K$ & $54 K$ & $1.6 \mathrm{~K}$ \\
\hline $\begin{array}{l}\text { VMD-CUDA } \\
\text { Quadro K6000 }\end{array}$ & $\begin{array}{l}0.458 \mathrm{~s} \\
34.6 \mathrm{x}\end{array}$ & $\begin{array}{l}0.06 s \\
25.7 x\end{array}$ & $\begin{array}{l}0.034 \mathrm{~s} \\
36.8 \mathrm{x}\end{array}$ & $\begin{array}{l}0.007 s \\
55.7 x\end{array}$ \\
\hline $\begin{array}{l}\text { VMD-CPU-SSE } \\
\text { 32-threads, 2x Xeon E5-2687W }\end{array}$ & $\begin{array}{l}0.779 s \\
20.3 x\end{array}$ & $\begin{array}{l}0.085 \mathrm{~s} \\
18.1 \mathrm{x}\end{array}$ & $\begin{array}{l}0.159 \mathrm{~s} \\
7.9 \mathrm{x}\end{array}$ & $\begin{array}{l}0.033 \mathrm{~s} \\
11.8 \mathrm{x}\end{array}$ \\
\hline $\begin{array}{l}\text { Chimera } \\
\text { 1-thread Xeon E5-2687W }\end{array}$ & $\begin{array}{l}15.86 \mathrm{~s} \\
1.0 \mathrm{x}\end{array}$ & $\begin{array}{l}1.54 \mathrm{~s} \\
1.0 \mathrm{x}\end{array}$ & $\begin{array}{l}1.25 \mathrm{~s} \\
1.0 \mathrm{x}\end{array}$ & $\begin{array}{l}0.39 \mathrm{~s} \\
1.0 \mathrm{x}\end{array}$ \\
\hline
\end{tabular}

Image credit:

GPU-Accelerated Analysis and Visualization of Large Structures Solved by Molecular Dynamics Flexible Fitting. J. E. Stone, R. McGreevy, B. Isralewitz, and K. Schulten. Faraday Discussions 169:265-283, 2014. 


\section{VMD MDFF Cross Correlation}

Rabbit Hemorrhagic Disease Virus: $702 \mathrm{~K}$ atoms, $6.5 \AA$ resolution

Software, Hardware platform

Chimera, Intel Xeon E5-2687W (2 socket) [1]

VMD, Intel Xeon E5-2687W (2 socket) [1]

VMD-CUDA, Intel Xeon E5-2687W ～+ Quadro K6000 [1,2]

VMD-CUDA, Intel Xeon E5-2698v3 + Tesla P100 [3]

VMD-CUDA, IBM Power8 "Minsky" + Tesla P100 [3]

\section{Runtime, Speedup vs. Chimera, VMD+GPU}

15.860s,
0.779 s,

$20 x$

$0.458 \mathrm{~s}$,

$35 x$

$1.0 \mathrm{x}$

0.090s,

$176 x$

$5.1 x$

$1 \mathrm{x}$

0.080 s,

$198 x$

$5.7 x$

Image credits:

[1] GPU-Accelerated Analysis and Visualization of Large Structures Solved by Molecular Dynamics Flexible Fitting.

J.E. Stone, R. McGreevy, B. Isralewitz, and K. Schulten. Faraday Discussions 169:265-283, 2014.

[2] Early Experiences Porting the NAMD and VMD Molecular Simulation and Analysis Software to GPU-Accelerated OpenPOWER Platforms. J. E. Stone, A.-P. Hynninen, J.C. Phillips, K. Schulten. IIWOPH'16, LNCS 9945, pp. 188-206, 2016.

[3] Results, VMD 1.9.3 November 2016 


\section{Parallel MDFF Cross Correlation Analysis on Cray XK7}

Rabbit Hemorrhagic Disease Virus (RHDV)

\begin{tabular}{|l|l|}
\hline $\begin{array}{l}\text { Traj. frames } \\
\begin{array}{l}\text { Structure } \\
\text { component } \\
\text { selections }\end{array}\end{array}$ & 10,000 \\
\hline $\begin{array}{l}\text { Single-node XK7 } \\
\text { (projected) }\end{array}$ & 336 hours (14 days) \\
\hline 128-node XK7 & $\begin{array}{l}3.2 \text { hours } \\
105 x \text { speedup }\end{array}$ \\
\hline 2048-node XK7 & $\begin{array}{l}19.5 \text { minutes } \\
1035 x \text { speedup }\end{array}$ \\
\hline
\end{tabular}

Calculation of 7M CCs would take 5 years using serial CPU algorithm!



RHDV colored by relative $\mathrm{CC}$
$-0.0032$

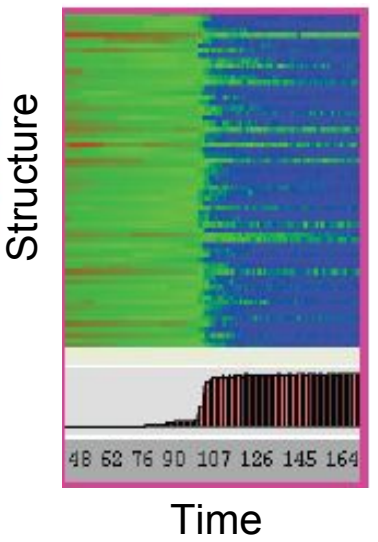

Stone et al., Faraday Discuss., 169:265-283, 2014. 
Adapting VMD for High Fidelity Rendering








Ray Tracing in VMD

- $\quad$ Support for ray tracing of VMD molecular scenes began in 1995

- Tachyon parallel RT engine interfaced with VMD (1999)

- Tachyon embedded as an internal VMD rendering engine (2002)

- $\quad$ Built-in support for large scale parallel rendering (2012)

- Refactoring of VMD to allow fully interactive ray tracing as an alternative to OpenGL (2014 to present, ongoing) 


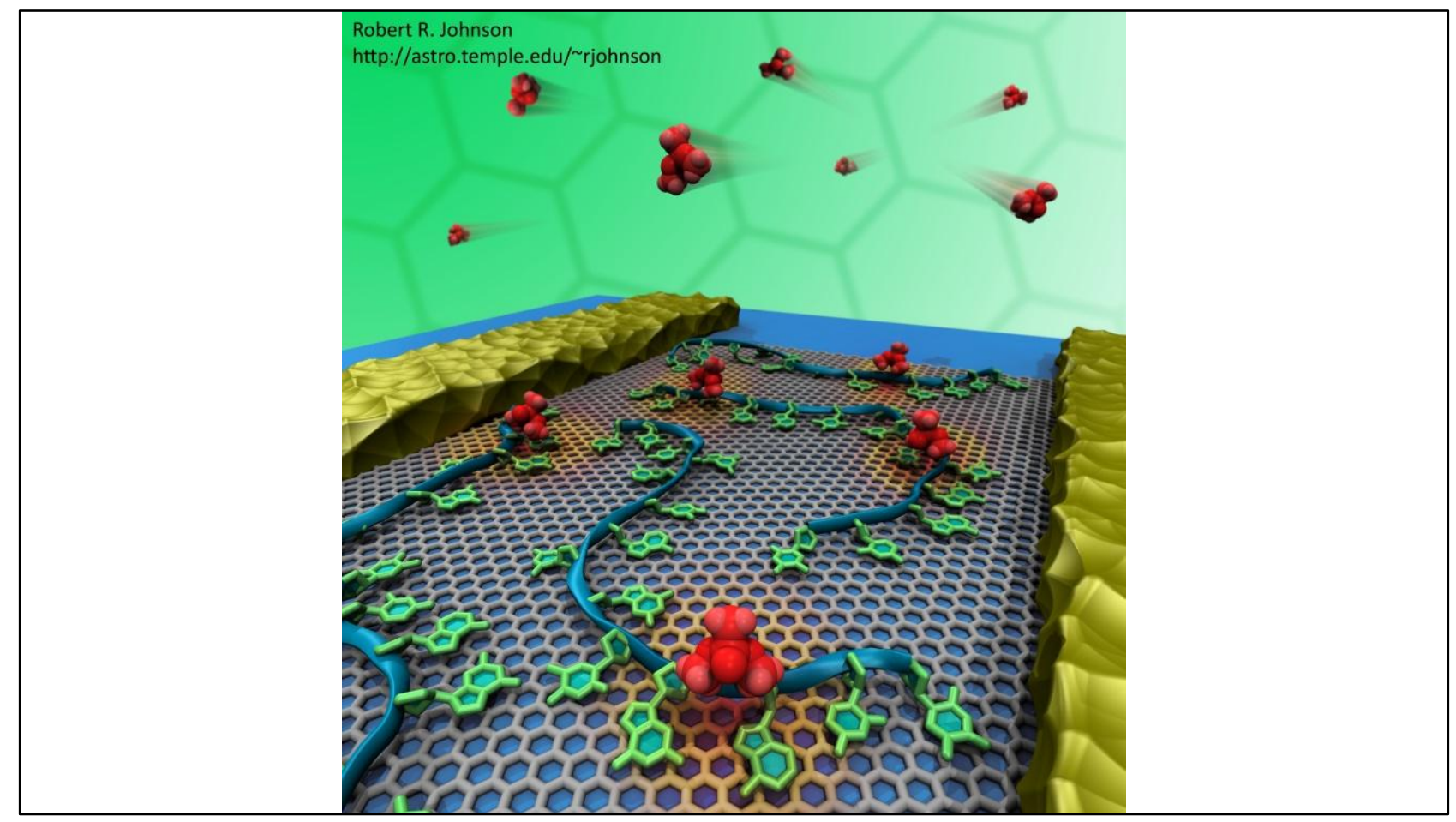

Ray Tracing Performance

- Well suited to massively parallel hardware

- Peak performance requires full exploitation of SIMD/vectorization, multithreading, efficient use of memory bandwidth

- Traditional languages+compilers not yet up to the task:

- Efficacy of compiler autovectorization for Tachyon and other classical RT codes is very low...

- Core ray tracing kernels have to be explicitly designed for the target hardware, SIMD, etc.

Fast Ray Tracing Frameworks

- Applications focus on higher level RT ops

- Parallel SPMD-oriented languages and compilers address the shortcomings of traditional tools

- RT frameworks provide performance-critical algorithms:

- NVIDIA OptiX/CUDA: general RT framework for writing high performance GPU ray tracing engines

- Intel OSPRay/Embree/ISPC: general RT framework and library, includes both basic kernels and full renderer implementations

- AMD FireRays/OpenCL: library of high perf. GPU RT algorithms 


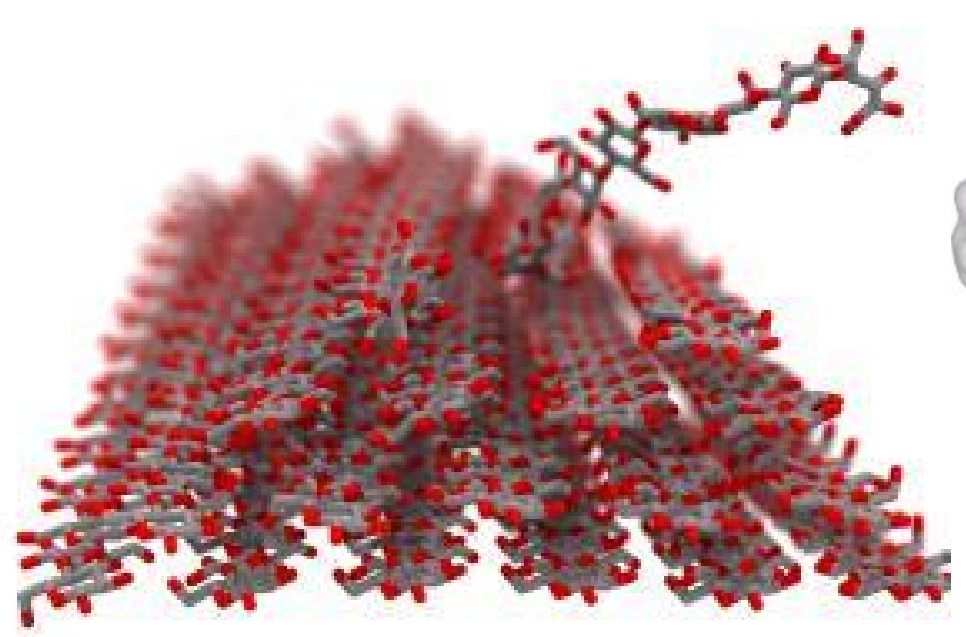

Decrystallization: Interactive Ray Tracing w/ Ambient Occlusion Lighting, Depth of Field Focal Blur

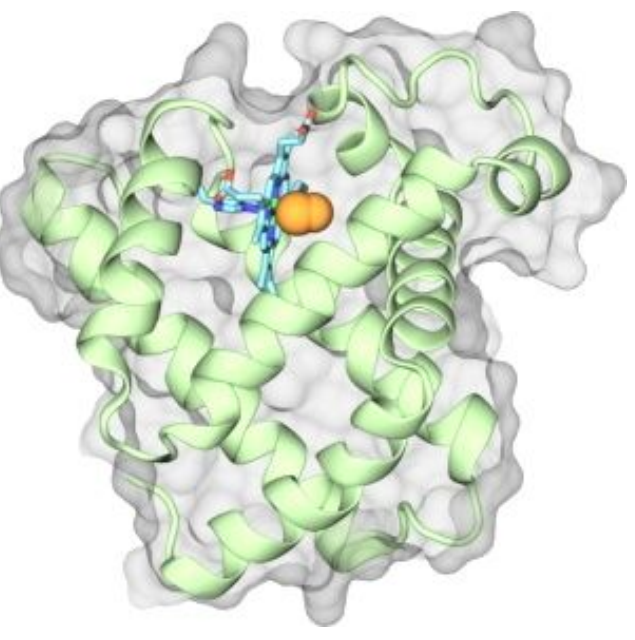

Myoglobin

Diverse Shading and Lighting Approaches 


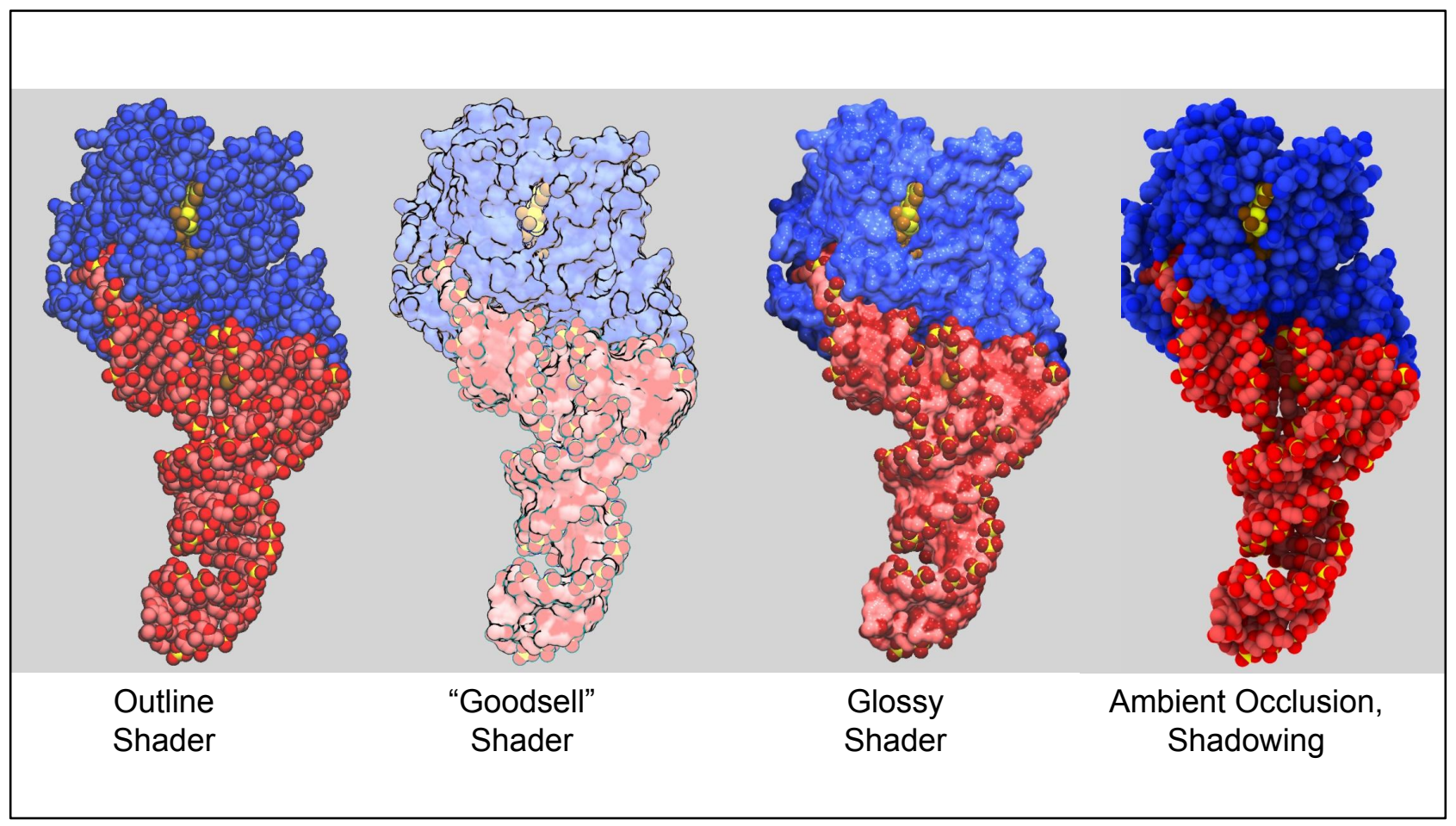

VMD Shading Comparison: EF-Tu 


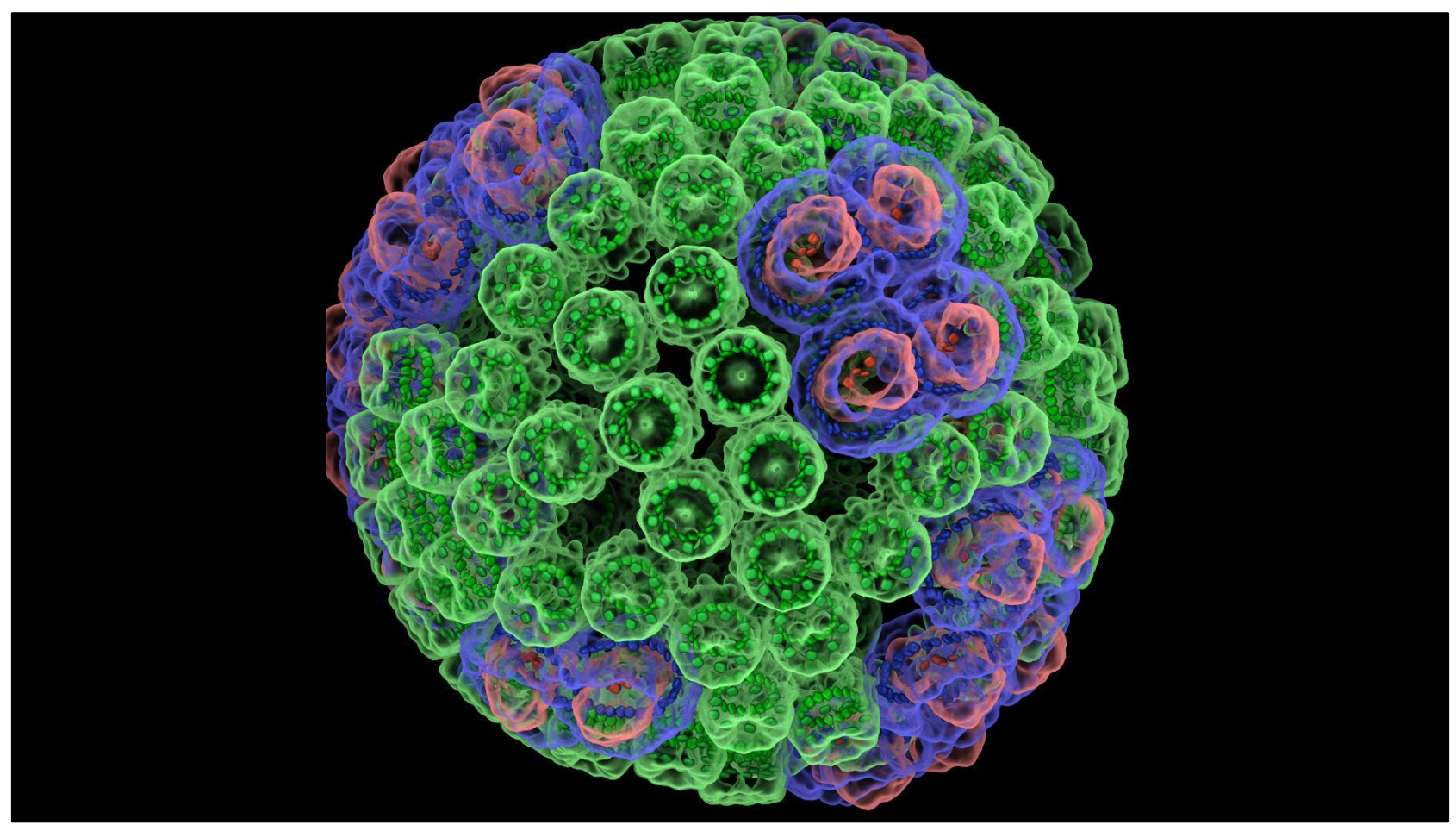

High Fidelity Interactive Visualization

VMD interactive ray tracing

- Interactive ray tracing with progressive refinement of image and lighting quality

- Fully interactive rendering of large structures with advanced lighting features, and WYSIWYG "What you see is what you get" final image output 


\section{VMD-OSPRay Interactive CPU Ray Tracing with Progressive Refinement}
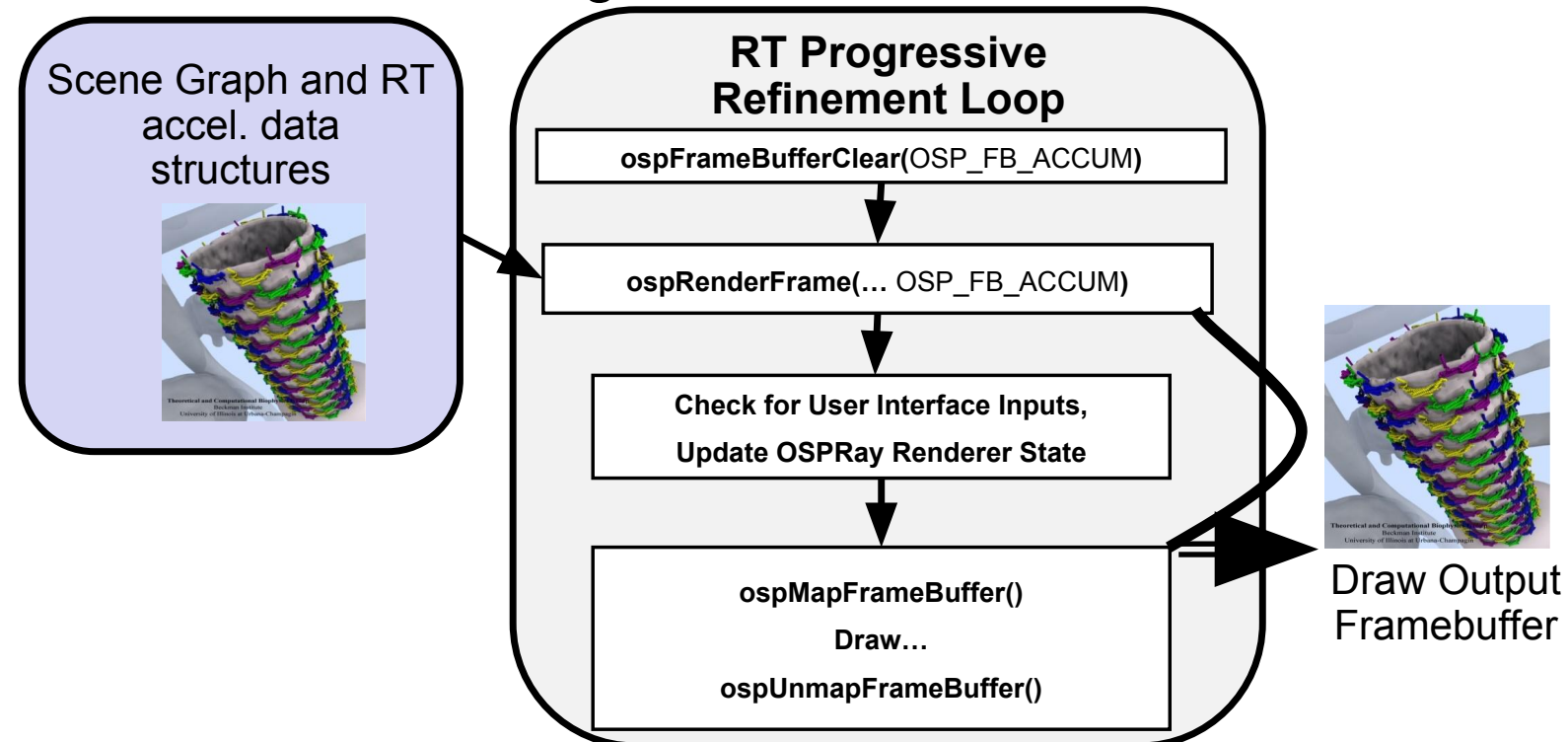


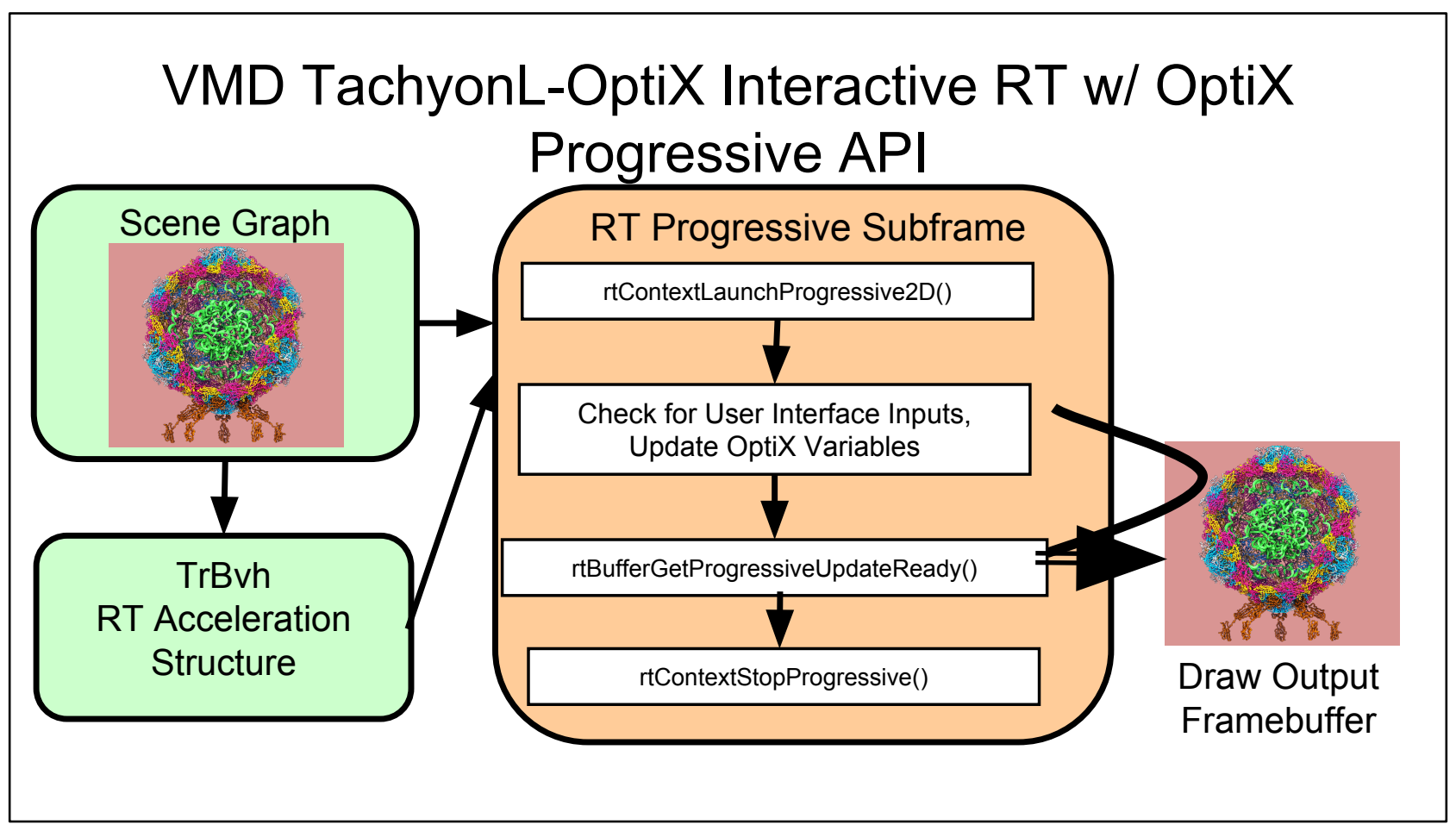









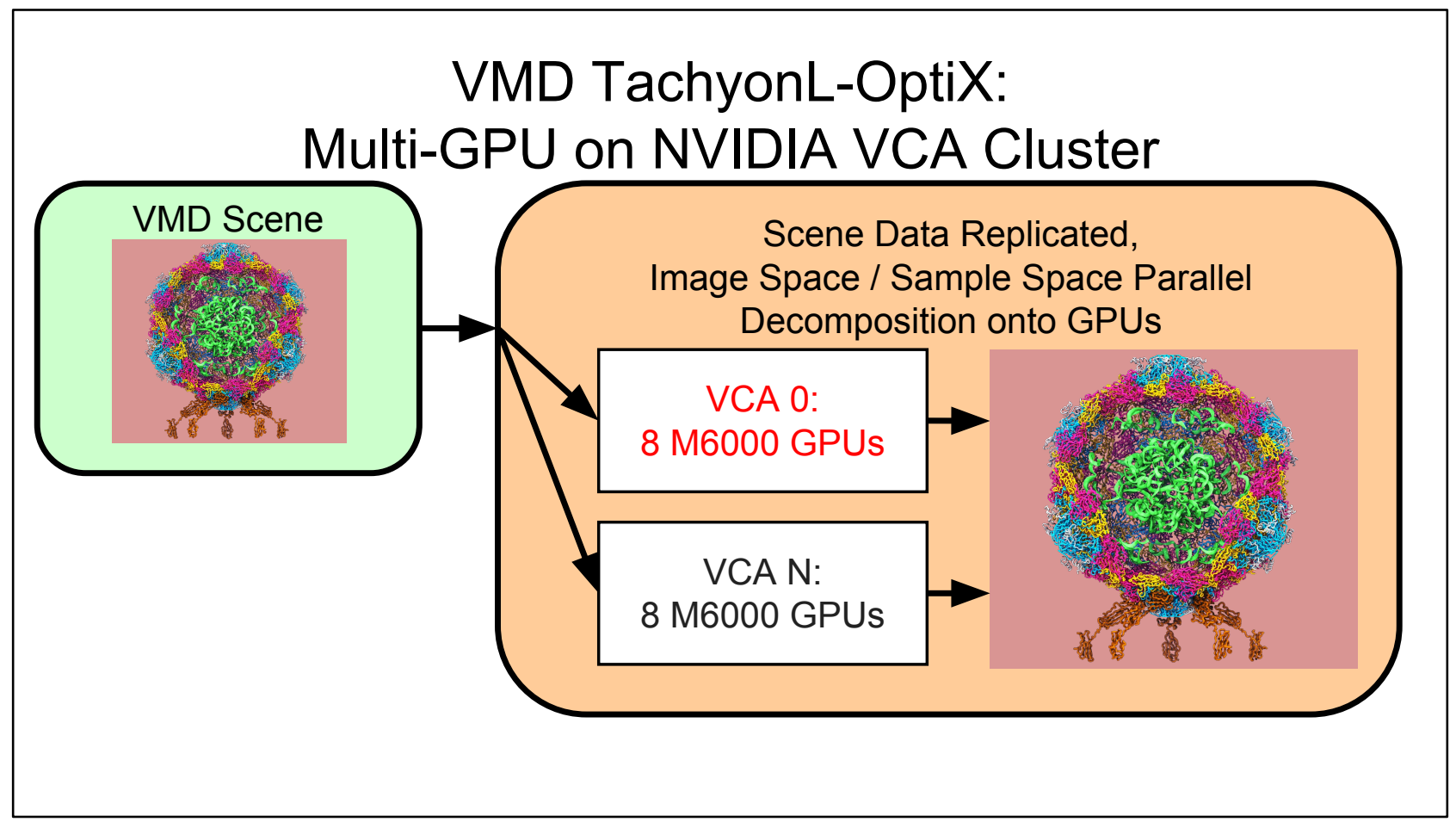




\section{VMD w/ OptiX 4.1}

- Interactive RT on laptops, desktops, and cloud

- Large-scale parallel rendering: in situ or post hoc visualization tasks

- Remote RT on NVIDIA VCA clusters

- Stereoscopic panoramic and full-dome projections

- Omnidirectional VR for YouTube, VR HMDs

- Top-end Pascal Tesla GPUs roughly $2 x$ faster than Kepler

- GPU memory sharing via NVLink on Quadro GP100, Tesla P100

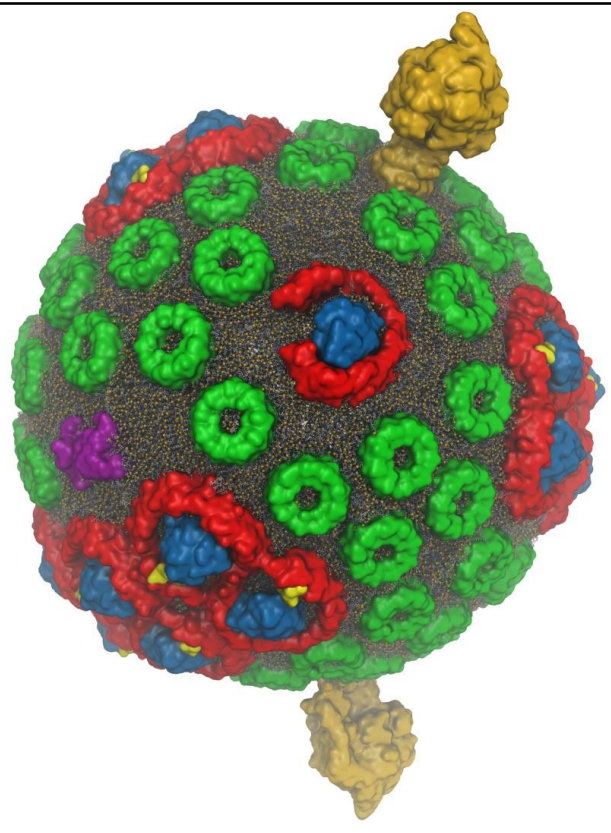

VMD/OptiX GPU Ray Tracing of all-atom Chromatophore w/ lipids.

Credits:

GPU-Accelerated Molecular Visualization on Petascale Supercomputing Platforms. J. E. Stone, K. L. Vandivort, and K. Schulten. UltraVis'13, pp. 6:1-6:8, 2013.

Visualization of Energy Conversion Processes in a Light Harvesting Organelle at Atomic Detail. M. Sener, et al. SC'14 Visualization and Data Analytics Showcase, 2014.

Chemical Visualization of Human Pathogens: the Retroviral Capsids. J. R. Perilla, B.-C. Goh, J. E. Stone, and K. Schulten. SC'15 Visualization and Data Analytics Showcase, 2015.

Atomic Detail Visualization of Photosynthetic Membranes with GPU-Accelerated Ray Tracing. J. E. Stone et al., J. Parallel Computing, 55:17-27, 2016.

Immersive Molecular Visualization with Omnidirectional Stereoscopic Ray Tracing and Remote Rendering J. E. Stone, W. R. Sherman, and K. HPDAV, IPDPSW, pp. 1048-1057, 2016. 
Two lights, no shadows

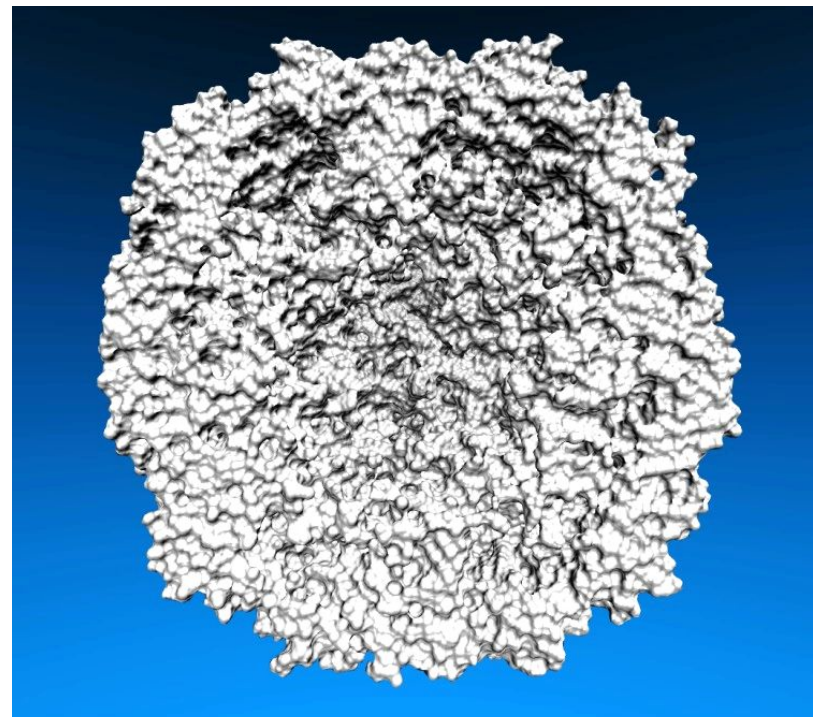

Ambient occlusion lighting \& shadows w/ RT

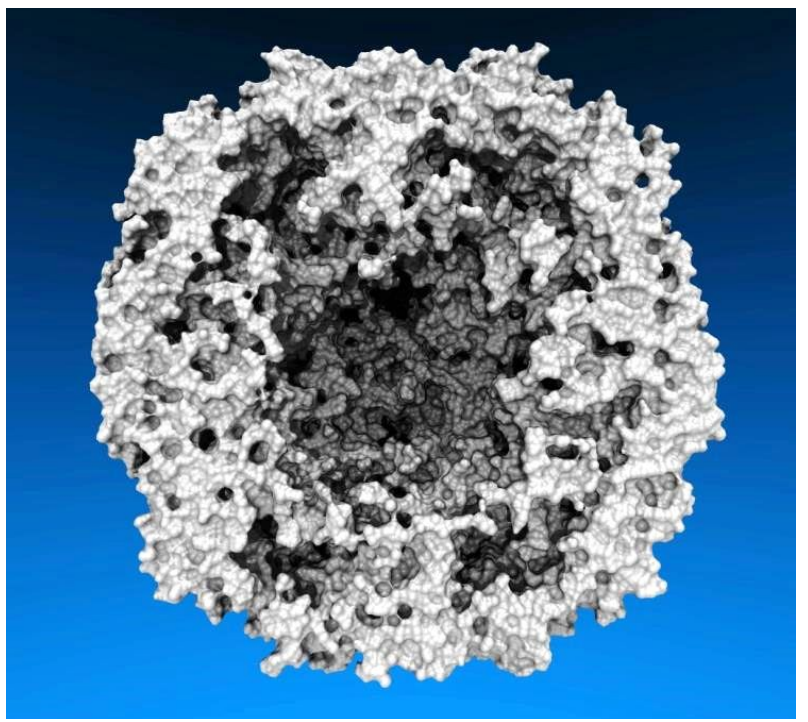

Interactive Ray Tracing, Lighting Comparison: STMV Capsid 


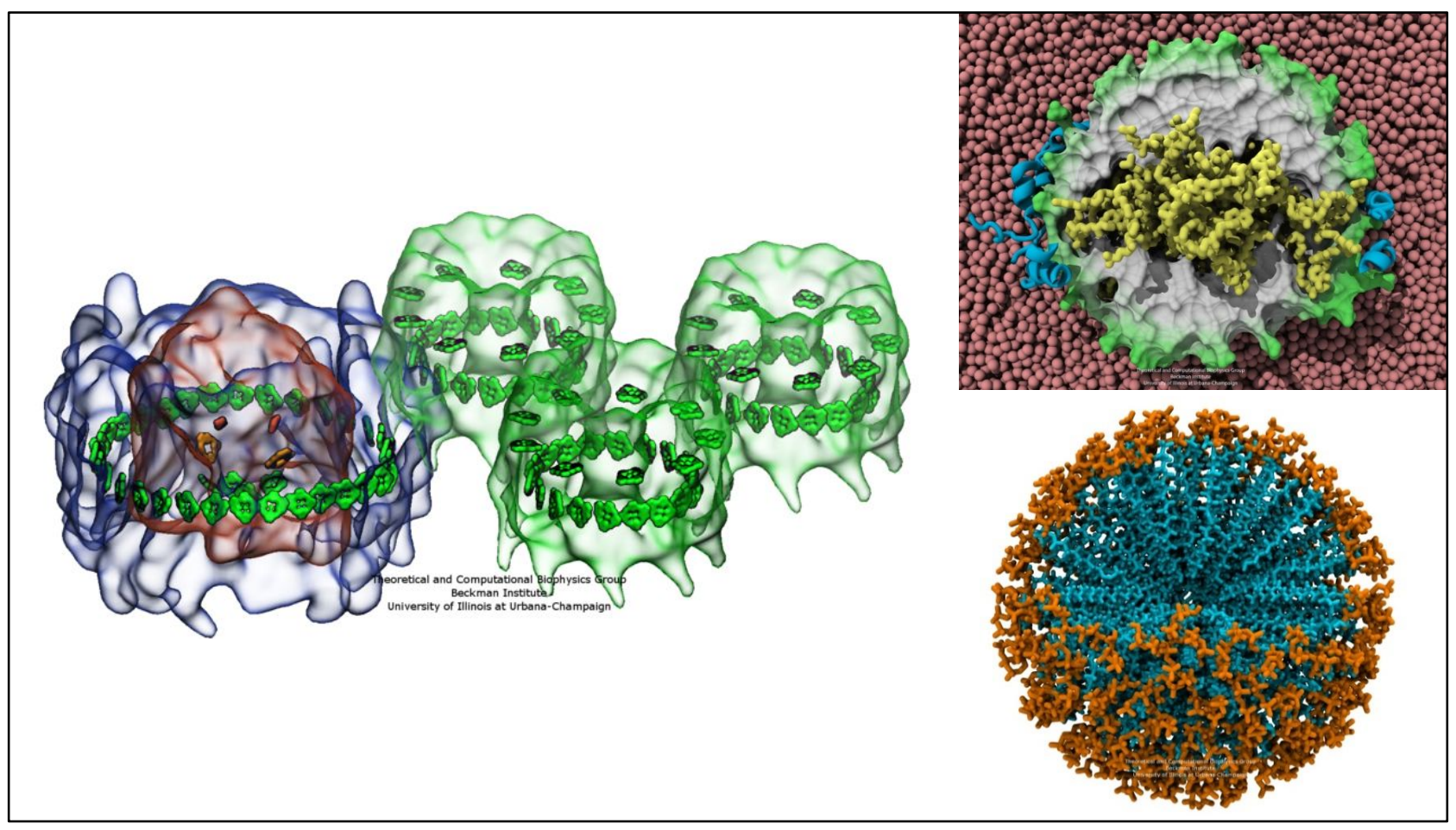

Benefits of Advanced Lighting and Shading Techniques

- Exploit visual intuition

- Spend computer time in exchange for scientists' time, make images that are more easily interpreted 


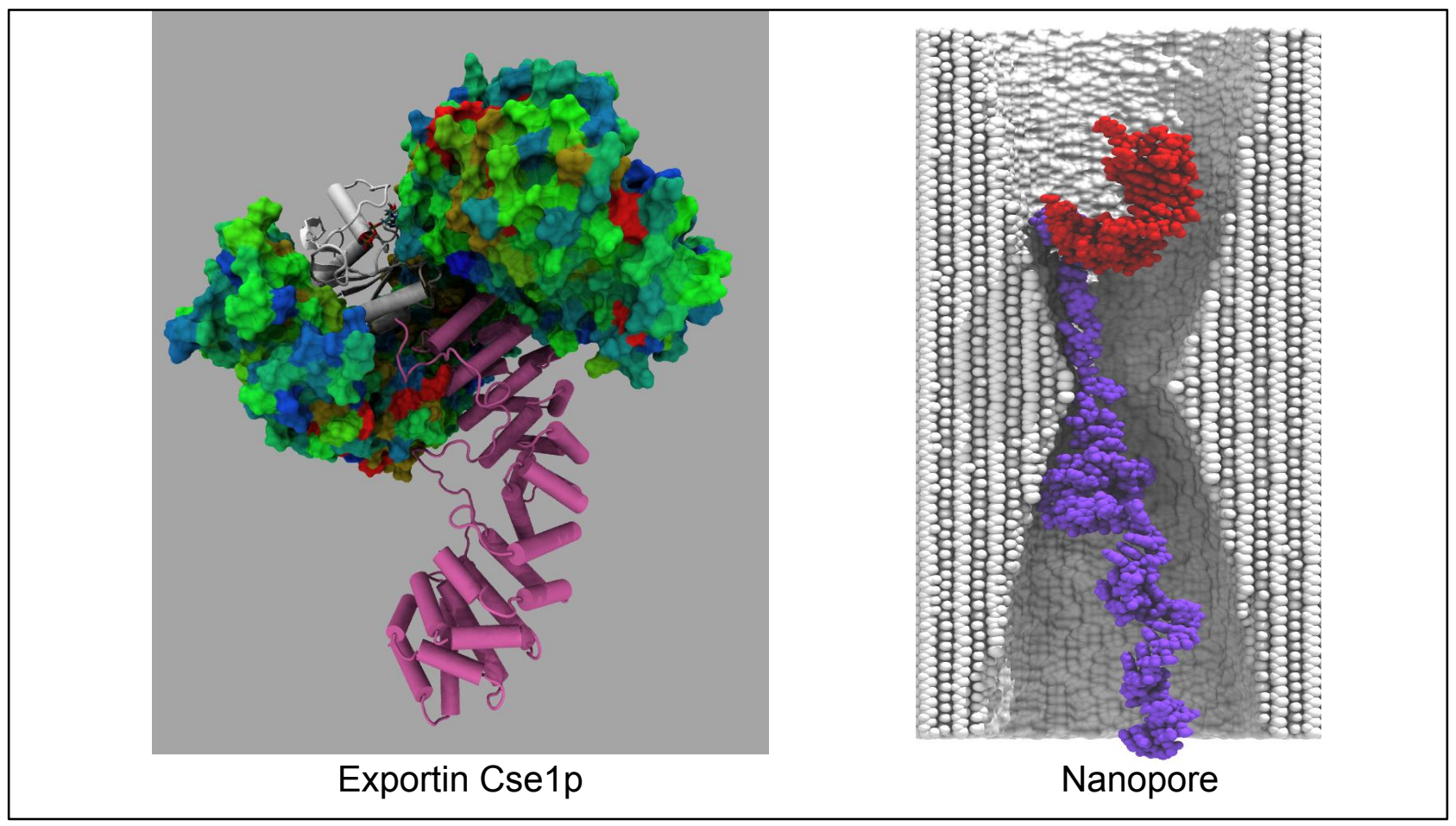

Benefits of Advanced Lighting and Shading Techniques

- Exploit visual intuition

- Spend computer time in exchange for scientists' time, make images that are more easily interpreted 









Geometrically Complex Scenes

Ray tracing techniques well matched to molecular visualization needs:

- $\quad$ Curved geometry, e.g. spheres, cylinders, toroidal patches, easily supported

- $\quad$ Greatly reduced memory footprint vs. polygonalization

- Runtime scales only moderately with increasing geometric complexity

- Occlusion culling is "free", RT acceleration algorithms do this and much more 


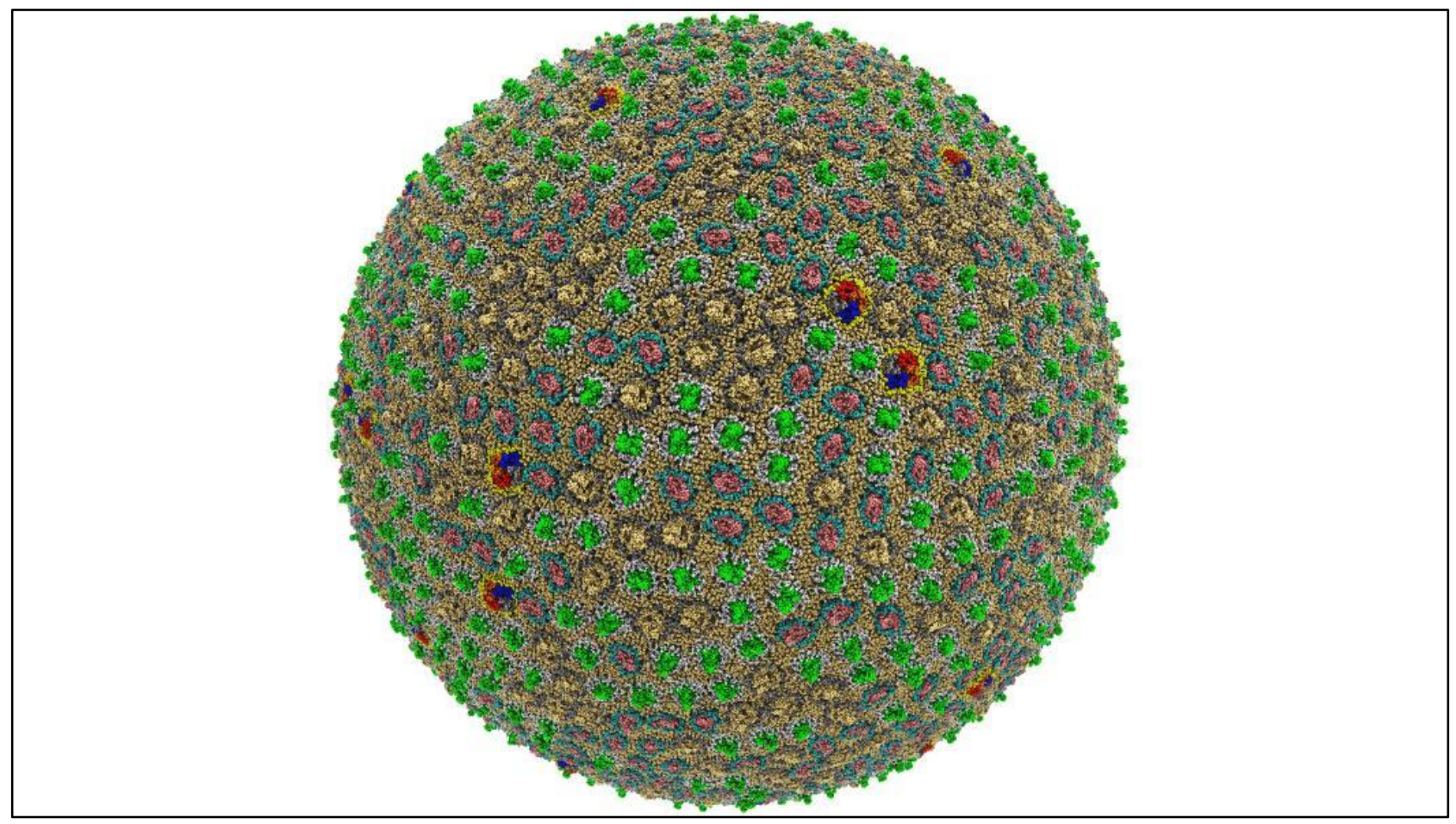

Preparation, Visualization, Analysis of All-Atom Cell-Scale Simulations

- $200 \mathrm{~nm}$ spherical envelope

- Membrane with $\sim 50 \%$ occupancy by proteins

- $63 \mathrm{M}$ atoms in envelope model

- Interactive ray tracing on CPUs and GPUs

- Parallel analysis, visualization w/ MPI

Credits:

Atomic Detail Visualization of Photosynthetic Membranes with GPU-Accelerated Ray Tracing. J.E. Stone et al., J. Parallel Computing, 55:17-27, 2016.

High Performance Molecular Visualization: In-Situ and Parallel Rendering with EGL. J.E. Stone et al. IEEE High Performance Data Analysis and Visualization, IPDPSW, pp. 1014-1023, 2016. 


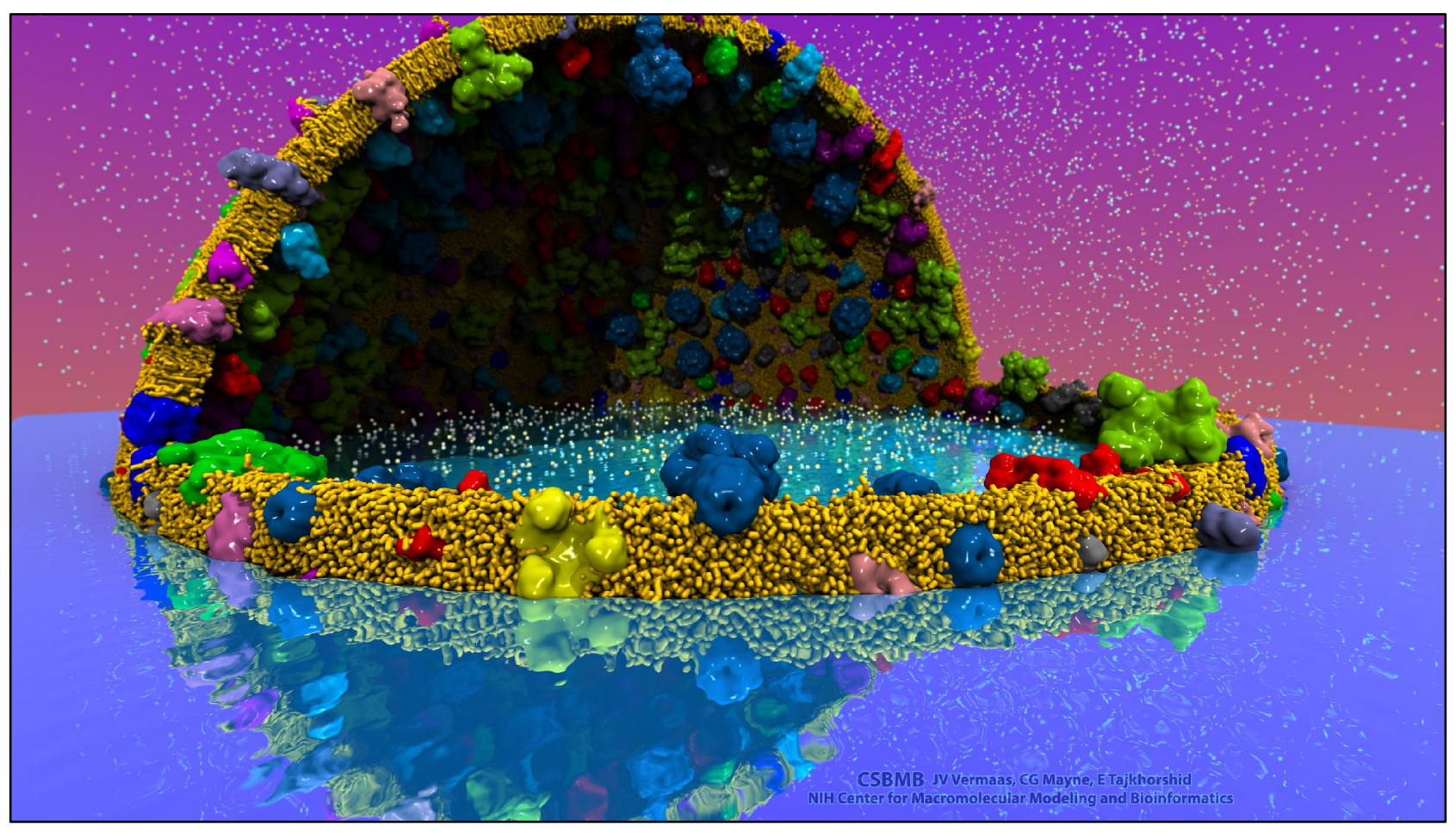

Proto-Cell Rendered with VMD+OptiX

- $113 \mathrm{M}$ particles

- $\quad 1,397$ copies of 14 different membrane proteins

- $\quad$ Preparing for simulations on pre-exascale computers 




Stereoscopic Panorama Ray Tracing

- Commoditization of HMDs excellent opportunity to overcome cost/availability

- This leaves many challenges still to solve:

- Uls, multi-user collaboration/interaction

- Accommodate limitations and idiosyncracies of commercial HMDs

- Support for remote visualization

- $\quad$ Rendering perf for large molecular systems

- $\quad$ Render $360^{\circ}$ images and movies for VR headsets such as Oculus Rift, Google Cardboard, YouTube VR

- Ray trace omnidirectional stereo spheremaps or cubemaps for very high-frame-rate reprojection and display via OpenGL texturing

- Stereo requires spherical camera projections poorly suited to rasterization

- Benefits from OptiX multi-GPU rendering and load balancing, remote visualization 


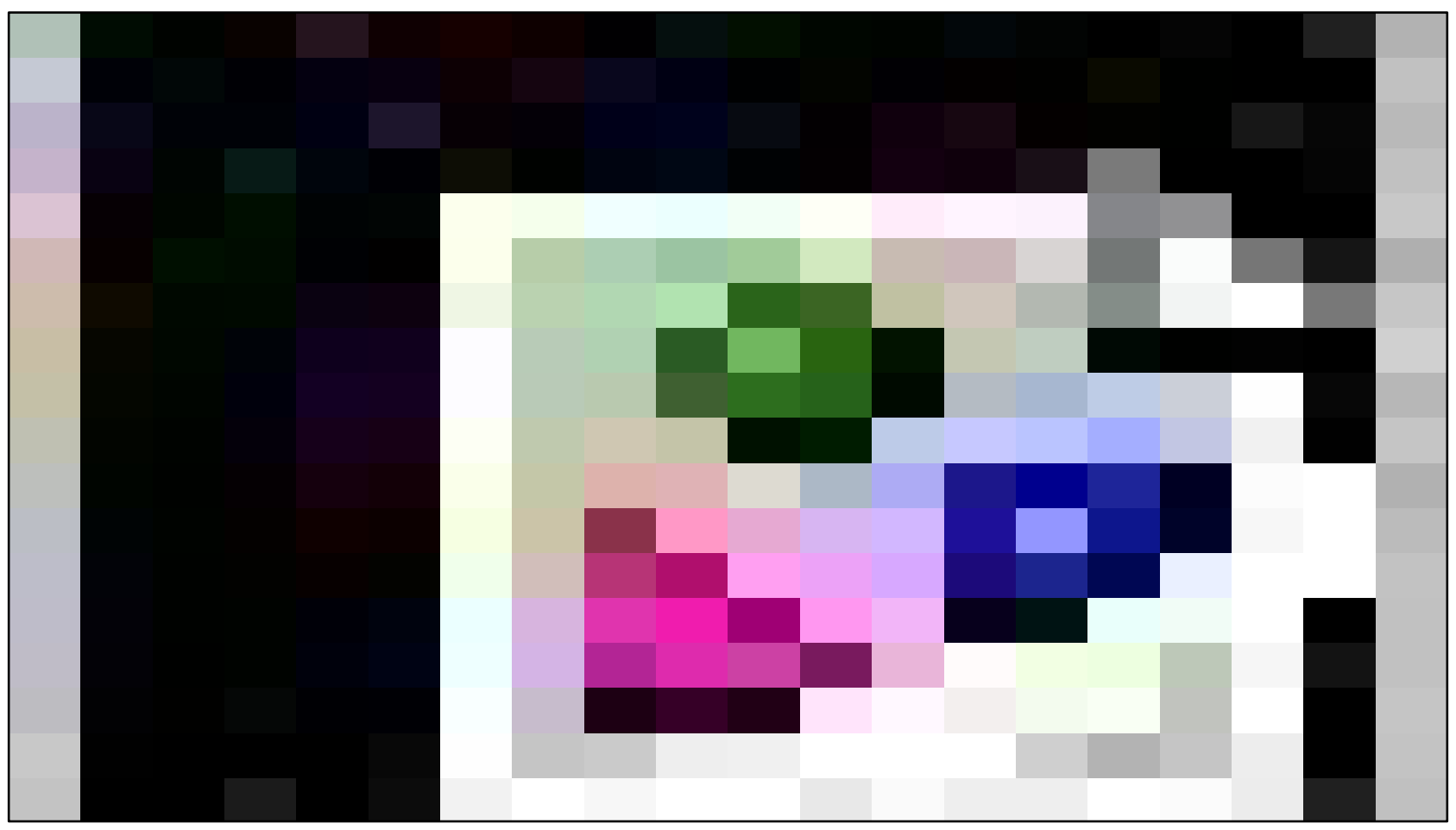

Credits:

Immersive Molecular Visualization with Omnidirectional Stereoscopic Ray Tracing and Remote Rendering. J. E. Stone, W. R. Sherman, and K. Schulten. High Performance Data Analysis and Visualization Workshop, IEEE International Parallel and Distributed Processing Symposium Workshops (IPDPSW), pp. 1048-1057, 2016. 


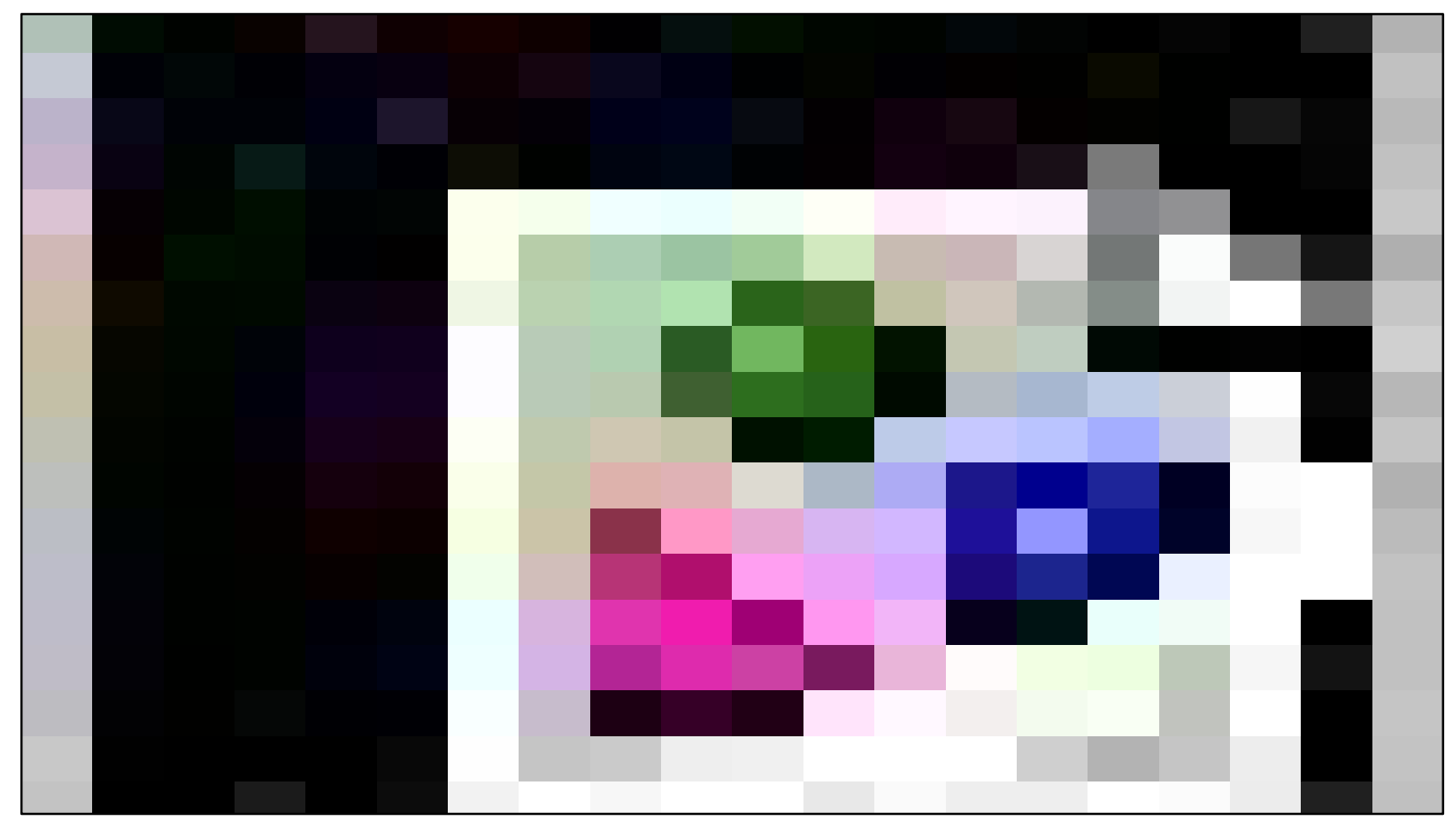




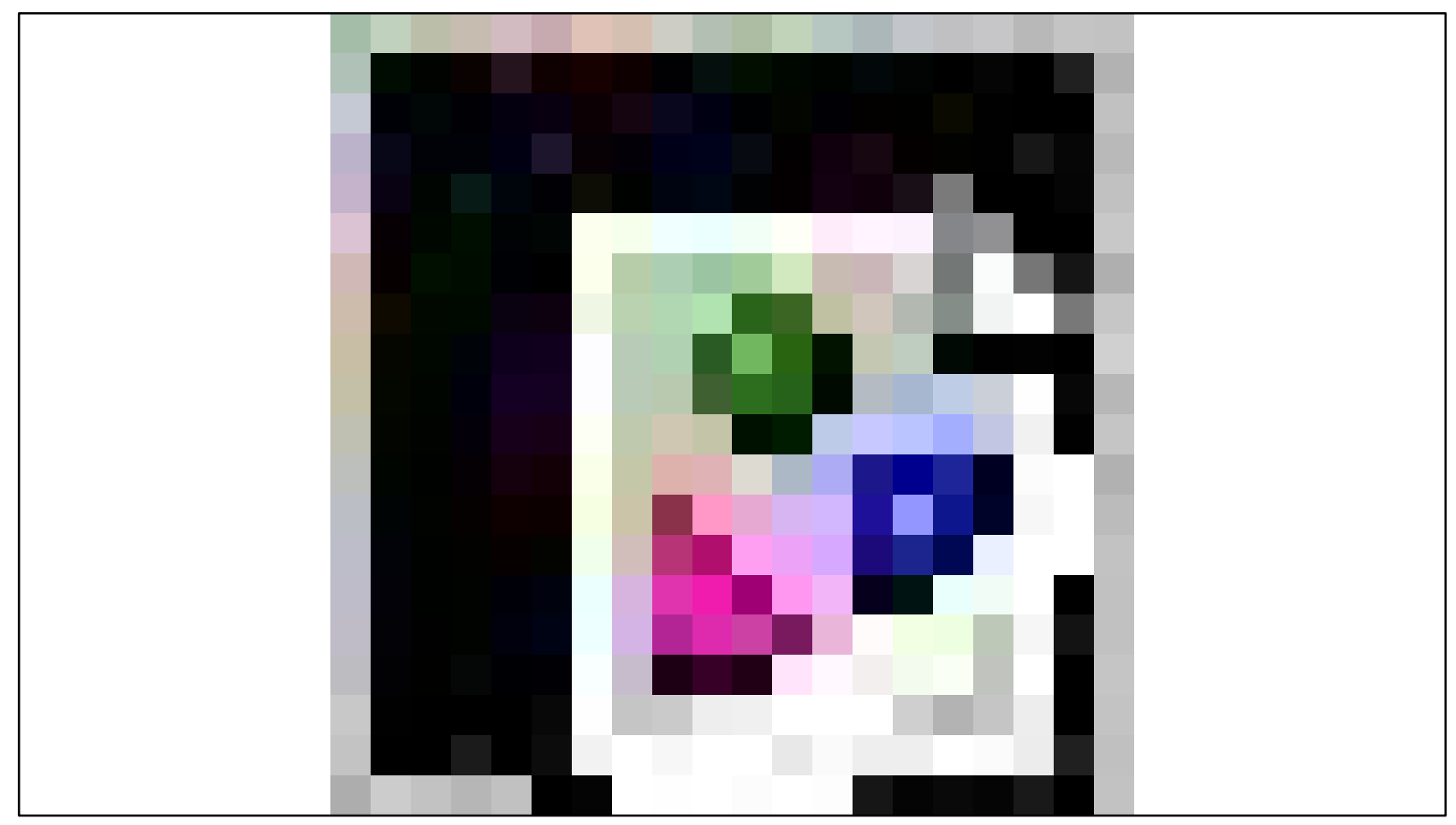

\section{VMD Planetarium Dome Master Camera}

- $\quad$ RT-based dome projection -- rasterization poorly suited to non-planar projections

- Fully interactive RT with ambient occlusion, shadows, depth of field, reflections, and so on

- $\quad$ Both mono and stereoscopic

- No further post-processing required 


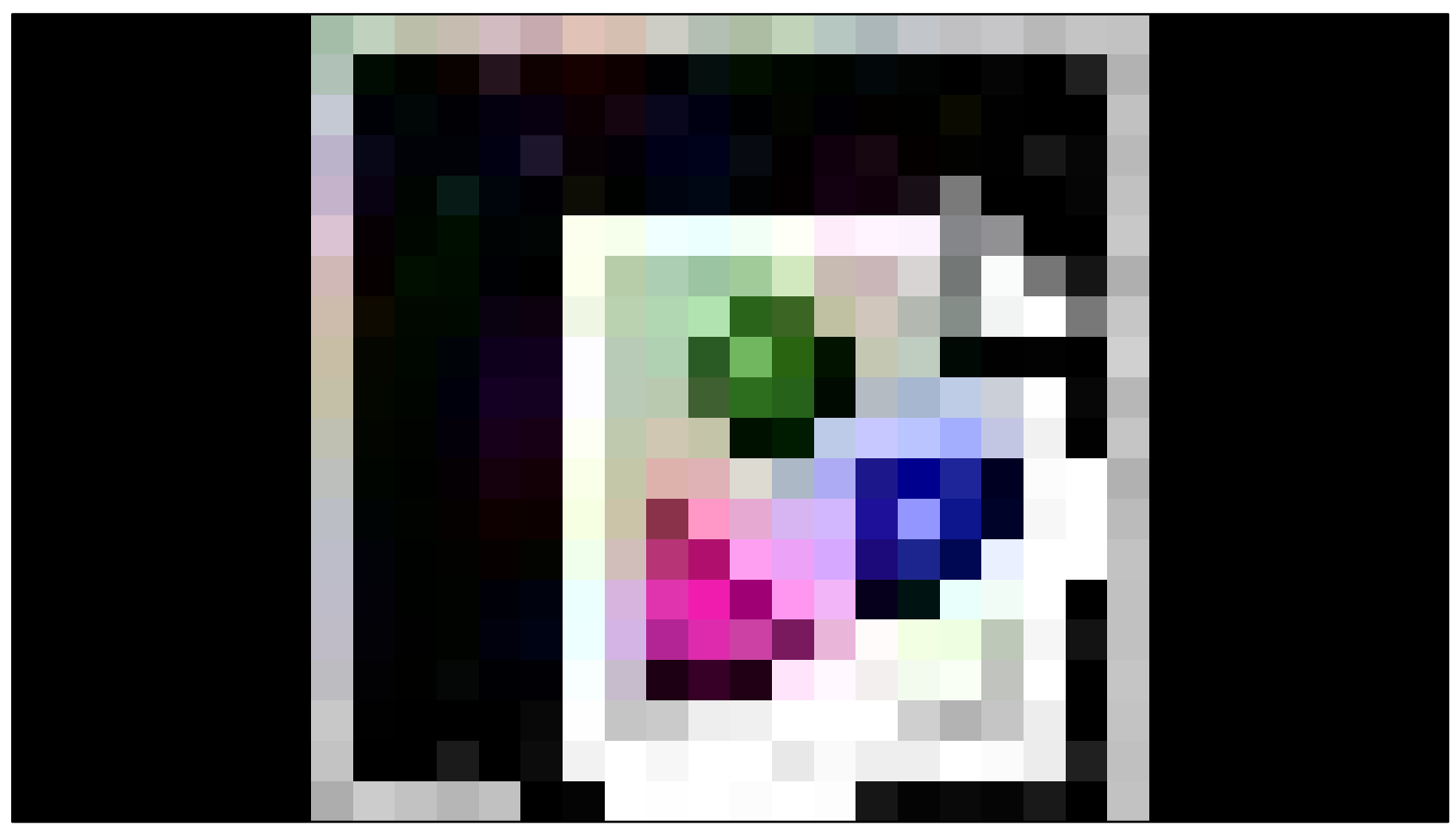

VMD Strengths for Cinematic Visualization

- $\quad$ Built-in high performance ray tracing engines in VMD provide an excellent starting point...

- Maintain $100 \%$ of the domain-specific VMD molecular visualization capabilities through the entire process to final frame rendering, no need to export or re-export molecular scene to intermediate geometry/scene files that lose the concept of "what" the geometry represents...

- Makes it easy for science-driven decisions to be made later/deeper in the visualization process than they would be otherwise

- $\quad$ Ability to run in parallel on clusters and supercomputers makes it easy to handle large scale visualization and final frame rendering workloads

- $\quad$ Built-in scripting (Tcl, Python), and runtime-loaded plugins (compiled shared libraries) make it possible to extend/customize VMD effectively 


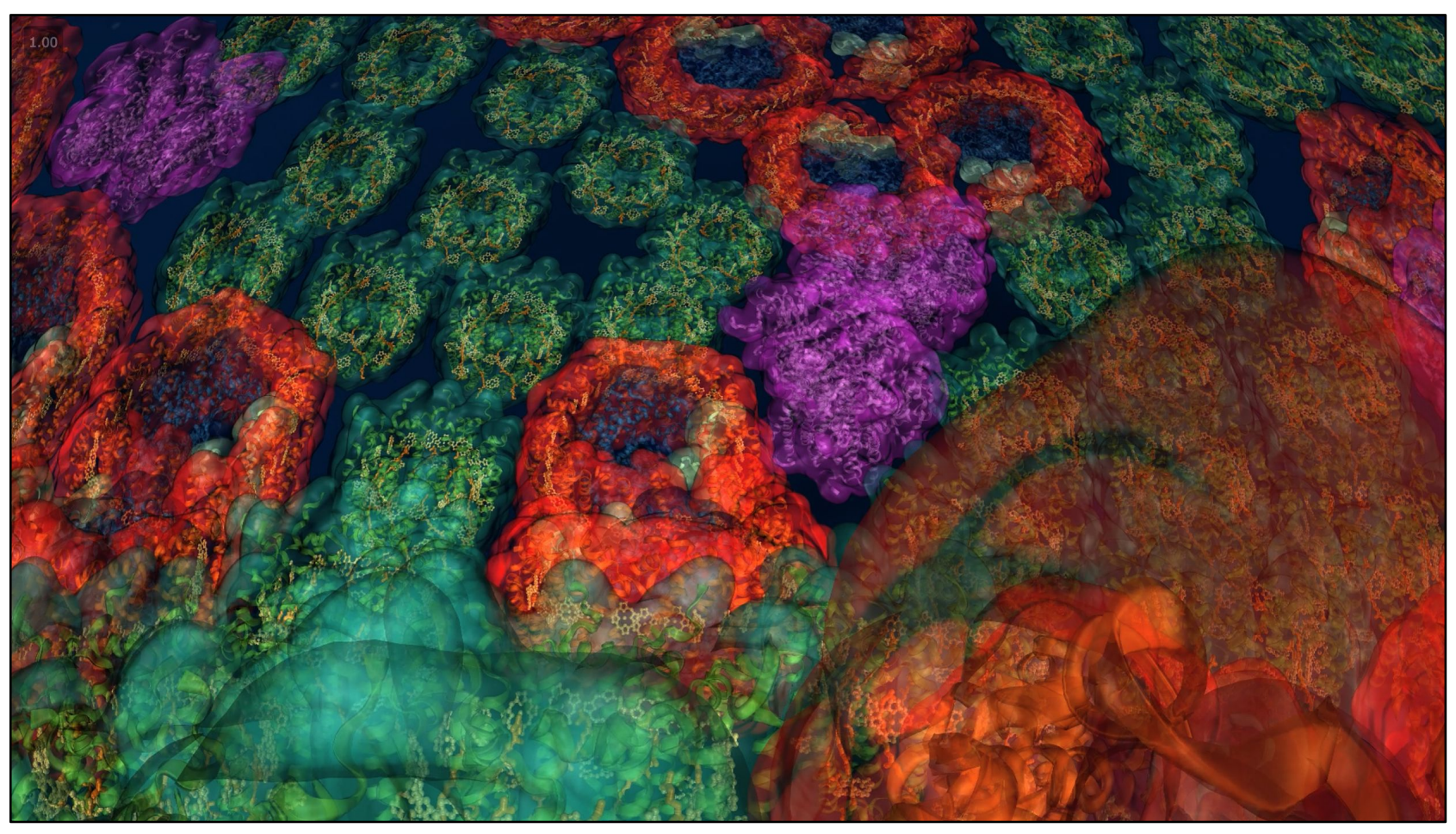

VMD Limitations for Cinematic Visualization

- $\quad$ Built-in high performance ray tracing engines in VMD provide an excellent starting point...

- $\quad$.... but, everything about VMD and its design was intended to optimize routine scientific visualization work, and many of these choices deliberately avoided the introduction of complexity in the user interface

- $\quad$ The purposeful simplicity in parts of the VMD interface makes it hard to use it as-is to produce what we would think of as "cinematic" camera paths

- $\quad$ The kinds of shading, texturing, and surface properties implemented in VMD itself are easy to use for routine scientific tasks, but they lack the full artistic controls that a VFX production rendering system would offer 


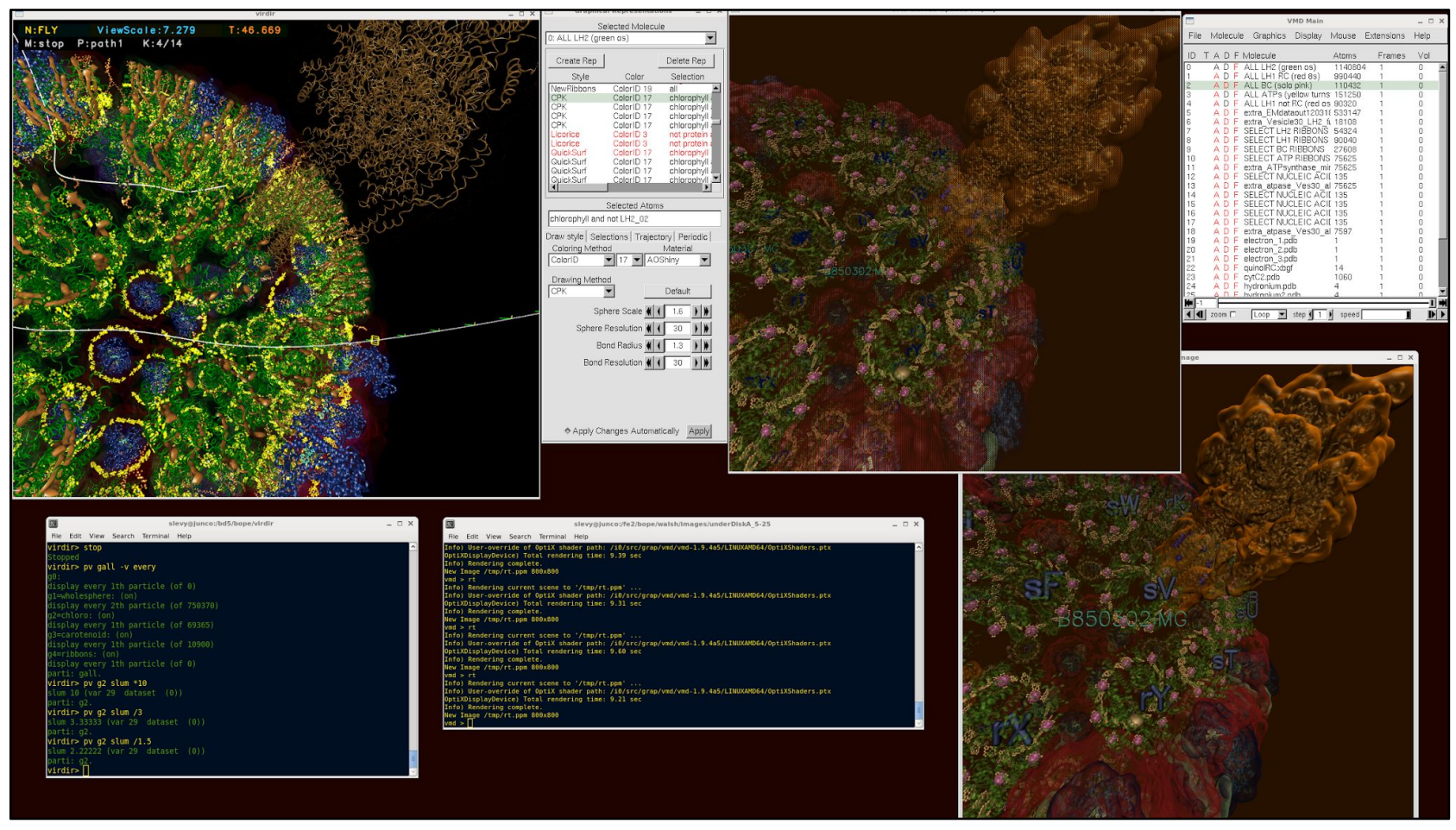

By linking VMD with Virtual Director with some custom scripting, we can address many of the (purposeful) limitations in the VMD user interfaces and the camera controls that it exposes by driving it instead through virtual director. Virtual Director provides the kinds of pre-visualization and camera path planning tools that VMD doesn't specialize in. Together the two tools are combined to yield a cinematic visualization workflow that maintains key strengths and efficiencies associated with performing rendering within VMD, but with the familiar cinematic visualization interfaces provided by Virtual Director. 




Virtual director preview of camera path past representations of chromatophore data generated by VMD. 
Techniques tor Visualization

These techniques have been used by the AVL to efficiently create the most meaningful visuals. 


\section{Techniques: Abstraction and Representation}

While rendering visualizations with photorealistic lighting and materials can add to the drama and immersiveness of an experience, it's often a bad idea to render computational data as realistically as possible. 


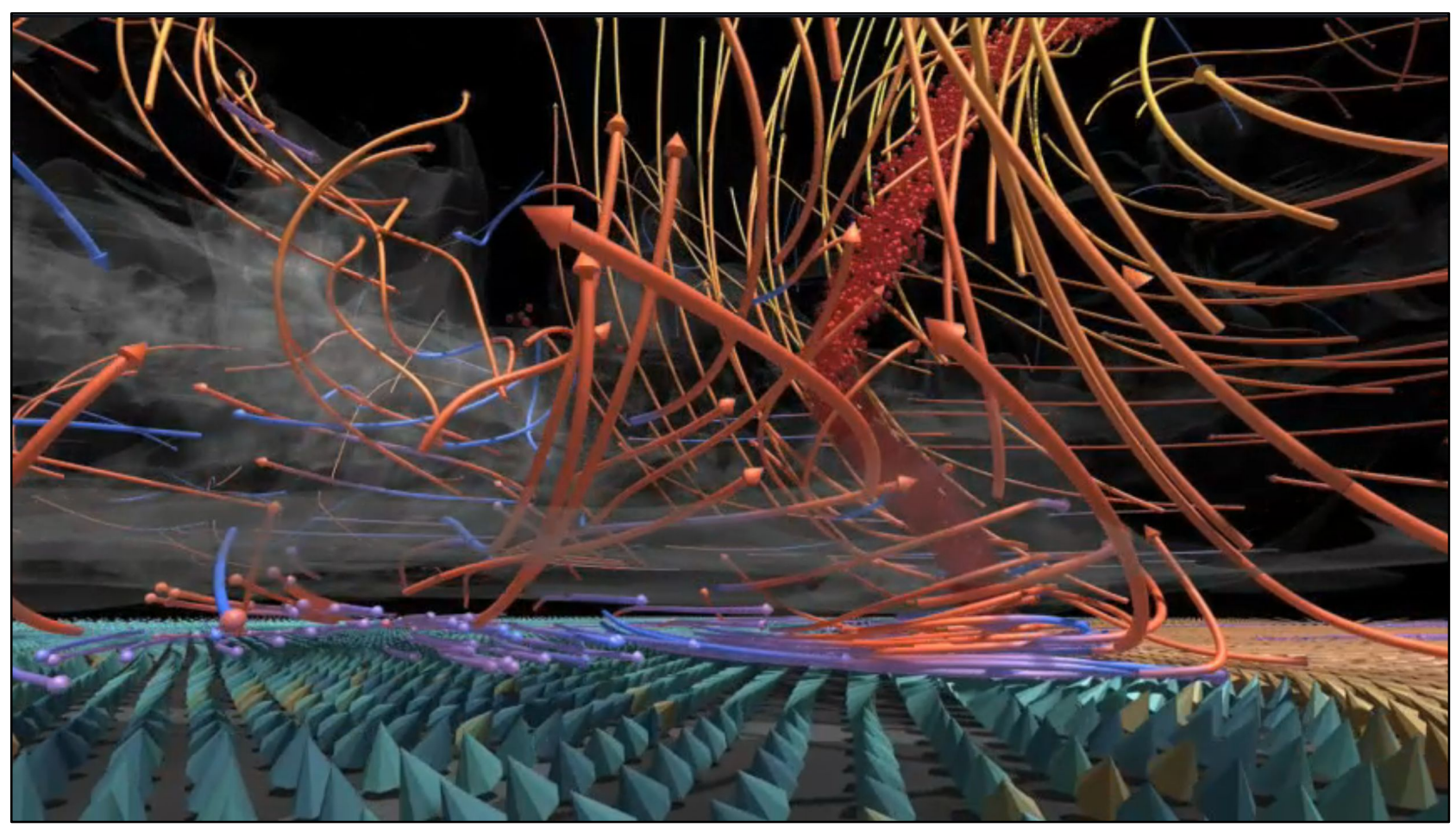

For example, if we had rendered the rain and clouds from this simulation as true-to-life as possible, the screen would be an incomprehensible blur of gray fog. In fact, this simulation was started from the same conditions as a real tornado-forming storm in which stormchasers were collecting data, but it wasn't until we started visualizing the data that they even discovered there might have been two cyclones hidden in the clouds. 


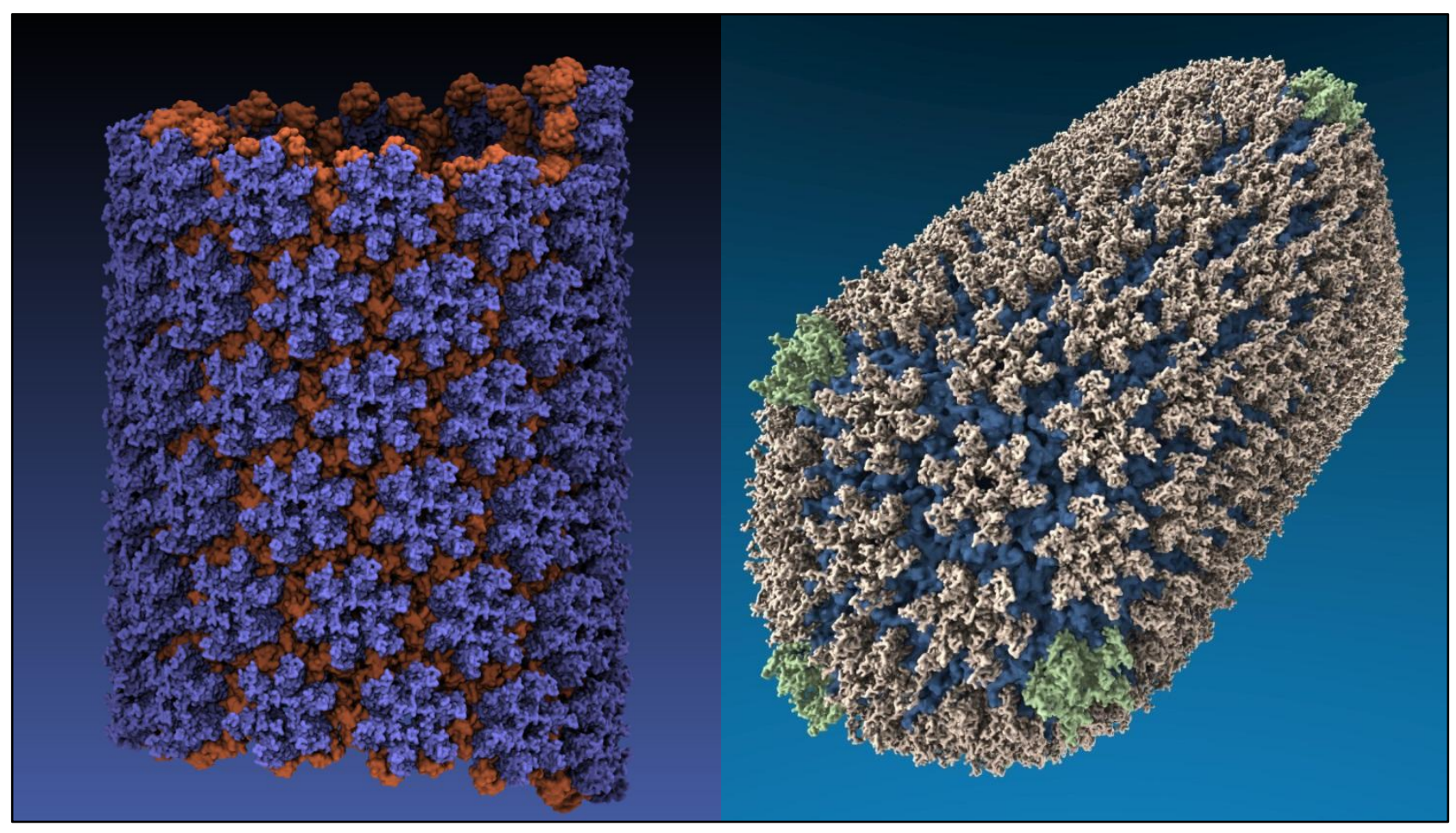

Similarly, all-atom molecular simulations are digital boxes jam-packed with atoms, most of which are filler solution. VMD takes these boxes of incomprehensibly arranged atoms and represents them as bumpy surfaces that are associated with each other by molecular characteristics.

These images show an all-atom HIV capsid simulation with up to 64 million atoms run on the Blue Waters supercomputer. 


\section{$\mathrm{F}=\mathrm{ma}$}

Communicating science at all levels of education depends on abstraction. Abstractions come in many shapes and levels of simplicity. Some abstractions are strong because they rely on an established language that is accumulated in students' minds through years of formal education. For instance, the equivalence of Newton's Second Law of Motion can be abstracted to symbols which in turn represent the English words "force", "mass", and "acceleration" by sharing the words' first letter. 


\section{Abstraction}

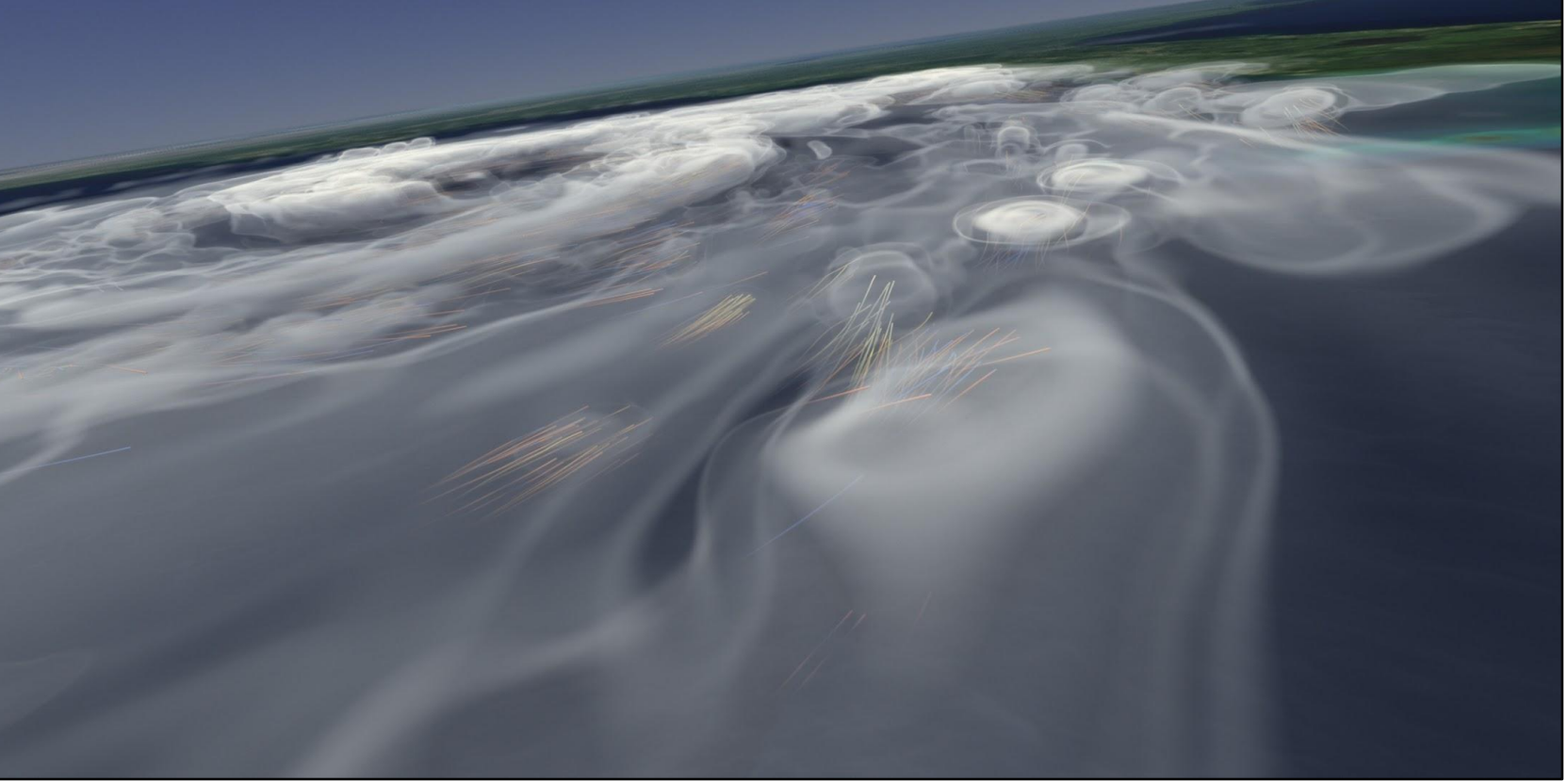

Other abstractions are strong because our intuition can draw analogies to more familiar objects. In this visualization of a simulation of Hurricane Katrina, we use an abstraction called "isovolumes" to see the space inside the rain clouds circling the eye of the storm. Isovolumes tend to look similar to $\mathrm{x}$-ray images or cross-sectional diagrams, which give viewers a familiar footing from which to interpret the images. The streamtubes inside the clouds which represent the wind direction are also reminiscent of windsocks. 


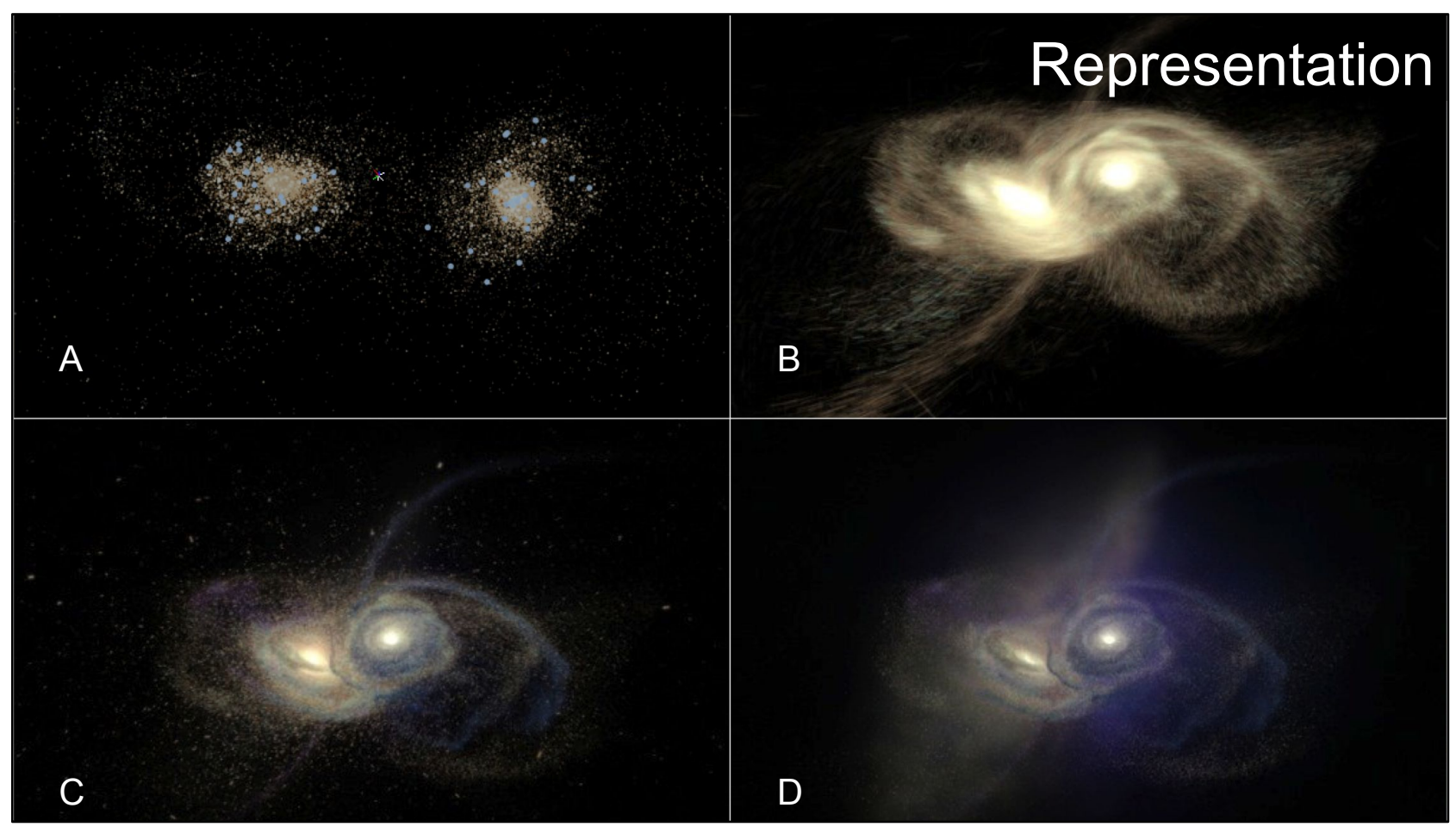

The means by which we represent computational data is largely subjective - visualization designers must decide what visual metaphors are most evocative of the familiar. This can vary wildly depending on the audience - what is familiar can change based on audiences of different ages, cultures, and other demographics.

This image shows 4 different renderings of the exact same galaxy collision simulation represented as:

(A) An interactive pre-visualization of just the stars, showing recently-born stars in bright blue to identify where rapid star formation is happening.

(B) Just the stars, with exaggerated motion blur so that even a still picture suggests how things are moving.

(C) Stars and gas together, with different color choices associated with the original galaxy each parcel of gas came from.

(D) Stars and gas together emphasizing where the gas is going, showing that the gas moves very differently from the stars as the collision proceeds.

Each representation explains different, complementary aspects of the simulated system. Our goal for cinematic sequences featuring these datasets is to clearly describe many of these aspects, with particular emphasis on the aspects relevant to the documentary narrative. 


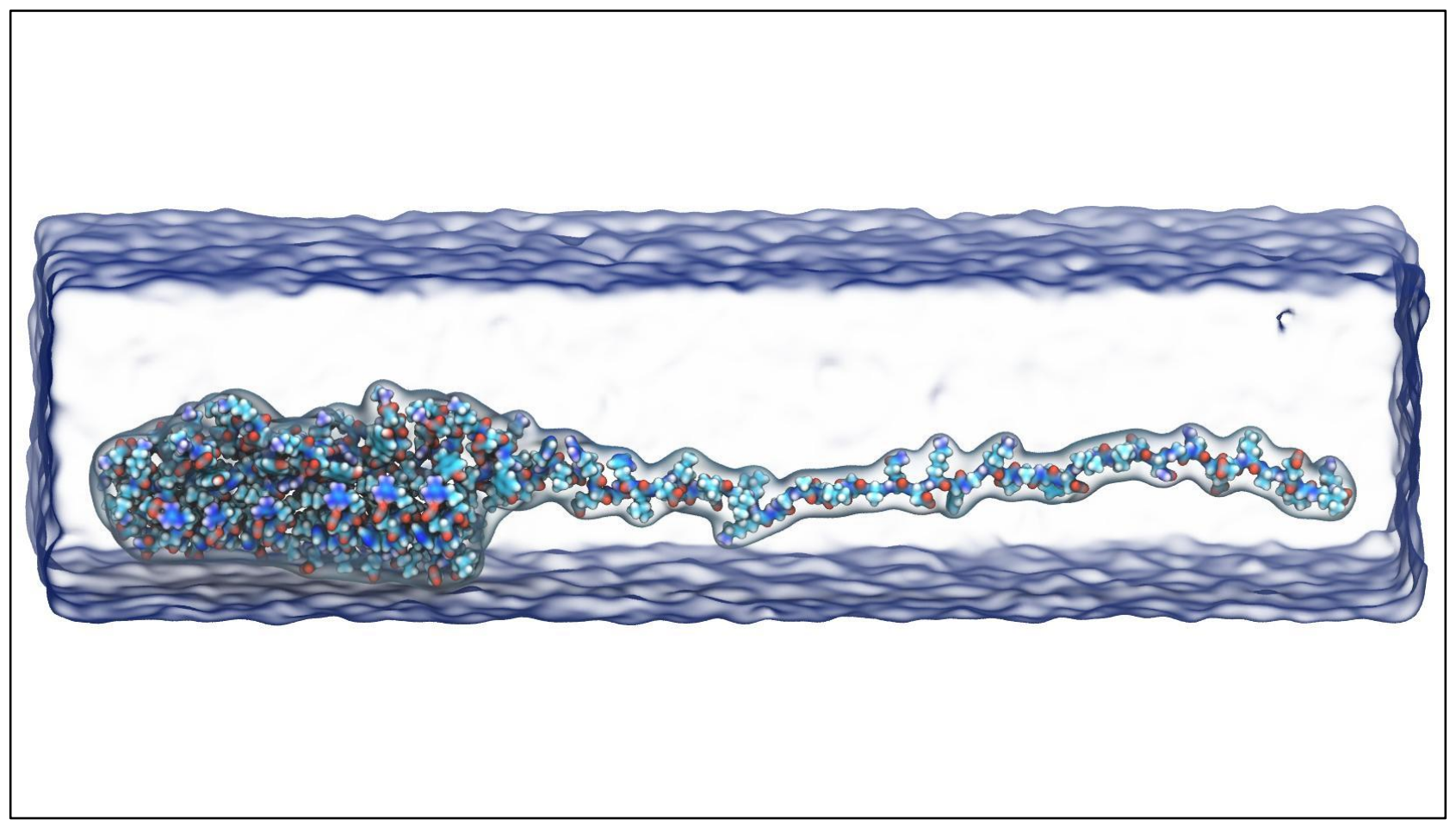

Similarly, molecular representations provide different levels of abstraction from atomic detail to higher level organizational structure. Molecules can be represented as:

- Direct display of atomic structure

- Schematic representations

- Map data to color, texture

- Display data topologically, as graphs, plots

- Combine multiple graphical representations and display modalities together simultaneously

VMD provides these methods for creating molecular representations:

- $\quad$ Atoms, VdW spheres, bonds, ball-stick, ...

- Molecular orbitals (quantum chemistry)

- Molecular surfaces

- Coarse-grained "beads"

- Ribbons, secondary structure, "cartoon" reps, RNA/DNA 


\section{Techniques: Isolation and Simplification}

Abstraction is an important technique for visualization design, but is not enough on its own. Computational models are often extremely geometrically dense and have many overlapping attributes. Carefully choosing what not to show is vitally important. 




Creating streamtubes to show the flow of a vector field can become almost infinitely dense. But if we render so many streamtubes that they occlude the environment or other geometry, we lose context. Sometimes vector fields will attract streamtubes into one region and prevent the tubes from ever reaching other interesting regions. Isolating regions of interest with streamtubes is typically a very iterative process that requires lots of evaluation. 


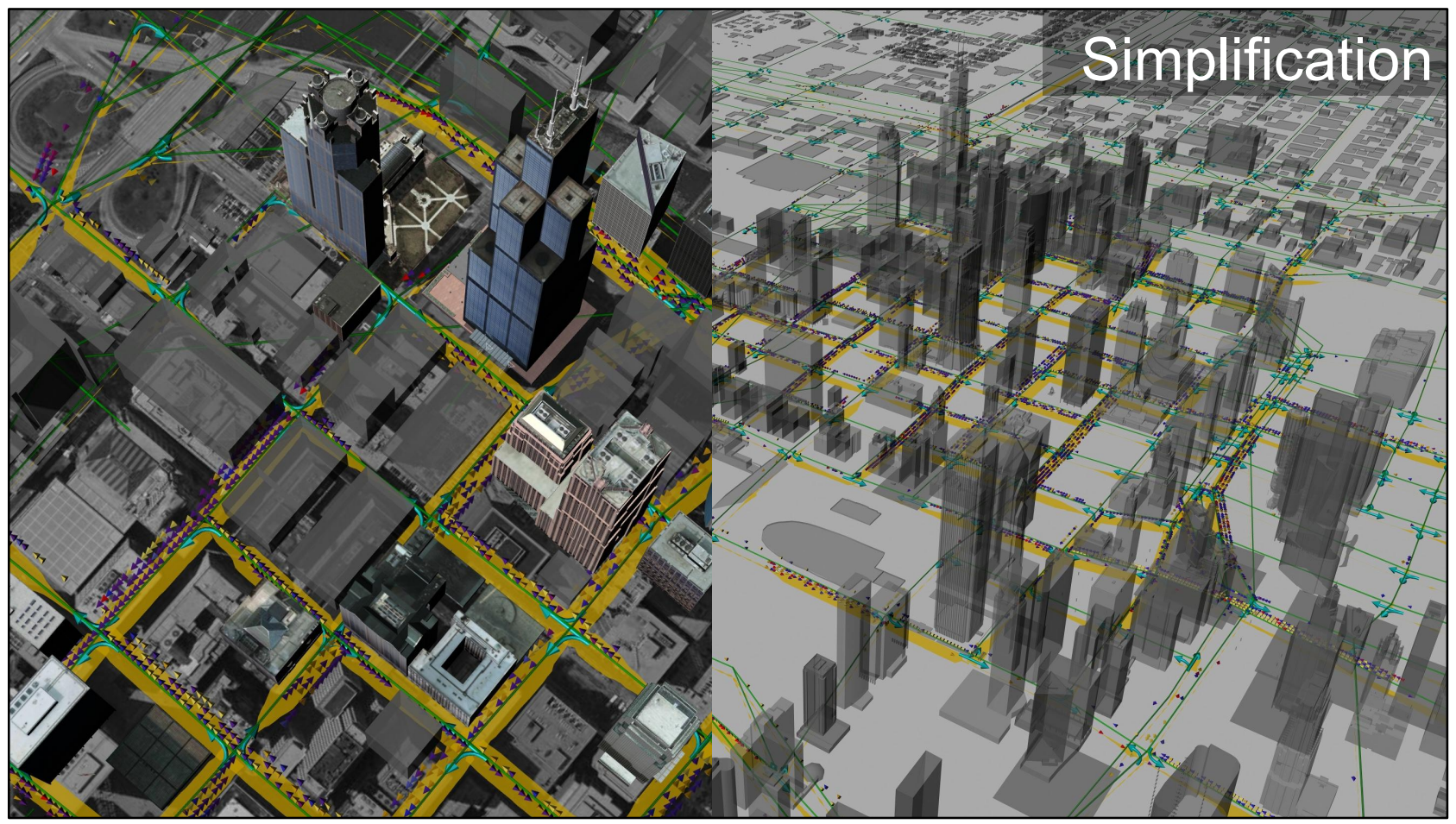

In this visualization of a computational model of road traffic in Chicago, we initially started developing the contextual environment of the city skyscrapers with opaque image textures that felt photographic. However after evaluating this approach, it became clear that the environment was overtaking the data. The final render emphasized the traffic, while the skyscrapers in the environment were made monochromatic and translucent. 


\section{Techniques: Interactive Exploration}

Interactive interrogation of a data set is often considered the strongest way for a learner to reveal features in the data and improve their metavisual capabilities.

As communicators of the data, we always start with an interactive pre-visualization of the data, though it takes significant effort. Exploring these interactive setups helps us gain a firm grasp on the science we will be required to convey. 


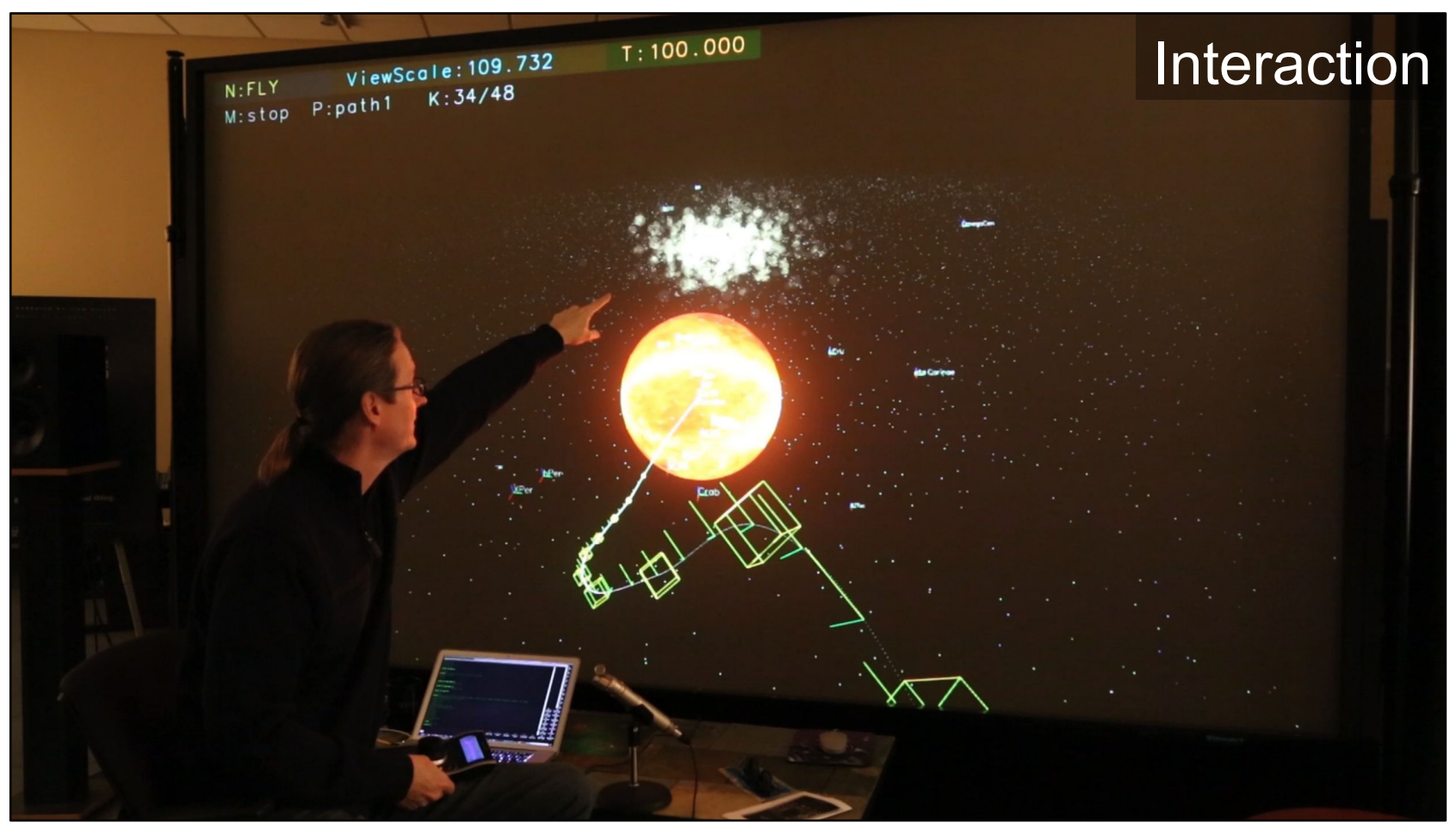

In this scene, camera choreographer Bob Patterson is investigating a scene with three embedded datasets of vastly different scales. This scene has what we call an "autoscale point", which changes the sensitivity of the camera movement as it gets closer to very tiny details, thereby giving the operator much more control. This allows a camera performance to dramatically speed up when leaving the region of interest or slow down when approaching the region of interest. This also makes multi-scale camera moves possible during a performance recording of a navigation flight. As you approach an autoscale point of interest, the interocular distance of the stereoscopic view is automatically adjusted. We create autoscale points and adjust parameters as part of the setup of a multi-scale pre-vis environment.

While interactive scene design can be useful for scientific investigation, a visualization designer needs to understand interaction design for the visualization to make sense. In many cases, untethered exploration can lead to disorientation and frustration, while a well-designed pre-rendered video may convey the concept more clearly. 


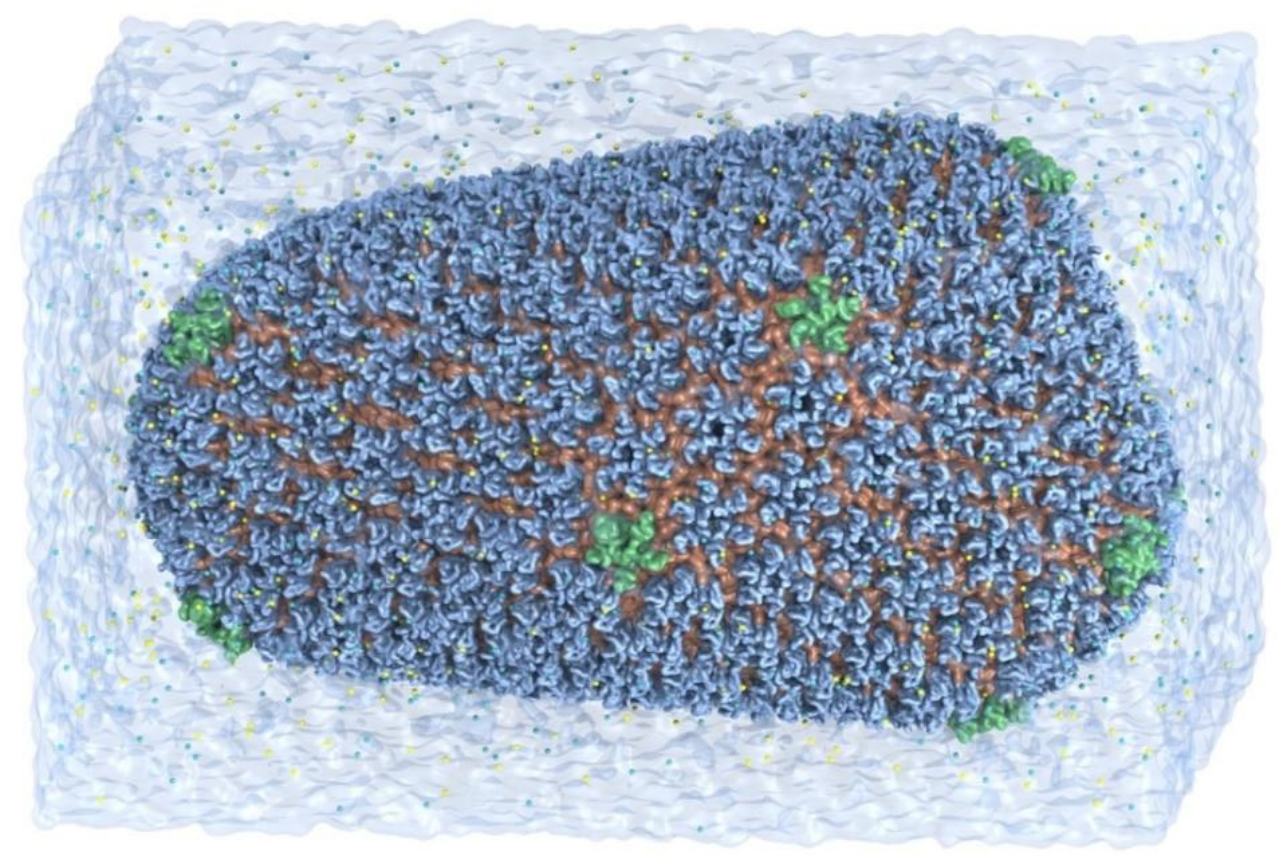

In VMD's 1.9.x versions, interactive raytracing was implemented. Ray tracing is heavily used for publication-quality images and movies in VMD because it provides high quality lighting, shadows, transparency, depth-of-field focal blur, etc. But VMD users have said that their work is difficult when the software is not interactive, and so VMD developers are now providing interactive raytracing on laptops, desktops, and remote supercomputers. 


\section{Techniques: Camera Design}

In the case of cinematic scientific visualization, allowing hundreds of audience members a simultaneous opportunity to interact with the data is simply not possible. This is why it is of paramount importance that a camera path is designed to help the audience investigate the data. 


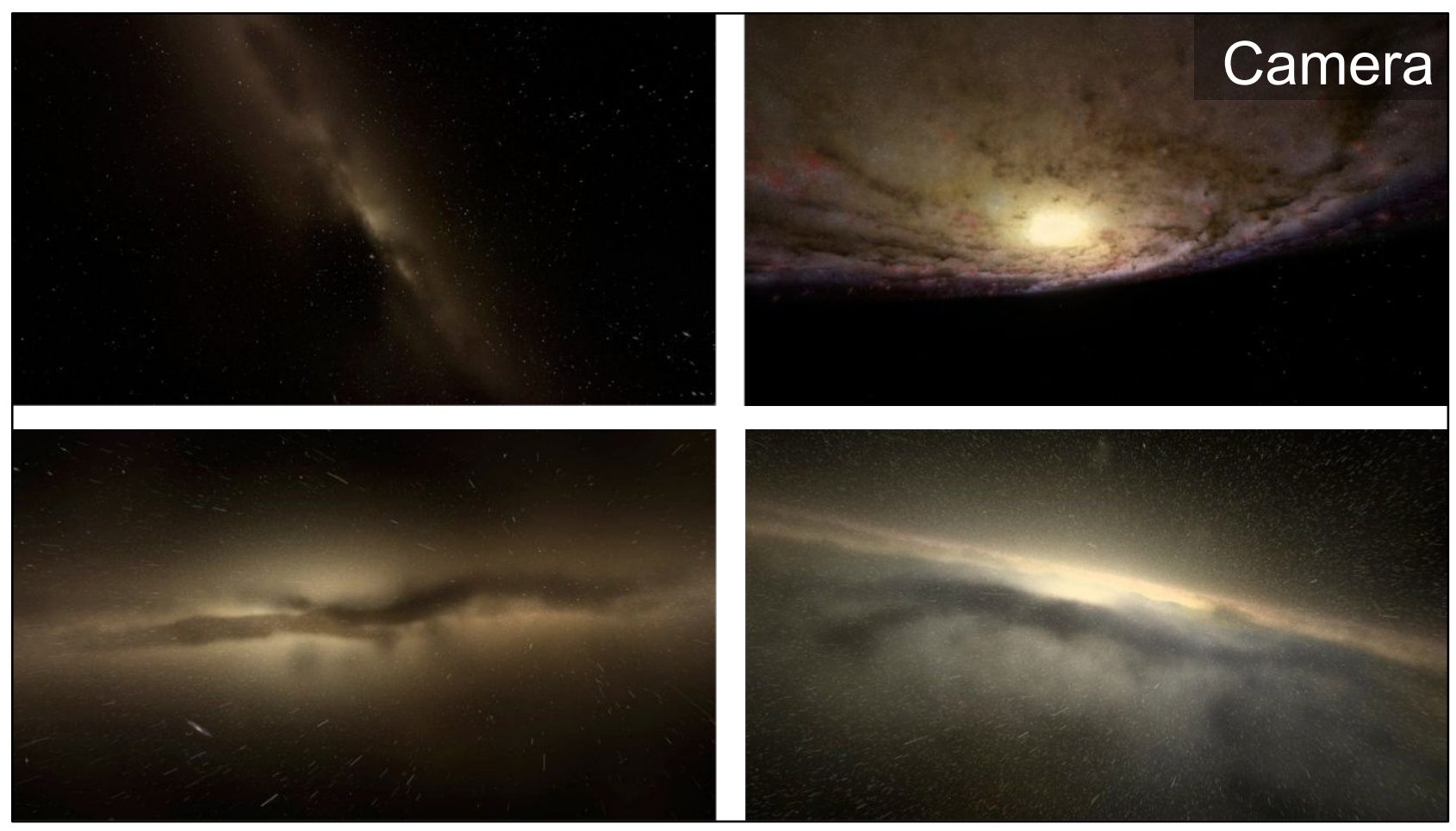

Our visualization scenes tend play out as unbroken seamless journeys to create a sense of immersion and also to help maintain context, a sense of scale and to communicate the dynamics or structure of the science. We try to create a sense of narrative with the camera move while capturing the most insightful and engaging views of the data geometry and timing. Giant screen immersive theaters are able to provide a more embodied experience and sometimes benefit from longer scenes that make you feel like you are there.

Cuts do not work well with scenes that do not have inherent directionality. Which way is "up" in space? How do we know which way we are facing, or if we are even looking at the same thing after a cut? These four images are showing the same dataset from different angles and positions, but it would be difficult for viewers to know that and to follow along if we were to cut rapidly between them. Frequent cuts also confuse our sense of depth in a stereoscopic theater. Due to their coverage of our peripheral vision, large format screens also make viewer more susceptible to motion sickness. To avoid this, camera paths must be gradual and smooth, which also helps convey large scales.

Because of this, large format screens benefit from long unbroken shots. These long shots have the added benefit of providing some breathing room between subsequent events in the simulation data. This breathing room is important to give the narrator enough time to describe the phenomenon, and to give the audience time to digest the information. 


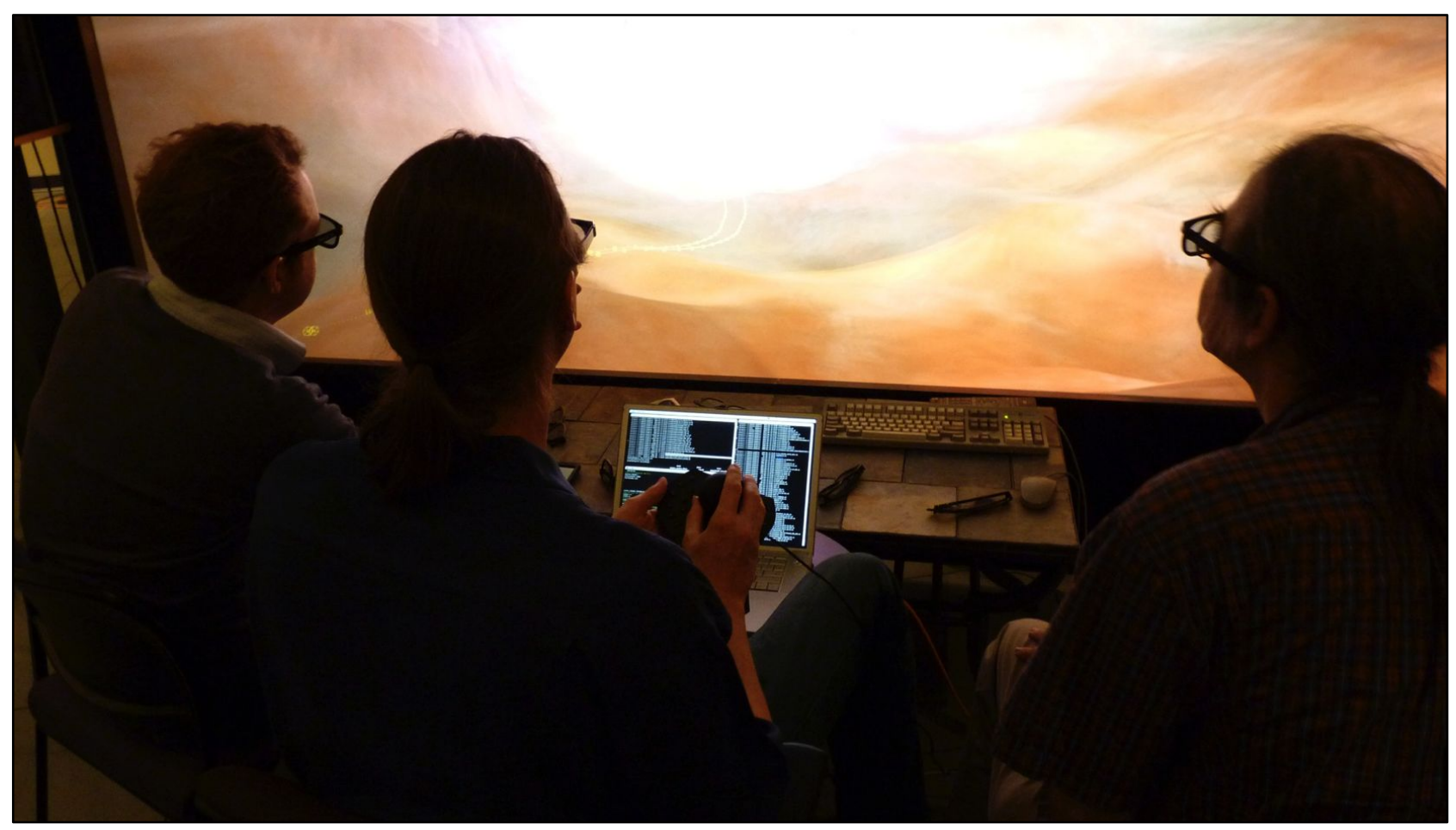

Typically AVL's lead artist and camera choreographer Bob Patterson tries to give the audience as many different views of the same object as possible, and gives them plenty of time to comprehend its shape. It's as if they are looking over the shoulder of someone else using a fully interactive camera to explore the data themselves (because that essentially is what's happening.) 


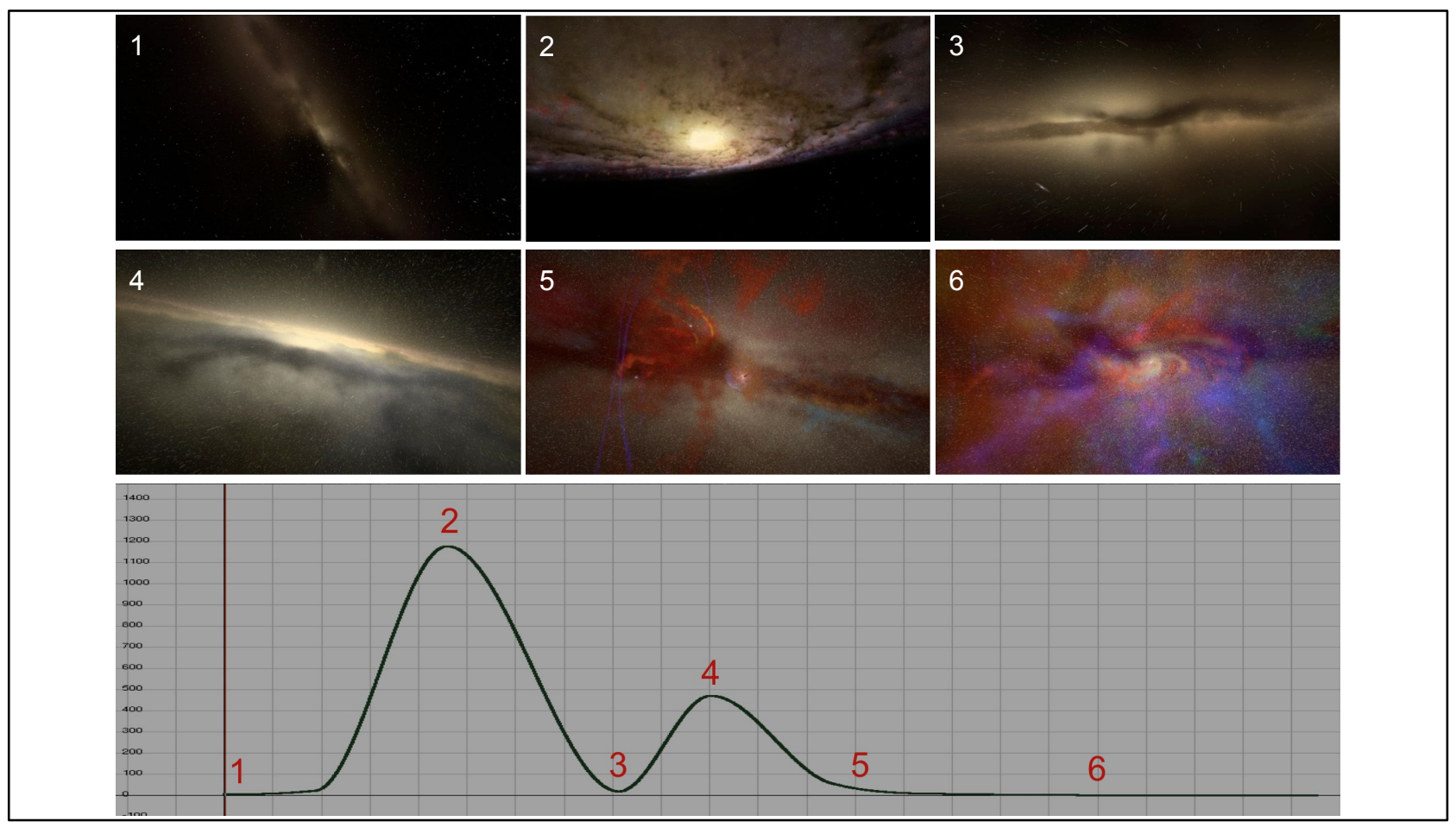

In addition to designing the camera path itself, we also consider stereoscopic design for 3D sequences. Physical sciences are often concerned with objects interacting across vastly different scales, and the ability to see these different scales at various stereoscopic depths depends on the interocular distance between the left- and right-eye cameras. 


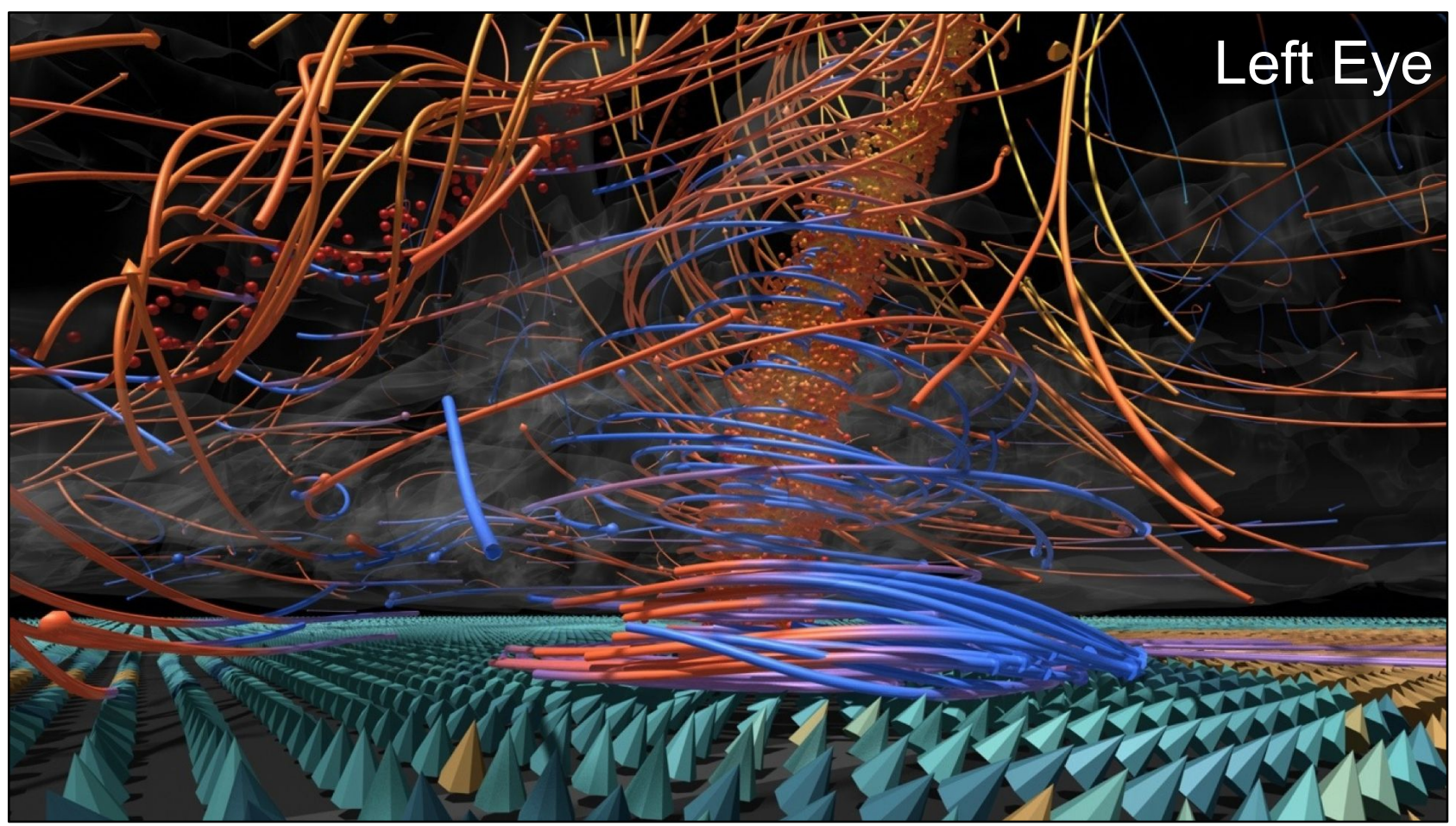

\section{LEFT EYE}

In these two views of the same scene, you can do a flicker test to see which features are to left and right of each other. This is a quick way to evaluate whether features are inside or outside of the screen plane. Typically it's recommended that the majority of the scene is kept behind the screen plane so that objects disappearing off the edge of the screen appear to go behind the image frame, instead of in front of it. 




RIGHT EYE

In these two views of the same scene, you can do a flicker test to see which features are to left and right of each other. This is a quick way to evaluate whether features are inside or outside of the screen plane. Typically it's recommended that the majority of the scene is kept behind the screen plane so that objects disappearing off the edge of the screen appear to go behind the image frame, instead of in front of it. 


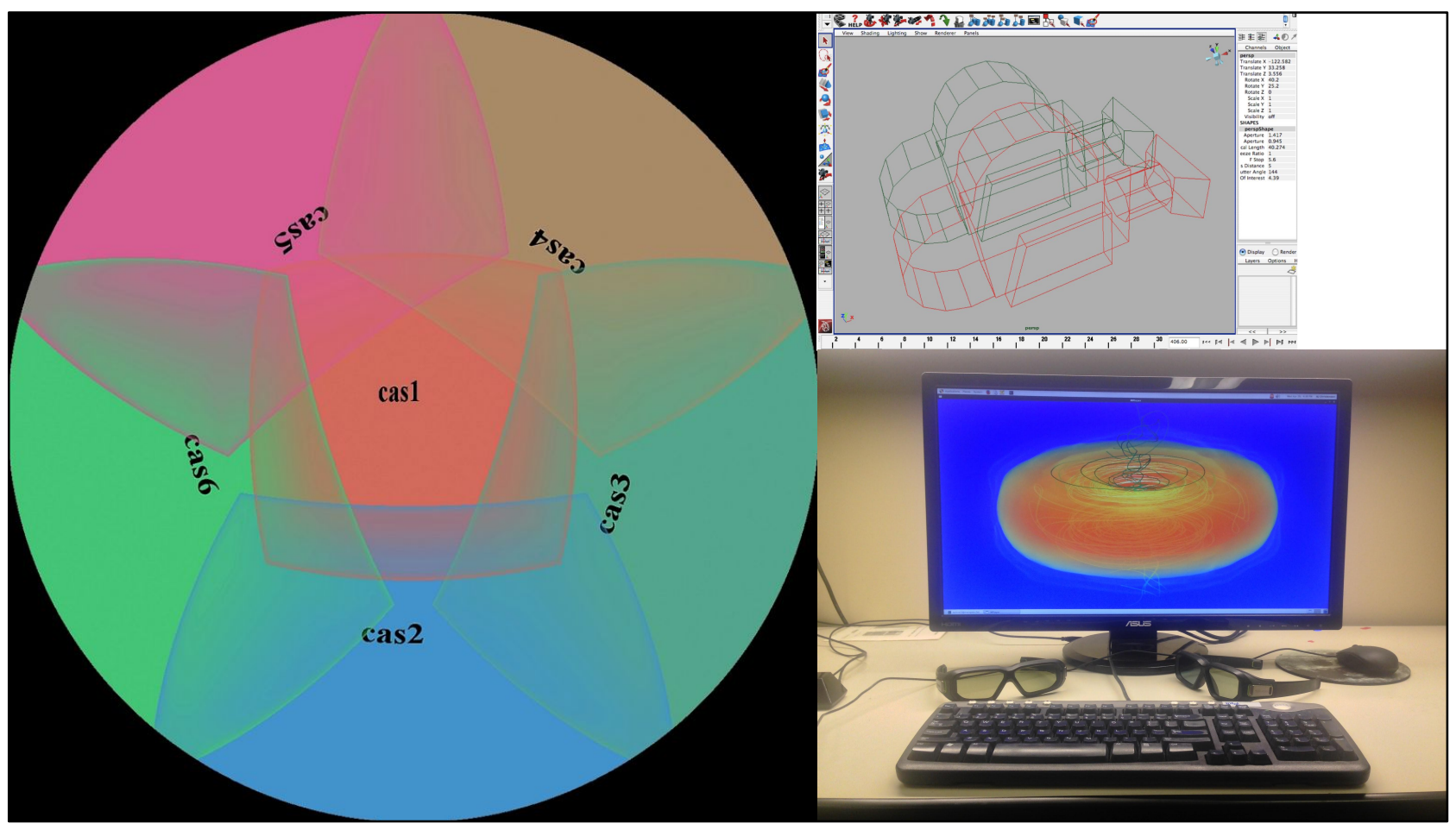

It is important to be aware of what type of screen for which you're designing a camera path and how audiences experience it. For instance, fulldome theaters often have different "sweet spots" - i.e. the area of the screen toward which the audience is predominantly facing. Dome theaters also have no upper frame edge, so to make something appear from off-screen, it must come from below the bottom frame edge.

On small screens, the camera can move faster and can cut more often. In environments where the viewer's peripheral vision is filled, it's not advisable to roll the camera too quickly, or they will lose their sense of balance.

Learning the intuition to design camera paths for various environments takes research and practice. Camera design novices are often surprised by the unintended effects of their camera moves when switching between different display environments. 


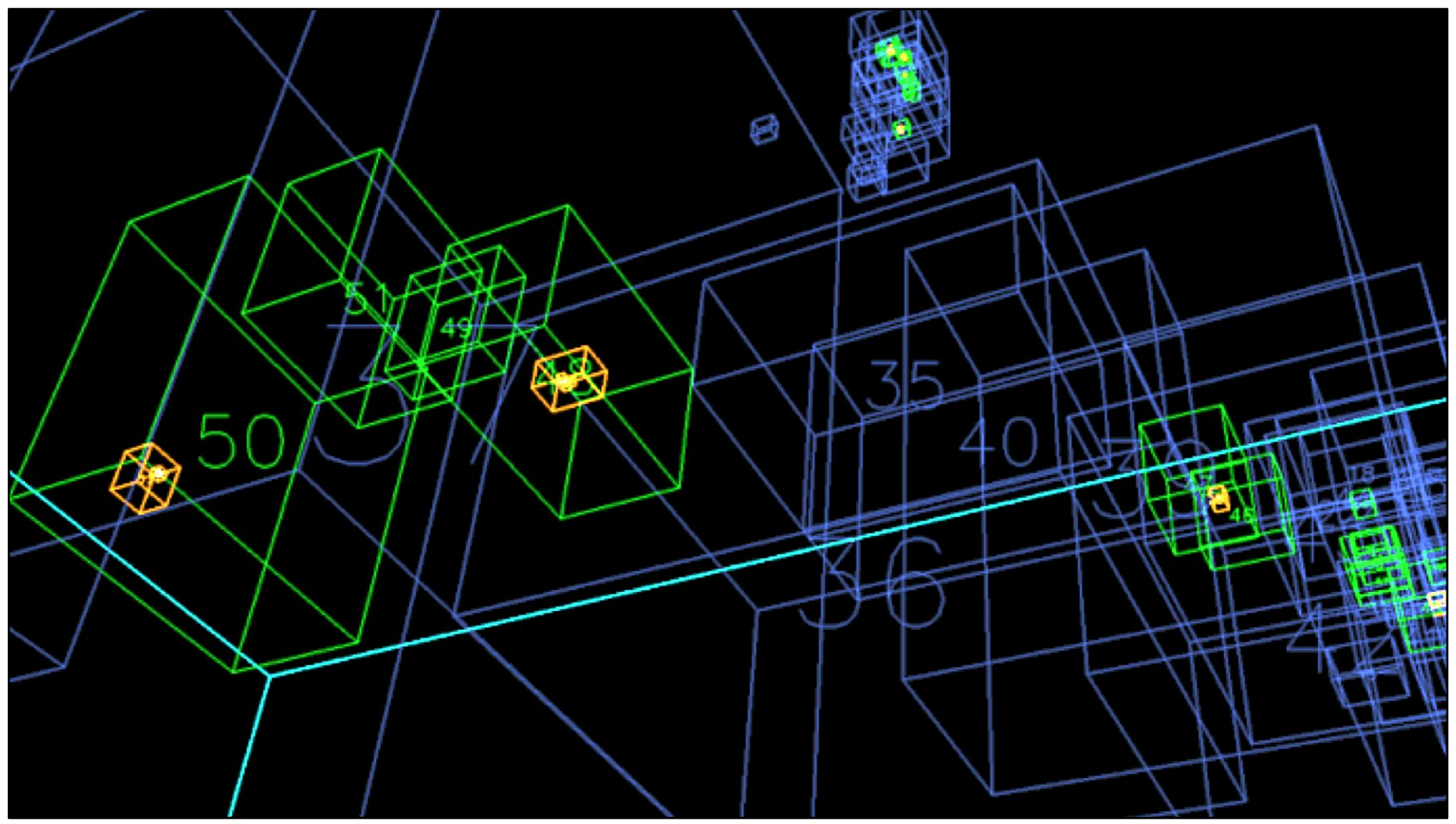

Sometimes datasets are extremely difficult to simplify for real time playback. These AMR boxes represent areas of differing resolution - where yellow boxes have the finest resolution and cyan boxes have the coarsest resolution. While getting the camera close to regions of finer resolution is generally a good idea, position and orientation are still largely guesswork.

In scenarios like this, it's important to iterate many times between fast interactive previews and slowly processed raytraced volume renders to be confident your camera design visits the most photogenic features in the simulation. 


\section{Techniques: Interpolation}

Large simulations take a lot of memory. It is impractical, and not necessarily scientifically useful, to write out many time steps.

Thus, data time steps we receive may be infrequent and/or non-linear. Scientists may write out data less frequently when there is less happening, and more frequently during exciting events.

We sometimes get simulations with less than 100 time steps, which we want to turn into a 2000 frame shot in which the simulation appears to evolve smoothly. We calculate in-between time steps, often using data attributes like velocity for more accurate interpolation. 


\section{No Interpolation}

\section{Time 60}

There are more and less accurate forms of interpolation. Linear point-to-point interpolation might be the least accurate, where B-spline or advection interpolation might be some of the more convincing methods.

Regardless of interpolation type, visualization purists often argue that interpolation of data is lying or misleading. Scientific simulation software runs calculations across incredibly complex physics formulae, and they argue that using an interpolation scheme to bypass those physical formulae can be inconsistent with the data. Furthermore, showing a simulation with more time resolution than was actually calculated can be a misrepresentation of that work to the scientific community.

Simulation credit:

Giant Impact Lunar Formation: Robin Canup, Southwest Research Institute 


\section{No Interpolation}

\section{Time 70}

However, it's apparent in this early draft of a visualization of a planetary collision that without introducing more temporal resolution, the entire event is over in a moment.

Simulation credit:

Giant Impact Lunar Formation: Robin Canup, Southwest Research Institute 


\section{No Interpolation}

\section{Time 80}

We could consider holding on a single datastep for multiple frames of the film, thereby lengthening the event. But the choppy evolution still makes it difficult to internalize all the physical interactions captured in this simulation.

Simulation credit:

Giant Impact Lunar Formation: Robin Canup, Southwest Research Institute 


\section{Interpolation}

\section{Frame 0060}

In education literature, findings indicate animations are not necessarily beneficial to learners merely because they present the information as objectively as possible. Rather, visualization designers are encouraged to play a proactive role in manipulating the way content is presented, so learners can extract thematically relevant information. If visualization designers collaborate closely with their scientific advisors, they will succeed in creating animations that are meaningful to their audience and agreeable to the domain experts.

The following interpolation test footage shows the particles in the simulation moving 10 times slower - using a fraction of their velocity vector at each frame - and providing a more natural immersive experience.

Simulation credit:

Giant Impact Lunar Formation: Robin Canup, Southwest Research Institute 


\section{Interpolation}

\section{Frame 0061}

Simulation credit:

Giant Impact Lunar Formation: Robin Canup, Southwest Research Institute 


\section{Interpolation}

\section{Frame 0062}

Simulation credit:

Giant Impact Lunar Formation: Robin Canup, Southwest Research Institute 


\section{Interpolation}

\section{Frame 0063}

Simulation credit:

Giant Impact Lunar Formation: Robin Canup, Southwest Research Institute 


\section{Interpolation}

\section{Frame 0064}

Simulation credit:

Giant Impact Lunar Formation: Robin Canup, Southwest Research Institute 


\section{Interpolation}

\section{Frame 0065}

Simulation credit:

Giant Impact Lunar Formation: Robin Canup, Southwest Research Institute 


\section{Interpolation}

\section{Frame 0066}

Simulation credit:

Giant Impact Lunar Formation: Robin Canup, Southwest Research Institute 


\section{Interpolation}

\section{Frame 0067}

Simulation credit:

Giant Impact Lunar Formation: Robin Canup, Southwest Research Institute 


\section{Interpolation}

\section{Frame 0068}

Simulation credit:

Giant Impact Lunar Formation: Robin Canup, Southwest Research Institute 


\section{Interpolation}

\section{Frame 0069}

Simulation credit:

Giant Impact Lunar Formation: Robin Canup, Southwest Research Institute 


\section{Interpolation}

\section{Frame 0070}

Simulation credit:

Giant Impact Lunar Formation: Robin Canup, Southwest Research Institute 


\section{Techniques: Registration}

In order to provide as much context as possible for a visualization, our team often integrates many different simulations and data sources. This task is far from trivial as different scientists use different units of measurement, data translation and computer graphics artifacts can appear, and in many cases it requires our team of artists and technologists to domain science calculations. 


\section{Registration}

In this visualization of a tornadic storm that hit Joplin Missouri in 2011, an important element of the story the data was telling was about the real city and human lives that were affected. It was important to ensure that the tornado was placed in the correct geographic position.

Image Credits:

Joplin 2011 Storm Simulation: Brian Jewett and Kevin Van Leer, University of Illinois at Urbana-Champaign GIS data: Johnathan Rush, Junjun Yin, Aiman Soliman, Dandong Yin, and Shaowen Wang, CyberGIS Center for Advanced Digital and Spatial Studies, Funded in part by the National Science Foundation Awards: 1047916 and 1443080

Visualization by: Advanced Visualization Lab, National Center for Supercomputing Applications, University of Illinois at Urbana-Champaign, Donna Cox, Kalina Borkiewicz, Jeff Carpenter, AJ Christensen, Stuart Levy, Robert Patterson, funded in part by: National Science Foundation Award ACl-1445176, The Centrality of Advance Digitally Enabled Science: CADENS 


\section{Storm}

This is what the storm simulation looked like on a context-free black background.

Image Credits:

Joplin 2011 Storm Simulation: Brian Jewett and Kevin Van Leer, University of Illinois at Urbana-Champaign Visualization by: Advanced Visualization Lab, National Center for Supercomputing Applications, University of Illinois at Urbana-Champaign, Donna Cox, Kalina Borkiewicz, Jeff Carpenter, AJ Christensen, Stuart Levy, Robert Patterson, funded in part by: National Science Foundation Award ACl-1445176, The Centrality of Advance Digitally Enabled Science: CADENS 


\section{Terrain}

We worked with a Geographical Information Systems (GIS) expert to find the highest-resolution map of the terrain that was available, and then registered it to the coordinate system of the storm simulation.

Image Credits:

GIS data: Johnathan Rush, Junjun Yin, Aiman Soliman, Dandong Yin, and Shaowen Wang, CyberGIS Center for Advanced Digital and Spatial Studies, Funded in part by the National Science Foundation Awards: 1047916 and 1443080

Visualization by: Advanced Visualization Lab, National Center for Supercomputing Applications, University of Illinois at Urbana-Champaign, Donna Cox, Kalina Borkiewicz, Jeff Carpenter, AJ Christensen, Stuart Levy, Robert Patterson, funded in part by: National Science Foundation Award ACl-1445176, The Centrality of Advance Digitally Enabled Science: CADENS 


\section{Registration}

The final scene not only includes the two sources of information registered to fit together, but uses seamless lighting and depth fog to unify the two data sources into one coherent picture.

Image Credits:

Joplin 2011 Storm Simulation: Brian Jewett and Kevin Van Leer, University of Illinois at Urbana-Champaign GIS data: Johnathan Rush, Junjun Yin, Aiman Soliman, Dandong Yin, and Shaowen Wang, CyberGIS Center for Advanced Digital and Spatial Studies, Funded in part by the National Science Foundation Awards: 1047916 and 1443080

Visualization by: Advanced Visualization Lab, National Center for Supercomputing Applications, University of Illinois at Urbana-Champaign, Donna Cox, Kalina Borkiewicz, Jeff Carpenter, AJ Christensen, Stuart Levy, Robert Patterson, funded in part by: National Science Foundation Award ACl-1445176, The Centrality of Advance Digitally Enabled Science: CADENS 


\section{Registration}

In a digital fulldome show we worked on with the California Academy of Sciences in San Francisco, we fly faster than the speed of light through a virtual Milky Way galaxy until we arrive at what first appears to be a small bright dot - an entire forming planetary system. 


\section{Molecular Cloud}

Because this so-called protoplanetary disk was at such a vastly different scale from the galaxy we were flying through, we thought it would be helpful to have an intermediary scale. We got lucky in discovering a scientist willing to share this molecular cloud simulation, essentially a nebula in which the protoplanetary disk could be born. We were able to render this adaptive mesh refinement (AMR) data with our custom renderer "Amore". 


\section{Extraction}

We registered the protoplanetary disk with the molecular cloud in Maya using a custom data processing plugin. We extracted a small local part of the molecular cloud to render around the protoplanetary disk in Maya as well to help with integration. 


\section{Registration}

We then registered both of those datasets inside our virtual Milky Way model, which was rendered with a custom tool called "Star Renderer". We combined the layers in a compositing tool for the final integrated images. 


\section{Techniques: Compositing}

Compositing allows us to combine data rendered from different software to seamlessly integrate our contextual elements. We also incorporate compositing as part of the standard VFX pipeline to make our images beautiful, artifact-free, and focused. 


\section{Compositing}

In this breakdown of a visualization composite done in (the now-unsupported) compositing software Shake for the IMAX film "Hubble 3D", we create an environment out of the Orion Nebula. The geometric surface was provided to us by Hubble scientists at the Space Telescope Science Institute, sculpted from an 18,000 square pixel image mosaic of Hubble photographs. 
AVL programmer Alex Betts wrote a plugin for Maya that would splat geometry into a volume, which gave the nebula its cloudy look. 


\section{Compositing}

Blue clouds which we refer to as "the veil", and which the scientists know are separate from the other clouds, were separated from the original Hubble imagery and brought back into the scene as a dome over the environment. 
Then small objects like stars, proplyds, and bowshocks which were removed from the original Hubble image are returned to the scene in hovering 3D positions. 


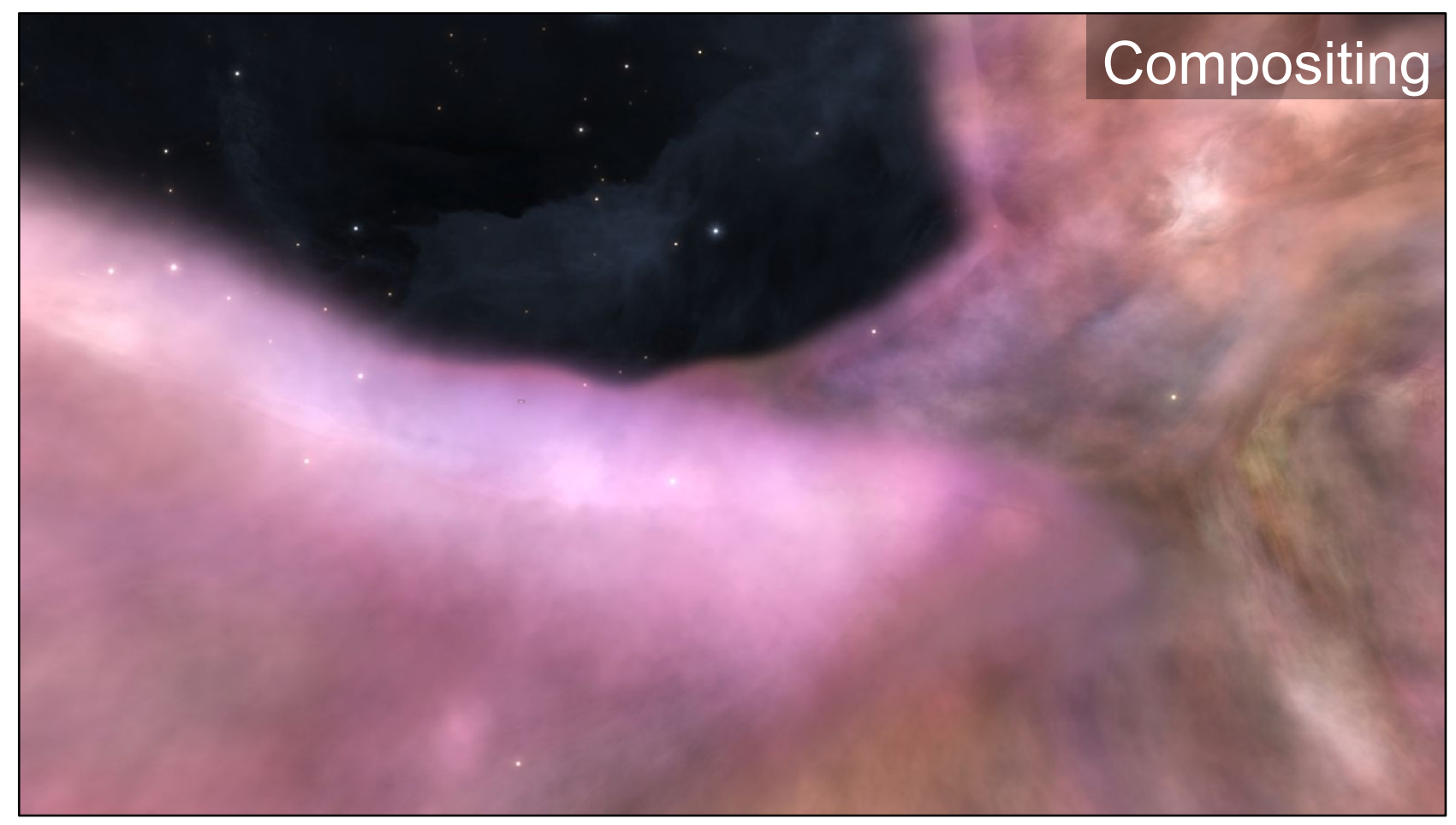




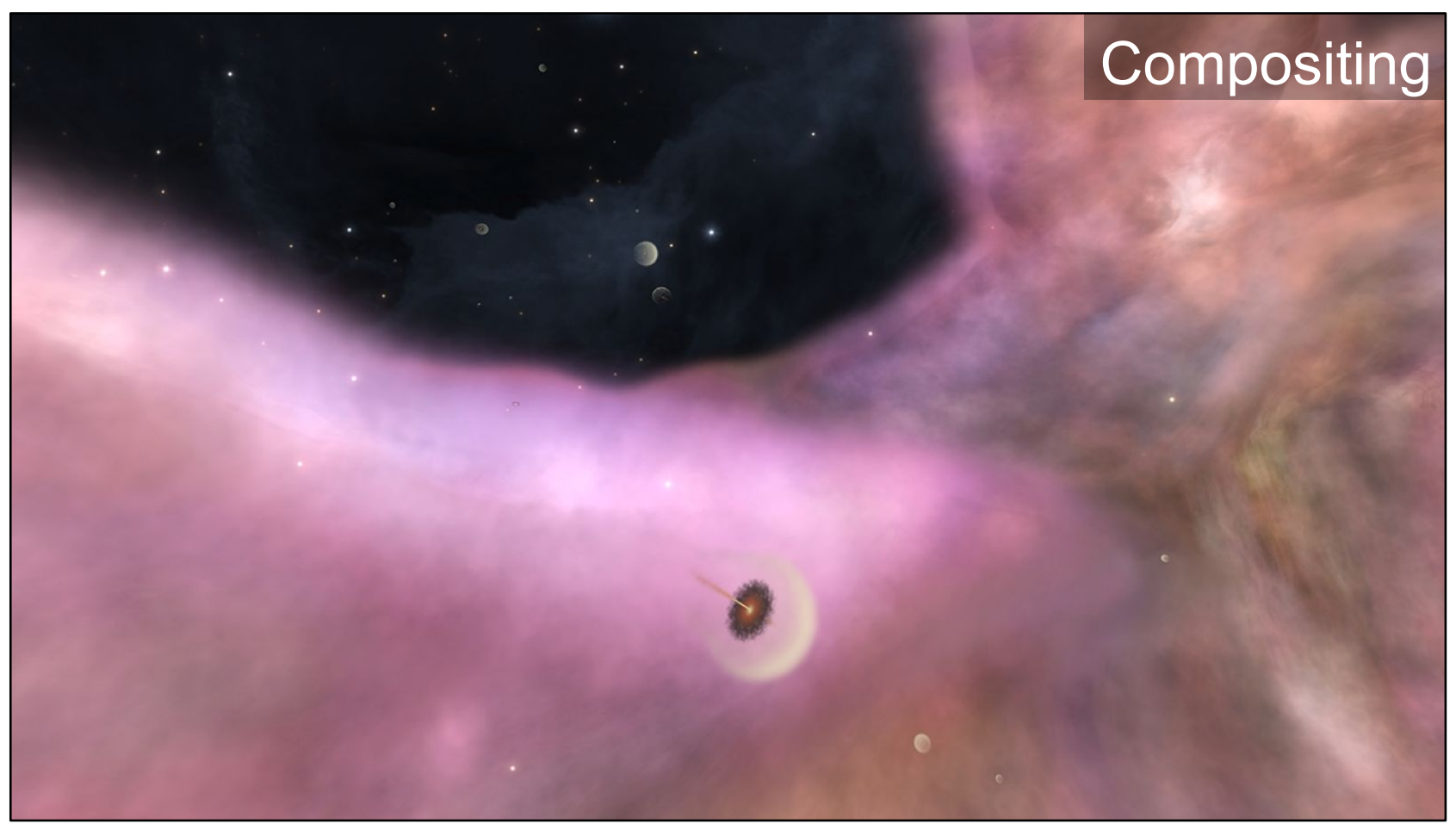




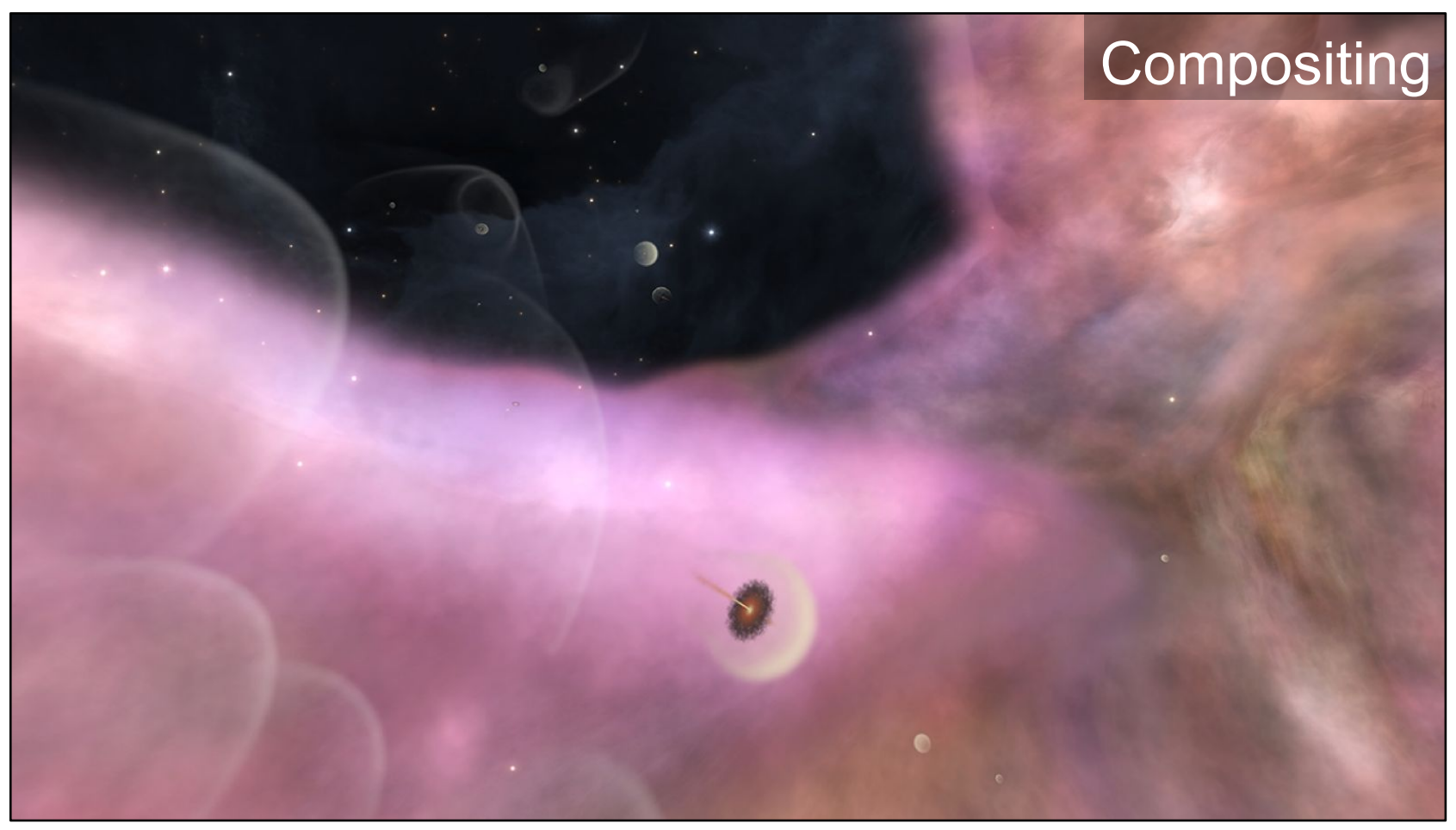




\section{Final Render}

And finally we do image grading to make the imagery vibrant by using the full range of pixel brightness and color representation. 


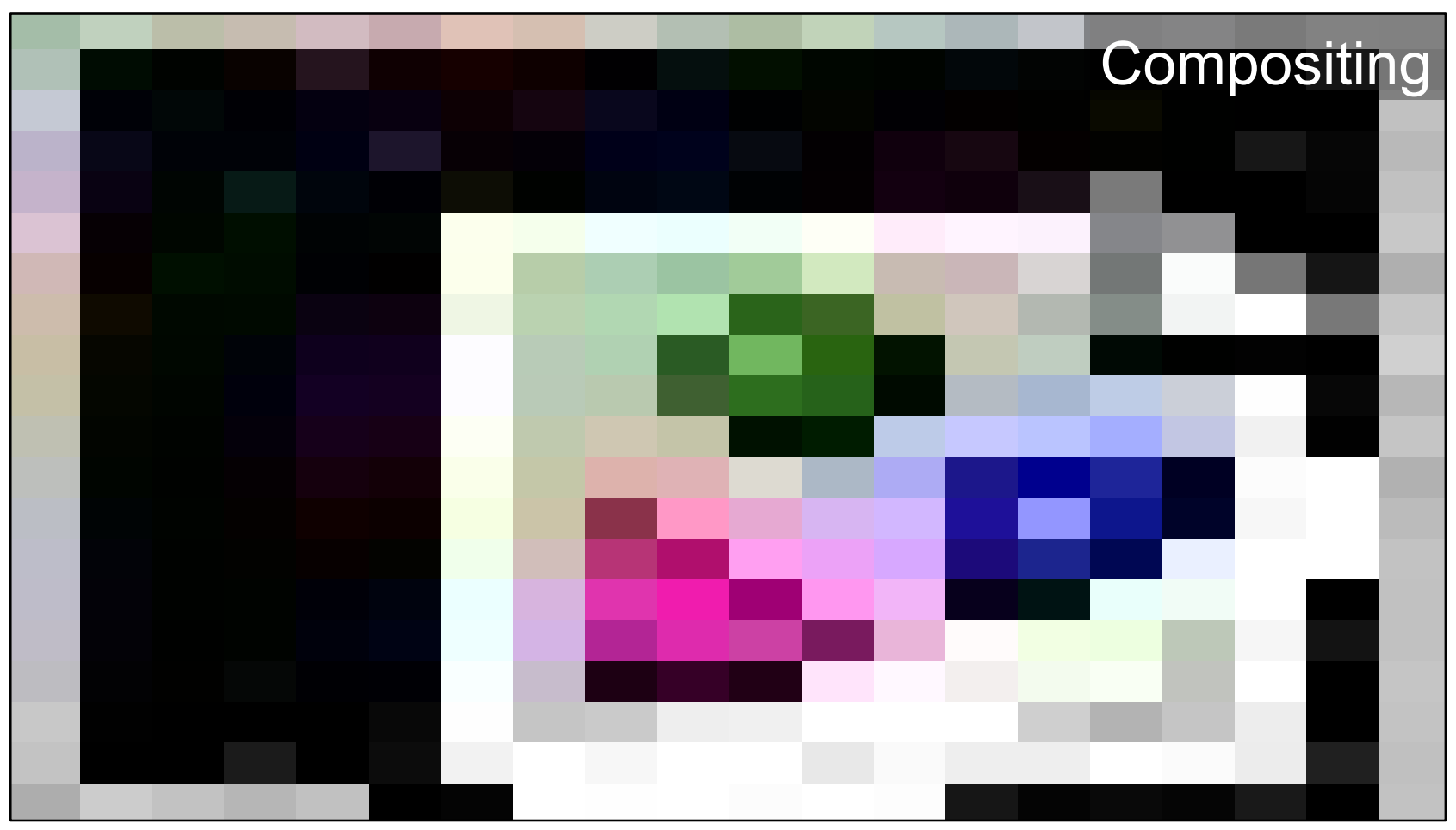

After updating our pipeline to rely more heavily on multi-volume rendering in Houdini, which seems to manage memory of large datasets well, we don't always need to rely on the compositor solely to combine datasets. However, as this Nuke composite demonstrates, combining datasets in a beautiful way often relies on 2D image manipulation.

This visualization has us looking through the surface of the sun, into the interior. This required combining several different datasets, each from a different team of scientists. The main features we are trying to show here are the "bananas" (strong distinct vortices) rolling across the sun's equator, on the inside of the sun, but in order to do this effectively, we also need to show the exterior of the sun, as well as other features. Showing just the interior may make sense to a specialist, but to a general audience, we need to show this $x$-ray vision in order to add context and have the whole picture make sense.

Image Credits:

"Solar Superstorms": A full-dome production by NCSA, University of Illinois, Thomas Lucas Productions, Spitz Creative Media

"Solar Dynamo / Solar Interior" simulation by: Juri Toomre (JILA, University of Colorado Boulder), Mark Miesch (High Altitude Observatory, National Center for Atmospheric Research), Nicholas Nelson (Los Alamos National Laboratory), Allan Sacha Brun (Commissariat à l'énergie atomique et aux énergies alternatives, Saclay, France), Benjamin Brown (Laboratory for Atmospheric and Space Physics, University of Colorado Boulder)

"Active Region Scale Flux Emergence on the Sun" simulation by: Matthias Rempel (High Altitude Observatory, National Center for Atmospheric Research), M.C.M. Cheung (Lockheed Martin Solar and Astrophysics Lab) SDO Data: Steele Hill (SOHO/STEREO/SDO Media Specialist, NASA Goddard Space Flight Center)

Visualization by: Advanced Visualization Lab, National Center for Supercomputing Applications, University of Illinois at Urbana-Champaign, Donna Cox, Kalina Borkiewicz, Jeff Carpenter, AJ Christensen, Stuart Levy, Robert Patterson Funded in part by: National Science Foundation Award ACl-1445176, The Centrality of Advance Digitally Enabled Science: CADENS 
We begin by putting the whole sun in the correct context - the background sky image is what we would see when looking in the direction of the sun, from the Earth. 




Around the rim of the sun, we used real time lapse imagery from NASA's Solar Dynamics Observatory.

Credit:

Solar Dynamics Observational Data: Steele Hill (SOHO/STEREO/SDO Media Specialist, NASA Goddard Space Flight Center) 


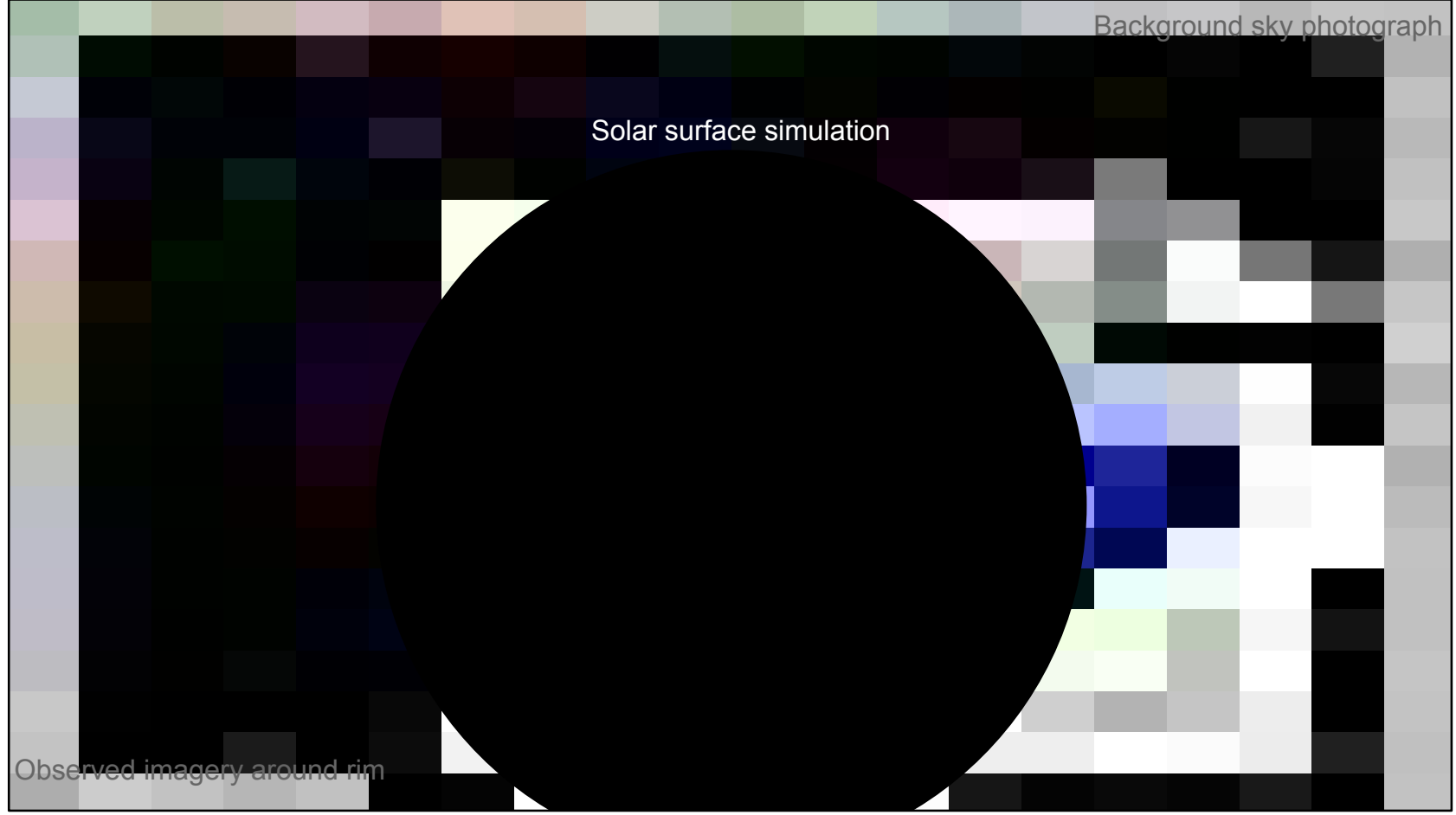

The sun's surface was one of the supercomputer simulations in this sequence. The simulation came to us as a grayscale $2 \mathrm{D}$ image, which covered a small patch of the sun's surface. We colored the image and gave it a height map based on the grayscale values. We tiled the image on a sphere, and staggered the timing of each tile in order to not have obvious repetition, smoothing between the tiles.

Credit:

"Active Region Scale Flux Emergence on the Sun" simulation: Matthias Rempel (High Altitude Observatory, National Center for Atmospheric Research), M.C.M. Cheung (Lockheed Martin Solar and Astrophysics Lab)

Spatial Scale: $150,000 \times 75,000 \mathrm{~km}$

Time Scale: 140 hours

Resolution: 1536 x 768 pixels

Data Size: 16,800 timesteps, 600GB 


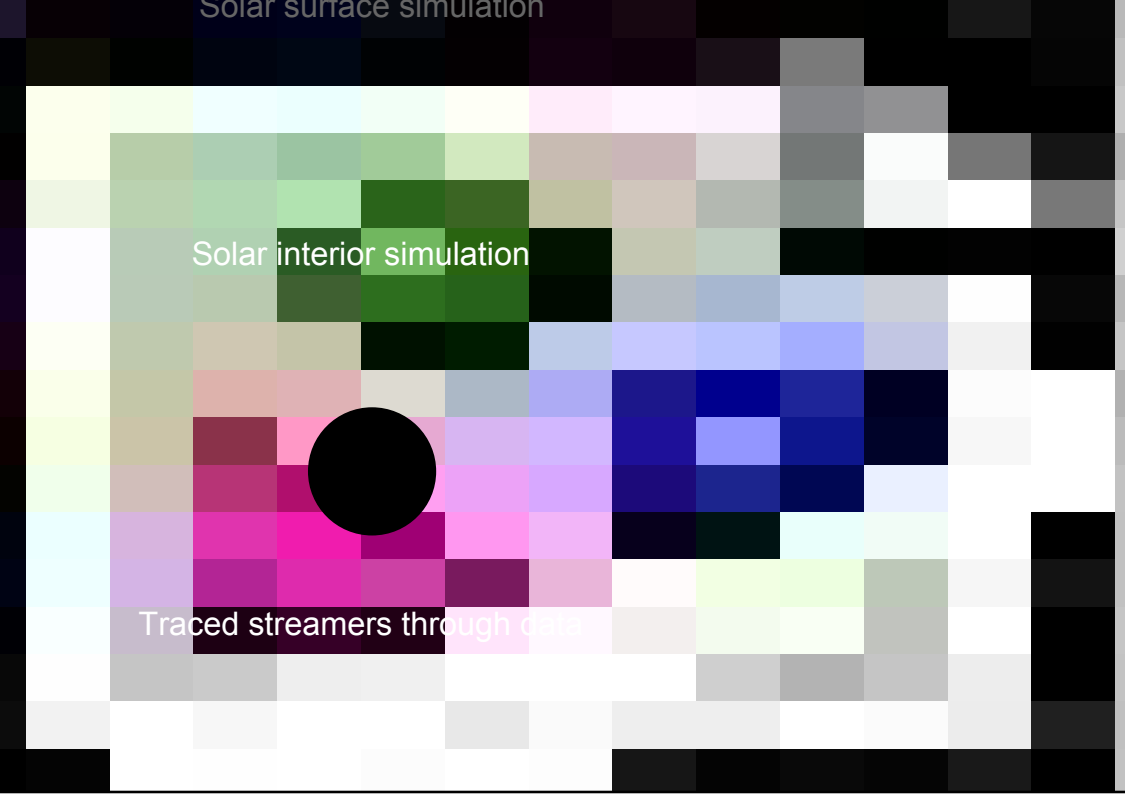

The interior simulation showed the "bananas", but their rolling motion wasn't immediately apparent in the volumetric representation. So, we advected streamers through the vorticity field in the data that helped give definition to each banana.

\section{Credit:}

"Solar Dynamo / Solar Interior" simulation by: Juri Toomre (JILA, University of Colorado Boulder), Mark Miesch (High Altitude Observatory, National Center for Atmospheric Research), Nicholas Nelson (Los Alamos National Laboratory), Allan Sacha Brun (Commissariat à l'énergie atomique et aux énergies alternatives, Saclay, France), Benjamin Brown (Laboratory for Atmospheric and Space Physics, University of Colorado Boulder)

\section{Spatial Scale: $71 \%-98 \%$ solar radius}

Time scale: 31 days

Resolution: $193 \mathrm{R} \times 512 \theta \times 1024 \Phi$

Data Size: 1511 timesteps, 3.4TB

Computed at: Laboratory for Computational Dynamics, University of Colorado, Boulder and Pleiades, NASA Advanced Supercomputing Division 


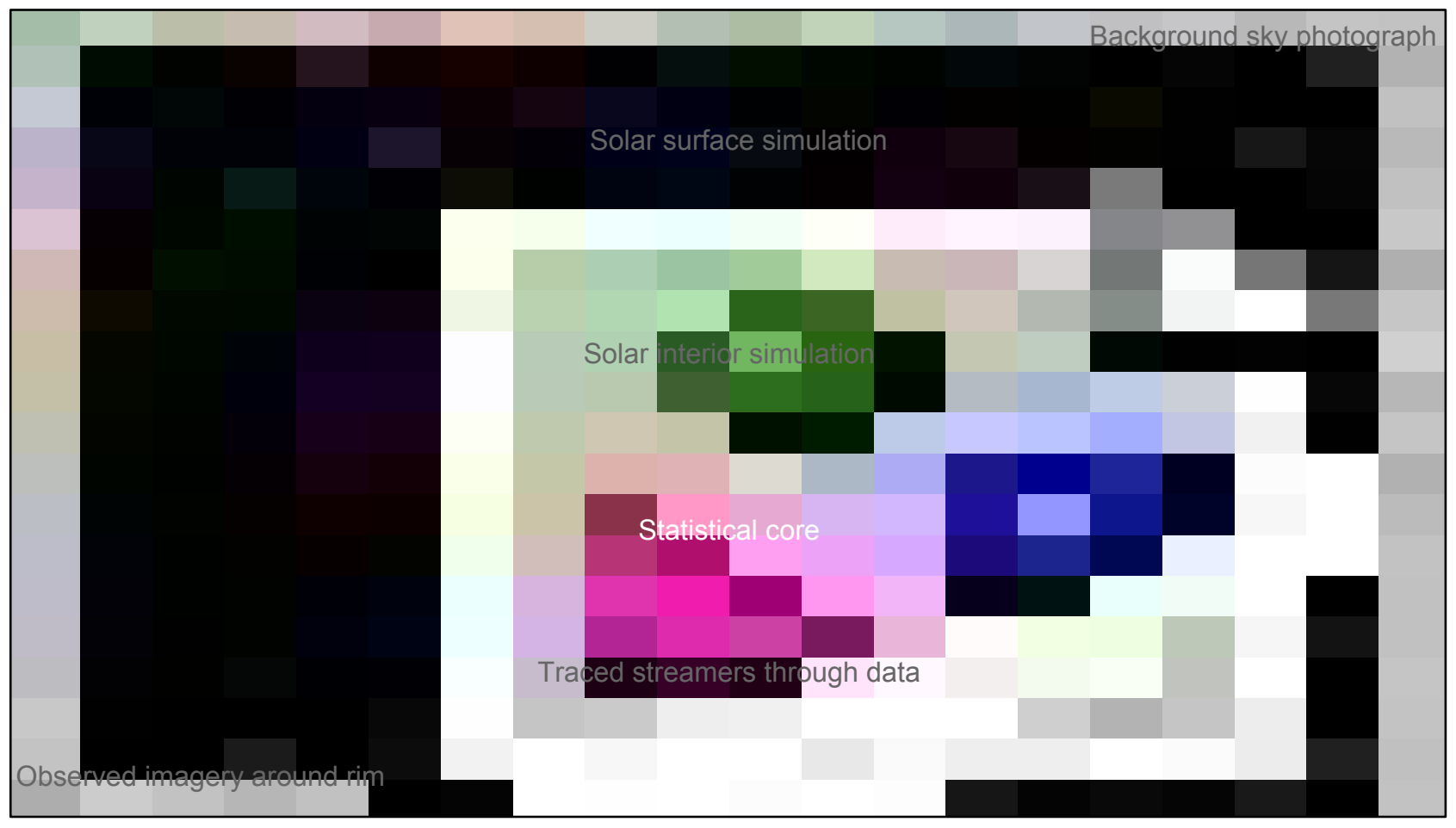

The interior simulation covered $71-98 \%$ of the sun's interior. To fill in the very interior, we added a statistical representation of the sun's core.

Image Credits:

"Solar Superstorms": A full-dome production by NCSA, University of Illinois, Thomas Lucas Productions, Spitz

Creative Media

"Solar Dynamo / Solar Interior" simulation by: Juri Toomre (JILA, University of Colorado Boulder), Mark Miesch (High Altitude Observatory, National Center for Atmospheric Research), Nicholas Nelson (Los Alamos National Laboratory), Allan Sacha Brun (Commissariat à l'énergie atomique et aux énergies alternatives, Saclay, France), Benjamin Brown (Laboratory for Atmospheric and Space Physics, University of Colorado Boulder)

"Active Region Scale Flux Emergence on the Sun" simulation: Matthias Rempel (High Altitude Observatory, National Center for Atmospheric Research), M.C.M. Cheung (Lockheed Martin Solar and Astrophysics Lab)

Solar Dynamics Observational Data: Steele Hill (SOHO/STEREO/SDO Media Specialist, NASA Goddard Space Flight Center)

Visualization by: Advanced Visualization Lab, National Center for Supercomputing Applications, University of Illinois at Urbana-Champaign, Donna Cox, Kalina Borkiewicz, Jeff Carpenter, AJ Christensen, Stuart Levy, Robert Patterson Funded in part by: National Science Foundation Award ACl-1445176, The Centrality of Advance Digitally Enabled Science: CADENS 


\section{Validation and Interpretation}

It's important when communicating science to broad audiences that we ensure our work is connecting with them, and that they are understanding the learning objectives laid out by domain experts and show producers. 


\section{Visualization can be Misleading}

At some level, all visualization is tainted by the subjective touch of its designers. We make great efforts to confirm we are not implying incorrect science.

Of course, with any powerful means of communication comes those who will abuse it. Everyday we become more and more aware of how imagery can be used to fool us. Social media, political publications, and television news are frequently guilty of presenting data in ways that are misleading in order to sell a perspective. 


\section{Rating}

\section{Misleading}

60

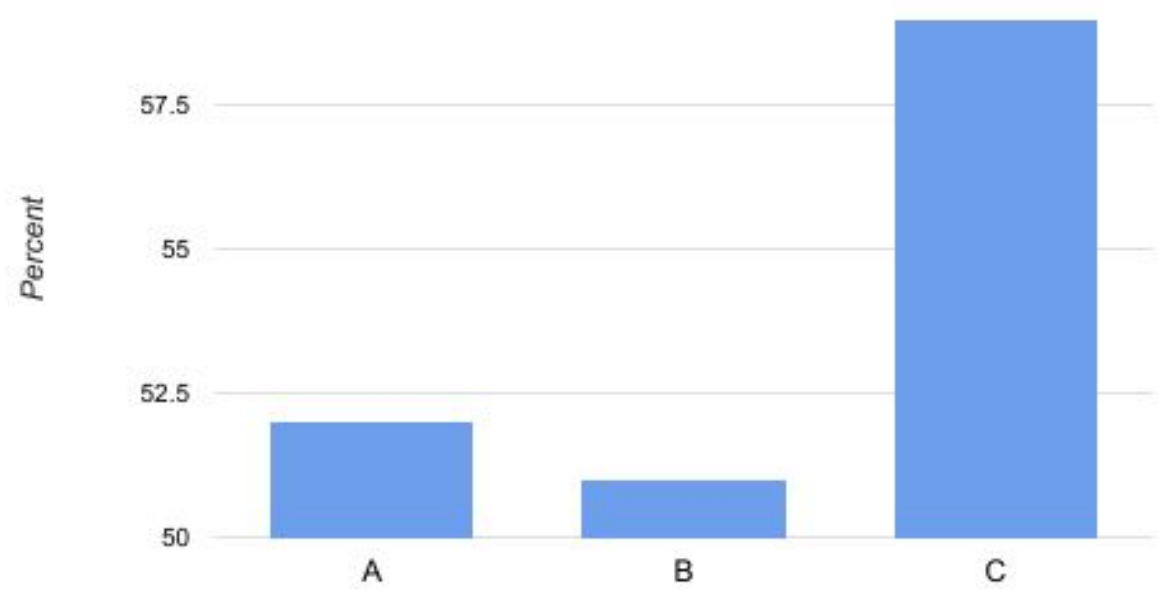

Subject

This graph is an example of how data presentation can be misleading. Just by glancing at this graph, we can assume that $C$ is much better than $A$ and $B$. We may not notice that the $y$-axis doesn't start at 0 , and shows a very small range. 


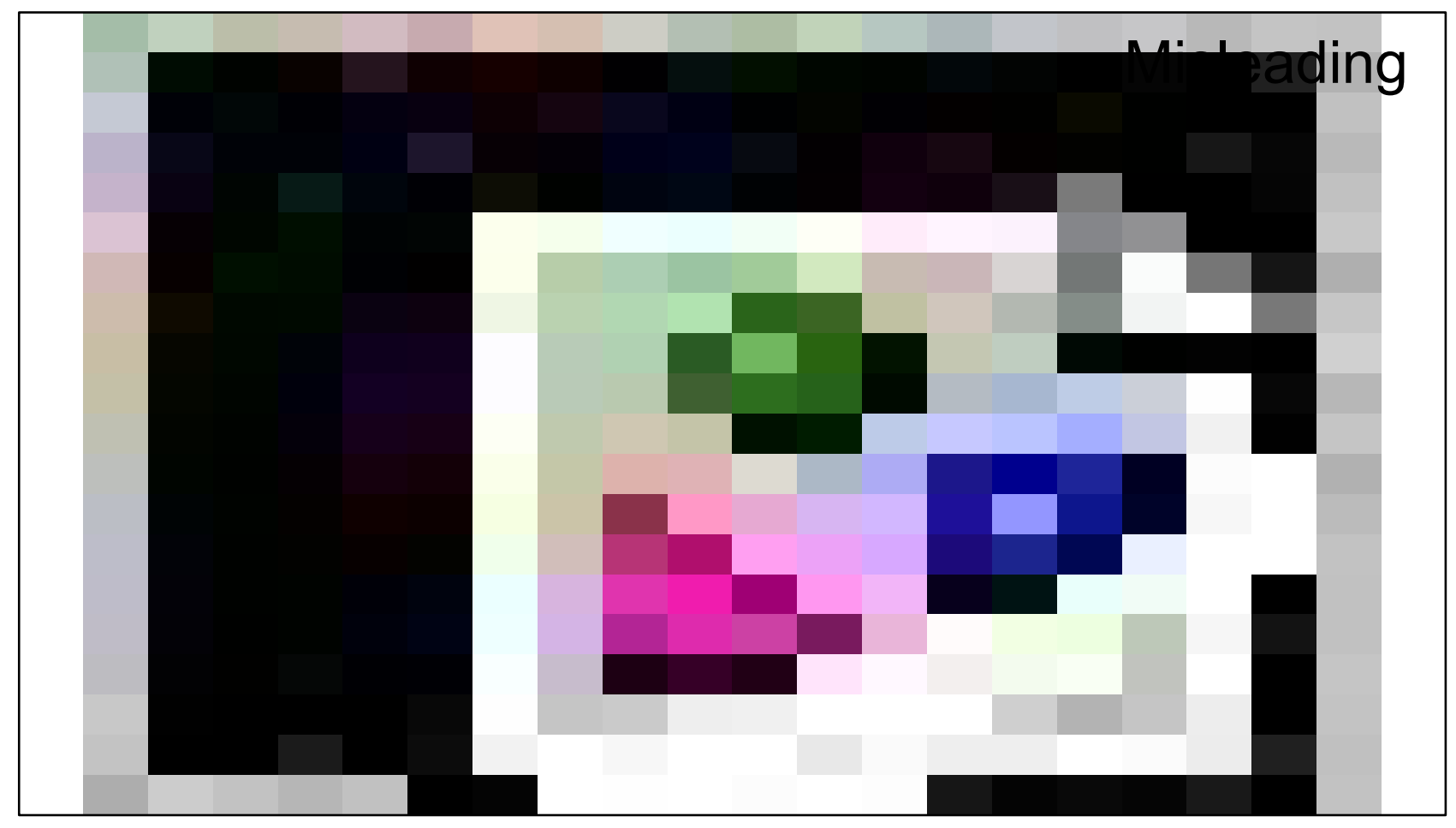

If we rescale the $y$-axis to go from $0-100 \%$, we get a different picture: $A, B$, and $C$ are about equal. 


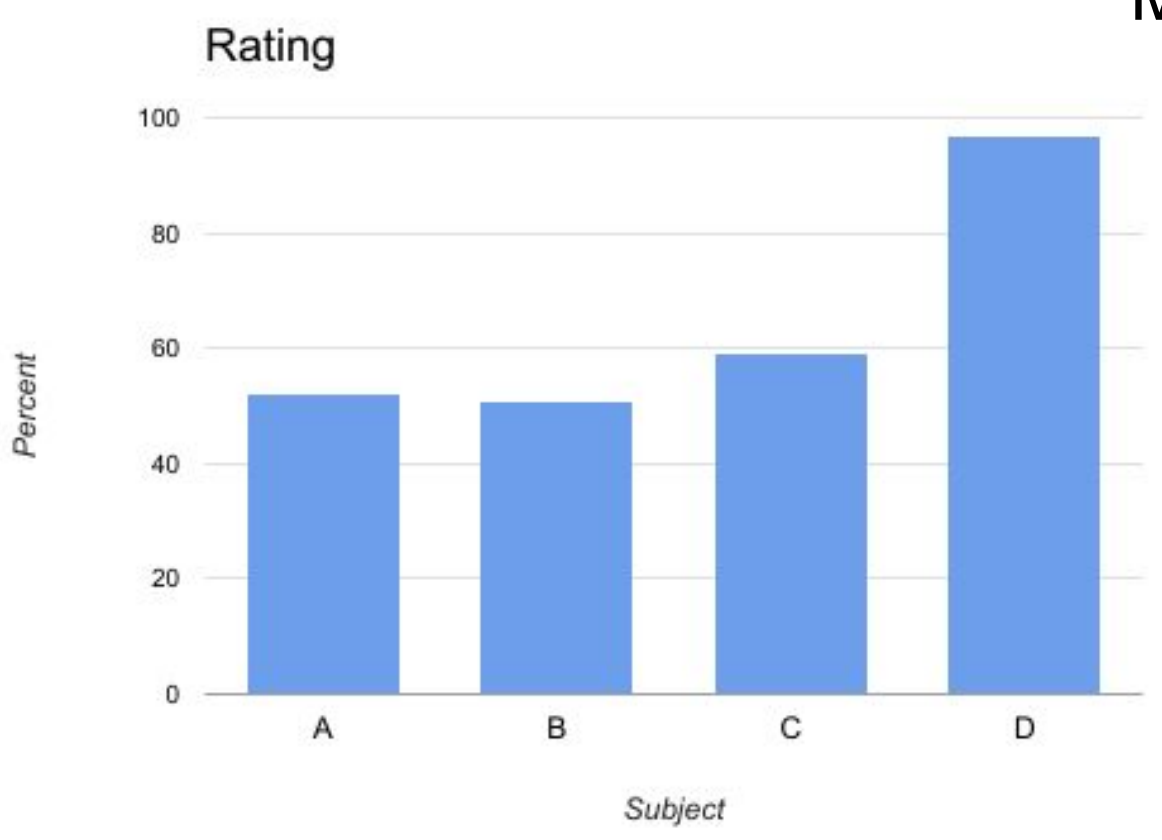

But, perhaps we were just looking at a subset of the full dataset. There is actually another subject, $D$, which has a much higher rating than $A, B$, and $C$. 


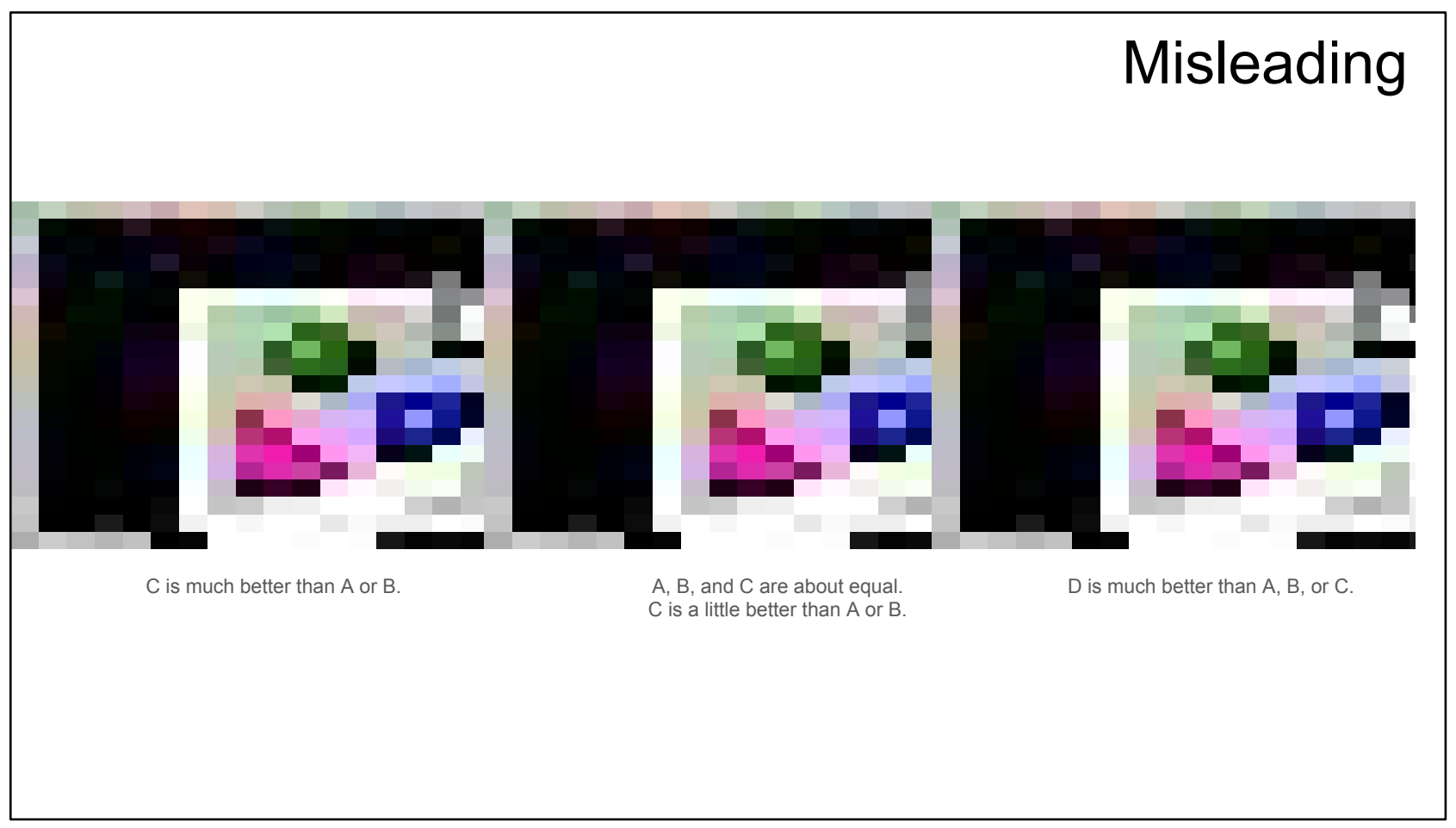

All of these graphs show the same data, in the same bar graph format, but they portray different ideas. Data can be intentionally misleading, or accidentally interpreted. Being shown this data in one way or another could influence our decision-making. 


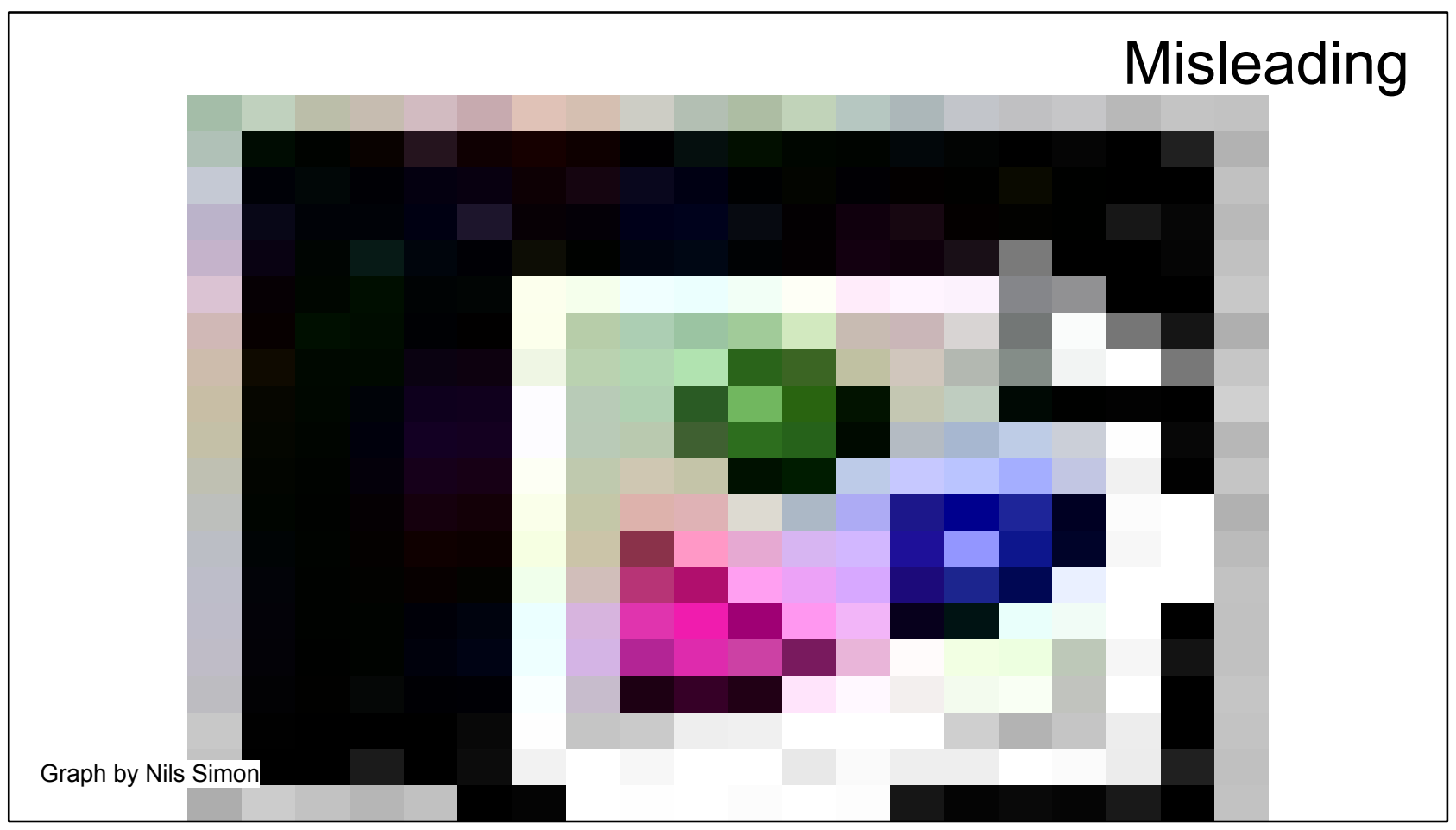

Many graph makers insist "one must always start their y-axis at the zero line", but this is not necessarily ideal. The Keeling Curve is a famous example of this: it shows atmospheric carbon dioxide increasing over time. The context of this data is that a small change in atmospheric carbon can have a huge impact on the climate. This example shows that sometimes it's helpful to truncate the y-axis. If the axis started at zero, the change in slope would appear negligible and the graph would imply it was equally plausible for there to be zero atmospheric carbon dioxide.

Alternative facts are most effective when taken out of context. The importance of preserving context cannot be overstated. 


\section{Avoiding Misinterpretation}

We recently encountered a problem of maintaining context in a 3D visualization. At the end of a sequence about gravitational waves, we were left with an isolated black hole floating in the middle of the galaxy. The client requested that we zoom out to make it appear to be just another distant object in the universe. But because the hole was perfectly symmetrical and the background was infinitely distant, we realized that moving the camera backwards would look identical to shrinking the black hole - which might imply that the black hole was destroying itself.

After some experimentation, we realized that a tilting camera movement that moved the distant background stars across the screen implied that the camera was moving rather than that the black hole was shrinking. In this case, conveying that the changing size of the black hole was part of the context of the camera move was vital to avoiding misinterpretation.

Simulation credit:

LIGO Gravitational Waves: Eliu Huerta, Roland Haas, Gabrielle Allen, University of Illinois at Urbana-Champaign 
Validation 


\section{Validation}

\section{Audience Testing}

Where do these images come from?

A. This is real video, observed through a telescope

B. This is a visualization, based on a scientific computer simulation

C. This is an artistic drawing of what we think it looks like



The AVL works with educational researchers to understand how audiences are interpreting our work. We have held advance screenings of our films to get feedback while there is still time to change the design. 


\section{Validation}

\section{Audience Testing}

$44 \%$ thought it was both real pictures and data visualizations

$33 \%$ thought it was a combination of all three

$23 \%$ thought it was solely visualized data

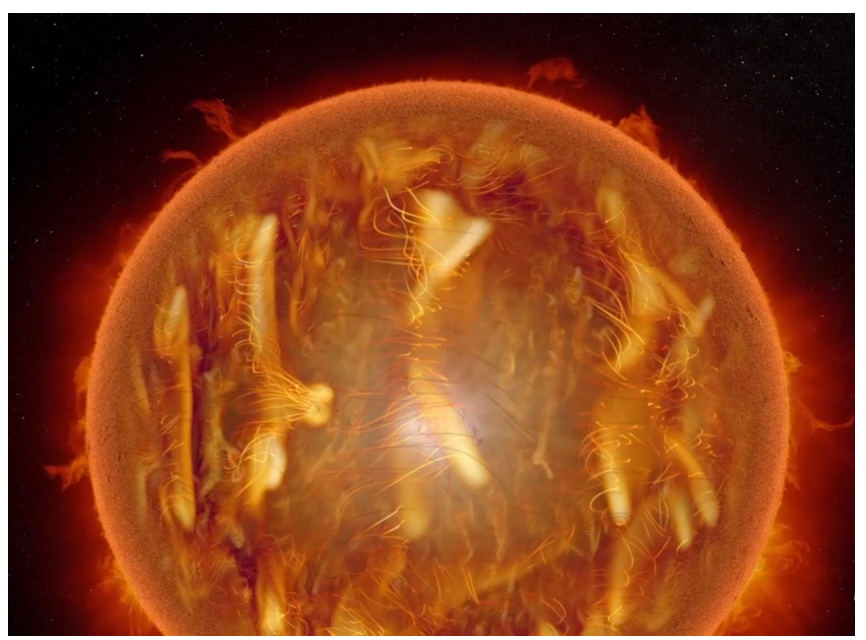




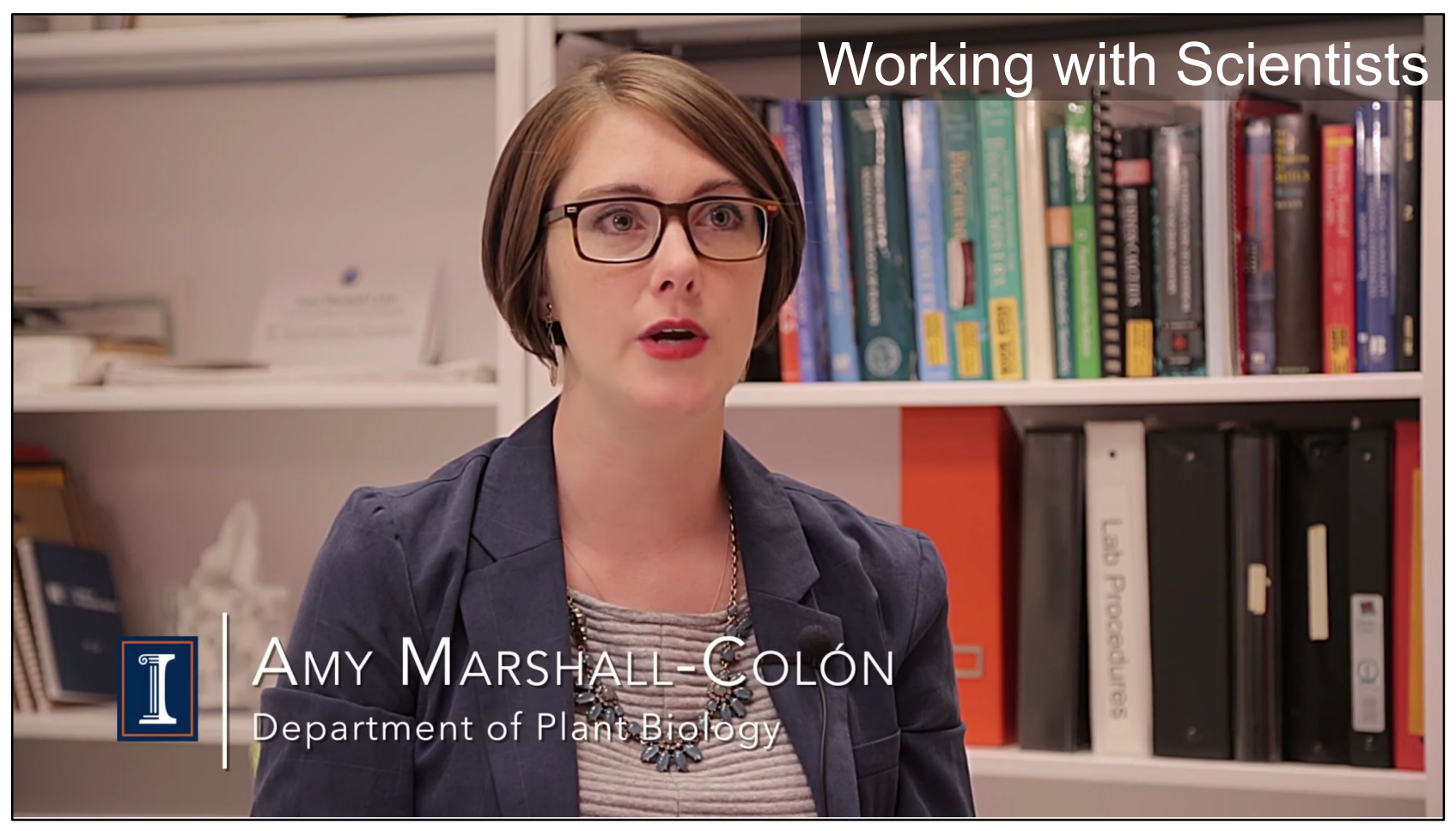

It is also extremely important to have frequent collaborative contact with scientific domain experts so they can identify inaccuracies long before they make it into the film. We work directly with scientists to:

- Understand the purpose of the simulation

- $\quad$ Learn its context in the larger field

- Adapt the documentary script to honestly describe the simulation, rather than force it into a pre-written script

- Determine scientist's ability/willingness to adapt, re-run, or fill gaps in their simulation

- Involve the scientist in iterations on the design of the scene

We also assembled a Science Advisory Committee, which helps us understand and arrange our science narratives from a multidisciplinary perspective. 


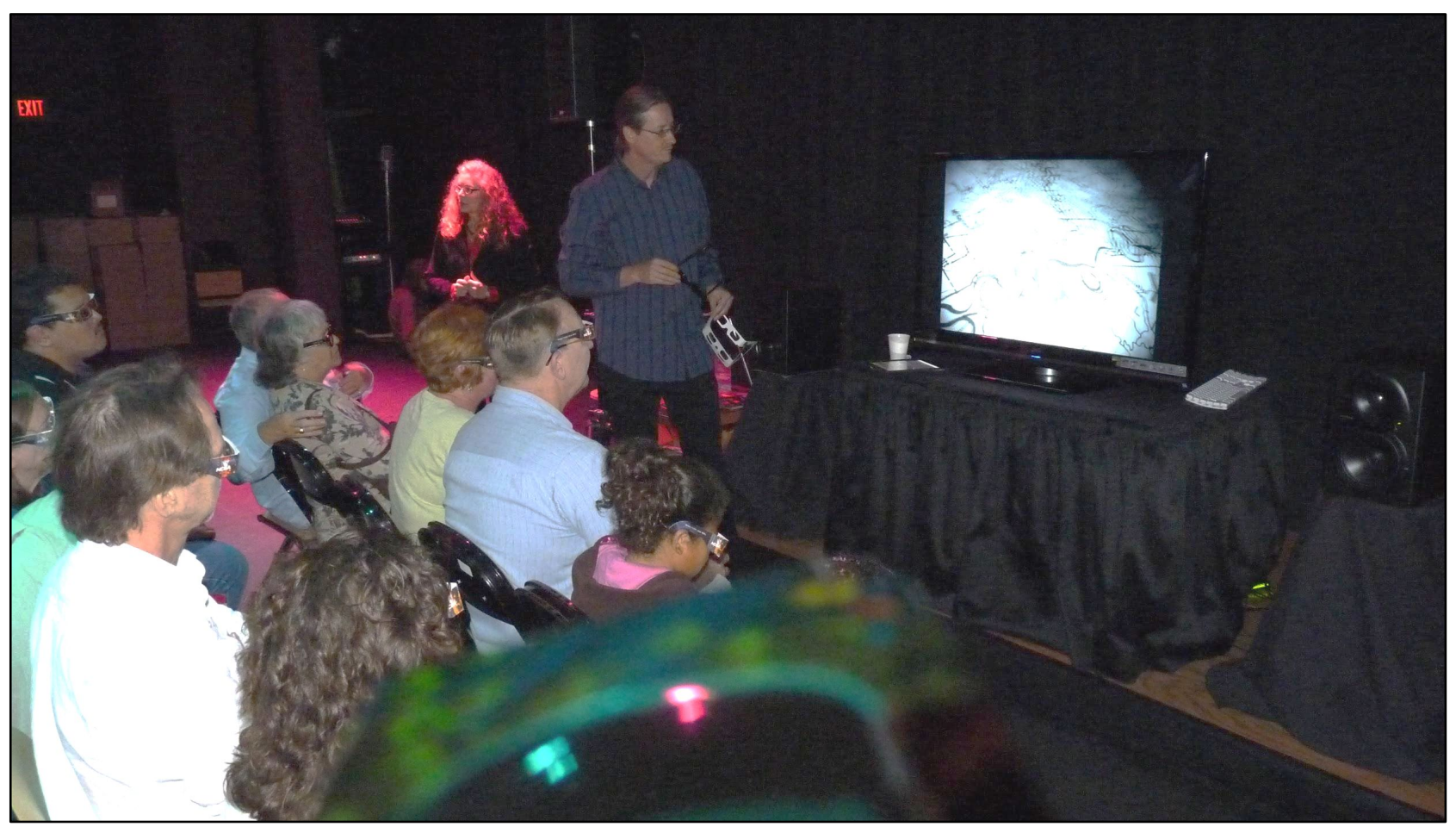

While many design decisions can be influenced by formal research, in most cases there is no formal research to justify making one choice versus another. In these situations, visualization designers must rely on informed intuition.

This is why perhaps the most important way in which we evaluate our work and build our intuition is direct interaction with audiences through daily demonstrations in our lab, and through public presentations and Q\&A sessions like this.

We need to acknowledge that intuition isn't as structured as formal research, and so to avoid falling into the trap of creating our own "alternative facts", we must collect data on these interactions and document and analyze our discoveries periodically. 


\section{Evaluation of Learning Effectiveness}

The National Science Foundation defines the space of cinematic scientific visualization as "informal science", which is measured indirectly. The expectation is not for people to go home and remember facts that they can be tested on (e.g. the sun is 9,941 degrees Fahrenheit), but rather, we want audiences to have an enjoyable experience, to feel empowered, and to be motivated to learn more.

Some of the more important educational outcomes are:

- How many questions have audience members generated by the end of the documentary?

- $\quad$ Are audience members more capable of making scientific explanations to others?

- Do audience members know how to further interrogate the material? 


\section{Black Holes}

\section{The Other Side of Infinity}

(2007)

Narrated by Liam Neelson

\section{Shows have a lifespan of $10+$ years}

\author{
"Black Holes: \\ The Other Side of Infinity" \\ was released in January 2007 \\ and is still in theaters
}

The longevity of our shows is one indicator of successful outreach.

Digital fulldome documentary shows have a lifespan of 10+ years, and IMAX documentaries are not far from that, either. Cinematic scientific visualizations aim to feature the most current cutting-edge science data, but they are applied to timeless questions like "how did we get here?" and feature subjects that have been the focus of research for centuries. 




Our shows are translated into dozens of languages and seen by millions of people, worldwide. "Solar Superstorms" is currently available in Spanish, Mandarin, French, Korean, English, Dutch, Japanese, Czech, German, and Arabic.

There are certain cultural norms associated with the way data can be visualized - for example, in the Western world we may take for granted that green is good and red is bad, but this is not universal. This is why visualizations succeed more often when they reiterate information in multiple ways, and consider the importance of established cross-cultural scientific visual language - for example, representing opposing magnetic forces in red and blue.

The fact that our documentaries cross national and cultural boundaries is another indicator of the success of the way in which our visualizations are packaged. It is important to have a collaborative interaction with international partners to be sure of this success.

Image credits:

Creative commons images courtesy Wikipedia 



Feedback from critics and festivals can be helpful. Although this feedback is not directly aimed at whether audiences absorbed learning objectives, it can address how engaged audiences are with material, it can highlight the strengths and weaknesses of sequences in relationship to each other and in relation to other nominated films, and it can help with evaluation of the overall construction of the documentary. 


\section{/r/EducationalGifs}



Much of our government-funded work is free to download and use for educational purposes, and oftentimes we find our visualizations pop up in unexpected places. Our Global Lambda-Integrated Facilities network map was a backdrop for a scene in the movie "Annie" (2014). A decade-old visualization of ours popped up on the front page of Reddit.com this summer. Our visualizations continue to educate and awe audiences even outside of the contexts for which they have been created.

These discoveries are reinforcement of the impact that these images have on generating questions and contributing to what educational researchers call "agency". "Agency" is the holy grail of educational success: a learner's ability to understand their own questions and their research tools well enough to seek and find answers independently. 


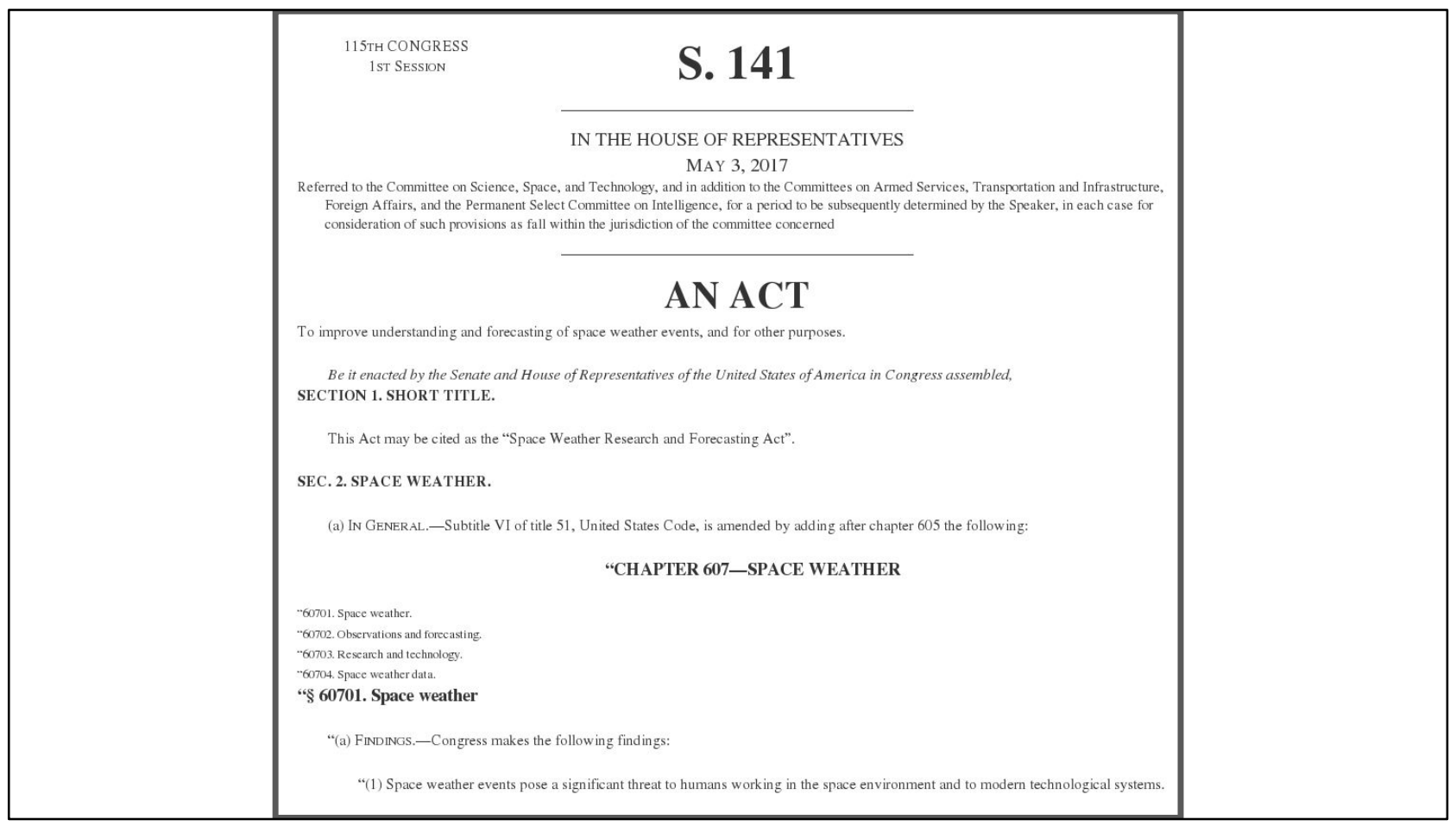

Rarely, visualizations even have very observable impacts on policy makers. In May 2016, AVL Director Donna Cox was invited by the National Science Foundation (NSF) to present "Solar Superstorms" to members of the Senate in Washington, D.C., after the Space Weather Research and Forecasting Act was introduced. In May 2017, the Act passed the Senate unanimously.

While we do keep in mind that that the target audience for our visualizations is the "general public", it is important to remember that experts and policy makers are among that public. This is why it is crucially important for our representations and explanations of science to be not only visually appealing, but unambiguous and truthful. 


\section{Re-Use for maximum Impact}

It can take a significant amount of effort to collect scientific data and build a visualization pipeline that can handle it, which seems hard to justify for the purposes of a two-minute video sequence, especially considering that audiences are likely leaving their first exposure to the data with the most basic understanding of it. 


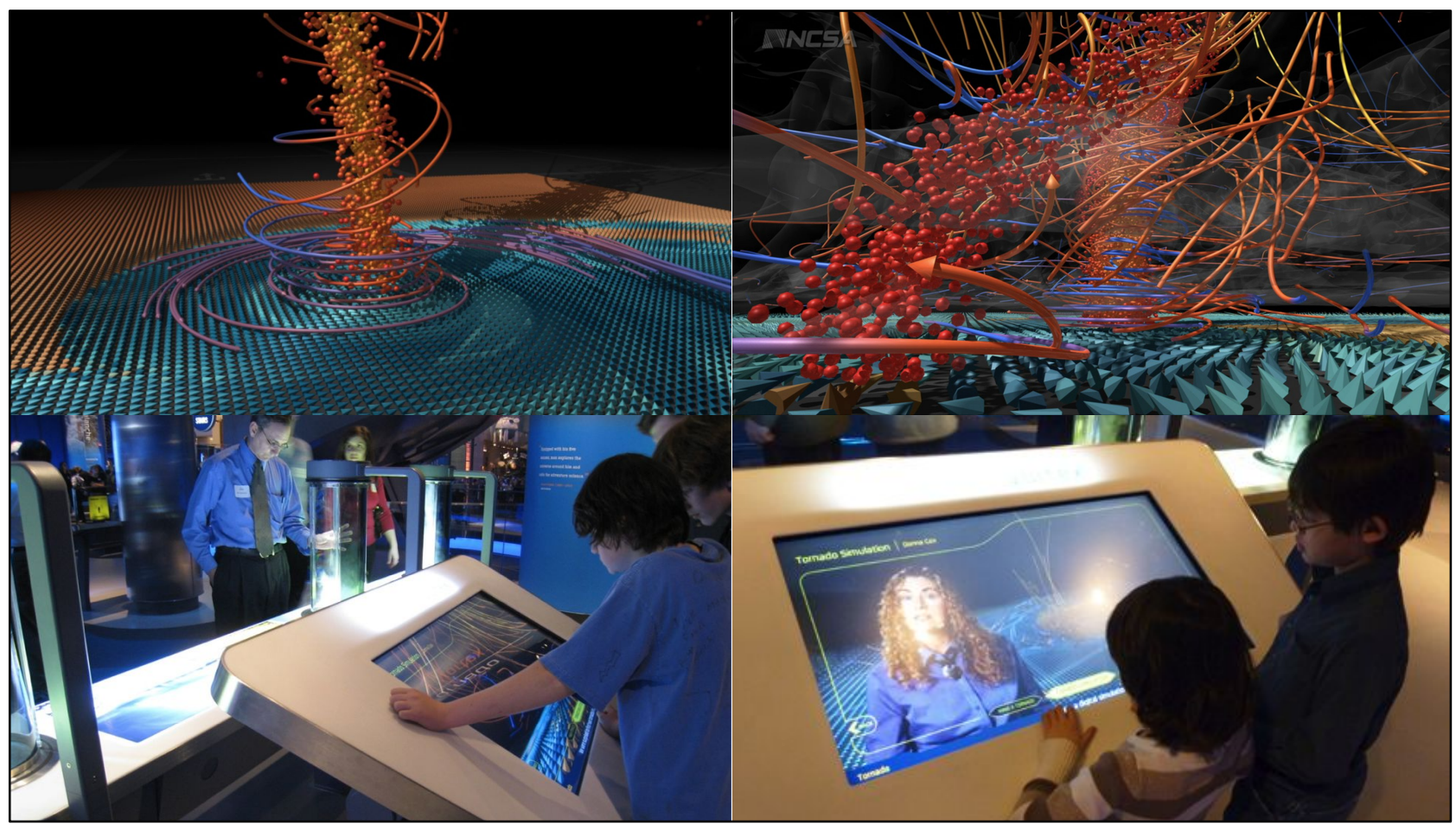

Educational literature suggests that re-contextualizing data is of enormous value for learners. Placing a data set in multiple appropriate contexts or choosing different representations can help people expand their mental models. Furthermore, an inspirational cinematic experience can be complemented by an interactive interrogation, for instance.

The AVL does not dispose of simulation data after a single use - rather it gets saved along with its pipeline, and reused in different contexts for different types of distribution, from films, to television, to live arts performances, to museum kiosks, to interactive laboratory demonstrations. 




Our professional education collaborators also create supplemental educational materials to go along with our documentaries, such as the booklet pictured here. This repackaging of the information in a more factually intensive resource students can refer to after they've seen the film to answer their more in-depth questions. 


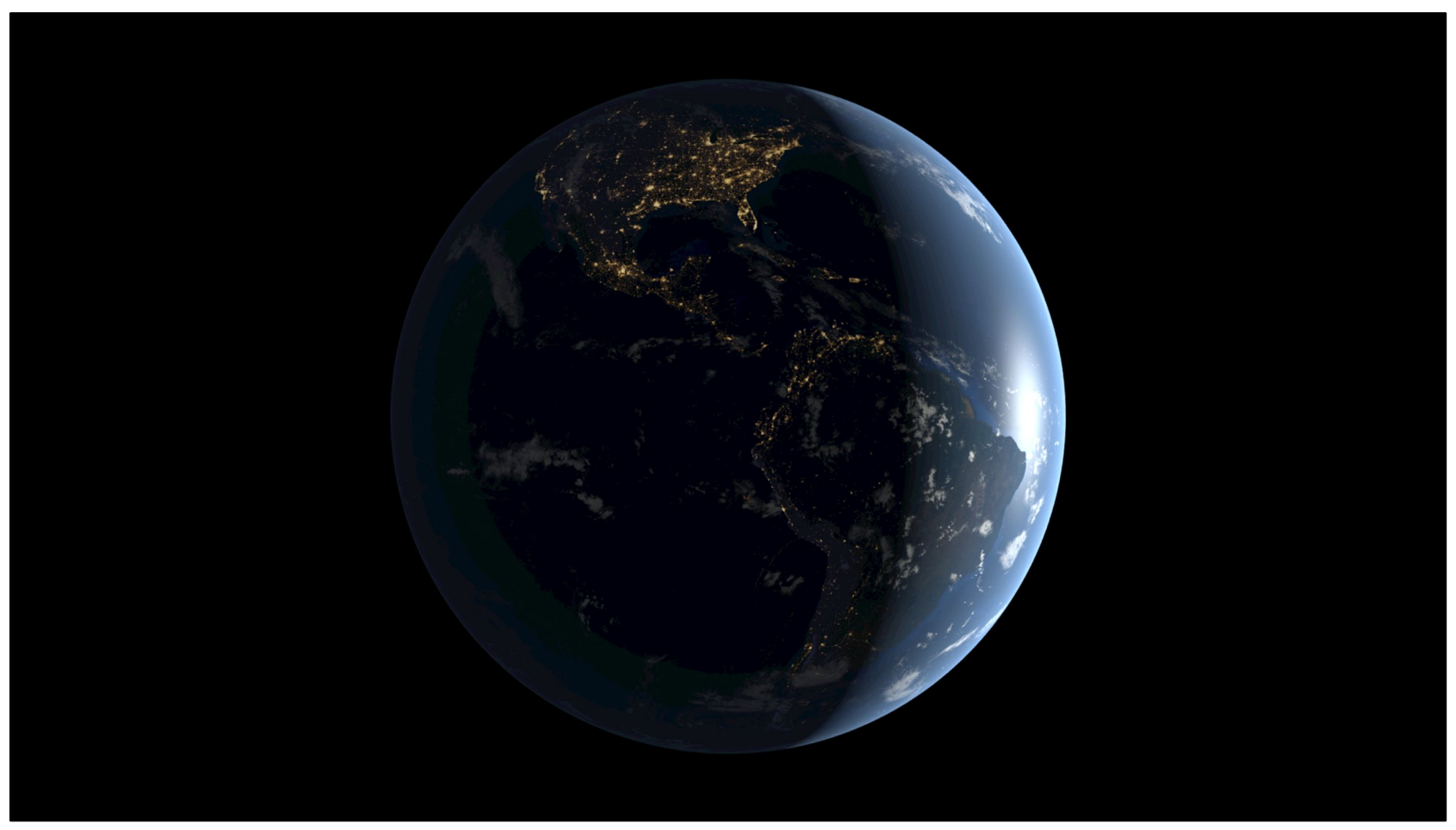

The field of visualization is only growing, and by combining all these techniques, cinematic scientific visualization is becoming a significant contributor to the scientific literacy of people around the world.

Image credit:

Blue Marble image texture courtesy NASA 
Thank You 


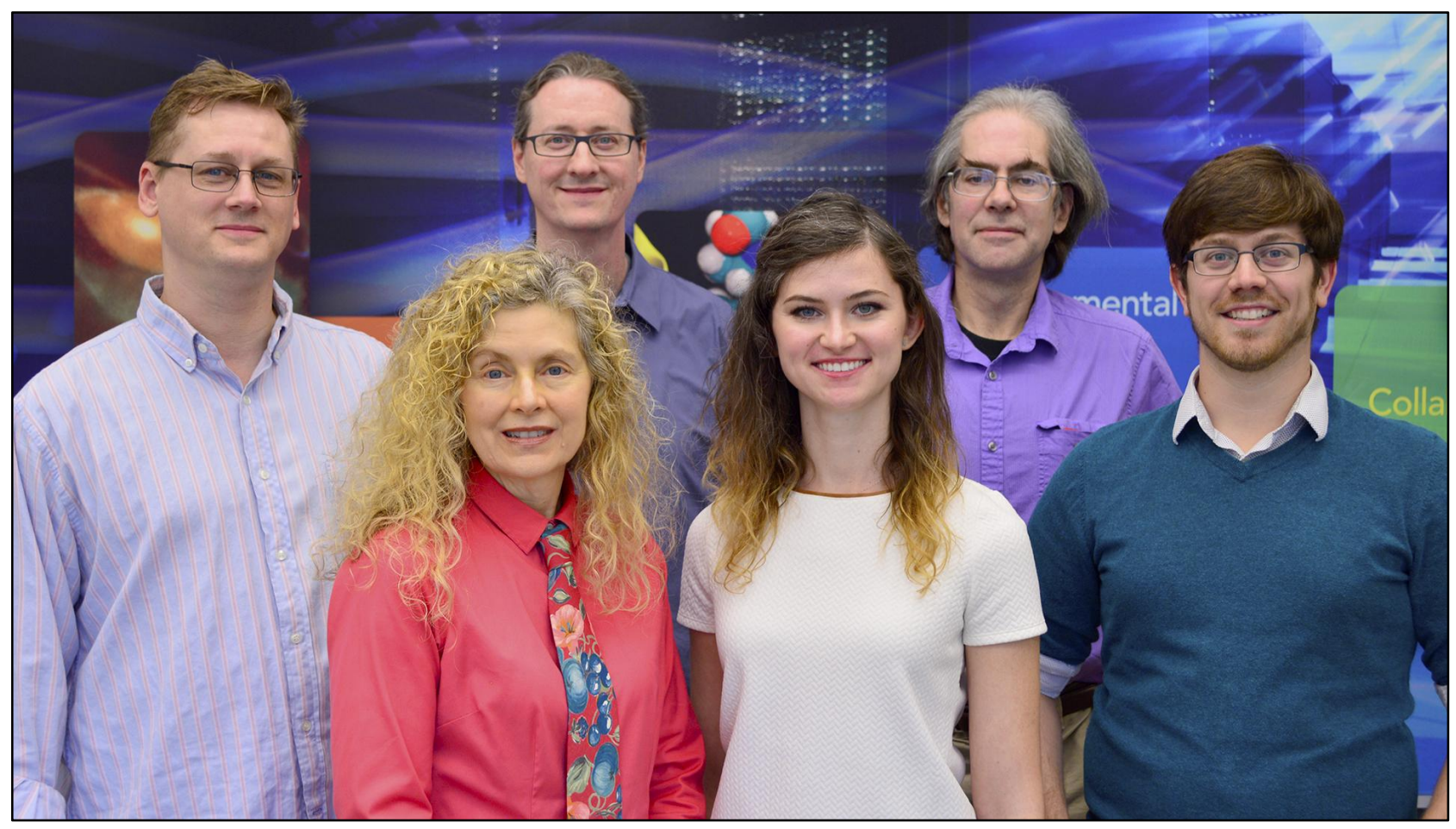

The Advanced Visualization Lab: Jeff Carpenter, Bob Patterson, Stuart Levy

Donna Cox (director), Kalina Borkiewicz, AJ Christensen 


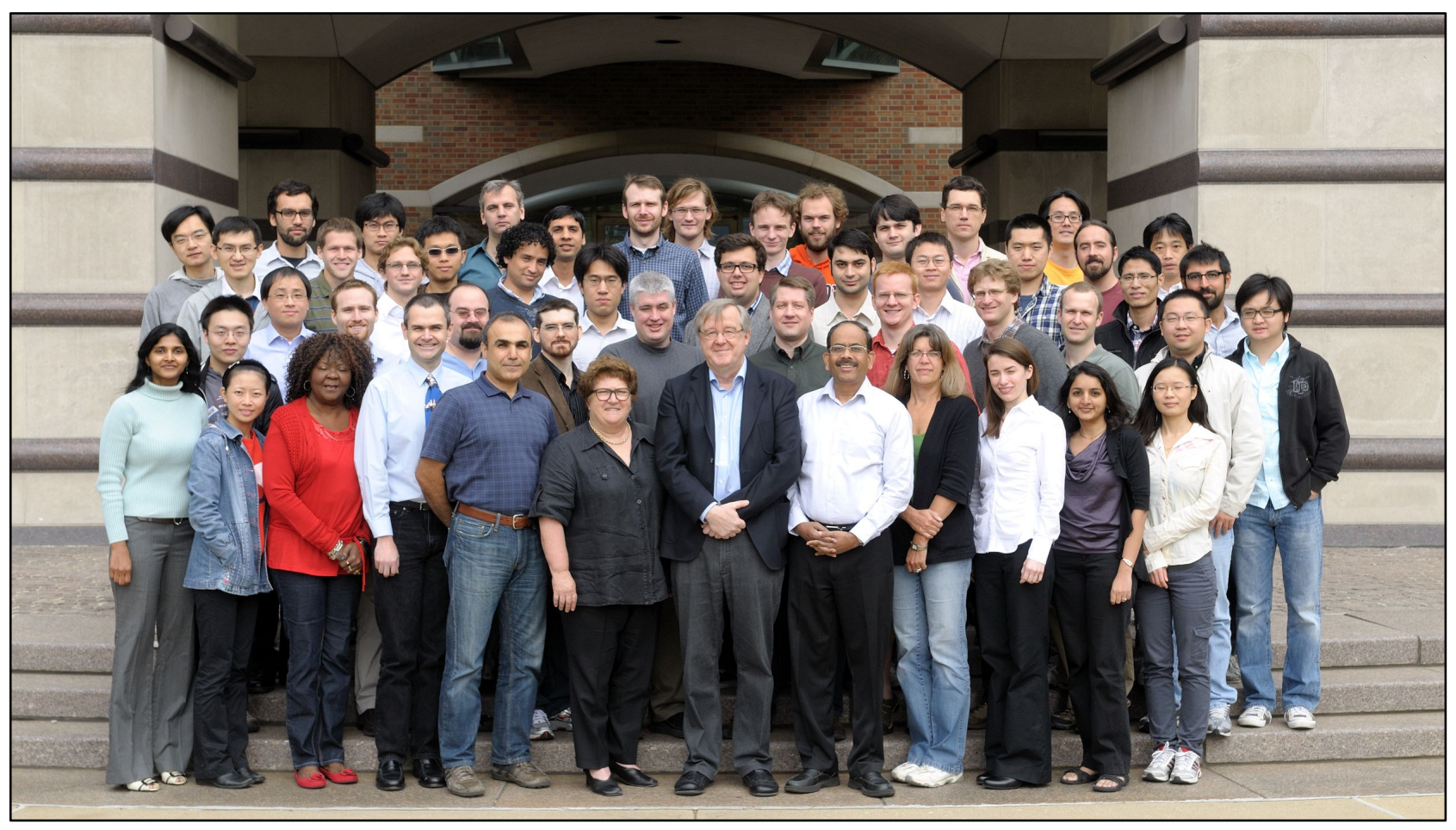

The Theoretical and Computational Biophysics Group 


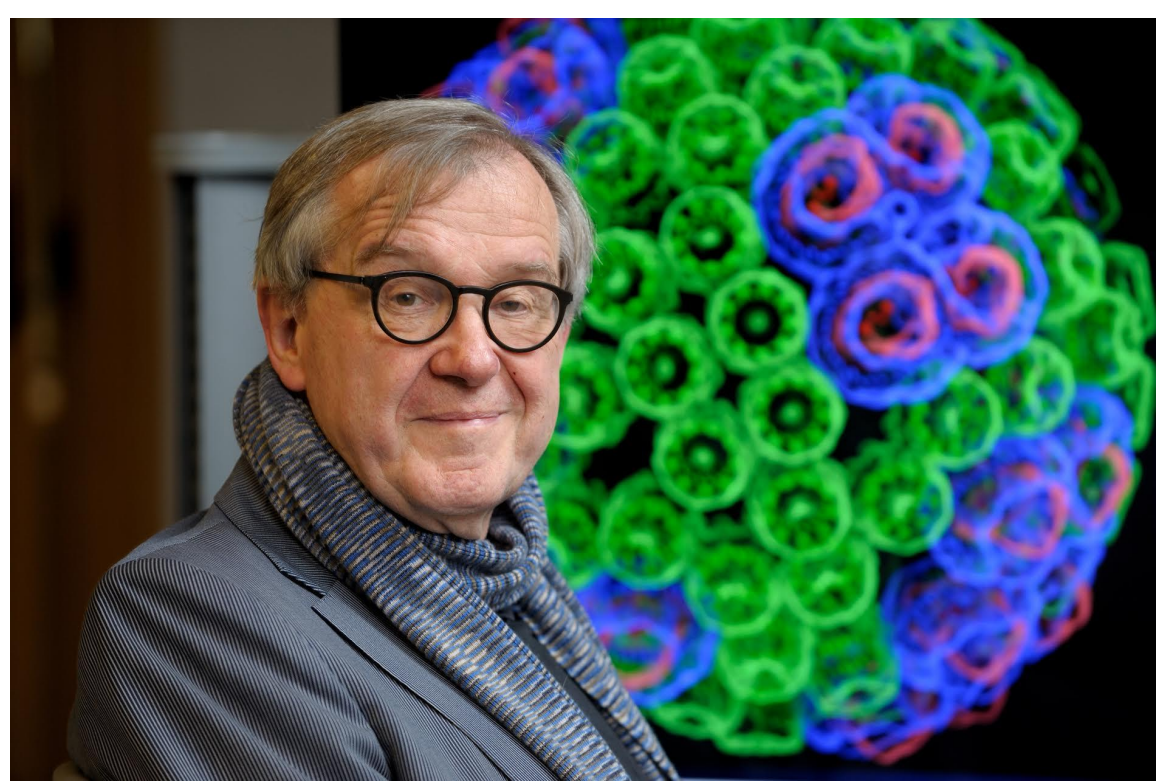

"When I was a young man, my goal was to look with mathematical and computational means at the inside of cells, one atom at a time, to decipher how living systems work. That is what I strived for and I never deflected from this goal." - Klaus Schulten 